The Ecology of Organizational Forms in Local and Regional Food Systems: Exploring the Scaling-Up Challenge via a Species Concept

\author{
By \\ Jason Scott Entsminger
}

\author{
A Dissertation presented to \\ the Faculty of the Graduate School \\ at the University of Missouri - Columbia
}

In partial fulfillment of the requirements for the degree

Doctor of Philosophy

DECEMBER 2020 


\section{(C) 2020 Jason Scott Entsminger}

All rights reserved. 
Those listed, appointed by the dean of the Graduate School, have examined the dissertation entitled

\section{The Ecology of Organizational Forms in Local and Regional Food Systems: Exploring the Scaling-up Challenge via a Species Concept}

presented by Jason Scott Entsminger,

a candidate for the degree of Doctor of Philosophy

in the field of Agricultural and Applied Economics,

and hereby certify that, in their opinion, it is worthy of acceptance.

Professor Randall Westgren

Professor Harvey James

Professor Sarah Low

Professor André Ariew

Professor Peter Foreman 
To my grandfather, Lewis Sterlacci, a master carpenter, union leader, chess player, and the man who showed me that the pursuit of intellect is a passion for all those who seek knowledge and wisdom. 


\section{ACKNOWLEDGMENTS}

The research presented in this work was supported in part by funds from the National Institute of Food and Agriculture (NIFA; award MICL08493) and endowments at University of Missouri generously made by the McQuinn Family. The views and analysis expressed herein are solely those of the author and do not represent an official position of the US government or its agencies, including NIFA. The author thanks his advisor, Dr. Randall Westgren, for years of support, intellectual development, and collegiality. Similar thanks go to the members of his committee, listed above, and to Dr. Joseph Parcell, formerly of the committee, for their advice and encouragement. Throughout this course of this project, numerous colleagues from many institutions across the globe have provided feedback, alternative perspectives, and praise during conferences, working paper sessions, and seminars; they too are thanked for their contributions to this project and its author's academic development. 


\section{TABLE OF CONTENTS}

\section{page}

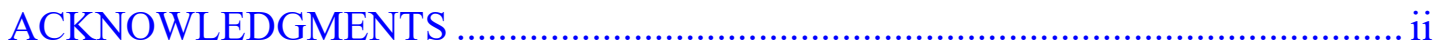

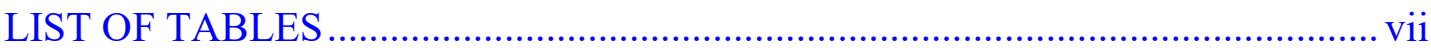

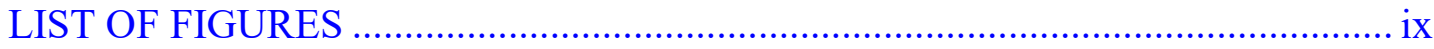

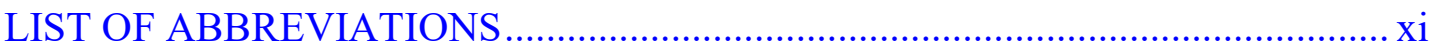

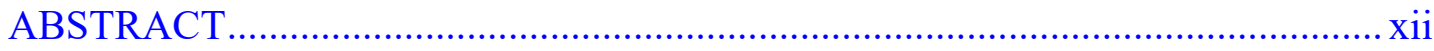

\section{CHAPTER}

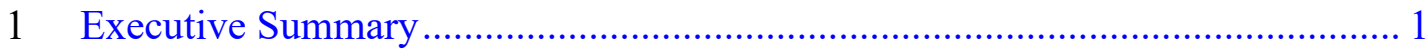

1.1 Local and Regional Food Systems: Growing Prominence Without Clear

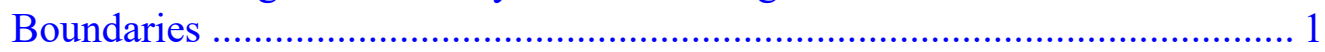

1.2 A Framework for Understanding AFSs .................................................. 2

1.3 Applied Science: Understanding the Ecology of LRFSs to Address the Scaling-Up Challenge ............................................................................. 3

1.4 Fundamental Science: Addressing the Classification Problem in the Theory of Organizational Evolution.................................................................... 5

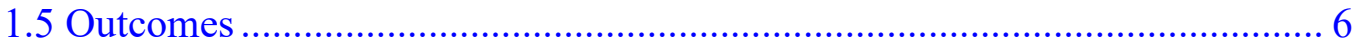

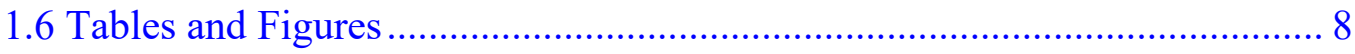

2 Local and Regional Food Systems and the Scaling-up Challenge ..................... 9

2.1 Building and Applying a Framework to LRFSs ..................................... 10

2.1.1 Understanding AFSs at Three Levels: Social Movements, Economic Arrangements, and Agents.............................................................. 11

2.1.1.1 Social Movements as the Catalyst for AFSs ......................... 11

2.1.1.2 Systems as Networks of Agents ........................................... 17

2.1.2 Proposing a Multilevel Model for Greater Consistency and Clarity .. 18

2.1.2.1 Embeddedness and Alternative Arrangements of Exchange ... 20

2.1.2.2 Adopting a Systems Definition of the Meso Level ................. 23

2.2 Local and Regional Foods: Achieving Alterity through a "Commitment to Place" 25

2.2.1 Establishing a Distinct Macro Level .............................................. 26

2.2.2 Delineating the Imaginary of the Local and Regional Foods

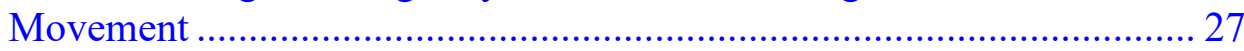

2.2.2.1 "Commitment to Place" as a Unificatory Imaginary ............... 30

2.2.2.2 Dimensions of Proximity: Operationalizing "Commitment to Place" to Define LRFSs ............................................................... 32

2.2.3 Understanding the Meso Level: SFSCs as the Substance of LRFSs.. 34

2.2.4 The Value of the Conceptual Framework ...................................... 37 


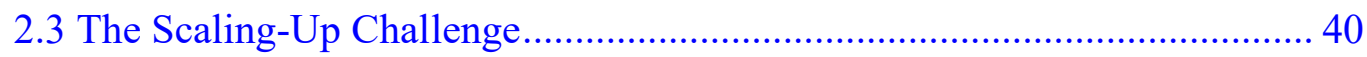

2.3.1 Defining the Scaling-Up Challenge.................................................... 40

2.3.2 Incorporating Arrangements That Can Scale Up LRFSs: Why

Integration With the SFSCs Concept Matters............................................. 45

2.3.3 The Meso- and the Micro-: Taking an Ecological Approach to Understand Agents and Networks That Achieve Scale in LRFSs ........... 48

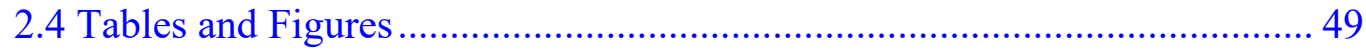

3 Ecologies and Organizational Forms: Developing an Organizational Species

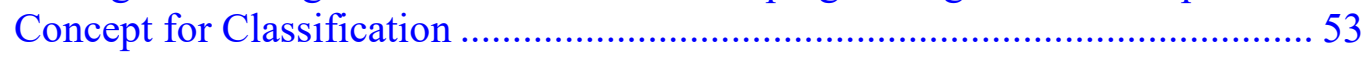

3.1 Ecological Allegory in the Social Sciences: Understanding Econosystems 54

3.1.1 What Is Ecology? What Are Ecosystems? ………………………….... 55

3.1.2 The Role of Kinds in Ecologies: The Need to Classify.......................5 57

3.1.3 Understanding Networks of Agents Using Ecological Allegory ........ 60

3.2 A Philosophical Note: The Social Ontology of Organizations ......................... 61

3.2.1 The Firm: Economic Perspectives on the Organization and

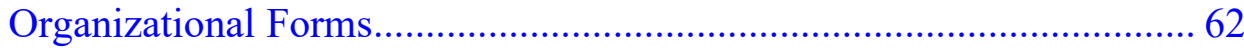

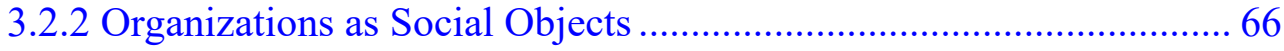

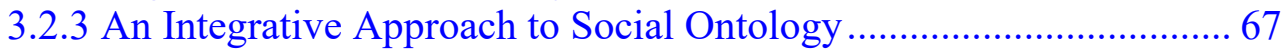

3.3 Organizational Systematics: Classification in the Social Sciences ............... 70

3.3.1 Fundamentals of Classification Theory in the Social Sciences.......... 72

3.3.2 Organizational Systematics: A Taxonomic Approach to Classification

3.4 Developing a Framework for Organizational Classification to Delimit Forms

3.4.1 Patterns and Properties: Integrating Boyd, Searle, and Epstein ......... 85

3.4.2 Econological Niches: Adaptations of the Niche Construct in Economics, Management, and Organizational Sciences ......................... 90

3.4.2.1. Ecology: The Environmental Niche and the Population Niche

3.4 .22 Niche Construction

3.4.2.3. Bridging the Metaphorical Divide........................................... 94

3.4.3 The Organizational Species Concept: At the Nexus of Kinds and

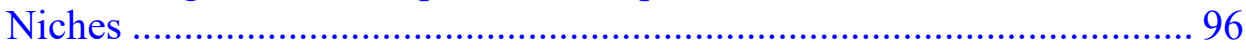

3.5 Operationalizing the Organizational Species Concept: A Methodology to

Search for HPCs and Identify and Validate Kinds............................................. 97

3.5.1 Adopting a Hierarchical Clustering Analysis Approach .................... 100

3.5.2 Procedures for Identifying Kinds and Validating Them as Species. 102

3.5.2.1 Selection and Refinement of Variable Sets ............................ 103

3.5.2.2 Formation of a Final Postulated Cluster Solution ................... 107

3.5.2.3 Acceptance or Rejection of FPCS as a Set of Organizational

Species ................................................................................... 112

3.5.3 Adopted Methodology, Abductive Reasoning, and Inferences........ 114

3.6 Concluding Remarks: The OSC, Species, Ecologies, and Scale …….......... 117

3.7 Tables and Figures ...................................................................................... 119 
4 Applying the Organizational Species Concept: Classifying Food Hub

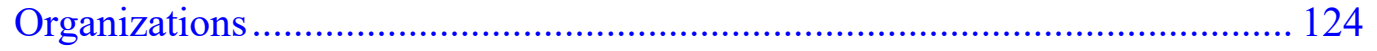

4.1 A Primer on Food Hub Organizations ....................................................... 125

4.1.1 The Basic Concept of the Food Hub Organization .......................... 127

4.1.2 Economic Organizations Rooted in a Social Movement................... 136

4.1.3 Hybrid Organizations: Economic Theories of Existence ................... 141

4.1.4 Multiple, Heterogeneous Stakeholders............................................. 147

4.1.5 Putting It All Together: How the Theory of FHOs Drives the Search

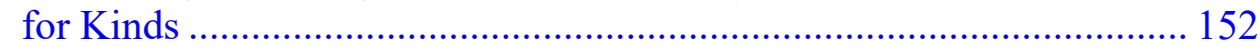

4.2 Data: The 2015 National Food Hub Survey ................................................ 154

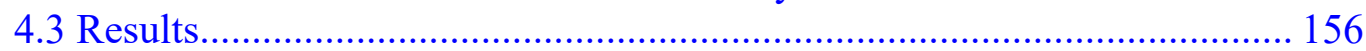

4.3.1 Initial Variable Selection: Theoretical and Empirical Motivations.. 157

4.3.2 Preliminary Clustering Models: Results From Iterative Rounds Exploring Variable Sets and Parameters................................................ 160

4.3.3 Final Postulated Cluster Solution ...................................................... 163

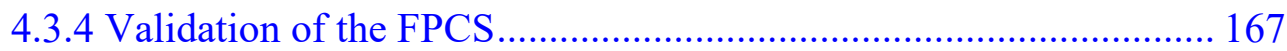

4.3.4.1 ANOVA and Independent-Samples $t$ Test Results: Differences in Variances and Means................................................................. 168

4.3.4.2 Regression Analysis Results .............................................. 172

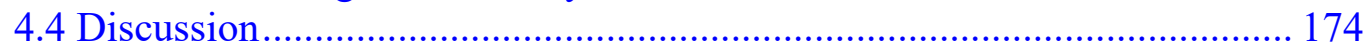

4.5 Concluding Remarks ......................................................................... 176

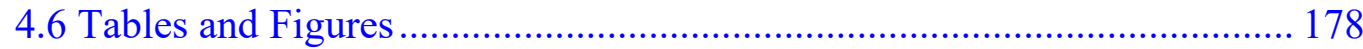

5 The Ecology of Food Hub Organizations: Implications for Policy on Scaling up Local and Regional Food Systems ……………………………………….... 214

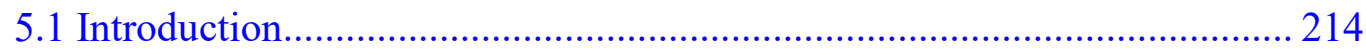

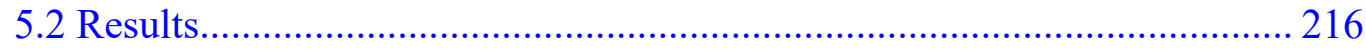

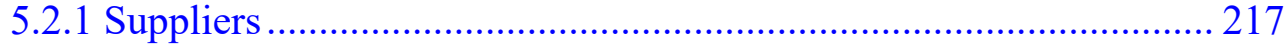

5.2.2 Managerial and Financial ............................................................... 221

5.2.3 Mission and Daily Operations ......................................................... 226

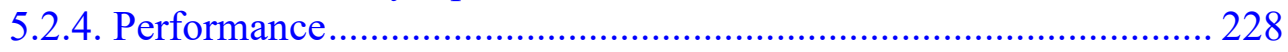

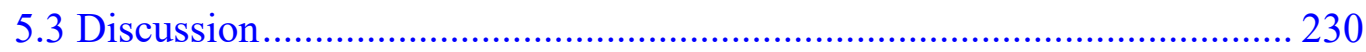

5.4 Policy Implications and Conclusions......................................................... 233

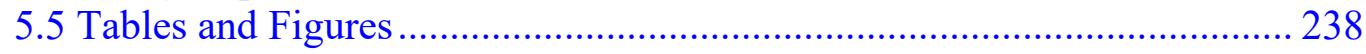

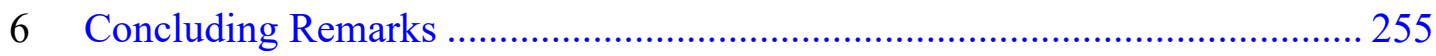

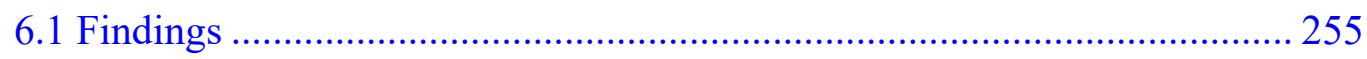

6.1.1 Outcomes of Classification............................................................ 256

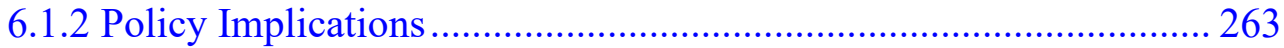

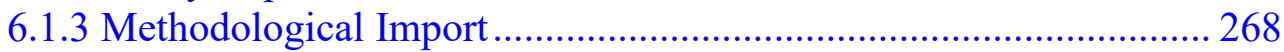

6.2 Limitations Encountered in this Study .................................................. 272

6.2.1 Data Quality and Completeness …………………......................... 272

6.2.2 Methods and Stage of Research .................................................... 274

6.3 Extensions and Future Research ................................................................ 276 
6.3.1 Extensions With FHOs and Other Organizational Populations in

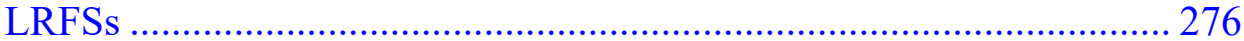

6.3.2 Enabling an Ecological Approach: Use of the OSC for Modeling Econosystems and Implications for Organizational Evolution Theory . 278

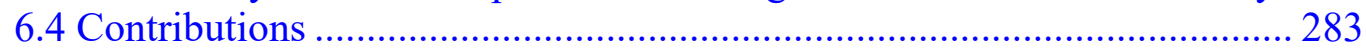

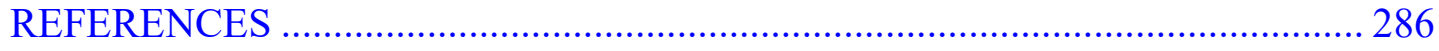

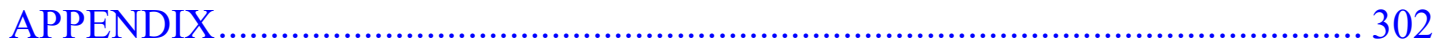

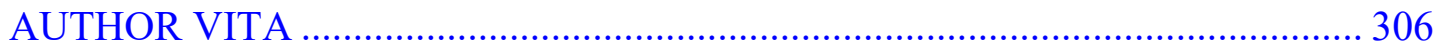




\section{LIST OF TABLES}

$\underline{\text { Table }}$

page

2.1 Comparative Summary of Paradigm Dimensions: Conventional versus

Alternative Agriculture

2.2 Summary of SFSC Types.............................................................................. 52

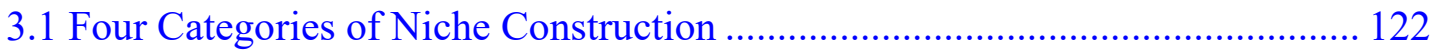

4.1. Summary of Variable Sets in Interative Cluster Analysis Rounds .................... 182

4.2. Summary of Additional Variables Considered for Use in Applying the Organizational Species Concept to Food Hub Organizations......................... 183

4.3. Selected Results of the Correlation Analysis: Morphological Variable Comparisons Only

4.4. Selected Results of the Correlation Analysis: Morphological Variables

Compared With Outcome Variables.

4.5. Selected Results of the Correlation Analysis: Outcome Variable Comparisons

Only.....

4.6. Statistical Indexes of Model Performance for Variable Sets E and F.

4.7. Variables With Statistically Significant Mean Differences Between In-Sample and Out-of-Sample Cases

4.8. Matrix of Adjusted Rand Index Values for Selected Models and Partitioning Solutions

4.9. Normalized Raw Stress Values of the Final Variable Sets for 2 Through 4 Dimensions

4.10. Node Heights and Differences for Model 5

4.11. Summary of Statistical Significance of Multigroup Analysis of Variance for Selected Partitioning Solutions of Model 5: Morphological Variables ........ 196

4.12. Summary of Species Populations and Their Niche, as Represented by Strategic Orientations.....

4.13 Summary of Statistical Significance of Multigroup Analysis of Variance for Selected Partitioning Solutions of Model 5: Niche Variables for Channel and Product Reliance 204 
4.14 Summary of Statistical Significance of Multigroup Analysis of Variance for Selected Partitioning solutions of Model 5: Niche Variables for Community Service Offerings 205

4.15 Mean Differences in Species Morphological Variables: Each Species Compared With All Other Observations ......................................................................... 206

4.16 Mean Differences in Species Niche Variables: Each Species Compared With All Other Observations ............................................................................ 207

4.17 Mean Differences in Species Product and Channel Reliance Variables: Speciesto-Species Comparisons ............................................................................... 208

4.18. Mean Differences in Species Community Service Offerings Variables: Speciesto-Species Comparisons........................................................................... 209

4.19. Mean Differences in Species' Morphological Variables: Species-to-Species Comparisons 210

4.20 Regression Analysis Results for Validation Stage: Channel Reliance and Community Services

4.21 Regression Analysis Results for Validation Stage: Product Reliance in Primary Product Categories

4.22 Regression Analysis Results for Validation Stage: Product Reliance in Secondary Product Categories

5.1 Mean Differences in Supplier and Manager Experience Variables: Species Versus All Other Observations 238

5.2 Mean Differences in Financial and Performance Variables: Species Versus All Other Observations

5.3 Mean Differences in Relevance of Social Values to Mission and Day-to-Day Operations Variables, With Each Species Compared With All Other Observations 253

5.4 Regression Analysis Results for FHO Performance Variables............................ 254

A.1 Numerical Data for Species Mean Profiles: Morphological Attributes............ 302

A.2 Numerical Data for Species Mean Profiles: Product Reliance ……………....... 303

A.3 Numerical Data For Species Mean Profiles: Channel Reliance ........................ 304

A.4 Numerical Data for Species Mean Profiles: Community Services .................... 305 


\section{LIST OF FIGURES}

$\underline{\text { Figure }}$

page

1.1 Total number of academic publications per year identified under three search

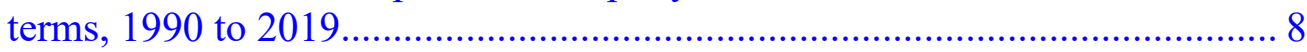

2.1 Multilevel Relational Model of Alternative Food Mechanisms ......................... 50

2.2 Flow Chart of Alternative Foods Space .......................................................... 51

3.1 Conceptual Framework of the Social Ontology of Organizational Forms ........ 119

3.2 The Ecological Niche in Reduced-Dimension Resource Space ...................... 120

3.3 Overlapping Fundamental Niches in the Ecological Hyperspace..................... 121

3.4 History of the Niche Concept in Biology and Sociology ............................... 123

4.1. Defining Characteristics of a Regional Food Hub ..................................... 178

4.2 Common Conceptual Model of Sustainability............................................. 179

4.3 Legal Structures of Food Hub Organizations ............................................. 180

4.4 Percentage of Food Hub Organizations Procuring Products From Different

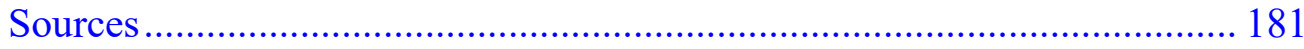

4.5. Representation of Cases in Three-Dimensional Space: Model 1, $k=11 \ldots \ldots \ldots 191$

4.6. Representation of Cases in Three-Dimensional Space: Model 2, $k=12 \ldots \ldots \ldots 192$

4.7. Representation of Cases in Three-Dimensional Space: Model 5, $k=9 \ldots \ldots \ldots \ldots 193$

4.8. Representation of Cases in Three-Dimensional Space: Model 6, $k=3 \ldots \ldots \ldots \ldots 194$

4.9. Error Bar Chart: Variables Representing Organization Morphology …........... 197

4.10. Dendrogram of Clustering Solutions Under Model 5 With the Six Partitions of the Final Postulated Solution and Species Names Indicated $(k=6)$.......... 200

4.11. Representation of Cases in Three-Dimensional Space: Model 5, $k=6 \ldots \ldots \ldots . .201$

4.12. Error Bar Chart: Variables Representing Channel and Product Reliance ...... 202

4.13. Error Bar Chart: Variables Representing Community Services ................... 203

5.1 Mean Profiles of FHO Species: Types of Suppliers Used .............................. 240 
5.2 Types of Suppliers Used, by FHO Species........................................................ 241

5.3 Mean Profiles of FHO Species: Demographics of Suppliers.............................. 242

5.4 Demographics of Suppliers, by FHO Species................................................... 243

5.5 Mean Profiles of FHO Species: Experience of Top Manager in Key Functions of

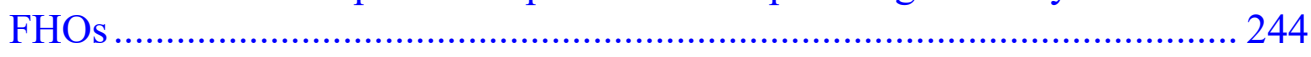

5.6 Manager Experience in Key Functions, by FHO Species.................................. 245

5.7 Mean Profiles of FHO Species: Sources of Startup Funding ............................. 246

5.8 Sources of Startup Funding, by FHO Species ………………………………. 247

5.9 Mean Profiles of FHO Species: Percentage of Total Costs for Each Category. 248

5.10 Percentage of Total Costs for Each Category, by FHO Species...................... 249

5.11 Mean Profiles of FHO Species: Relevance of Social Values to Organization Mission and Day-to-Day Operations ......................................................... 250

5.12 Relevance of Social Values to Organization Mission, by FHO Species ......... 251

5.13 Relevance of Social Values to Organizations' Day-to-Day Operations, by FHO

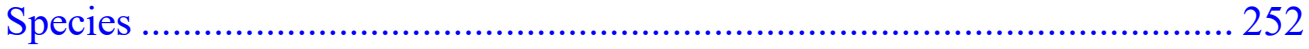




\section{LIST OF ABBREVIATIONS}

$\begin{array}{ll}\text { AFN } & \text { alternative food network } \\ \text { AFS } & \text { alternative food system } \\ \text { ANOVA } & \text { analysis of variance } \\ \text { AOTM } & \text { agriculture of the middle } \\ \text { ARI } & \text { Adjusted Rand index } \\ \text { CCC } & \text { cophenetic correlation coefficient } \\ \text { CES } & \text { collective entrepreneurial strategies } \\ \text { CHI } & \text { Calinski-Harabasz index } \\ \text { CI } & \text { confidence interval } \\ \text { CSP } & \text { Community Services Providers (a species of food hub organizations) } \\ \text { DBSCAN } & \begin{array}{l}\text { denisty-based spatial clustering of applications with noise (a type of } \\ \text { data clustering algorithm) }\end{array} \\ \text { DTC } & \text { direct-to-consumer (marketing channels) } \\ \text { DTI } & \text { direct-to-institution (marketing channels) } \\ \text { DTM } & \text { direct-to-intermediary (marketing channels) } \\ \text { DTR } & \text { direct-to-retail-and-restaurant (marketing channels) } \\ \text { FHO } & \text { food hub organization } \\ \text { FPCS } & \text { final postulated clustering solution } \\ \text { HCA } & \text { hierarchical cluster analysis } \\ \text { HPC } & \text { homeostatic property cluster } \\ \text { LFN } & \text { local food network } \\ \text { LRFM } & \text { local and regional food movement } \\ \text { LRFS } & \text { local and regional food system } \\ \text { MDS } & \text { multidimensional scaling } \\ \text { OSC } & \begin{array}{l}\text { organizational species concept } \\ \text { short food supply chain }\end{array} \\ \text { SSS } & \text { Small-scale Startups (a species of food hub organizations) } \\ \text { TPW } & \text { Traditional Producer Warehousers (a species of food hub } \\ & \text { organizations) } \\ & \end{array}$




\title{
ABSTRACT \\ The Ecology of Organizational Forms in Local and Regional Food Systems: Exploring the Scaling-up Challenge via a Species Concept
}

\author{
Jason Scott Entsminger
}

Dr. Randall E. Westgren, Dissertation Supervisor

Over the past 30 years, Western nations have developed alternative systems for exchanging agrofood products which incorporate social values into the transactional environment. These systems are comprised of many different exchange relationships, structured to transmit information about social values and attach credence attributes to the products. New organizational forms, institutions, and networks arise to achieve the values demanded by the underlying social movement. One movement centers on the social value of a commitment to place. It seeks to create relocalized and socially embedded means of exchange. Policy initiatives have responded, making investments in local and regional food systems. The primary challenge faced by these initiatives: how to increase the of scale while maintaining the value premiums associated with the movement's objectives.

I view these complex networks as ecologies and seek to understand how different organizational forms interact to scale-up LRFSs. I make three crucial developments: (1) a framework to define LRFSs; (2) a model on the metaphysics of social objects and their kinds; and (3) an Organizational Species Concept to consistently identify organizational forms. Together these developments enable an ecological approach by providing a means of identifying distinct organizational populations. I apply my OSC to the case of food hubs - coordinating intermediaries identified as a key for increased scale. This yields six "species". I find that each fills a different functional role and contributes differently to scaling-up LRFSs. I highlight how this is helpful for targeted policymaking. 


\section{CHAPTER 1 \\ EXECUTIVE SUMMARY}

\subsection{Local and Regional Food Systems: Growing Prominence Without Clear Boundaries}

Over the past 30 years, Western nations have made substantial investments to develop alternative systems for exchanging agrofood products so that specific social values are incorporated into the transactional environment. These systems are comprised by a variety of exchange relationships whose structures enable the transmission of information about the social values at play, and thus which attach credence attributes to the products and services being sold. As alternatives to the conventional means which became common during the industrialization of the agrofood sector, these systems include transactional arrangements that produce, transform, and distribute agrofood products in ways that counter the values - or lack thereof - perceived to exist in the conventional systems for accomplishing these tasks. These alternative arrangements include new organizational forms, new institutions, and new networks of economic agents.

Different terms, concepts, and goals abound, including so-called local and regional food systems (LRFSs), whose attention has grown in North America and particularly in the United States. An example of this is shown in Figure 1.1, which reports the number of academic publications between 1990 and 2019 with the keywords "local foods," "alternative food systems" (AFSs) and "local and regional food systems" (LRFSs) (Clarivate Analytics, 2020). It is also seen in the legislative history of US farm policy, with the most recent Farm Bill including a number of earmarked programs and the creation of dedicated agencies at the US Department of Agriculture specific to source-identified production and marketing channels. The US Department of 
Agriculture's Economic Research Service reports that "[u]nder the 2018 Act, mandatory funding [for local and regional foods] is roughly $\$ 650$ million, more than triple in real terms the amount of funding provided by the 2002 Farm Act" (USDA ERS, 2019). One obstacle to the establishment and performance of these alternative arrangements, and to the achievement of their intended social objectives, is scaling up — that is, increasing the volume (size), value, and scope of products moved through the exchange arrangements that have emerged.

Academics whose role is to inform policymakers and practitioners, however, have no clear framework for understanding how to approach LRFSs, a term that is not consistently defined in the literature. This inconsistency frustrates efforts to examine, understand, explain, and manage important aspects of the phenomena. Much of the boundary setting has focused on geographic distance, which creates disagreement over other, overlapping conceptual boundaries and by what metrics we should measure performance. Despite several proposed approaches, current conceptual models fail to incorporate elements critical to the forms of inquiry that interest researchers across social science fields investigating the subject.

\subsection{A Framework for Understanding AFSs}

In the first part of this work, I examine the literature on AFSs and develop a multilevel framework to define and understand AFSs. This enables us to more clearly delineate the origins and objectives of AFSs and to distinguish systems seeking various forms of alterity within the paradigm. I then apply this framework to the case of "local foods." This application integrates two disparate but related bodies of literature-on LRFSs in the United States and on short food supply chains (SFSCs) largely in Europeto provide a way out of the trap consistently encountered by the former: how to form 
boundaries around "local." It becomes clear that the systems discussed in the LRFSs" approach are sets of socioeconomic arrangements that effectively consist of SFSCs. The literature under the SFSCs' approach thus presents a more coherent conceptual framework, with more neatly defined boundaries, also measured under multidimensionality and such dimensions relying on relativistic assessments of proximity. On the basis of observations made from the existing literature, this integration holds SFSCs as the "matter" or "substance" of which LRFSs are comprised. This reconception of LRFSs incorporates a multidimensional view over several measures of proximity and thus is more flexible. The framework I propose views systems as networks of agents, thus focusing inquiry on these agents and their connections.

\subsection{Applied Science: Understanding the Ecology of LRFSs to Address the Scaling- Up Challenge}

Noting the similarity between these networks of the social world and those discussed in the biological field of ecology, I turn to an allegory whereby the concepts and tools of ecologists can be used to identify ways to understand and manage the social world to achieve specific objectives. Such an allegory sees the socioeconomic networks — what I term econosystems 1 — between agential types as the ecology to be managed. At the core of any ecological approach, however, is the need to classify agents into meaningful groupings or kinds. In biology, the basic unit of such analysis is the biological species concept, which delimits populations of beings into epistemologically (and, arguably, ontologically) distinct sets. In ecology, the species is a node in the network of a given ecosystem - beings of a given species play specific roles within the

\footnotetext{
${ }^{1}$ Continuing the allegory to biological sciences through a play on the words "economics" and "ecosystems."
} 
systems as resources in the environment are cycled into energy. In the organizational sciences, however, we have yet to form so consistent a method of classification as the biological species concept. We have no tractable method for delimiting populations into meaningful groups that is not artificial. Thus, to fully apply an ecological approach to managing econosystems, I develop the Organizational Species Concept (OSC) and provide a means to operationalize it.

This OSC is then applied to show its usefulness in policymaking to manage econosystems; earlier, I noted that one policy objective is to increase the scale of LRFSs and toward addressing this challenge that the proof of concept I provide here is targeted. In other words, I take an ecological approach to investigating the agential types that may increase scale within LRFSs. It is worth noting that the establishment of new arrangements that hybridize between alterity and conventionality have been identified as a strategy to achieve scale in LRFSs. The focus is specifically on a newly emergent organizational population that carries the positive economic features of a channelcoordinating intermediary while maintaining alterity-defining "social consciousness" characteristics. These economic organizations go beyond neoclassical theory of the firm; they aim to optimize objective functions that incorporate not only profit but also utility, or some form of group or social welfare. They have been termed "food hubs" colloquially, and I refer to them in this work as food hub organizations (FHOs).

Given that FHOs and the socioeconomic arrangements that include them are nascent, little work has yet been completed in the theoretical and empirical realms to further understand their economic functions, their structures and behaviors, their ability to enact the elements of alterity that define the intended arrangements, nor the differential 
effects on the intended goals — such as scaling up — of the intermediated transactional structures they represent. Does intermediation through an FHO serve a specific kind of supplier in accessing and capturing the "local premium"? Are FHOs homogenous or heterogeneous in their structures, functions, and arrangements they create? Given the emphasis on place and location within LRFS paradigms, are there adaptive components of economic agents (both primary agricultural producers and FHOs) and the arrangements between them that prevail in specific economic environments? These questions, at their core, require understanding the interrelations between different kinds of economic agents and how connections between populations of these kinds affect the functioning of the systems in which they are found - in other words, they are ecological questions.

\subsection{Fundamental Science: Addressing the Classification Problem in the Theory of Organizational Evolution}

The OSC I develop has implications for fundamental topics in organizational science beyond its usefulness to an ecological approach for applied science directed at informing policy. Within the foundational literature of organizational theory, there is interest in understanding how new organizational forms emerge and how they thrive or fail. Such questions and theories fall under two fields: organizational population ecology and organizational evolution. Pitfalls from initial attempts in these two research streams to address the classification problem are addressed in subsequent chapters, but two are immediately pressing. First, none of the approaches correctly capture the social ontology of organizations. Of the two central developments on organizational classification, one ignores the ontology of social objects entirely, whereas the other implicitly adopts a model that does not match what modern philosophers contend about the subject. Second, 
this latter approach fails in that it incorporates as a classificatory feature a process of selection. In other words, classifications that result from its application would necessarily support a specific theory of selection and thus be meaningless as an avenue to validate theories of change.

Because no cogent, philosophically sound concept exists on how to delineate boundaries between organizational forms and thus identify meaningful populations, enquiry in these fields is frustrated; no proper classificatory concept means no ability to test evolutionary theories that would explain organizational form emergence and the import of population dynamics in ecological change. Addressing these questions requires a robust system of classifying and grouping the organizations of interest that goes deeper than single dimensions such as size, volume, legal structure, and product characteristics; organizations are similar to organisms in that both are complex in structure and defined by the interaction of multiple facets. In addition to accounting for complexity, any classificatory concept must avoid including as a basis for categorization the same mechanisms and processes that one might believe cause adaptation or explain origins of the form. These and other facets are incorporated into my development of the OSC.

\subsection{Outcomes}

I accomplish six aims in this dissertation. First, I develop a framework for understanding AFSs and apply that framework to delineate the boundaries of LRFSs, more clearly defining them and identifying avenues to examine them. Second, I create a model of classification that is more deeply rooted in the ontology of social objects. Third, I extend that model to form an OSC and offer a method to operationalize it so that I can identify distinct organizational forms. Fourth, I apply my OSC to the population of US FHOs to determine whether multiple species should be treated as distinct populations. 
Fifth, from my findings on species of US food hubs, I take an ecological approach to understanding scaling up LRFSs and offer recommendations on how this approach can guide policymakers in designing interventions. Sixth, I identify future avenues of research that build upon the foundations I establish here.

On the basis of these developments, I identify six kinds of FHOs using a national data set that captures roughly one-third of the expected total US population. I validate this set of kinds as species with descriptive names that reflect their relative role within LRFSs. Results indicate that each of the species are likely to have different effects on addressing the scaling-up challenge. Moreover, these differing effects may address different facets of that challenge; whereas some organizational forms of FHOs scale the overall system, others provide ideal incubators for the formation of new ventures at both the primary production and value-addition stages of supply chains. Some FHO species provide capacity for midscale primary producers, whereas others can increase scale by expanding local foods into disadvantaged communities through a consumer-based approach. This highlights the importance of an ecological approach to managing economic systems and has implications for policy interventions in terms of environmental conditions and targeted objectives. Because of these results, this proof of concept shows the usefulness of these approaches, and I recommend extending its application to other organizational populations within LRFS marketing channelsincluding primary agricultural producers - to complete the map of these networks and more comprehensively understand the ecology of the local foods space. 


\subsection{Tables and Figures}

Figure 1.1 Total number of academic publications per year identified under three search terms, 1990 to 2019

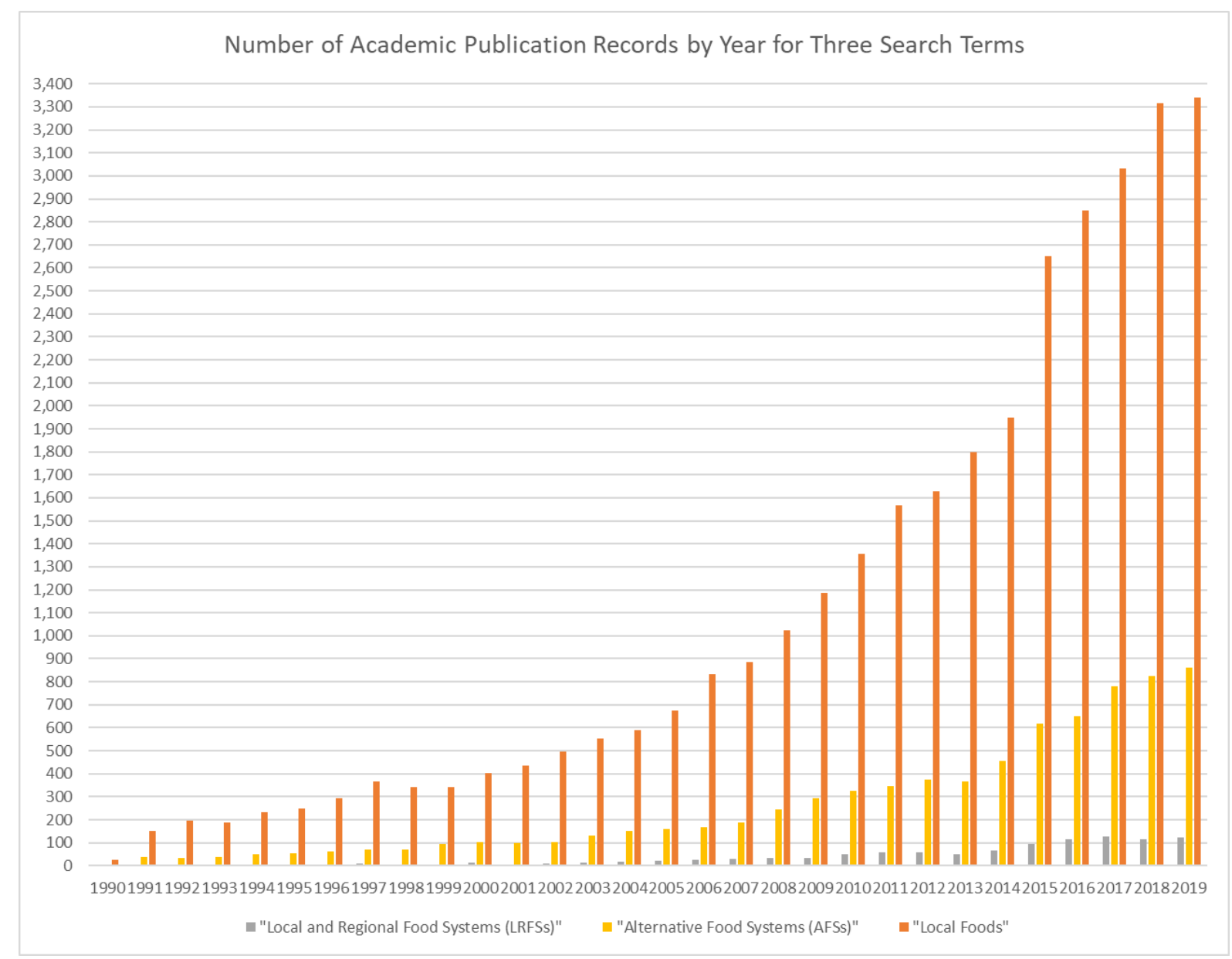

Source: Author, using data from Clarivate Analytics' Web of Science (Retrieved January 17, 2020). 
CHAPTER 2

LOCAL AND REGIONAL FOOD SYSTEMS AND THE SCALING-UP CHALLENGE

In the previous chapter, I identified that interest in alternatives to conventional, industrialized methods of producing and distributing food products has exponentially grown over the past 30 years. This interest has come from consumers, agricultural producers, civil society, local and state governments, and the federal government. It has been discussed under many banners and titles such as "alternative food systems" (AFSs), "local food," "good food." In the academic and policy literature, these terms have been treated differently, with some studies exploring, if not explicitly addressing, questions on how to increase the capacity of — or to scale up — the underlying socioeconomic arrangements that offer such products to consumers. However, no agreed-upon framework exists that allows researchers to define the boundaries between many overlapping — and at times contradictory_propositions about these phenomena. Moreover, no framework exists to guide scholars on where and how to position their inquiries. Does Study A examine the motivations of the phenomena or the resulting arrangements? To determine policies that can achieve increases in scale, how should we design Study B? When reading Study C, can we ascertain the boundaries of the investigated phenomena and therefore expect the findings to hold? Answers to these questions require a well-structured means of delimiting boundaries between different phenomena as well as defining the different mechanistic levels of any given phenomena.

In this chapter, I develop a conceptual framework that teases out the differences in the various ideas of the past 30 years. In crafting this framework, I focus on the socioeconomics of food production, transformation, and consumption under alternatives to the conventional arrangements predominant today. This approach allows me to 
separate the threads of many phenomena of interest to pertinent scholars, but the framework is general enough to be applied elsewhere. I use this approach to define the boundaries of "local foods." Within these boundaries, a frequent question of policymakers - how can we scale up local foods in our jurisdictions? — can be addressed. The framework I develop provides us an avenue for inquiry on this topic, which I detail in Chapter 3.

\subsection{Building and Applying a Framework to LRFSs}

Scholars in academia, government, and civil society have all done much work in understanding these alternative arrangements, identified by a range of terms“alternative food networks" (AFNs); “civic agriculture”; “community, local, and regional food systems"; "sustainable food systems"; "good food networks"; and "short food supply chains" (SFSCs). This smörgåsbord of conceptualizations carries a variety of meanings, structures, characters, objectives, and emphases - some complementary and others contradictory — evaluating whether these arrangements are successful in performing their intended social functions and in crafting policy interventions that adequately address their stated objectives is crucial. Using evidence from the existing literature, I use, for clarity, the umbrella term AFSs to encompass the entire array of phenomena and concepts in this space.

Two of the most well-developed concepts under this umbrella are the local and regional food systems (LRFSs) approach, predominantly in North America; and the short food supply chains ${ }^{2}$ (SFSCs) approach, largely developed in Europe. Policymakers, food system practitioners, and consumers have identified both as arrangements that are

\footnotetext{
${ }^{2}$ These are sometimes also called "short food circuits" or, in French, circuits courts alimentaires.
} 
desirable to society and worthy of investing financial, political, and social capital into. Although these two approaches may seem disparate in the literature, academic and public policy writing explicitly links them conceptually and indicates that the two concepts are worth treating jointly. Such integration is promising for identifying epistemologically relevant extensions in terms of both the conceptual framework used to describe phenomena and the methodological approach taken to investigate them.

\subsubsection{Understanding AFSs at Three Levels: Social Movements, Economic Arrangements, and Agents}

To understand AFSs, we must identify their origins and substance. What catalyzes the formation of AFSs? What do these systems seek to accomplish? And what are the important [social] objects — such as organizations, groups, and institutions - that comprise these systems? I subsequently address these questions to develop a conceptual framework made up of three levels. This framework provides greater clarity on how to approach inquiry into AFSs.

\subsubsection{Social Movements as the Catalyst for AFSs}

Many of the early developments from academic and policy spheres relating to alternative arrangements for agrofood provision have occurred organically and sporadically, as part of one or several social movements within communities. From an academic standpoint, many recent entries cite as core origins works dating to the late 1980s and early 1990s. Among these is Beus and Dunlap (1990), who, in the US context, posit that a new alternative agriculture paradigm arose as a component of the wider environmental movement of the 1960s and 1970s. Their concept is explicitly ecological ${ }^{3}$

\footnotetext{
3 “....alternative agriculture adds a critical element that was not part of past agrarian movements - an urgent concern over the ecological aspects of agriculture (Buttel et al 1986)" (Beus and Dunlap 1990, p. 595).
} 
and predominantly situated at the level of agricultural production, specifically the production practices of the farm or other primary agricultural producer. ${ }^{4}$ They elaborate this concept with a crucial note:

Most alternative agriculturalists, however, see their goals as much broader than merely reducing agricultural chemical use. Additionally, alternative agriculturalists advocate smaller farm units and technology, reduced energy use, greater farm and regional self-sufficiency, minimally processed foodstuffs, conservation of finite resources, and more direct sales to consumers (Buttel et al. 1986; Lockeretz 1986; Youngberg 1984)." (Beus and Dunlap, 1990, p. 594).

Of the six dichotomous dimensions that Beus and Dunlap (1990) propose (see Table 2.1) to differentiate the conventional and alternative agricultural paradigms, only one exclusively centers on ecology; the rest relate primarily to social and behavioral traits. Barham (1997) unequivocally recognizes Beus and Dunlap's (1990) concept as part of the compendium on sustainable agriculture ${ }^{5}$ viewed as a social movement.

The study by Barham (1997) uses "sustainable agriculture" as a cognate for “alternative agriculture." Thus, the social movements surrounding these terms are, if not the same, somehow very closely related, inclusive of the former subsumed as one element of the latter. It is important to note that in this study, I share the view of some authors (for example, Friedmann and McMichael 1989; Friedmann 1993) who connect Polanyi's analysis of the double movement of societal self-protection with "the emergence of alternative agriculture social movements that call for such things as relocalization of food production, consumption networks more closely tied to community-level institutions, environmental protection and stewardship through farming,

\footnotetext{
${ }^{4}$ Even more specifically, "[a]t the heart of any definition of alternative agriculture is an emphasis on organic or near-organic practices" (ibid., 1990, p. 594).

5 The same work also uses "alternative agriculture" as an apparent cognate for this term.
} 
and fair trade practices with the Third World" (Barham, 1997, p. 241). Barham

establishes that there is not one singular movement that seeks to counter the outcomes of conventional systems, but several.

Galt (2017) makes this assertion explicit, identifying five distinct alternative food movements: organic agriculture, fair trade, civic agriculture and sustainable community food systems, food justice, and food sovereignty. It is not yet entirely clear when (or where, or how) the discussion on these movements shifted from a narrow framing centered on agricultural production space toward a wider view on "food" or "agrofood product" space. This shift is critical because it brings managing the functions and processes that occur beyond the farm gate under the auspices of the social movement; the movement is no longer only about what happens on farm but also on what affects the product in the entire journey from planting to eating. ${ }^{6}$ Thus, here I adopt the term alternative foods.

2.1.1.1.1 "Embeddedness" as the alterity sought. The study by Barham (1997) is also notable because it (1) examines the phenomenon of interest here for the first time in a non-US context by analyzing an alterity-seeking social movement in France; (2) introduced into the conceptual landscape of such movements the Polanyian framework ${ }^{7}$ from economic sociology; and thus (3) entered into the lexicon of this phenomenon the notion of "embeddedness," making the search to (re-)embed economic transactions into social relations a central facet of the social movements being considered. Here, "embeddedness" refers to the conceptual definition from economic sociology typically

\footnotetext{
${ }^{6}$ See how Hinrichs (2000) delineates between "sustainable agriculture" and "local foods."

7 This the well-known theory posited by Polanyi in his 1957 book, The Great Transformation. For additional, contemporary discussion of this framework, also see Cangiani (2011).
} 
credited to Karly Polyani; under that framework an economic behavior is socially embedded when there are noneconomic institutions dictating constraining boundaries. Such noneconomic institutions can include things like kinship and tribal fealty, religious doctrine, political affiliation, and other moralizing values. As industrialized agrofood systems become the conventional means of production and distribution, economic transactions around food became disembedded — governed almost exclusively by economic institutions. Thus alterity — the state of being different than that which is conventional — comes from constraining economic exchange of food products and services by re-embedding transactions into arrangements where noneconomic institutions, such as social and moral values, can act as constraints.

This facet of embeddedness is worth particular attention because it appears in much of the literature on alternative foods that follows Barham (1997), playing a central role in subsequent concept development. A core subset of that literature provides extensions of the embeddedness concept and its role in defining that which is in opposition to conventional systems. Murdoch, Marsden, and Banks (2000), for example, extend the concept to see the economic activities of transactions over alternative foods being embedded not only within social relations but also within natural relations in work focused on the "turn to 'quality' in food production and consumption" (p. 107). Authors who reference this work reformulate their concept as "territorial embeddedness" (for example, see Ilbery and Maye 2005). In their case study of consumer motivations at a Canadian farmers' market, Feagan and Morris (2009) provide three spheres of embeddedness: social, natural, and spatial. 
Winter (2003) also considers embeddedness and the "turn to quality" in AFSs. Examining food-purchasing behaviors by end consumers in selected locations of England and Wales, Winter (2003) concludes that the defining aspect of alterity is not about a "turn to quality based [in] organic and ecological production [practices]," nor is it about embeddedness, but rather that it is "a defensive politics of localism." (p. 23) However, it is unclear how this is not simply a kind of place-centric embeddedness, a search for exchange that is socially embedded at the local scale is about countering globalization within food systems and challenging the global agrofood complex. Hinrichs (2003) simultaneously bolsters and contradicts the Winter (2003) approach, finding in a case study of arrangements around local foods in Iowa that although the alterity sought through localization can have a defensive politics, it may also seek alterity through the politics of receptivity and diversity. These two approaches can be in tension or contradiction with one another within the same set of arrangements. It is important to note that Hinrichs (2003) does not reject embeddedness in favor of a politics-centered definition of alterity but rather sees embeddedness as a feature of the arrangements (that is, the relationships) and politics as the motivations (that is, an aspect of the social movement).

2.1.1.1.2 Toward a more realistic understanding of embeddedness. Beyond these extensions and qualifications, the work by Hinrichs (2000) is salient, bringing general critiques about the rigidity of Polanyi's dichotomous conception to bear on arrangements of alterity in agrofood space. First pointing to Granovetter (1985), which lays the foundation for questioning this rigidity, its argument centers on that of Block (1990), which reposits embeddedness not as a this-or-that framework but as a tension. 
That tension is between full embeddedness, where social considerations completely rule transactions of exchange, and the marketness (that is, price relevance) and instrumentalism (that is, self-interest) of the neoclassical disembedded economy. Thus, Hinrichs (2000) finds a basis for reenvisioning the Barham (1997) concept, with one important caveat: investigating the Block (1990) notion in the context of local food systems comprising "direct agricultural markets, predicated on face-to-face ties between producers and consumers ${ }^{8 "}$ (ibid., p. 295). Focusing on farmers' markets and community-supported agriculture schemes, it concludes that in these two arrangementsand in local food systems in general—marketness and instrumentalism are qualifiers on, or are in tension with, embeddedness, in line with Block (1990).

Sage (2003) enriches the Hinrichs framework for alternative foods ${ }^{9}$ by integrating into it the relations of regard concept developed by Offer (1997) and Lee (2000). "In contrast to neo-classical market exchange where personal acquaintance is immaterial and the material gains from trade are all that matter," Sage (2003) notes, "reciprocal exchange ${ }^{10}$ embodies a 'process benefit, usually in the form of a personal relationship."” (p.48, quoting in part from Offer 1997) For Sage (2003), this concept of regard "illustrate[s] the benefits to both parties arising from their interaction that go well beyond narrowly financial evaluations" (ibid., p. 47). In other words, relations of regard explains why embeddedness matters: a sense of connectedness - in the context of Sage (2003), "personal interaction" between seller and buyer-is a source of satisfaction that may

\footnotetext{
8 "Such direct market venues as farmers' markets, community-supported agriculture, vegetable box schemes, and other cooperative distribution and delivery programs..." (ibid.)

${ }^{9}$ The study by Sage (2003) relies specifically on the notion of "good food," which the author attempts to define, positing this as exchanged through "alternative food networks."

${ }^{10}$ This "arises out of the intrinsic benefits of social and personal interaction, from the satisfactions of regard.[and is] preferred when trade involves a personal interaction, and when goods and services are unique, expensive, or have many dimensions of quality." (Offer, 1997, p. 450, as cited in Sage 2003).
} 
"enable sub-optimal production and exchange"” (Lee 2000, p. 139, as cited in Sage 2003). "The buyer (consumer) discounts the uncertainties, idiosyncrasies and usually higher prices associated with small enterprises heavily reliant upon the labour of their owners. For, in addition to the desired product, the buyer gains insight into the production system, status and identity associated with the consumption of a good with limited distribution, and enhanced expertise" (ibid., p. 48).

\subsubsection{Systems as Networks of Agents}

Initial concepts of alternative foods focused on social movements. However, academics quickly shifted to simply discussing "systems" and "networks," in some cases even "supply chains," often using these terms interchangeably with one another, and, crucially, conflating them with the social movement. Barham (1997) provides some basis for this shift, noting a network structure to the social movements. Within the social movement is a set of actors, each with their own sociopolitical motivations and aspirations. Each actor's desires may be distinct from those of others. Through the social movement, however, they link together. This linkage catalyzes the actors to recognize a common denominator upon which they can mutually and collaboratively act. That catalyzation eventually leads to a change in the social, political, and/or economic environment. This concept of network is applied at the level of the social movement, but other network conceptions are applied at a different level: that of the routinized arrangements of exchange that seek to enact the core notions of alterity ${ }^{11}$ codified within the social movement. Relatedly, Watts, Ilbery, and Maye (2005) advocate for focusing on

\footnotetext{
${ }^{11}$ Here, replacing conventional ways of being and doing with those that [are perceived to] mitigate or eliminate the root causes of concern by adherents to the social movement.
} 
these arrangements - rather than the products themselves - as well as on the "networks through which food passes" a "stronger" approach to "alternative systems of food provision" and labeling those centered on product attributes such as "food relocalization" and the "turn to quality" as "weaker" (ibid., pp. 22, 27-36).

Weber, Heinze, and DeSoucey (2008) explain the importance of distinguishing between the social movement and the resulting artifacts as well as examine how the movement for grass-fed (that is, a specific production practice with environmental and cultural attributes) creates the opportunity space for establishing new markets or market segments. It posits that social movements can enable surmounting three challenges by (1) creating entrepreneurial producers (supporting motivation, commitment, and innovation), (2) creating collective producer identities, and (3) establishing regular exchange between producers and consumers. Here, I stress the second item given that these collective identities "give rise to cooperative efforts to institutionalize the market category" (ibid., p. 547, emphasis added). Of note, the work references evidence in the literature (specifically, Carroll and Swaminathan 2000) that, as part of the cultural change, social movements often lead to the creation of entirely new organizational forms, which are one method of legitimizing and communicating the emerging identity. Weber, Heinze, and DeSoucey (2008) do not discuss how these identities shape the choices on the structure and nature of the resultant organizations and the ways in which they are arranged or linked to perform the functions required for value creation and for the exchange of goods.

\subsubsection{Proposing a Multilevel Model for Greater Consistency and Clarity}

The logic of what precedes can be summarized as follows: social movements arise in cultural and political spaces, voicing a collective desire for an alternative that solves a perceived social failing or absence. This creates an opportunity space into which agents 
seeking to enact the alterity sought by the movement can enter or form. Some of these agents adapt to the space, whereas others are designed anew within it. These agents form transactional arrangements with patterns of behavior in line with the alterity of the movement. The set of these arrangements and patterns coalesces into a systemprocesses of doing and being that center the alterity at the heart of the precipitating social movement.

From this, one can identify different levels of interest to understanding the phenomenon. First, from works such as Weber, Heinze, and DeSoucey (2008), the social movement may be epistemologically—if not also ontologically—distinct from the set of arrangements that arise to enact the alterity it seeks. Furthermore, these arrangements of alterity are by and between different agents who form relationships of exchange by and between one another. These agents include both individual natural persons acting as producers (in sole proprietorship) and as consumers, as well as organizations (that is, legal persons) and collectives. Thus, I identify at least three levels of epistemological significance:

1. A macro level that is the social movement, where there is the formation of an "agenda" and opens an enabling environment;

2. A meso level that is the set of arrangements that arise to enact the goals of the movement; and

3. A micro level of the atomistic agents that form those arrangements through their exchange relationships.

The macro level (social movement) is where imaginaries ${ }^{12}$ are established and where concepts and desires are formed by a collective within society. In the Weber, Heinze, and

\footnotetext{
${ }^{12}$ In this work, I use a [loose] application of a concept developed in sociology, philosophy, and psychology, especially in the European literature. Generally speaking, the concept signifies the set of meanings and values conceived of by some social group. For a concise backgrounder, consider the
} 
DeSoucey (2008) framework, it is where opportunity space is created. The meso level (arrangements) consists of forming structures within that space that enact the tenets of the world view established at the macro level, what I call arrangements of alterity. These arrangements of alterity are made up of relationships of exchange between agentsindividual natural and legal (that is, organizations) persons - that lie at the micro level. Figure 2.1 displays this concept, incorporating additional developments that follow.

\subsubsection{Embeddedness and Alternative Arrangements of Exchange}

At the meso level, the set of arrangements are known in the literature as "network," "system," "supply chain," "food chain," and several other terms. These are nearly always used interchangeably or positioned as conceptually synonymous. Many of the seminal pieces that focus on these arrangements most frequently adopt a "network" concept as the focus, using the others terms as synonyms. Researchers (for example, Renting, Marsden, and Banks 2003; Sage 2003; Winter 2003; and Barbera and Dagnes 2016) refer to Murdoch, Marsden, and Banks (2000) as having developed the network concept of AFS. Although this work has been previously noted here for its extension of embeddedness, its most meaningful contribution is that it lifts Goodman's (1999) initial application of actor-network theory (ANT) ${ }^{13}$ to agrofood studies into greater prominence

following blog post by Nerlich: http://blogs.nottingham.ac.uk/makingsciencepublic/2015/04/23/imaginingimaginaries/ (viewed February 2018).

13 "In the ANT framework, networks are built from relations or associations, in which it is the links or ties between the component parts that confer agency rather than any essential (natural, social) characteristics held by a particular subject or object. [...] network activities or network outcomes can only be fully comprehended by taking into account the full range of interrelationships found therein. Agency is understood as the collective capacity of heterogeneous networks in which the activities of nonhumans may count for as much, if not more, as the activities of humans. In actor networks, action depends on relations... ANT focuses merely upon the length of the networks and tends to work on the assumption that power equals length of network reach (Latour 1987; Murdoch 1995). It uses the same framework of analysis for both long and short networks and seeks to show all that is involved in establishing networks and the 
and then links this framework with conventions theory. ${ }^{14}$ It is important to use

conventions theory's tools to qualify actor networks and the embeddedness they offer; or, more explicitly, to "determine different degrees [and qualities] of embeddedness, the varying shapes and compositions of networks, [explain how they developed and moderate different types of exchanges], and the scope for significant innovation" (ibid., p. 116). Through this integration of theoretical frameworks, Murdoch, Marsden, and Banks (2000) propose that we "begin to consider the construction of economic networks" (ibid., p. 113) within which the exchange so-called "good food," and thus the intended alterity of the social movement, is achieved.

Sonnino and Marsden (2006) revisit the centrality of embeddedness to AFNs; recognizing that AFNs had since been "[v]ariously and loosely defined in terms of 'quality,' 'transparency,' and 'locality”' (p. 181), the authors argue that such concepts are inadequate foundations upon which to base analytical approaches to AFNs because they are highly contextualized, are contested, and fail to differentiate between what is conventional and what is alternative. Noting that "a key characteristic of the new supply networks [i.e. AFNs] is their capacity to re-socialize or re-spatialize food" (ibid., p. 183), the work advocates using a holistic concept of embeddedness as the analytical difference

\footnotetext{
amount of work that is required in holding the various alliances, associations, and relations together" (Murdoch, Marsden, and Banks 2000, pp. 112-113).

14 "According to Wilkinson (1997a) [...] conventions theory has developed from an analysis of the rules, norms, and conventions that undergird the wage relation in contemporary capitalism. It recognizes that "not only labour but commodities in general suffered the deficiencies of 'incomplete contracts," requiring therefore rules, norms and conventions for their production and exchange" (Wilkinson 1997a, p. 309). The theory has thus been extended to examine the underlying systems of negotiation that configure modern economies. Like ANT, it sees productive activity as a form of "collective action" (Callon 1998; Storper 1997) that relies on coordinating various entities within some type of action framework (network, filiere, chain). At the heart of this collective action are conventions, defined as "practices, routines, agreements, and their associated informal and institutional forms which bind acts together through mutual expectations" (Salais and Storper 1992, 174).” (Murdoch, Marsden, and Banks 2000, p. 113).
} 
maker upon which to draw the boundaries between alternative and conventional. This holistic view of embeddedness uses extensions of embeddedness that surpass the social realm and integrates relations of regard and conventions theory "to account for both the horizontal and vertical embeddedness of [AFNs]." (ibid., p. 190) Drawing from these theoretical developments — especially that of Sonnino and Marsden (2006)—Higgens, Didben, and Cocklin (2008) use a case study of an environmentally friendly production label to examine how certification and labeling may form the basis for embeddedness in extended exchange relationships. That case study concludes that the third-party certification aspect of the labeling scheme, although building trust and transmitting information about the environmental outcomes of the production practice (both aspects of embeddedness), could go only so far; to overcome initial barriers, the AFN in question still had to rely on "proximate [intermediary] actors to make the certification work" and enable the establishment of an extended AFN (ibid., p. 25).

Some concepts of AFNs do not explicitly turn on this holistic embeddedness. Venn and others (2006) efficiently summarize the various approaches in the prolific European literature of the early 2000s. But it is evident in this review of such competing definitional approaches that most of them can be easily located as an element already within the holistic embeddedness framework that predominates. Similarly, Feagan and Morris (2009) cite several examples of North American and European studies of farmers' markets (ibid., p. 236). Tregear (2011) also offers a review of the AFN literature, denoting three perspectives, "each tend[ing] to adopt ontological and methodological approaches [to AFNs] distinct from the others" (ibid, p. 420). The first of these is a political economy perspective "conceptualizing AFNs as movements in constant struggle 
against threatening global capitalism" (ibid., p. 420, emphasis added). However, this perspective conflates the macro and meso levels; I carefully distinguish the two levels in my framework. The two central works Tregear (2011) denotes as the core of this perspective predate the study by Weber, Heinze, and DeSoucey (2008). The other two groupings offered by Tregear (2011) — a rural sociology/development perspective and a modes of governance/network theory perspective - draw on almost the same set of conceptual developments discussed earlier that led to a holistic embeddedness approach to AFNs. Thus, having rightly placed the political economy perspective at the macro level and having seen that the other two largely appear to have been unified yields a concept of the meso-level's arrangements characterized as those exchange relationships, or transactions, that are embedded.

\subsubsection{Adopting a Systems Definition of the Meso Level}

A coherent definition is thus needed. However, given the distinction between the macro and meso levels, the predominance of network-centric terminology across both levels is problematic. Here I exclusively adopt the term system, knowingly disregarding the discussion by Lockie and Kitto (2000), which contrasts the systems of provision and ANT frameworks as used in the sociology of food literature. Alas, I will not be the first in the extant body of literature to disregard their careful sociological delineation. In the case at hand, the arrangements of alterity perform a certain set of functions that are still effectively the same as those for conventional foods, the difference maker between the two not being the functions themselves but rather the process and manner of performing them - that is, the degree of embeddedness in the performance of these functions. The literature on conventional foods ubiquitously uses the term system to denote the set of connections between economic agents that perform these functions. Moreover, the 
alternative foods literature also ubiquitously conflates multiple terms. Thus, I propose the following definition:

An alternative food system is the set of arrangements among institutions, organizations, and other socioeconomic agents that produces, transforms, distributes, and consumes food products in a manner that intends to conform in some central aspect to the imaginary of alterity proposed by some alternative food movement.

Given that some authors differentiate between alternative food social movements, so too are delineations made among systems that are established within the opportunity space created by a movement. Although they may overlap, these systems are nonidentical: "What they share in common is their constitution as/of food markets that redistribute value through the network against the logic of bulk commodity production; that reconvene 'trust' between food producers and consumers; and that articulate new forms of political association and market governance" (Whatmore, Stassart, and Renting 2003, p. 389). Figure 2.2 provides a graphical representation.

It is crucial to stress that the two systems are different; much of the literature, especially on AFNs, conflates the distinct levels of the general case of alternative and the specific case of local. Consider the following excerpt from Wald and Hill (2016):

Regardless of the theoretical approach employed, 'AFNs seek to localize food systems and to encourage contact between food producers and consumers, seeking to respatialize food systems perceived to have become 'placeless' (Harris 2010, p. 355). A focal feature of AFNs, and especially within activist discourse, has often been their 'local' affixation (Goodman et al. 2012). Therefore, AFNs are conventionally perceived as 'local food networks' (LFNs). The 'localisation' of food systems is seen as standing in a stark contrast to the mainstream agro-industrial and global food system, analysed by McMichael (2009) as a 'food from nowhere' or a 'corporate' food regime. Discourses of LFNs, then, situate food in a place and advocate for a respatialisation of food systems (Feagan 2007). This reterritorialisation and spatial reconfiguration of food systems bring to the fore the geographic attributes of food production and consumption. The geography of local food systems, however, is only one key aspect. Apart from being rooted in a place, LFNs 'aim to be economically viable for 
farmers and consumers, use ecologically sound production and distribution practices, and enhance social equity and democracy for all members of the community' (Feenstra 1997, p. 28). Implicit to this aim are beliefs that a globalised system is not and perhaps cannot be economically fair, environmentally sustainable and socially just (ibid., p. 206).

Much of the earlier discussion suggests hints of this conflation of alternative and local, but the references there, along with those cited in the Wald and Hill (2016) excerpt, are not exhaustive. ${ }^{15}$ This is problematic in light of the literature that denotes distinct movements with distinct motivations. Thus, there are distinct opportunity spaces, distinct markets, and distinct arrangements of alterity. Many of the various movements are defined by imaginaries that seek alterity in production practices (for example, organic, grass-fed, pasture-raised, and eco-labels), justice and equity (for example, fair trade, humane treatment of animals), and special quality assurances (for example, geographic and typical product certifications, non-GMO, and quality signs such as Label Rogue, or "Red Label"), where local does not feature as the difference maker (although some may be about locality). For example, Winter (2003) and Hinrichs (2003) posit concepts of the local and regional food movement that are distinct and independent from those with specific production practice attributes.

\subsection{Local and Regional Foods: Achieving Alterity through a "Commitment to Place"}

Despite the indisputable aspect of localness or relocalization in the wider alternative foods space, local foods and local food systems and other such terms are used in contexts denoting products with provenance- or source-identification as a concept that is ontologically distinct from other components discussed as being "alternative." This is

\footnotetext{
${ }^{15}$ For additional examples, see Lamine and others 2012; Eriksen 2013; Venn and others 2006; and Tregear 2011.
} 
the case in Europe to a degree but is especially so in the North American context. Given the aforementioned framework, we must first establish whether there is a distinct social movement centered explicitly on the localness of agrofood products or whether this is simply a means to an end. to establish the macro level and set the boundaries of the opportunity space in which a distinct AFS would be created, the questions to answer are "Is there a local foods movement?" and "If so, what is its defining imaginary?" This inquiry is necessary.

\subsubsection{Establishing a Distinct Macro Level}

Some researchers have expressed uncertainty on whether local constitutes a distinct movement. As Starr (2010) ponders, "Is local food a set of policies, a consumer fad, a new market, or a social movement?" For it to be the latter, Starr (2010) asserts that it must meet two thresholds: (1) formulating a "we" (a common identity) that is sustained and (2) creating a "new idea." Ultimately, she concludes that the thresholds are met and that there is a local food social movement. Of course, this is only one conception of the elements defining a social movement; Weber, Heinze, and DeSoucey (2008) provide a succinct discussion of alternative ways to conceiving the constituent elements. Starr's (2010) analysis is one of the most in-depth as it explicitly treats the local food movement, but other conceptual approaches to social movement boundaries lead to the same conclusion. I propose a minor expansion to "local and regional food movement" (LRFM). This adopts some of the expanded nomenclature common to both the emerging literature dealing with the micro level of the phenomenon (that is, that on new organizational forms, such as food hubs) and the general preponderance of approaches 
within the policy community. ${ }^{16}$ Granted, even adopting this expanded naming convention does not delimit its motivations and objectives.

However, delineating the imaginary of this movement is not a straightforward exercise; an almost unanimous approach among academic and policy researchers has been to note that definitions of local and local foods are ill-defined, in general lacking consensus, being fluid, contested, and/or depending on context, leading to with competing, and sometimes contradictory, meanings (Feagan 2007; Darby and others 2008; King and others 2010; Martinez and others 2010; Eriksen 2013; Pinchot 2014; Low and others 2015; Thilmany 2015). Often, local food appears in academic literature without an explicit definition. ${ }^{17}$ In addition, conflation across various levels and terms is rampant, leaving one to infer motivations of the movement (macro level) from the literature speaking at the level of systems and transactions (the meso level) and organizations, institutions, and products (the micro level). Likewise, inference may also need to be made about the local food movement from the literature that attaches ${ }^{18}$ terms such as "alternative" and "civic," while defining the phenomenon in terms of "localization," "local," "community," and so forth. Thus, here I adopt an all-comers approach to identifying the particular imaginary of the LRFM.

\subsubsection{Delineating the Imaginary of the Local and Regional Foods Movement}

Several recent studies provide detailed accounts of the various approaches used to construct concepts of local foods (Johnson, Aussenberg, and Cowan 2013; Eriksen 2013;

\footnotetext{
${ }^{16}$ For examples, see Martinez and others 2010; Low and Vogel 2011; Low and others 2015; and Berti and Mulligan 2016.

${ }^{17}$ For examples, see Guptil and Wilkins 2002; Nost 2014; and LaForge, Anderson, and McLachlan 2017.

${ }^{18}$ Or, arguably, misuses and conflates.
} 
Pinchot 2014; Low and others 2015; Thilmany 2015). Reducing geospatial distance between the places of production and consumption tends to be a predominant feature. This is especially the case in early literature discussing local foods. (King and others 2010; Martinez and others 2010) Under this approach, "local" is about producers and consumers being from within some arbitrarily defined radius of each other —in other words, place a dot on a map and measure out 50 miles, or 100 miles, or 400 miles. However, radial distance is not the only approach to a geospatial definition. Some have considered the total distance traveled by a single food item (so-called "food miles") in the course of production, transformation, and distribution processes. Others use political jurisdiction boundaries as a proxy for geospatial distance.

What metric and boundary qualifies as sufficiently proximal, and who gets to determine this, is a matter of great contention among academics, policy officials, and actors alike. Some researchers have presumed that end consumers are the final arbiters (Coit 2008; Thilmany 2015), but any approach that favors one group over another fails to appropriately account for the fact that social movements and the creation of their imaginary are driven by a network of actors that includes other components of society. Key to all of these geospatial approaches is a desire is to reduce the distance between producers and consumers, relative to those [often vast] distances found in conventional, industrialized arrangements. Thus, one central emphasis of the LRFM imaginary appears to be a relative reduction in the geospatial scale of food. Despite the concerns over being overly exclusive, the consumer-centric research, in addition to that with other groups of actors, including primary agricultural producers, provides evidence on one salient point: the exact dimensions of this geospatial down-scaling are context dependent along the 
lines of geography, population density, product type, agroclimatic conditions, and social and economic infrastructure.

Reducing distance and increasing proximity between production and consumption activities on the basis of geospatial metrics are not the only posited elements of the alterity sought within the local foods space. As Martinez and others (2010) note, "[i]n addition to geographic proximity of producer and consumer... local food can also be defined in terms of social and supply chain characteristics" (ibid., p. 3). Nearly all of the works that conflate "localization" with "alternative" referenced in the section here on AFSs implicitly emphasize this social distance aspect. ${ }^{19}$ In this vein, one can identify a recent shift toward an imaginary of local foods that seeks alterity through social interaction, knowledge, and trust. At times, this is out of a pragmatic methodological approach by researchers to evade the problematized geospatial definition (Martinez and others 2010; Low and Vogel 2012; Pinchot 2014), but for others it has been driven by direct empirical observation of actor conceptions (Hinrichs 2000; Nurse, Onozaka, and Thilmany McFadden 2012). Again, this is about relative distance, in relation to the conventional, and depends on context. This social and economic proximity maps well onto the unidimensional notion of embeddedness in the early literature on alternative food movements, such as the Barham (1997) attribution, which saw it as the alteritydefining element of a more generalized alternative food movement.

However, these central reviews also make it clear that elements beyond relative geospatial and social proximity often enter into the local foods imaginary. Martinez and

\footnotetext{
${ }^{19}$ It is notable, too, that many of these are from the European perspective and/or written by geographers and sociologists.
} 
others (2010), for example, denote concepts of local that incorporate (1) production methods and distribution practices that reduce environmental impact; meeting standards of (2) fair labor and (3) animal welfare; (4) "provenance," which is defined therein as aspects of who produced the product including the personality and ethics of the grower, attractiveness of the farm and surrounding landscape, and the story behind the food; and (5) the size and scope of primary agricultural producers. Even Starr (2010) conflates as one the local and the "organic." Meanwhile, much of the European literature that mentions local does so in a context of a new rural development paradigm, where [re]localization of food is seen as a way to increase the capturing of value by communities that have become economically marginalized under processes of industrialization (Murdoch 2000; Renting, Marsden, and Banks 2003; Wiskerke 2010). So many facets can be problematic, especially when countermanded by the approaches of others that attempt to neatly differentiate between such elements. Qualities such as "grass fed," "organic," "humane," and others can still be attributes of the movement of interest, but are they defining attributes? As the literature on the alternative food movement shows, these attributes are surrounded by their own movements, and having networks and concepts that overlap does not invalidate their distinctiveness. ${ }^{20}$

\subsubsection{1 "Commitment to Place" as a Unificatory Imaginary}

What if there were a more robust conception of the LRFM's imaginary under which all of these features could be subsumed —one that might hold promise to alleviate the contention that has thus far frustrated researchers, policymakers, and practitioners

\footnotetext{
${ }^{20}$ The Complex Adaptive Systems framework discussed by Stroink and Nelson (2013) helps to conceptualize this. Under this framework, the local and regional foods, organic, and grass-fed (among others) movements are the social coalitions that form to establish distinct but parallel or layered systems nested within a wider alternative food system.
} 
alike? A novel approach to delimiting an otherwise overly heterogeneous imaginary comes from work published just within the past two years, in research focused at the meso and micro levels of the local foods space. In a 2015 Report to Congress, a team of USDA research staff offer a definition of LRFSs as "place-specific clusters of agricultural producers of all kinds — farmers, ranchers, fishers—along with consumers and institutions engaged in producing, processing, distributing, and selling foods." (Low and others 2015; p. 1, emphasis added). In the same year as this governmental report, Fischer, Pirog, and Hamm (2015) enter the academic literature, focused on refining the definition of food hubs, an emergent organizational form attributed expressly to local food systems. Food hubs, the work contends, "are, or intend to be, financially viable businesses that demonstrate a significant commitment to place through aggregation and marketing of regional food" (ibid., p. 97). In part, this definitional approach is based on empirical observation of the organizational population through data on mission statements. ${ }^{21}$ Fischer, Pirog, and Hamm (2015) articulate an explicit understanding of "place," saying that "[t]his term uses Hudson's idea of place as a construct composed of both the production and consumption of material objects as well as social relations within a region" (p. 97, emphasis in original). This articulation continues by stressing that place is not simply a space within physical geography but also entails the active "creation of attachments to a community as the location of the socialization process and as a shaper of identity." Clearly, "place" is a concept that retains the kind of fluidity commonly denoted

\footnotetext{
${ }^{21}$ The most recent report for the data series used by Fisher, Pirog, and Hamm (2015) in the formulation of their definition of food hubs concludes that "commitment to community through the distribution of locally and regionally produced foods is the critical value that separates food hubs from other businesses occupying the same food supply chain space" (Hardy and others 2016, p. 46).
} 
as a key feature of local foods, as does "commitment." In such an imaginary, placeness is entailed by the social, geographic, and cultural elements commonly noted in the local foods literature, whereas elements such as social justice, economic fairness and prosperity, environmentalism, health improvement, nutrition, and education involve committedness. This fluidity allows the alterity sought by this imaginary to be relative and contextual, key features also consistently identified.

\subsubsection{Dimensions of Proximity: Operationalizing "Commitment to Place" to Define LRFSs}

Eriksen (2013) proposes defining local food using three dimensions of proximity: geographic proximity, relational proximity, and values proximity but does not develop them well in relative terms. It is crucial that these dimensions map over the previously noted holistic embeddedness concept. The study by Eriksen (2013) explicitly denotes embeddedness in its discussion but fails to address the systems component and thus ignores the role of the structural arrangements through which such proximity is achieved, economic value is created and transmitted, and food products with attributes of alterity are conveyed.

In addition, Eriksen (2013) draws solely upon the discussion of that concept offered by DuPuis and Goodman (2005), and thus subsumes it as some "additional [element]" under the dimension of Values Proximity. Applying Eriksen's (2013) dimensions to a more complete understanding of the embeddedness concept that forms the alterity in agrofood space shows that the measures of [relative] proximity are likely good candidates to be approximations for various forms of embeddedness. Thus, the commitment to place imaginary of the LRFM is, I argue, about the weighting of degrees

of embeddedness, measured using dimensions of proximity, such that there is moderate to 
high values proximity in tandem with a high degree of geographic proximity and/or relational proximity. This delineates in a meaningful way the opportunity space that is created by the LRFM and thus insight as to what the characteristics for the constituent arrangements established within it. It also addresses one of the persistent concerns expressed in the literature: fetishization of geospatial proximity as being the only route to achieving embeddedness (Hinrichs 2000; Goodmann 2004; Born and Purcell 2006; Higgens Didben, and Cocklin 2008). Effectively, definitional approaches that ignore the other dimensions and favor only geospatial distance simply use a satisficing marker for the set of place- or community-based notions of alterity, and the mix of socioeconomic objectives, held by actors within the LRFM.

Given the general conceptual framework developed in this chapter, one can then define the meso level by substituting particulars into the general definition of an AFS:

A local or regional food system is the set of arrangements among institutions, organizations, and other socioeconomic agents which produces, transforms, distributes, and consumes food products in a manner that intends to conform to a commitment to place via increased relative proximity within at least two dimensions.

As noted in the introduction, Eriksen (2013) does not focus on development of the structural arrangements (the meso level) used in achieving high degrees of relative proximity, and thereby enacting the alterity sought by the LRFM. This begs the question: What do we know, if anything, about these arrangements? In other words, what structures have come to fill the opportunity space created by the LRFM, fulfilling the Hinrichs model of embeddedness (as conceived by the LRFM's imaginary) balanced with the instrumentalism and marketness that are inherent in economic transactions and exchange relationships? Literature from both the largely North American local and regional foods 
and largely European AFNs threads points to an extant conceptual model for these arrangements: SFSCs.

\subsubsection{Understanding the Meso Level: SFSCs as the Substance of LRFSs}

In the preceding section, I noted that a number of recent pieces in the literature from North America adopt an approach to defining local foods that avoids the problematized geospatial approach to distance, adopting a social distance measure instead. In that discussion at the macro level, this social distance element was all that was important. But the details of these works (unwittingly) also offer meaningful insight as to the meso level, where a reference to "supply chain characteristics" (Martinez and others 2010 , p. 3) was made. The details of the definitional approach taken to "local foods" or “local food supply chains” by King and others (2010), Martinez and others (2010), Pinchot (2014), and Low and Vogel (2012), among others, is explicitly one that centers on such characteristics. These adopt the concept of SFSCs as the difference maker that defines "local." The SFSCs concept that is relied upon in these works is primarily as developed by Marsden and Banks with other colleagues in the European context (Marsden, Banks, and Bristow 2000; Renting, Marsden, and Banks 2003). Thilmany (2015) denotes Nurse, Onozaka, and Thilmany McFadden (2012) and Hinrichs (2000) as providing empirical support that consumer agents explicitly use the structure of marketing channels, specifically "shorter" supply chains, as a proxy for "local." This SFSCs approach is, however, a description of the arrangements comprising LRFSs, not the imaginary of the movement, easily and commonly conflated. But at points, these and 
numerous other works ${ }^{22}$ do rightly and unequivocally tie the SFSC concept as the core substance of LRFSs. When one fully explains the SFSC concept, the importance of this tie emerges more clearly.

Marsden, Banks, and Bristow (2000) and Renting, Marsden, and Banks (2003) pick up where Murdoch, Marsden, and Banks (2000) left off. The 2003 work, for example, notes that its intent is to "explore[s] different dimensions" of and "specify empirically different types of" AFNs, which I have rebranded as systems to differentiate more clearly between macro and meso levels of the phenomenon. Three "mechanisms" of “organizational structure" (here meaning arrangements between individual agents) are delineated. Table 2.2 reports these, including examples from the original authors, and provides the terms commonly used in the North American literature on local foods associated with each. As Table 2.2 shows, most of the North American literature contends that the third form of SFSC proposed by Marsden, Banks, Bristow, and Renting (2000) are not legitimate arrangements under the local foods ethos; however, this is still a matter of some debate. ${ }^{23}$ Much of the aforementioned North American literature ignores the fact that Renting, Marsden, and Banks (2003) contend that these mechanisms are only one of two dimensions by which SFSCs are comprised:

The specific quality definitions and conventions involved in the operation of new food networks are a second dimension differentiating empirical expressions of SFSCs. All SFSCs operate, in part at least, on the principle that the more embedded and differentiated a product becomes, the scarcer it becomes in the market (ibid., p. 401, emphasis in original).

\footnotetext{
${ }^{22}$ For some examples, see Ilbery and Maye 2005; Feagan 2007; Clancy and Ruhf 2010; Abatekassa and Peterson 2011; and Santini and Gomez y Paloma 2013.

${ }^{23}$ For the time being, I remain agnostic on this point, but the conceptual and definitional framework proposed here may have new meaning in this debate.
} 
The work proposes two such "quality definitions" in terms of "paramountness": regional or artisanal characteristics and ecological or natural characteristics. It further notes that "in reality clear distinctions between [these quality definitions] can often not be made and that boundaries between categories become blurred," and that "several SFSCs actively create 'hybrids' of different quality attributes" (ibid., p. 402). Thus, although many have viewed SFSCs simply as the structure or mechanisms of exchange, how we differentiate among them depends also on the underlying attributes that the arrangements are attempting to valorize. Given the imaginary for the LRFM and the definition of LRFSs proposed here, it appears that a more accurate observation is that LRFSs are made up of a particular type of SFSC — a hybridized SFSC—which accounts not just for the structure of the arrangements but also the quality attributes they intend to convey.

This more careful approach is an important distinction, particularly when considering why the SFSC concept is so powerful in relation to understanding the arrangements of the meso level. In articulating the SFSC concept, Marsden, Banks, and Bristow (2000) offer that "[a] key characteristic of short supply chains is their capacity to re-socialize or re-spatialize food, thereby allowing the consumer to make value judgements about the relative desirability of foods on the basis of their own knowledge, experience, or perceived imagery" (ibid., p. 425). In other words, the structure of the exchange mechanisms is part of the imaginary in that it enacts systemic alterity but also this structure facilitates "giving clear signals on the provenance and quality attributes of food and by constructing transparent chains in which products reach the consumer with a significant degree of value-laden information" (Renting, Marsden, and Banks 2003, p. 398) and thus the structure imbues the products that travel through it with ontological 
distinctiveness ${ }^{24}$ from conventional products. As Grando and others (2017) point out, "[t]he 'shortness' is found... in the fact that the product reaches the end consumer [laden] with specific information regarding its characteristics, place of production, and processing methods, and that this information is channeled through specific relations between producers and consumers" (ibid., p. 2). That information is the driver of meaning and thus of economic value. Thus, the ability of exchange arrangements to transmit particular information in a particular way is thus the linchpin of LRFSs. The structures of these arrangements are where the Hinrichsian Balance between embeddedness and market-orientation/instrumentalism is made.

\subsubsection{The Value of the Conceptual Framework}

In reviewing the extant literature on alternative systems of food production and distribution, it becomes clear that conflicting understandings are pervasive and that a well-defined framework for approaching these systems is needed. The framework I propose in the preceding sections achieves much in this regard by delineating both the horizontal and vertical boundaries. With these boundaries and the clearer resulting definitions, our ability to develop and implement appropriate methodological approaches is improved and we can better understand the way the various phenomena at play within the alternative foods space function. Under this framework, we first determine whether there is a distinct imaginary being advocated for by a cohesive social movement. Given that a distinct imaginary is identified at this macro level, an opportunity space is created into which agents may enter to form arrangements of exchange. The metrics of that

\footnotetext{
${ }^{24}$ In a Lancesterian sense, this information packet is a differentiating attribute of the products, but it is one that is reliant on the nature of the exchange relationships to be transmitted through the value chain.
} 
imaginary are to be evaluated at this meso level. These are used to draw the boundaries of the system and its constituent arrangements. The behaviors and preferences of the agents forming these arrangements within the transactional environment can then be examined for deeper understanding of the system's operations, failures, and capacities.

Applying this to the local foods opportunity space, to differentiate the boundaries of it from other strategic spaces in alterity thus leads us to consider LRFSs. I contend that the LRFSs' concept to date consistently suffers from poorly defined constructs, which at times draw boundaries that are both too fuzzy to be well operationalized or too narrow and thus overly restrictive. This inconsistency has frustrated efforts to examine, understand, explain, and manage important aspects of these systems. Much of the boundary setting has focused on a single dimension — geographic distance — that creates points of contention over the metric to be used and the extent of the boundaries. However, by looking to Eriksen's (2013) proposed taxonomical approach to defining "local food" and its use of three dimensions of proximity, I offer improvements over prior approaches. I note, however, that Eriksen (2013) fails to address the systems component and thus ignores the role of the structural arrangements through which such proximity is achieved, economic value is created and transmitted, and food products with attributes of alterity are conveyed. To correct for this failure, I integrate the literature on SFSCs given that it is clear that the sets of socioeconomic arrangements discussed therein are the same set underlying the LRFSs approach and seeking to achieve the alterity that underlies that opportunity space. The literature on SFSCs presents a more coherent conceptual framework, with more neatly defined boundaries, also measured under 
multidimensionality and such dimensions relying on relativistic assessments of proximity.

Moreover, this integration provides for exposition of more refined econotheoretical explanations of LRFSs - the institutions and organizations within them, the structure of transactional relationships, and the functions these structures perform in creating and transmitting economic value between agents. Much of the intellectual development behind both the local foods and SFSC concepts has been from the standpoints of sociology and political science, with economics only more recently being considered. Although sociopolitical explanations may suffice in understanding the motivations of social movements that underlie the establishment of alternative arrangements of exchange generally, the review of the literature presented here makes it clear that economics-based explanations are needed to fully understand the how and why of the structures within these sets of arrangements and to reconcile the tension some of the literature identifies between normative and utilitarian aspects within them.

No existing work initiates the first of these developments. Here, too, the framework I develop in this chapter develops an understanding of the agents, their strategic behaviors, and their interactions, forming an understanding of how the systems and networks operate at the meso level. For the second, only one meaningful item can be identified: a conference paper, presented at a European Association of Agricultural Economists seminar, by Fondse, Wubben, Korstee, and Pascucci (2012), taking an organizational economics approach to SFSCs. More work at these intersections-both empirical and theoretical—is needed now that a streamlined framework has been 
identified centering these arrangements as the substance of AFS. Continued work in these areas is crucial for guiding pertinent public investments.

\subsection{The Scaling-Up Challenge}

A consistent concern with AFS, and especially with LRFSs, is the need to scale up such systems to ensure that they are durable and enact the alterity sought by the macro level (Clancy and Ruhf 2010; Mount 2012; Wittman and others 2012; Nost 2014; Clark and Inwood 2016). This concern is at the core of the Hinrichsian Balance in which the need for economic efficiencies and sustainable profit are in tension with deep embeddedness. This is an issue partly at the system (meso) level, insofar as it is a matter of the set of exchange relationships (arrangements). However, it is also in part a question of the economic agents that constitute those systems; any search for knowledge requires inquiry at the micro level, too. To address questions of scaling up LRFSs, we must understand the capacities and behaviors of agents constituting SFSCs, including any new forms established to address the specific contexts of LRFSs.

\subsubsection{Defining the Scaling-Up Challenge}

This scaling-up challenge is an initiative across many levels of policymaking and is expressed in a variety of particularized goals. These goals target scaling up in two primary ways. First, the attempt to increase the volume and value of food systems themselves, especially alternative, values-based, or source-identified food distribution channels. Policies that seek to accomplish this goal focus on building capacity within marketing channels to serve additional buyer segments—specifically those with larger total volume demands or that may pay a premium for specialized products—and to expand market access so that a greater number of firms — especially small and medium 
enterprises - can engage in alternative markets. Second are policies designed to increase the scale, scope, and value of the primary producers within these systems. This has come to be known as the agriculture of the middle (AOTM) paradigm (Lyson, Stevenson, \& Welsh 2008). This line of policy- and advocacy-based research and programming seeks to address the growing split in US food systems into the extremes of farm size, with a hollowing out of the middle of the distribution — mid-sized farms that are large enough to sustainably support family-owned ventures in rural communities.

It is important to note that objections to increasing the scale of LRFSs specifically or AFSs generally are frequently noted in the literature. Although these objections are often noted, few explicit references are included, let alone specific arguments treated. Only a handful of studies have made outright objections to scaling up. Navin (2015), for example, voices concerns from a philosophical perspective that sees AFNs—-he considers both fair trade and local foods simultaneously—as being at risk of losing the moral underpinnings that establish them in the first place under different transactional structures and scales. Navin's (2015) study consistently views AFNs as consisting of "shorter supply chains" versus corporatist enterprises, so it is not clear what models specifically Navin objects to or is concerned about.

Other objections are somewhat more stayed, objecting primarily to transactional models that "erode [AFNs'] authenticity or detract from the overarching objective" of the underlying social movement (Beckie, Kennedy, and Wittman 2012). Beckie, Kennedy, and Wittman (2012) cite three pieces I included in my own search: (1) Bloom and Hinrichs (2011), who consider a scaling-up approach using source-identified products sent through conventional marketing channels; (2) Friedmann (2007), who examines a 
case study in which a large institutional buyer - a university's dining services operations - relied on a third-party certification scheme to source locally identified products; and (3) Wittman, Beckie, and Hergesheimer (2012), who look at the potential roles of farmers' markets in a Canadian region's social economy. Works (2) and (3) concluded that those channels adequately maintained the alterity sought by the social movements, although (1) did not; it advocated for scaling-up policies that made investments in building use-specific infrastructure.

Some position Hinrichs as a contrarian of the scalability of AFSs, including LRFSs; Born and Purcell (2006) point to two of her earlier works-Hinrichs and others (1998) and Hinrichs (2000) — as examples of those who "are sensitive to the possibility that the local scale does not always result in desirable outcomes" (ibid., p. 196). Later, they also include Winter (2003) as falling into this local trap, as they term it. But this is not always the case as Mount and others (2013) include Hinrichs (2003) in many other works that "have cautioned against the reification of 'local food,' 'relocalisation,' and 'reconnection'” (ibid., p. 594). Beyond those noted here, few other references can be found delimiting exactly how and why scaling up LRFSs is objectionable; it seems that the literature captures phantom of the times ${ }^{25}$ perhaps present in the verbal discourse but not as vociferously in the written corpus. There is far more clarity in those who respond to alleviate these concerns.

Born and Purcell (2006) responded with their warning against being stuck in that local trap. As planners, for the field of planning, they write, in part:

We draw on current scale theory in political and economic geography to argue that local food systems are no more likely to be sustainable or just

\footnotetext{
${ }^{25}$ One may note that most of the literature cited here comes from around the early 2000s.
} 
than systems at other scales. The theory argues that scale is socially produced: scales (and their interrelations) are not independent entities with inherent qualities but strategies pursued by social actors with a particular agenda. It is the content of that agenda, not the scales themselves, that produces outcomes such as sustainability or justice. (ibid., p. 195)

Bekie, Kennedy, and Wittman (2012) adopt a related geographical approach to consider the role and ability to scale up LRFSs. Using the industrial or innovation clusters approach common to the field of management, they qualitatively examine how clusters of farmers' markets in the Canadian provinces of British Columbia and Alberta allow for scaling up at the level of the primary agricultural producer. "Participation in active clusters," they write, "not only fosters development of individual firms and markets, it also creates a collective competitive advantage through expanded horizontal and vertical linkages among public, private, and social economy sectors, facilitating a scaling up and out of regional food networks as a whole, while retaining the authenticity of the market experience" (ibid., p. 342). Aggestam, Fleiß, and Posch (2017) provide some quantitative evidence, in the Swedish context, that for these primary agricultural producers social networks have a statistically significant positive effect—-secondary ${ }^{26}$ to their psychographic profile in terms of attitudes - on their intention to engage in scaling up.

Mount (2012) also believes that, with appropriate measure, LRFSs can be appropriately scaled up without harming the alterity of the underlying LRFM, as noted earlier in this section. Mount admits that as LRFSs are scaled up, they may face challenges in maintaining identity and legitimacy. But he identifies processes of

\footnotetext{
${ }^{26}$ Also indicated as statistically significant was the operations current size, in hectares of production terms, although the magnitude of the effect was negligible which may be due to the continuous scale of the variable. Interesting to note, the study also included entrepreneurial networks, distinct from social networks, which had a negative sign but was not statistically significant.
} 
governance - specifically open processes "based on a 'negotiation of accommodations""-as the crucial tool for ensuring the viability of LRFSs and the continued creation and capture of the value that underlies them. Clark and Inwood (2016) examine issues of system governance using a case study on the LRFSs' planning landscape for Ohio's food policy-planning body. They specifically target the AOTM paradigm as a central goal to be achieved and conclude that to achieve movement on AOTM, one simply cannot simply scale up the primary agricultural producers in the system but rather must take an entire value-chain development approach, building capacity within the entire chain. It is notable that they write "[w]e demonstrate support for the concept of AOTM applied beyond the farm, for value chain development strategies that can transmit 'quality' via spatially proximate supply chains, and support for considering hybrid solutions, such as piggybacking for scaling up local food systems" (ibid., p. 503). Stevenson and Pirog (2008) had earlier identified the role a supply-chain approach might play in achieving AOTM strategies.

Mount and others (2013) conduct a wide-ranging qualitative study of LRFS agents, identifying the thematic challenges ${ }^{27}$ faced to scaling up. Although they note in their findings that the most pressing three or four themes were relatively consistent across agents, there were meaningful differences based on what they termed organizational "form" or "type"- which was the juridical/legal status of the organizations interviewed. In this, then, the structures that might accomplish scaling up becomes an important

\footnotetext{
${ }^{27}$ They identify (1) awareness and education; (2) collaboration (both inter- and intraregional); (3) economic viability (both income and organizational funding); (4) access (and the difficulties of balancing access and price); (5) policy (including regulation, government support, institutional procurement and systemic); (6) resources (including engagement and volunteer support); (7) supply and demand; and (8) infrastructure (both processing and distribution).
} 
question. Numerous entries — often single qualitative case studies — identifying various structures might be used to scale up LRFSs. Nost (2014) identifies subscription purchasing programs, known in the United States as community-supported agriculture. There are direct sales at farmers' markets, treated similarly to that in Beckie, Kennedy and Wittman (2012). Already noted was Friedmann's (2007) case of a partnership between an institutional buyer and third-party certifier; without a partnership, the institutional buyer may not be able to bear the costs of monitoring and transaction management necessary for the volume of purchases. Connelly (2010) presents the case of Seikatsu, a consumer-run food cooperative in Japan that has taken on the form of a network cooperative - that is, multiple independent local cooperatives under a single cooperative banner, a cooperative of cooperatives. Cooperatives generally are identified as a strategy for AOTM objectives by Gray and Stevenson (2008). Other studies in the literature, such as Cleveland and others (2014), identify the use of coordinating intermediaries known as food hubs. Many of these achieve scale by slightly extending the length of the underlying supply chains, and thus we are apt to continue our discussion reminded of the conceptual framework I developed earlier to help us understand LRFSs issues.

\subsubsection{Incorporating Arrangements That Can Scale Up LRFSs: Why Integration With the SFSCs Concept Matters}

It is notable that various collective entrepreneurial strategies have been identified as a means for increasing scale and, to a lesser extent, scope within LRFSs (Stevenson and Pirog 2008). Across different policy prescriptions, these include collective strategies such as cooperatives (Gray and Stevenson 2008), food hub organizations (Barham 2011; Matson and Thayer 2013), looser producer marketing organizations (Guptill and Welsh 
2008), multiventure community-supported agriculture/subscription services (Nost 2014), shared or community kitchens (Fridman and Lenters 2013), informal agrofood tourism collectives, also known as "trails" (Foreman and Westgren 2019; Foreman, Westgren, and Whetten 2013), and others. Collective entrepreneurial strategies for achieving scale go beyond traditional forms of collective action in agriculture - notably, cooperatives- to include additional forms of collaboration among primary producers (horizontal coordination), as well as those which achieve vertical coordination or varying forms of vertical integration. These may even include other stakeholders, such as public entities and civil society. McDermott, Corredoira, and Kruse (2009), working within an agrofood industry, show that the structures of these entrepreneurial networks - specifically certain kinds of connections - have a positive effect on the ability of primary producers to improve their performance and engage in product "upgrading."

This makes the integration of the SFSC concept in the preceding framework a crucial development in addressing the scaling-up challenge; these collective entrepreneurial strategies entail transactional arrangements that go beyond face-to-face interactions between producers and consumers. Early works, including seminal pieces such as Hinrichs (2000), imply — if not outright contend — that "local foods" are exchanged solely through arrangements directly between producers and consumers. That is, LRFSs, in these early conceptions, are only those with the face-to-face SFSCs listed in Table 2.2. However, the works by Marsden, Banks, Bristow, and Renting (2000) developing the SFSC conceptual model — and all of the work that integrates this model either explicitly within LRFSs or tacitly under AFSs focused on localization and spatialization - expands the set of arrangements that may constitute the meso level, at 
least to include those with intermediation. Recent practice in the United States has expanded the purview of direct agricultural marketing beyond farmer-to-consumer transactions to include marketing channels that fall within the SFSC's concept. This integration of the SFSCs concept is also useful because it mitigates problems of operationalization that arise if taking a solely geospatial approach to "local" and the constraints such an approach imposes on understanding econosystems. This expansion is crucial in addressing the concern for scaling up, as numerous works make it clear that intermediated approaches are a central requirement to increasing the volume and reach of local foods (King and others 2010; Martinez and others 2010).

These intermediated marketing channels have a twofold role. On the one hand, these channels target increasing the total number of producers. They have been identified as a strategy for supporting new venture formation — that is, new and beginning farmers and building sustainable markets for small-scale operations (Martinez and others 2010), as well as enabling entry and sustained involvement of mid-sized or medium primary agricultural producers in LRFSs (Mount 2012; Cleveland and others 2014; Nost 2014; Low and others 2015; Clark and Inwood 2016). On the other side of the supply chain, these intermediated channels address buyer and consumer audience needs. They can be a strategy for value creation and capture, or reduce barriers faced by high-volume buyers in the procurement of source-identified, socially embedded food products. As noted, intermediated arrangements can take several forms, including direct sales to restaurants and institutional food service, collective community-supported agriculture/subscription services, cooperatives, and food hub organizations. The literature indicates many potentially different challenges and opportunities for scaling up LRFSs posed by different 
organizational types and that they achieve scale differently or contribute to different perspectives and objectives on scalability, and thus that understanding these differences can better inform how to design policies that promote scaling up.

\subsubsection{The Meso- and the Micro-: Taking an Ecological Approach to Understand Agents and Networks That Achieve Scale in LRFSs}

Enquiry into the scaling-up challenge involves the meso level—here, LRFSs; it is about understanding the network of transactional arrangements leading to scalable opportunities. However, as the conceptual framework I develop in this chapter shows, to understand these econosystems, we must also investigate the constituent micro level; features of agents may enable or constrain the selection of strategies with differential capabilities in achieving increased scale, and thus affect the structure of the networks. Which forms of agents are better suited to a larger scale? Do certain forms of agents enter into specific exchange relationships with implications for system scale? Do new forms of agents emerge to facilitate larger scale? If so, what about them enables this?

These questions all reside at the micro level, but their answers help to understand the meso level. They inherently turn on the bundle of attributes held by agential kinds (“organizational forms"), not abstract, singular attributes across all agents. Moreover, these questions are about ecologies. This is the origin of the term econosystem, which I have coined. It is a play on the biological concept of the ecosystem using a portmanteau of "economic" and "system." In the next chapter, I explore how an ecological approach can help us understand the scaling-up challenge and identify a fundamental need in the social sciences that we must develop first to understand some of the questions that I posed. 


\subsection{Tables and Figures}

Table 2.1 Comparative Summary of Paradigm Dimensions: Conventional versus Alternative Agriculture

\begin{tabular}{|c|c|c|c|c|}
\hline \multicolumn{2}{|c|}{} & Conventional Agriculture Paradigm & & Alternative Agriculture Paradigm \\
\hline \multirow{2}{*}{} & $\mathbf{1}$ & Centralization & versus & Decentralization \\
\cline { 2 - 5 } & $\mathbf{2}$ & Dependence & versus & Independence \\
\cline { 2 - 5 } & $\mathbf{3}$ & Competition & versus & Community \\
\cline { 2 - 5 } & $\mathbf{4}$ & Domination of Nature & versus & Harmony With Nature \\
\cline { 2 - 5 } & $\mathbf{5}$ & Specialization & versus & Diversity \\
\cline { 2 - 5 } & $\mathbf{6}$ & Exploitation & versus & Restraint \\
\hline
\end{tabular}

Source: Redrawn from Beus and Dunlap 1990. 
Figure 2.1 Multilevel Relational Model of Alternative Food Mechanisms

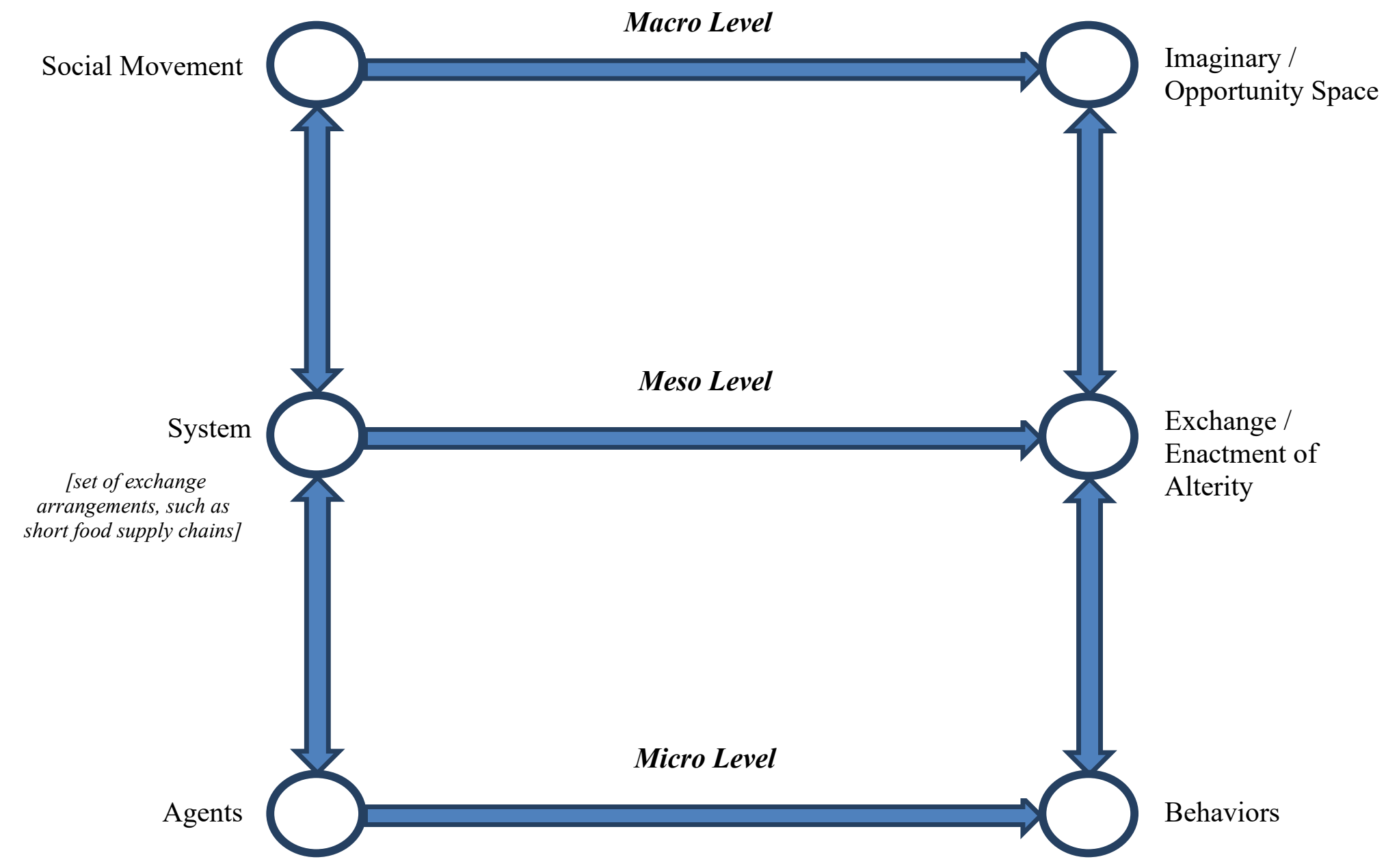


Figure 2.2 Flow Chart of Alternative Foods Space

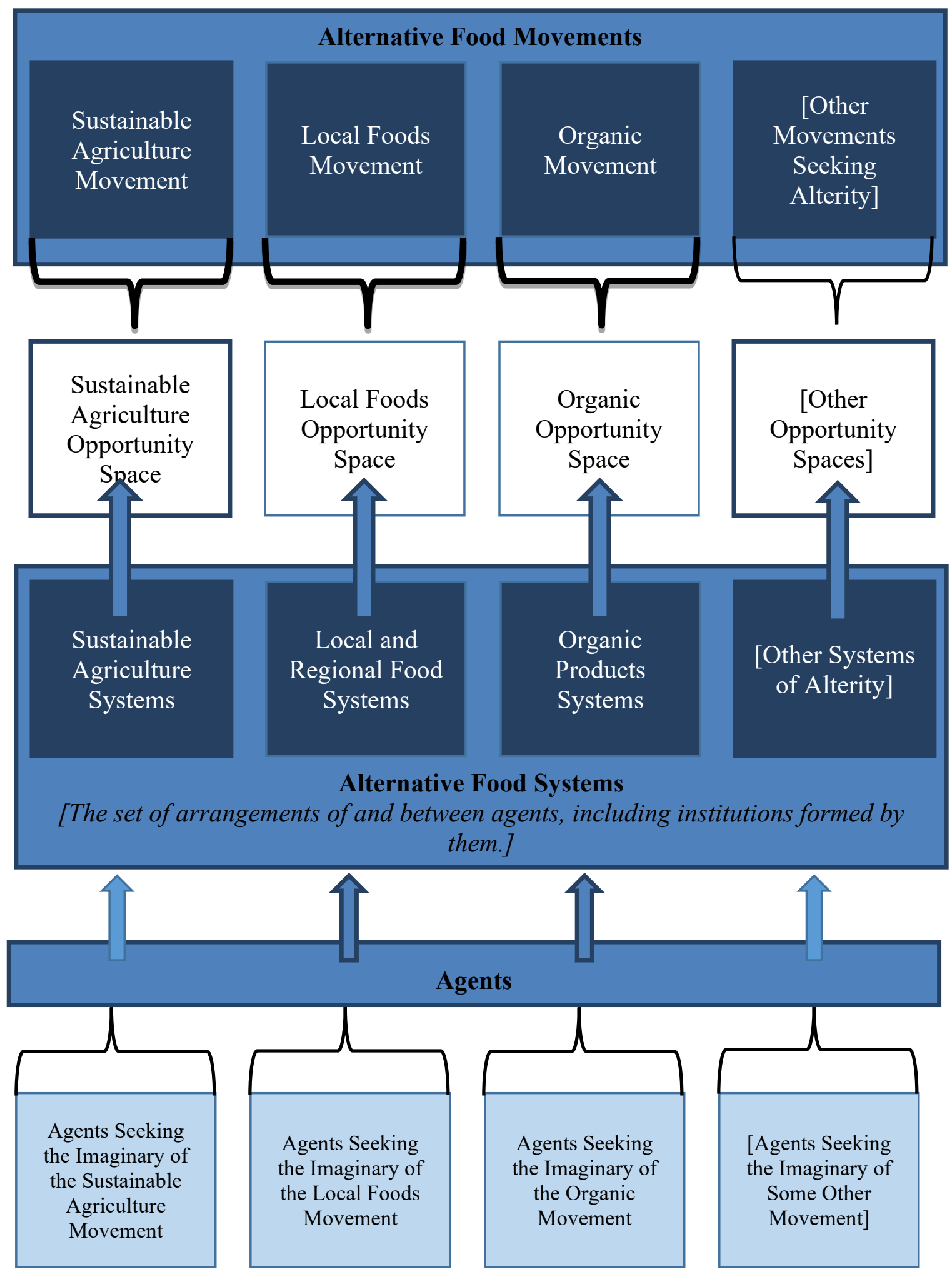

Source: Author. 
Table 2.2 Summary of SFSC Types

\begin{tabular}{|c|c|c|}
\hline SFSC Arrangement & Examples & $\begin{array}{c}\text { Common Equivalent Terms in the } \\
\text { North American Local Foods } \\
\text { Literature }\end{array}$ \\
\hline Face to face & $\begin{array}{l}\text { Farm shops; Farmers' markets; roadside stands; } \\
\text { pick-your-own; box schemes (single-farm } \\
\text { community-supported agriculture); home } \\
\text { deliveries; mail order; e-commerce }\end{array}$ & $\begin{array}{l}\text { Direct agricultural marketing channels; } \\
\text { direct to consumer }\end{array}$ \\
\hline $\begin{array}{l}\text { Relations of } \\
\text { proximity/proximate } \\
\text { SFSCs } \\
\text { (spatial or cultural) }\end{array}$ & $\begin{array}{l}\text { Farm shop "groups"; regional "hallmarks"; } \\
\text { consumer cooperatives; multifarm community- } \\
\text { supported agriculture; thematic routes (that is, } \\
\text { "trails"); special events and fairs; "local" shops, } \\
\text { restaurants, and touristical enterprises; } \\
\text { "dedicated" retailers; institutional food service }\end{array}$ & $\begin{array}{l}\text { Intermediated marketing channels; direct } \\
\text { to retail; direct to institution; direct to } \\
\text { intermediary }\end{array}$ \\
\hline Extended SFSC & $\begin{array}{l}\text { Certification labels; production codes; } \\
\text { reputation effects }\end{array}$ & $\begin{array}{l}\text { Typically considered excluded from } \\
\text { LRFSs }\end{array}$ \\
\hline
\end{tabular}

Source: Marsden, Banks, Bristow, and Renting 2000.

Note: LRFS = local and regional food system; SFSC = short food supply chain. 


\section{CHAPTER 3}

ECOLOGIES AND ORGANIZATIONAL FORMS: DEVELOPING AN ORGANIZATIONAL SPECIES CONCEPT FOR CLASSIFICATION

I established in Chapter 2 a conceptual framework of alternative food systems

(AFSs). That framework identifies that AFSs are complex and multilayered socioeconomic arrangements that arise to fulfill the desires of a social movement. Discussion developing the framework views these systems as networks or webs of agents. Thus, under the framework I develop, inquiry about AFSs centers study on the agents and their connections. In a play on words, I coin the term econosystems to describe this general notion of complex socioeconomic arrangements. It alludes to fields of inquiry in the biological sciences that study complex networks of resources and organisms in an environment, which are called ecosystems. The conceptual framework is applied to defined local and regional food systems (LRFSs) and a pressing policy question related to these LRFSs_-increasing their scale and scope-is identified. In this chapter, I expand the metaphor between biology and economics; I show how approaching economic arrangements such as ecosystems and viewing policy problems — such as the scaling-up challenge in LRFSs — as being similar to managing ecologies can be useful.

A central facet of ecological approaches is the ability to generalize systems (in biology, ecosystems) by using knowledge about kinds and their populations. In biology, those kinds are species — the basic unit of analysis for much of the field. The species concept in biology categorizes organisms into meaningfully different types. The social sciences lack a tractable, equivalent concept. Having some means of systematically categorizing agents into meaningful kinds is necessary for understanding the phenomena within econosystems and using that knowledge to address questions that stakeholders within them—such a s policymakers-pose. Therefore, in this chapter, I develop a 
conceptual approach to classification to define meaningful kinds of organizations; I term this the organizational species concept (OSC). In developing this approach, I address ontological questions on social objects, such as organizations, that are pertinent to classification. I also provide an overview of the fundamental theories of classification in organizational sciences and discuss key developments in organizational classification. With this and other material from the organizational sciences literature, I propose a framework for the OSC. In the chapters that follow, I offer a methodological approach to apply the OSC for practical classification of organizational populations. In Chapter 4, I use the developments I make in this chapter to classify food hub organizations (FHOs) in the United States. In Chapter 5, I use the resulting classification to investigate the functional roles of different food hub species in LRFSs and their implications for scaling up.

\subsection{Ecological Allegory in the Social Sciences: Understanding Econosystems}

Since the 1970s, allegory to biological theories, concepts, and models has been used in a variety of social science fields which study economic systems and the agents within them. These include research streams in the evolution and population ecology of organizations, the use of niche constructs in strategy, predator-prey models of rivalry, ecosystem approaches to resource use, and more. (For examples, see works such as Hannan and Freeman 1977; Freeman and Hannan 1983; Hannan and Freeman 1984; Carroll 1984; Carroll 1985; Carroll and Hannan 2000; Dobrev, Kim, and Carroll 2002; Wezel and Witteloostuijn 2003 and 2006; and Parker 2010.) In the sections that follow, I discuss several of these in detail. Ecological approaches to understanding and managing economic systems have increasingly been discussed, especially as a response to the failures of the neoclassical school and as a means of proliferating institutional 
arrangements that are more resilient to shocks (Wallace 2015). As that author points out in the introduction to his book:

Recent advances in evolutionary and ecosystem theory applied to economic structure and process may permit construction of both new economic theory and new tools for data analysis that can help in the design of more robust economic institutions. This may result in less frequent and less disruptive transitions, and enable the design of culturally specific systems less affected by those that do occur. (p. $x$ )

In other words, the many failures economists have encountered in modeling the complex systems that are markets can be addressed, in part, by using theory and methods that make allegory to developments in biological sciences.

I first ground this discussion in a basic understanding of the biological concepts being ported into the work of social scientists. A brief background of these concepts can help to understand how an ecological approach to LRFSs can address the scaling-up challenge. However, it also highlights why the central development I offer in this work is key to approaching this challenge from an econological perspective. Therefore, in this section, I introduce the topics of ecology and ecosystems, explore how ecological models can help to understand systems, and identify the role of kinds within ecological approaches.

\subsubsection{What Is Ecology? What Are Ecosystems?}

As Likens (1922) points out, ecology is "the scientific study of the processes influencing the distribution and abundance of organisms, the interaction among organisms, and the interactions between organisms and the transformation and flux of energy and matter" (Lawton, 1994, p. 367). Replacing the word organisms with social scientific objects such as organizations or agents in the Likens quote shows how appealing it is to use ecological allegories to understand phenomena in socioeconomic networks. At its core, 
ecology encompasses understanding the "fundamental processes" that operate in biological networks (Ernest and Brown 2001, p. 2119). That work further identifies the following salient facts of the fundamental processes:

4. Resources are required for growth, maintenance, and reproduction of individuals;

5. Because some of these resources are limited, species compete for them; and

6. No two coexisting species have identical requirements for resources and other environmental conditions (Ernest and Brown 2001, p. 2119).

Thus, ecology is the study of how interactions in a network of unique organismal types use scarce resources to create the energy needed to thrive and how changes in resource abundance affect these interactions. These networks "depend on the maintenance of multiple processes across space and time" in these shifting environmental conditions (Mouillot and others 2013).

Noting two distinct subdisciplines in the ecological sciences - community ecology and ecosystem ecology—Ernest and Brown (2001) write:

While both ecosystem and community ecology study how exchanges of energy and materials among organisms affect the structure and dynamics of ecological systems, the units of study, the nature of the important interactions, and even the fundamental currencies of these subdisciplines have traditionally been different. While community ecology typically focuses on interspecific interactions that affect the population dynamics of species, ecosystem ecology typically studies exchanges of energy and materials among broad functional groups (Ernest and Brown, 2001, p. 2118; emphasis added).

I consider both approaches under a single umbrella as part of the study of econosystems to follow the practice in social science entries studying two facets simultaneously. Even biological sciences scholars do not unanimously agree that the two fields should be treated separately. As Lawton (1994) points out, speaking of the biological concepts, "[despite often treating them as entirely distinct] populations and communities live in, and are part of, ecosystems" (ibid., p. 367). He further notes, referencing a piece he 
coauthored the same year with Jones, that "[i]f we wish ecology to progress, and to develop a more integrated understanding of nature, we have no choice but to break down the barriers that currently divide population and ecosystem science" (Lawton, 1994, p. 367). In developing this discussion, I reviewed numerous empirical works from biological ecologists following Lawton's (1994) assertion that show these two subfields as increasingly integrated — even if at times they are treated as distinct—in biology.

The two fields study properties at different levels, although both levels can be investigated simultaneously. The properties of ecosystems - that is, properties at the meso level, to draw upon the framework of Chapter 2 - such as species richness (biodiversity), total population, biomass, and energy use. Likewise, there are properties at the level of the organismal kind - such as physical and behavioral traits, interactive responses, functional roles, energy requirements, and population dynamics. Thus, the science of ecology investigates the relationship of the properties between the two levels, empirically testing theories of how changes in one realm cause changes in another.

\subsubsection{The Role of Kinds in Ecologies: The Need to Classify}

Studies on ecology abound with the word species; as the basic unit of analysis in most of macro-biology, species is pivotal as the nodes in the networks-or webs-of biological life. Ernest and Brown (2001) note, "[t]here is a long history in ecology of attempting to model ecosystem properties as the outcome of species interactions" (ibid., p. 2118). Ecologists study the roles of these populations of natural, biological kinds in ecosystems and communities. Lawton (1994) identifies many of the functions of these groupings - formed under the biological species concept—in ecosystems, writing that "[a]sking 'What do species do in ecosystems?' is [...] a question to which there are many answers; not as many answers as there are species, but too many to summarise sensibly in 
one paper" (ibid., p. 367). He identifies the most pressing function of biological kinds in ecology: examining the relationship between species' richness (that is, biodiversity) and transformation and flux of energy and matter (processes that occur in an ecosystem). Investigations of this relationship in the life sciences are varied, but a number of examples show how central are biological kinds — that is, species — in the field of ecology. Lawton (1994) presents results of a number of experiments in a controlled environment that show that the actions of some given species—or some given interaction of two or more species - can modify, maintain, or create entire habitats and thus act as ecological engineers. Ernest and Brown (2001) investigate theories on the mechanisms of equilibriums in ecosystems, noting that as environmental conditions-and thereby resource availability - fluctuate, changes in populations (abundance) of key species occurs to maintain relative homeostasis (equilibrium in resource consumption and availability) of the ecosystem. This is a matter of "compensatory population dynamics [within] interacting species“ (p. 2118) groups versus changes in the number and types of kinds in the ecosystem. "There is a large literature on the stabilizing effect of species interactions on ecosystem processes," they write, continuing that "[m]ost of these studies use models of interacting populations of species to examine potential effects on ecosystems (for example, Austin and Cook 1974, O’Neill and Giddings 1979, Post and Pimm 1983, Watson and Lovelock 1983, Loreau 1996, Hughes and Roughgarden 1998, Ives et al. 1999)" (ibid., p. 2118).

Other ecological biologists consider the effect of the mere presence of certain species in an ecosystem. Kelty (2006), for example, focuses on the effect of diversifying monoculture forestry stands to include other species. Here, the interaction with species 
newly added to this controlled forest cropping environment increases productivity of the original monoculture kind in the cropland. Mixtures of several biological kinds (species) are investigated to show the multiplicative interactions effects on biomass production and economic viability of the cropping firms. Others examine the effects of rare or minor species - those with relatively low abundance - showing that the mere presence of these kinds in the ecosystem - regardless of their relative population sizes - affects the performance of the entire system and the outcomes of other kinds.

Mouillot and others (2013) consider the first of those, what they call rare species, which are defined not only by their low abundance but also by their functional uniqueness. In other words, the traits of the kind are important. The authors point to evidence from ecological investigations that shows that

rare species can contribute significantly to long-term and large-scale ecosystem functioning, eventually providing ecological insurance in variable environments where species abundances vary in time. Indeed, rare species may perform functions complementary to those delivered by other, even closely related, species as a result of their distinct functional traits. In turn, those rare species may increase the functional diversity of local communities, sustain ecosystem functioning, and provide functional traits able to support the main ecosystem processes under future environmental conditions (ibid., p. 2; original citations omitted).

Similarly, Boeken and Shachak (2006), using controlled experiments, investigate how the presence of minor species supports dominant species in an ecosystem. Here, the minority status is in part about abundance but also about the average productivity and energy of each species in the system. Boeken and Shachak's (2006) work makes a direct link between processes at the level of the community and those at the level of the ecosystem. "The complementarity hypothesis suggests that minor species may be important due to their involvement in resource acquisition," they write, continuing that it "implies that an assemblage with dominant and minor species utilizes resources more 
efficiently than a single or a few dominant species (Walker and others 1999; Schwartz and others 2000; Loreau and Hector 2001)" (ibid., p. 119).

These examples highlight the way that kinds operate in an ecosystem, where all entities behave in relation to the species unit- the population of a species, changes of that population over time, the traits of members of a species, how these traits complement or take advantage of the traits of other species, the way that a species creates or alters attributes of the environment, and the effects of two or more species interacting on resource use and availability. These categories are not only the unit of analysis; they also set up much of the theoretical development in ecology. Thus, any ecological approach pivots on the ability to classify organisms into distinct, consistent groupings of like kind.

\subsubsection{Understanding Networks of Agents Using Ecological Allegory}

The similarities between the questions of interest to ecologists and those of interest to economists are eerie. Social scientists seek to understand, for example, how two transacting agents can create and capture value, just as ecologists investigate how two interacting organisms can create and consume energy. Economists study the effects of resource scarcity on behavior of economic agents, and ecologists ponder questions of how limited resources in a given environment create changes in organismal populations. Not every issue of concern in one field or another translates so cleanly; the allegorical reference is useful but at times tenuous. Social scientists must make adaptations to concepts developed by colleagues in biology in order to use those concepts to model and understand how social systems function.

As I initially noted, some of this work has already begun, with many ecological metaphors in the social sciences. Economics has lagged behind life sciences in modeling the complexity of systems and in accounting for multiple, simultaneous interactions. 
However, as I laid out in Section 3.1.2, ecological models use as their core unit of analysis the biological species. Thus, for an ecological approach to work, and to examine the interactions that drive the processes of the system, a basic unit of analysis is needed that identifies agential populations by their common, difference-making trait. Social scientists need to classify cases of agents into populations with distinct characteristics that play distinct functional roles in the system; we need to be able to identify agential kinds.

\subsection{A Philosophical Note: The Social Ontology of Organizations \\ Ontology has ramifications, and ontological mistakes lead to scientific mistakes.}

-Brian Epstein, The Ant Trap (2015, p. 41)

To develop an approach to classifying agential kinds, we should first consider the nature of what is being categorized. Categorization is an epistemic process that attempts to understand ontological questions. In classifying firms and organizations, we look to the realm of social ontology, which considers the nature of entities that exist of and by human interactions. These social objects are phenomena comprising facts on the anthropocentric world (that is, social facts) and the natural one. In this section, I develop the first part of a philosophical model for classifying social objects, such as firms into organizational forms. That model holds as its foundation these different sorts of facts. It integrates the work of three major philosophers in the realms of social ontology and scientific classification. Figure 3.1 depicts the final model.

In the subsequent discussion, I use a distinction important in philosophical discourse: kinds versus instances (or types and tokens). In the antitrust analyses cited by 
Cattani, Porac, and Thomas (2017), what relevant product attributes do we use to set the boundaries of a kind so that the instances (products) are interrelated by their cross-price elasticities at a level that is meaningful for legal remedy? Schoolchildren drink milk and carbonated beverages, but what relevant attributes of milk should cause an antitrust lawyer to include dairy processors in a finding of anticompetitive behaviors among soft drink manufacturers? Defining kinds for social science work requires having some justification for the boundary conditions we set. Convenience and convention are not justification. In work on organizational forms, the membership of a particular kind (category) is the instances (for example, cases or firms) that individually have the attributes used to define the kind.

\subsubsection{The Firm: Economic Perspectives on the Organization and Organizational Forms}

As the foundations of much of modern economic thought, the hegemonic neoclassical school ignores the nature and functions of the firm, viewing it as a black box - meaning that resources go in, products and services come out, and price interactions are the economist's main focus. In such a black box, various assumptions idealize the world to model the economy — or, more specifically, markets and the agents within them —as a frictionless, perfect mechanism. In response to the failings presented by this black-box approach, a "quiet revolution"—as Harold Demsetz called it in a 1997 commentary at the American Economic Association meetings- - has emerged to explain what is inside the abyss. This has led to numerous theories of the firm (Kantarelis, 2017), each covering a distinct rationale or perspective for how and why firms arise. At their core, these various approaches are related to Coase's observations that the differential in costs between specialized management of certain resources internally and purchasing 
them from the spot market leads to firms. In his note to the American Economic Association, Demsetz highlights that structural features of the firm-lines of decisionmaking authority for command and control of resources, extent of vertical integration through productive chains, diffusion or concentration of residual claims, specialized resources held interior to the firm, and so forth - are central to this theoretical revolution.

What is more, most of these conceptions imbue "the firm" with some sense of collectivity, often framed as "organizations." There is some predominant set of social entities we call firms that are social collectives commonly known as organizations that manage resources for productive ends. As Stiglitz (1991) points out in a symposium paper on organizations and economics, "[m] ost production in modern economics occurs within organizations, and this production is regulated only to a limited extent by prices" (ibid., p. 15). To use inclusive language, in this work the terms firm, collective, and organization are used interchangeably. The cases of these entities-each individual organization - are "social objects," and these social objects are central to economists" work on understanding how resources are allocated, transformed, and consumed. To unpack the black box, the field has extended itself beyond the how and the why of firms, asking questions about "the what." What roles do firms play in markets? Do different kinds of firms play different roles? What are the boundaries between kinds? Each of these may lead to yet more questions for economists and others in social sciences disciplines. Underlying these, however, is a more fundamental question of classification: what objects go in what groupings?

Economists want to classify firms to understand and predict agential behaviors (for firms, especially in resource allocation decisions), productive or consumptive 
capacities, efficiency, tradeoffs, and the connections with other types of agents that create value chains and markets—or their role in econosystems. Consider one example of how classification is important to these social scientific questions: the phenomenon of competition in a rival market. Cattani, Porac, and Thomas (2017) consider the history and prospects of categorization of firms for the purposes of studying that phenomenon. They discuss the need for categorization (of firms and products) to establish the boundaries of competitor groups in order to study rivalry behaviors and outcomes. The problem of classification for the purposes of studying competition is observed in a variety of subfields such as economic sociology (Uzzi 1997; Fligstein 2001; White 2002), organizational ecology (Podolny and Stuart 1995; Carroll and Swaminathan 2000; Hannan, Pólos, and Carroll 2007), industrial organization economics (Caves and Porter 1977; Tirole 1988; Berry, Levinsohn, and Pakes 2004) and organizational identity (Albert and Whetten 1985; Ashforth and Mael 1989; Corley and others 2006; Navis and Glynn 2010).

Across the social science disciplines related to organizations-economics, management, sociology, psychology, and others - the question of categorization falls chiefly under the guise of studies on organizational form. However, the body of literature that develops conceptual and methodological approaches of organizational form is small and is treated in greater detail in the sections that follow. More common are studies that use the term as a means of drawing comparison without carefully defining organizational form, let alone building a systematic procedure for delineating between forms. It has often been used as a rhetorical device without developing of classificatory philosophy or method that is detailed or carefully described. 
Of the literature that treats the theory and method, early works have focused on allegories to conceptual frameworks in the biological sciences, where groupings are formed in hierarchical orderings such that membership has predictive capabilities at some level, as well as explains origins or relations among kinds (Carper and Snizek 1980; McKelvey 1982; Rich 1992; Bailey 1994; McCarthy 1995, 2005). At the core is an allusion to the biological species as the basic unit of analysis for categorization and prediction. This is the foundation for what has come to be called organizational systematics, among other terms. In recent years, most of the work on classification and the determination of organizational form boundaries originates from two interrelated subfields: organizational evolution ${ }^{28}$ and organizational population ecology. ${ }^{29}$ Predominate among this is Hannan, Carroll, and Pólos' (2007) magnum opus, Logics of Organization Theory: Audiences, Codes, and Ecologies. Although the various entries in this and the extended literature enables systematically classifying firms, they also present problems, two of which are most concerning:

1. Many of the approaches eschew what philosophers of social ontology and metaphysics assert about social objects in general (Searle 2010; Epstein 2015), and social groups in particular (Epstein 2019); and,

2. Many do not adhere to what philosophers of science say about statistical inference; by not drawing classificatory boundaries around some common variance or validating them based on post hoc/a posteriori criterion, we may have only statistical artifacts.

\footnotetext{
${ }^{28}$ Organizational evolutionary studies attempt to understand how new organizational forms are created.

${ }^{29}$ Organizational population ecology studies seek to understand how populations of a given form interact with the economic environment and how different organizational forms create production-consumption networks through which they engage in economic activity.
} 
I address the second problem partly in this chapter and partly in the next, with a method for using the framework of classifying agential kinds as established in this chapter. In developing that framework, I address the first problem.

\subsubsection{Organizations as Social Objects}

In the body of literature on organizational classification, little exists on the treatment of the social ontology of organizations. Organizations are social objects, yet authors such as McKelvey treat them as biological animals, whereas those such as Hannan, Pólos, and Carroll approach them as mere vagaries of the collective imagination. The perspective adopted on what organizations are leads researchers in organizational sciences to frame their approach on how they should be classified. To develop a more holistic approach to classification, I start with a good philosophical foundation.

It is not likely controversial to state that "organizations are objects of the social world." Few would argue that they are of the natural world, whereas perhaps only slightly more might contend that they do not exist at all, merely being figments of our imagination. The general consensus is that organizations are, even if not tangible, real entities in social space. The metaphysical problem, in relation to social sciences generally, and organizational classification especially, is in properly identifying the constitutive elements. Epstein (2015) in his book The Ant Trap: Rebuilding the Foundations of the Social Sciences notes that the received view of many - that "[a] group of people is constituted by people, no more, no less" (ibid., p. 10)-is deceptive in its simplicity. Treatment of social entities, like organizations, requires more detailed consideration of philosophical elements which properly incorporates ontological complexity; as Epstein (2015) continues "[e]ven to understand the nature of simple social groups, we need to take the metaphysics seriously" (ibid., p. 10). 
Epstein develops his model in the context of social groups but as an exemplar of social objects; he views his model as "a general framework for social metaphysics" (ibid., p. 10). Thus, I approach Epstein's model from the viewpoint of social objects generally. The social phenomena he includes in his Part 1 discussion are incredibly diverse; among them include the US Supreme Court, the US Senate, faculty committees, money, andcritically—-firms (such as Starbucks). It is clear then, that, organizations and firms are social objects subject to Epstein's model generally, even if Epstein does not consider them social groups. In a review of The Ant Trap, Swindler (2016) points out that "by Epstein's lights, a group is 'a thing constituted by and only by individual people'” ( $\mathrm{p}$. 133). The US Senate is a group, as is the elderly, but money is not a group, and, less obviously, corporations and universities are not either, given that "none of these consists solely of people" (ibid., p. 105, emphasis added). The last seven words are emphasized for later; for now, I note that just because organizations are not groups does not mean they are not objects.

\subsubsection{An Integrative Approach to Social Ontology}

The first part of Epstein's (2015) study develops a model that integrates the two predominant approaches in social ontology: (a) ontological individualism, also commonly referred to as methodological individualism, inherent in the oversimplified statement summarizing the received view about social groups, and (2) the standard model of social ontology, which sees social objects as "projections of our attitudes or agreements onto the nonsocial world. ${ }^{30}$ Epstein posits that neither ontological individualism nor the standard

\footnotetext{
${ }^{30}$ The description of the standard model is the basis upon which Hannan, Pólos, and Carroll's (2007) classificatory work stands. In various writings, the authors claim that their approach simply integrates three others, one of which is a "feature-based" approach. However, the vehicle through which these features are incorporated and boundaries drawn around organizational form is still the perception of audiences.
} 
model leads to constitutive rules that are entirely sufficient for understanding social objects. The model turns on the facts about a social object, crucially those that ground and those that anchor. Grounding facts provide the conditions of the case, whereas anchoring facts set up or put in place those conditions through frame principles. I do not exhaustively treat Epstein's model here; the précis of his book (Epstein 2016) is a useful summary.

Two elements of Epstein's work in The Ant Trap are particularly relevant. First, integrating the two views of social ontology is a crucial development in light of the various theories of the firm. Some of these theories—-such as Cyert and March's behavioral theory, Alchain and Demsetz's team theory, and principal agent-based theories such as those of Baumol, Spence and Zeckhauser, and others-hinge on ontological individualism's view that social objects are composed solely of people. Others - for example, Coase's transaction costs approach, Grossman, Hart, and Moore's contracts approach, and, possibly, the resource-based view from researchers such as Wernerfelt, Prahalad, Hamel, and Barney—rest squarely on the standard model's assignment of status to nonhuman objects. ${ }^{31}$ Still others—notably Williamson's approach — view the firm from both perspectives, as comprising human and nonhuman components, reflecting a returns to the emphasis made in Swindler's words, that organizations do not consist solely of people.

This leads to the second important element. Epstein references Searle's notion of brute and institutional facts. In reading Epstein's model, both are available to form the

\footnotetext{
${ }^{31}$ One of the issues here is that Epstein notes it is an assignment to physical objects, but several of these are arguably social constructs, such as contracts and prices.
} 
constellation of grounds and anchors that merge to constitute social facts. ${ }^{32}$ Nonsocial facts affect how social objects are constituted through these various modalities. Thus, through Epstein's model, we can allow social objects to have relations to facts about both the social and physical worlds. The incorporation of Searle's brute and institutional facts is important to my purposes (1) because it fits the practice in economics of understanding firms, in part, based on their control and use of physical resources and natural, technical relationships; and (2) because Searle (1995) and Searle (2010) denote important attributes of the two sorts of facts that address some of the concerns that arise in the organizational form literature. Specifically, brute facts - those about the elements of the physical/natural world - are objective ontologically and epistemically. Institutional facts are ontologically subjective but epistemically objective. This is so despite their being intentionality relative and thus mind dependent. ${ }^{33}$

This is a crucial assertion given Searle's notation that we can understand ontologically subjective entities — such as social objects — through epistemically objective truths. In addition, Searle (2010) notes that collective recognition, agreement, or acceptance is not required of all institutional facts, contrary to Epstein's (2015) understanding. To refine the point, Searle (2010) writes that "[...] for example, the existence of a recession in the economy can be an epistemically objective fact even though it is unknown to the participants in the economic transactions" (ibid., p. 21). This implies that institutional facts and the social phenomena they underlie can and do exist

\footnotetext{
32“"As a rough guide, we can take a social fact to be a fact that corresponds to a proposition that has any social entity as a constituent. It might have social objects as constituents, or it might have social properties as constituents, or both" (Epstein, 2015, p. 67).

${ }^{33}$ Which he distinguishes from those that are purely mental.
} 
without their being the product of cognitive processes of observers or participants (that is, audiences). Thus, any classificatory approach to social objects, such as the identification of organizational forms, should allow for the existence of kinds irrespective of whether the other agents in the system or members of the cases belong to a kind set understand the case as such.

\subsection{Organizational Systematics: Classification in the Social Sciences}

For many reasons, scholars of organizations classify individuals, groups, phenomena, physical objects, and social facts, including the desire to understand and predict behaviors and decisions and to determine the what these social structures mean for the physical world. At the limit, there is no other way to comprehend and study an infinitely complex physical world overlaid with an infinitely complex social world. Problems of classification in the natural and physical sciences that have vexed scholars since the ancient philosophers-What is a species? What is the boundary of a given species? What distinguishes a statue from the bronze from which it is constructed?-have equally challenged social scientists. The domains of management and economics focus on the study of social objects—markets, firms, teams, agents, and so forth—using socially constructed categories of observation, classification, and analysis. Although the methods of classification may differ between the natural sciences and the social sciences, the intents of the research are the same. As in the natural sciences, the ability to classify human groups, social objects, social facts, and social processes permits inference, explanation, generalization, and prediction about these social entities (Boyd 1999; Ereshefsky and Reydon 2015).

The literatures in the domains of organizational scholarship that use categorization for the study of these social entities are vast, although they may share 
some concepts and constructs. In a recent study, Cattani, Porac, and Thomas (2017) consider the history and prospects of categorization of firms for the purposes of studying one important phenomenon: competition. They discuss the need for categorization (of firms and products) to establish the boundaries of competitor groups in order to study rivalry behaviors and outcomes. The problem of classification for the purposes of studying competition is observed in economic sociology (Uzzi 1997; Fligstein 2001; White 2002), organizational ecology (Podolny and Stuart 1995; Carroll and Swaminathan 2000; Hannon, Pólos, and Carroll 2007), industrial organization economics (Caves and Porter 1977; Tirole 1988; Berry, Levinsohn, and Pakes 2004) and the study of organizational identity (Albert and Whetten 1985; Ashforth and Mael 1989; Corley and others 2006; Navis and Glynn 2010). Questions on categories across (and within) these research traditions vary widely, as do the methods and outcomes of the categorization. I subsequently discuss that this is natural; I later consider these categories as natural kinds.

I turn first to questions on how categories and categorization add value to research. In our daily lives, we constantly take advantage of product categories. Imagine a grocery store without individual product categories (for example, breakfast cereals, fresh meats, canned vegetables) or a video-streaming service that does not sort dramas from comedies, animation from martial arts. To meaningfully order our lives, we categorize experiences, people we meet, objects, and ideas with cognitive tools to create mental maps of our world.

Similar to social objects made up of human decision makers, the firms that economists study are also categorizers. We, as observers of these research subjects, classify them — into types, groups, clusters, markets, industries, and other social 
categories. In their analysis of the research traditions for categorization, Cattani, Porac, and Thomas (2017) compare the lenses of industrial organization economics, strategic group analyses, and organization theory, noting their strengths and weaknesses from a methodological perspective. Although their third lens—organization theory-is overtly a social constructionist approach, the other two lenses are trained also on social constructions — often built by analysts without much attention to the cognitive processes of their subjects, the firms, and humans within and connected to them. In preparing antitrust cases, how are the boundaries established for markets so that concentration ratios and Herfindahl-Hirschman indexes can be calculated? What must be considered as a substitute product for both strategic group analysis and antitrust?

This quandary can be extended to other types of organizational research. A notable example is organizational identity research and the question of organizational legitimacy (Navis and Glynn 2010). At a basic level, the quintessential identity question of "Who are we?" implies both "Who are we not?" and "Who else is like us?" Given that "who" intersects with "what," the categorizing the focal organization and its kind becomes a question both for the social actors and for organization scholars. If the shared identity is defined by common attributes across cases that characterize a kind or form, then we are squarely in the realm of categorization. This is most clear when seeking legitimacy requirements or essential identity attributes for classification.

\subsubsection{Fundamentals of Classification Theory in the Social Sciences}

Before developing more detailed conceptual models for categorizing organizations, it is useful to first identify the fundamental world views on classification in 
the social sciences. Five central works ${ }^{34}$ discuss classificatory approaches for the organizational sciences. Two of these-Rich (1992) and Bailey (1994)—provide general reviews of the philosophical and theoretical approaches to classification, whereas McKelvey (1982), McCarthy (2005), and Hannan, ${ }^{35}$ Pólos, and Carroll (2007) propose distinct frameworks. Each of these clarifies that classification has many routes, each with very different theoretical and epistemological underpinnings.

McKelvey (1982) and Rich (1992) identify these routes as "theories of classification," both drawing on Mayr's (1969) work in the biological sciences. McKelvey (1982) notes that each such theory draws upon some distinct principles of enquiry, whereas Rich (1992) summarizes the procedures commonly used for classification, and the underlying theoretical basis and assumptions, for each in its Table 2 ("A Typology of Organizational Typologies"). McCarthy (2005) provides the general thrust of the difference between the two central routes, writing:

[...] the social sciences have two general approaches to classification: empirical and theoretical. The principal difference between the two social science approaches is the stage at which a theory of differences is proposed and evidence then sought to validate the theory (Warriner 1984, Rich 1992, Dotty \& Glick 1994). Theoretical classifications in the social sciences begin by developing a theory of differences that result in a classification of organizational types [...]. Only when the classification has been proposed, is a decision made as to where an entity belongs in the classification. On the other hand, with the empirical approach, social science classifications begin by gathering data about the entities under study. [...] the overall aim is to use data to construct the classification, instead of [using the data to] support [the classification]... (ibid.; p. 274)

However, the different branches are worth examining in finer detail.

\footnotetext{
${ }^{34}$ We identify seminal works, but note the many ancillary works preceding and following each of these.

${ }^{35}$ It is interesting that in the series of work with Carroll and Pólos, Hannan appears to have a rapid aboutface on the need for classification in organizational evolution and wider organizational population ecology.
} 
The first of the theories of classification drawn from Mayr (1969) is essentialism, "often termed the classical approach," which pivots upon the reductive principle of enquiry (McKelvey 1982). Under essentialism, categorization involves reducing objects to their "essence," which leads to artificial groupings, on the basis of relatively few characteristics that receive a priori weighting at the hands of the evaluator. These groupings are monothetic, ${ }^{36}$ "formed by rigid and successive logical divisions so that the possession of a unique set of features is both sufficient and necessary for membership in the group" (ibid.; p. 38, reproducing Sneath and Sokal 1973). An essentialist approach to classification produces (in a technical sense) typologies, which as Bailey (1994) crucially points out are conceptual classifications, not empirical ones. Thus, typologies are deductivist, and methodological approaches can include the formation of mental models ${ }^{37}$ on the basis of literature reviews, lived experience, or qualitative investigation. Statistical applications as methods tend to be confirmatory or supportive, but the evaluator has preselected the variables upon which to draw group distinctions on the basis of qualitative conceptualization.

In contrast with this theoretical approach is empiricism, which holds that groupings are naturally occurring and thus that the human cognitive activity of classification should mimic this structure as closely as possible. The empirical theory of classification takes a phenetic ${ }^{38}$ approach to the formation of groupings, using numerical

\footnotetext{
36 "Monothetic [groupings] are [those] containing cases that are all identical on all variables or dimensions being measured" (Bailey, 1994, p. 7).

${ }^{37}$ Rich (1992) calls these "commonsense" models, under a "traditional" procedure of classification, noting that they "[fail] to define [the] contents of assigned organizational groups" (ibid., p. 760).

${ }^{38}$ A "phenotype" is "[t]he observable attributes of a particular organism or organization resulting from the interaction of the genotype and the environment." (McKelvey, 1982, p. 459).
} 
methods of sorting and weighting all observed characteristics equally (that is, without a priori weighting). Enquiry in empiricism is based on the antiprinciple, where scientists avoid accepting and being guided by any theories or hypotheses about classes or groups - even whether any groupings exist. In such an approach, the evaluator records data for individual cases (for example, organizations) and forms groupings on the basis of the overall similarity of cases to one another across all variables. Thus, empirical classifications are a posteriori and polythetic, producing what is properly referred to as a numerical or phenetic taxonomy.

Although application of statistical methods may be of aid in essentialist classification, for empiricism they are the basis for classification; or, as Rich (1992) puts it: "[o]rganizational classes emerge from the empirical procedures used to sort organizational features on the basis of similarity or contrast" (ibid., p. 760). Chapter 12 of McKelvey (1982) provides a detailed description of common numerical methods, but it is clear from this, as well as Bailey's (1994) more cursory discussion, that cluster analysis is the predominant tool, supplanted with additional multivariate and graphical numeric methods. Empiricism's phenetic approach should be considered with caution; as McCarthy (2005) points out, "phenetics discounts any theory that might explain differences, such as the theory of evolution for biological organisms and the theory of electron structures for chemical elements. It simply contends that the best measure of relatedness is overall similarity" (ibid., p. 278). In stark contrast with essentialist, empiricism can be effectively theory-free. One link between essentialism and empiricism exists: for the most part, these theories produce classificatory schemes that are synchronic. 
A diachronic theory of classification is that of phyletics, which McKelvey (1982) denotes is primarily composed of the "subtheories" evolutionism and cladism. Of the three ${ }^{39}$ primary theories I highlight here, phyletics "is the only theory that attempts to explain the origins of groupings, as well as classify them" (ibid.; p. 50, emphasis added). Thus, phyletics kills two birds with one stone: classification and explanation. The outcomes of this approach are also taxonomies, but unlike those produced under empiricist approaches where the only affinity that binds group members is their ahistorical numerical similarity, any given taxa (that is, class) in a phyletic approach has affinity among the members of the class that is common to some historical progenitor. The two subtheories in phyletics disagree on the nature of this affinity. Evolutionism favors patristic affinity, "the number of similar characters that members of a given [taxa] derived from a common ancestor," whereas cladism favors cladistic affinity, or the "recency of descent from a common ancestor, without taking into account the number of shared characters they might have" (ibid., p. 51, emphasis added).

Two principles of enquiry fall under these theories of classification: the rational principle (individual cases are explained by looking to the wider system in which they reside) and the holistic principle (allowance for two-way causal flows and extreme caution to avoid the fallacy of composition). Phylletics, at least as applied in organizational science, largely takes a mixed-methods approach, with historical and qualitative analysis being used to establish the evolutionary path of taxa and cases within them, and the multivariate statistical methods used in empiricism's phenetic approach to

\footnotetext{
${ }^{39}$ McKelvey (1982) and Rich (1992) note a fourth, nominalism, which in the McKelvey work is discussed in a cursory single page entry and dismissed handily because it quickly fell out of favor in the life and natural sciences and philosophy.
} 
(recursively) corroborate classes and hierarchies. McCarthy (2005) puts phyletics under an empirical banner. McKelvey (1982) and Bailey (1994) note that evolutionism's patristic affinity has links to phenetics, ${ }^{40}$ of which some appear to be ontological and others practical. Noting these links, McKelvey (1982) positions evolutionism as a kind of bridge or halfway point between the totally oppositional phenetic view of empiricism and (purely) phyletic view of cladism.

\subsubsection{Organizational Systematics: A Taxonomic Approach to Classification}

This link is central to the primary development that McKelvey (1982) offers: organizational classification should be tailored to an approach that hybridizes empiricism and evolutionism — in other words, a combined phyletic-phenetic approach. This approach is termed organizational systematics (McKelvey 1982; Rich 1992) McKelvey (1982) posits, to summarize the more detailed arguments there, that such a combined approach (a) makes for better science and (2) accounts for the mutually intelligible and mutually beneficial history between phenetic and phyletic approaches in the biological sciences. These two components validate and corroborate or constructively question each other.

Rich (1992) notes that a taxonomic approach (that is, one where groupings are polythetic) accounts for the observation attributed to the work of Hempel (1965) in the philosophy of science that "in scientific research the objects under study resist tidy pigeonholing and often exhibit traits that are gradated rather than discrete" (Rich, 1992, p. 764). Moreover, consider the assertion by Knox (1998) that "[w] hen isomorphic with

\footnotetext{
${ }^{40}$ This leads Bailey (1994) to contend that evolutionism is "strictly speaking, synchronic." However, discussion in McKelvey (1982) and even Bailey's (1994) own contextualization cast doubt on the strength of such an argument.
} 
nature, [classificatory schemes] are useful for organizing and manipulating our knowledge" (ibid., p. 1, emphasis added), which favors a natural classification approach for scientific understanding over an artificial one. McCarthy (2005) astutely ${ }^{41}$ asserts: “Both McKelvey [(1982)]'s and Rich [(1992)]'s propositions support the underlying tenet $[\ldots]$ that there is a need for organizational science to develop jointly a broad theory on how organizational diversity is generated, along with a system of organizational classification that coincides with this theory" (ibid., p. 286).

Behling (1980) succinctly summarizes these objections raised to a taxonomic approach in organizational sciences:

1. Uniqueness. Each organization, group, and person differs to some degree from all others; the development of precise general laws in organizational behavior and organization theory is thus impossible.

2. Instability. The phenomena of interest to researchers in organizational behavior and organization theory are transitory. Not only do the "facts" of social events change with time, but the "laws" governing them change as well. Natural science research is poorly equipped to capture these fleeting phenomena.

3. Sensitivity. Unlike chemical compounds and other entities of interest to natural science re searchers, the people who make up organizations, and thus organizations themselves, may behave differently if they become aware of researchers' hypotheses about them.

4. Lack of Realism. Manipulating and controlling variables in organizational research changes the phenomena under study. Researchers thus cannot generalize from their studies because the phenomena observed inevitably differ from their real-world counterparts.

5. Epistemological Differences. Although understanding cause and effect through natural science research is an appropriate way of "knowing" about physical phenomena, a different kind of "knowledge" not tapped by this approach is more important in organizational behavior and organization theory (ibid., pp. 484-485).

\footnotetext{
${ }^{41}$ However astute the observation, McCarthy (2005) advocates for and develop a cladistic approach to organizational classification, which conflicts with McKelvey's (1982) explicit preference for evolutionism.
} 
Whatmore and others (1982), in the explicit context of classifying primary agricultural producers, raises "three $[\ldots]$ weaknesses [that] undermine the explanatory value of taxonomic typologies" (ibid., p. 25): (1) objects of study are reduced to their separate observable features; (2) the "regularity thesis of causation which underlies the positivist research method"; and (3) production of classificatory schemes that are "static and ahistorical." However, in carefully reading Whatmore and others (1982) and the primary works it references as examples of these concerns it levels against "taxonomic typologies," I conclude that those examples are not taxonomies under the frameworks discussed in McKelvey (1982), Rich (1992), Bailey (1994), and McCarthy (2006). Moreover, Behling (1980) reports the list replicated earlier as background of a work centered on providing argument in favor of a "natural science model of research in organizational behavior and organization theory." (p. 483) I have been unable to identify any serious attack within far more contemporary organizational science literature reigniting concerns over such approaches or making a clear argument in favor of only an essentialist approach. Thus, early arguments against taxonomics are unconvincing.

It is important to consider how researchers to date have proposed how to proceed with work in organizational systematics (which both classifies and explains origins). The three aforementioned works —McKelvey (1982), McCarthy (2005), and Hannan, Pólos, and Carroll (2007) ${ }^{42}$-present the most prominent frameworks in the field. Two general approaches have been identified by McCarthy (2005). The first begins with theoretical classification to which organizational entities are subsequently assigned. The second

\footnotetext{
${ }^{42}$ It is interesting that in the series of work with Carroll and Pólos, Hannan changes his opinion on the need for classification in organizational evolution and wider organizational population ecology.
} 
begins with data about the properties of the entities, from which types are constructed. In the former, kinds are defined a priori — that is kinds are the theoretical design. This McCarthy (2005) calls "theoretical," whereas Rich (1992) terms it "theoretic" and includes McKelvey (1982)'s "phyletics." In the latter, data on properties are the basis for constructing the classification design. The latter is termed "empirical," "numerical," and “empiricism," respectively.

McKelvey (1982)'s hybridized approach to classification recursively melds ${ }^{43}$ the numerical (phenetics) of the second with phyletics, specifically with the patristic affinity ${ }^{44}$ of his "Evolutionism." Under the hybrized approach, we define the basic taxonomic unit through atheoretical investigation of organizational morphology (that is, based on observable, objective features). The resulting taxa (groupings or kinds) are later placed into hierarchies depending on phylogeny. Through this recursive systematics we can test hypotheses about the mechanisms that underlie the emergence of new organizational forms. Diverging from McKelvey (1982), McCarthy (2005) advocates for a cladistic ${ }^{45}$ approach to forming hierarchies and explaining origins. Both McKelvey (1982) and McCarthy (2005) emphasize the metaphysical properties that imply organizations are real entities, or "individuals," and that groupings are naturally occurring. It is notable that both McKelvey (1982) and McCarthy (2005) place the atheoretical discovery of the kinds as the starting point; ordering the kinds at the level of the basic taxonomic unit—whether phylogenetically or cladistically—into hierarchies is the imposition of a theory of differences and thus a testable hypothesis.

\footnotetext{
${ }^{43}$ Rich (1992) also argues for integrating the approaches.

${ }^{44}$ Looks to the number of similar characters that members of a taxa derived from a common ancestor.

${ }^{45}$ Favors the recency of descent from a common ancestor, without accounting for number of shared characters.
} 
The third framework central to the literature departs wildly from this stance. Pólos, Hannan, and Carroll (1998) and Hannan, Pólos, and Carroll (2007)develop a social constructivist view of organizational categorization, relying on "audiences" to delimit organizational kinds and eschew the notion that organizations are entities (that is, that they are ontologically distinct, with forms that exist outside the minds of those who perceive them). This approach to defining organizational form, the authors assert, integrates three others: a "social boundaries-based" view taken from Hannan and Freeman (1986); a "network-based" view rooted in DiMaggio (1986); and a "featurebased" view that is similar to the phenetic approach of McKelvey. ${ }^{46}$ The difference is that here the features are identified by the audiences.

Their approach hinges on "fuzzy" boundaries that allows for multiply realizable membership where any given organization may be a partial member of a class. Their approach depends on a process of legitimation to define the boundaries of organizational form-the same process that is an underlying evolutionary mechanism. This connection is made clear in later works, including that of Bogaert, Boone, and Carroll (2010), which expressly refers to the 2007 work as a "revised theory of organizational evolution to interpret and re-specify the legitimation part of the density dependence model" (ibid., p. 115). In this, then, the trio's classificatory scheme not only wholeheartedly adopts the Standard Model of social ontology for organizations, it also departs from the two-stage process and incorporates an evolutionary mechanism — which should be tested

\footnotetext{
${ }^{46}$ However, the work of McKelvey is not cited there. Across the central works in the line of research by Hannan, Carroll, and Pólos, only a single reference to any of McKelvey's work can be found: it is relegated to a single footnote of a working paper, dismissing it offhand as "lacking empirical bite" and providing no further meaningful discussion (see footnote 1 on p. 1 of Hannan, Pólos, and Carroll 2004).
} 
independently for many different kinds - into the process of identifying organizational forms (that is, it puts evolution into the equation when establishing the boundaries between kinds of the basic taxonomic unit). This is problematic.

Hannan, Pólos, and Carroll's (2004) account is not merely an exercise in postmodern social construction. It meaningfully contributes by identifying that although biological allegories (such as viewing the organization as an organism and studying populations thereof) are useful, because organizations are social objects we cannot ignore aspects of intentionality and social construction which are not accounted for in biological theories as they are not at play among natural objects. However, this intentionality is more aptly considered as a mechanism of change (that is, evolutionary process) in population structures, not a method for delimiting populations of interest on which to test evolutionary theories. For the latter, we must ground the analysis in the observed - not perceived - morphology of the entities, treating them as distinctly ontological. Moreover, that body of work can be reframed as a meaningful way to identify niches as it more closely mimics the way life sciences consider ecologies.

In the arguments that favor a taxonomic approach to organizational classification, a central starting point is clearly the performance of phenetic work; numerical taxonomies are, in contrast with the history in the biological sciences, the most accessible avenue to establishing a foothold for an organizational systematics. What has drawn me to this conclusion, beyond the more philosophically oriented discussion in the literature noted earlier? The answer lies in an [otherwise] fruitless search for classificatory examples in the literature. Despite McKelvey's contention from 40 years ago, much of the classificatory work on organizations - most particularly that within agrofood 
systems - has remained mired in an essentialist approach. This has led to a never-ending patchwork of contradictory typologies that, although useful in their idiosyncratic contexts, fail to provide meaningful understanding and explanation of general matters important to organizational science, economics, and sociology. Moreover, these essentialist classificatory schemes fail to reflect the natural or organic occurrence and development of social organisms, approaching them instead with the evaluator's preconceived notions. Even in what may first seem to be robust attempts at numerical classification, these preconceptions problematize classifications and their use in scientific enquiry. To address these failures, I develop a new framework to classify organizations into distinct forms.

\subsection{Developing a Framework for Organizational Classification to Delimit Forms}

Why is the lack of a coherent classificatory system problematic? Romanelli (1991) identifies one of the central issues, writing that under an early camp in the literature on organizational evolution, where classification is a critical component, “...it is not easy to know how findings [about evolutionary mechanisms] may generalize to other forms of organization" (ibid., p. 83) without a generally accepted and consistent method for identifying distinct populations of organizations. Moreover, many of the classificatory approaches do not adhere to what philosophers of science say about statistical inference; by not drawing classificatory boundaries around some common variance or validating them on the basis of ad hoc and a posteriori criterion, we may have only statistical artifacts. An additional fault is including the same or correlated processes identified as evolutionary mechanisms as classificatory elements - a problem similar to selection on the dependent variable. Many of the extant approaches also eschew what 
philosophers of social ontology and metaphysics assert about social objects in general (Searle 2010; Epstein, 2015), and collectives and groups in particular ${ }^{47}$ (Epstein 2019).

In addition, other issues arise - two epistemological and one historical. Without consistent classificatory guidelines, researchers struggle to test proposed mechanisms of evolution or identify the relative weight that different evolutionary mechanisms may play in given circumstances. We also struggle to understand the ecologies of organizational populations, unable to differentiate what roles specific organizational forms play within the econosystem, how changes in the population of a form may affect resource decisions and outcomes, or how evolution of new forms can affect other organizations within a space. Last, the development of organizational evolution without a proper theory of classification is an ahistorical allegory to how evolutionary theory came to be in the biological sciences; biological theories of change started from works attempting to classify and order the world around us, not the other way around.

I noted previously as an example of the importance of classification in the social sciences the ongoing research stream on competition. One question posed in this literature to address when considering innovation and its consequences for competition is whether the categorization frame is focused on the attributes of the products or the attributes of the firms. Is product competition about the products per se and the attributes that make them substitutes for buyers in the market? Or is competition about the behaviors of the members of the strategic group that produce the competing goods? One might respond by saying that framing the distinction this way is entirely facile; it ignores

\footnotetext{
${ }^{47}$ Several key works in organizational evolution imply that organizations themselves do not exist ontologically (that is, outside the mind of an audience), let alone explicit denounciations that natural kinds do so.
} 
the subtle links between firms and their products and services. I agree. But the implication follows that we must pay close attention to how we define innovation, how we populate rivalry groups before and after significant innovation events, and how the mental models of the agents in the rivalry groups and their customers are accommodated by the categories we construct.

This specific case — of competition and rivalry groups of firms—illustrates the general problems we face in the categorization of social objects generally. Here we return to my earlier promise of an integrated philosophical model for classification of social objects such as organizations. In Section 3.2, I began this development. In section 3.3, I detoured from it to see how classification of organizations has developed thus far. That background is important now as I close the loop and complete the philosophical model; the historical approaches in organizational systematics provide context to the subsequent philosophical understanding. The philosophical model concludes at the end of subsection 3.41, where it is presented in Figure 3.1. It addresses the failures of those early works in organizational classification I note earlier. The rest of the subsections here (3.4.2 and 3.4.3) extend the philosophical model with theoretical considerations on its final stage and then propose in a holistic sense the formalized organizational species concept.

\subsubsection{Patterns and Properties: Integrating Boyd, Searle, and Epstein}

The model begins with the social ontology of organizations (section 3.2) and thus is rooted in the facts—-both brute/natural and institutional—about those social objects. To get us to classifications of these objects, two developments in philosophy speak to the problems of categories. The first development is the way that Richard Boyd (1991) constructs natural kinds: groups of things that are clustered meaningfully by science, so that inquiry about the kind can lead to explanation or induction. Boyd is a philosopher of 
science and addresses categorization — defining kinds — through the lens of the problems surrounding the definition of species. Biological species cannot be about shared attributes alone, particularly morphological structures, lest we misplace instances in our taxonomy (hierarchy) of kinds. His account, developed in a long discourse with other philosophers of science, has become the received view (Ereshefsky and Reydon 2015) or the consensus view (Samuels and Ferreria 2010) of philosophers of science because "it better captures the kinds of the biological and social sciences than other theories of kinds" (Ereshefsky and Reydon 2015, p. 970). In studying organizations it is useful that Boyd requires a proper study of natural kinds in social science to accommodate scientific practice in determining the boundaries of a social kind. To wit, "Natural kinds are features, not of the world outside our practice, but of the ways in which that practice engages with the rest of the world" (Boyd 2003, p. 538). Social psychologists may not define organizational kinds in the same manner as do industrial organization economists, strategic management scholars in the tradition of contingency theory, or cultural sociologists. This is not bad science, but good science requires making clear how to apply Boyd's desiderata.

The central feature of Boyd's account of natural kinds for the biological and social sciences is homeostatic property clusters (HPCs). A number of properties or attributes of the members of the kind repeat across instances and over time. These clustered attributes are at the core of the categorization process and behave in a homeostatic manner - they remain consistent. Put another way, not only are the properties immutable, but so too is their relative co-incidence. This is important to the biological sciences. Species and taxa must have some consistency and constancy over 
time so that membership is meaningful, as new instances are incorporated and extinctions are observed. That is, there is epistemic value to the clustered attributes over time for inference, explanation, prediction, or generalization (Boyd 1999; Ereshefsky and Reydon 2015). Fans of organizational identity research will see the original Albert and Whetten (1985) virtues of central, distinctive, and enduring in the definition of HPC or the less controversial "persistence" or "continuity" as substitutes for endurance (Corley and others 2006). By using organizational identity as a scientific basis for describing natural kinds of organizations for the epistemic functions of explanation or generalization and a fact about these kinds is that some of the central or distinctive properties change over time, then the needs for categorizing and change must be accommodated in our approach. Otherwise, there is no basis for defining any identity that is shared across instances and types; every organizational identity is individuated, and we are left with instances without kinds.

In addition to HPCs and the epistemic functions they contribute to, Boyd's account includes the need to include "underlying mechanisms or processes that tend to maintain the presence of the properties" (Boyd 1999, p.143) and hold the clustered properties together. That is, returning to organizational identity, what causes the defining cluster of attributes to endure and to maintain distinctiveness in relation to adjacent categories. We do not require causal mechanisms to describe and define categories in our borrowing of Boyd's account of natural kinds. A number of other forms may hold the clusters together and there may be other ways to identify the regularities of our social kinds. This argument evokes a second recent development in philosophy: Brian Epstein's analyses of social groups (Epstein 2015, 2017). 
Epstein approaches the categorization of social groups, including organizations and firms, that are formed purposefully from the perspective of metaphysics. That is, he is interested in the properties of human groups. In his book, The Ant Trap: Rebuilding the Foundations of the Social Sciences (2015), Epstein admonishes us about the problems of methodological individualism. First, he offers the distinction between ontological individualism (the belief that group properties supervene upon the properties of individual members of the group) and explanatory individualism — the belief that social explanations (that is, of group behaviors) can or must be explained by the actions of the individuals that constitute the group. Methodological individualism as it is invoked in economics and management confounds these two. Epstein notes that philosophers of science question explanatory individualism and we should not feel comfortable using this approach in social science. Moreover, we should be equally uncomfortable with ontological individualism, because an account of the group members' attributes misses too much about the nature of the group and how it behaves. If a group is a social actor and fodder for categorization, we must consider other social facts of the group beyond its membership. Epstein models these social facts by how they are grounded and anchored. The identity of the organization may be partially grounded not only by its constituents but also by habits, conventions, precedents, and other social facts of the group. These may be anchored by written by-laws, statutes of incorporation, or other artifacts of the identification process including those that are conferred by external stakeholders.

Epstein (2017) carries this argument further. He profiles human groups-ranging from street buskers to faculty committees to corporations to social classes-using four broad classification criteria: (1) the construction profile, (2) the extra essentials profile, 
(3) the anchor profile, and (4) the accidental profile. This is a system of defining classes of social groups as natural kinds, without causal mechanisms. Criteria (1), the construction profile, shows the requirements for coming into existence and for continuation, the nature of constitution (membership), and formal identity requirements for any instance of an organization to be counted as a particular kind. Sometimes these requirements are consistent across the profile through time. We are certainly interested to the extent that an organizational instance that we are studying enters a particular category (as a new entrant into a given industry category) or exits (by no longer producing the appropriate class of goods). For some organizations, they may be fleeting or intermittent members of a class and the construction profile details this. The extra essentials profile contains those important properties that are not about the membership (constitution) per se of the group. They are the abilities (capabilities?), rights, norms, and obligations of the group and - important for Epstein, Boyd, and us — and of the members of the group. We can define behavioral and structural properties of a kind/category of organization by observing and using the properties of the member firms. Important for Epstein and for porting the logic of HPC to our studies for industry or market category membership, this is part and parcel of scientific accommodation.

The attributes that describe startup firms in our research may be known only a posteriori, unless there is a profoundly useful theoretical basis for category construction. We may posit some of the properties that cluster for startups a priori, but we may comprehend more of them as we make specific inquiries about the category members. Hence, we add these properties to the category definition. Criterion (3) is important for our studies of organizations. What anchors the central and distinctive properties? Are 
they anchored in conventions, shared dominant logics, statutes of incorporation, or other laws and regulations? Or must we augment Epstein's ontological approach with Boyd's causal approach by considering technological or social mechanisms that explain the continued homeostasis. Criterion (4) according to Epstein is the same as Boyd's a posteriori definitions; we find the attributes in the "actual world" and what anchors them. Metaphysics meets method.

Now we have a micro-foundational approach to categorization of products, rivalry networks, and organizations; it is founded on the metaphysics of kinds and instances but permits the inclusion of anchoring and causal mechanisms as part of the ontological status of the categories. This should appeal to scholars of organizations who seek to model the processes of institutionalization, such as Scott's (2008) three pillars and the routines, mechanisms, indicators, and the underlying social anchors. One may use Scott's model for categorizing institutions or for categorizing organizations constrained by institutions and recognize that his tools conform to the desiderata of Boyd and Epstein.

\subsubsection{Econological Niches: Adaptations of the Niche Construct in Economics, Management, and Organizational Sciences}

In the final component of the philosophical model, represented in Figure 3.1, I hold that kinds become species only when the kinds are meaningfully distinct. This is a process of validation, which ends in a decision to accept or reject the set of kinds as a set of species. In other words, we seek to justify holding the true belief that the kinds that emerge as HPCs have, at minimum, objective epistemic value. To perform this process of validation, we need some metric or construct by which we determine that kinds are meaningful. For this, I turn to another common allegory made to the biological sciences by those in the social sciences: the niche construct. In another pithy portmanteau, I term 
the social scientific version of this construct econological niches, similar to econosystems. Let us consider some central developments from biology about the ecological niche and how it has been adapted and operationalized as it crosses the metaphorical divide into social science.

\subsubsection{Ecology: The Environmental Niche and the Population Niche}

Early biologists, such as Elton (1927) held the niche to be an "address" in the environment, waiting to be discovered or exploited by an organism. The modern view sees the niche as the manifestation of how a particular organism uses extant resources in competition with other organisms and how the organism, in turn, affects the environment. That is, the conception of the ecological niche has changed from the notion of an environmental niche to the idea of the population niche (Colwell, 1994). Elton (1927) defines the niche as environmental attributes (or also other relevant species in the food chain) that provide a population opportunity for reproductive survival. According to this view, niches can be vacant, awaiting exploitation. Hutchinson $(1957,1991)$, on the other hand, defines the niche as a population attribute described in terms of environmental factors. The fundamental niche is described by the range of environmental factors, defined by phenotypic attributes (hence, it is a population niche as opposed to environmental niche), which are required for persistence of the organism. However, because of competition with other overlapping species, the realized niche in which species persist is the hyperdimensional subspace without overlap with competitors. Figure 3.2 represents the relation between the fundamental and realized niche in a hyperspace. According to this modern conception of the niche, the exploitation of an ecological opportunity cannot be the filling in, or adaptation to, a vacant niche. Rather, 
the organisms respond to a change in phenotypic attributes and abiotic resources that exist in competitive space: a niche shift. A vacant niche is a meaningless construct.

Furthermore, in the hyperspace are multiple adjacent and overlapping fundamental niches. If the dimensions of the ecological hyperspace include gradients of moisture levels, elevation, canopy cover, nutrient levels, and so forth, then the fundamental niches would appear as in Figure 3.3. The dynamic nature of these dimensions would cause the fundamental niches to "move" in the space. As a result, organisms would vacate their realized niches as a result of environmental change and the resultant competition among organisms with overlapping fundamental niches.

Consequently, the two notions of the ecological niche imply that changes in the ecological niche are either exogenous or endogenous relative to the population of organisms. Changes in the environmental niche are exogenous. Alterations in available resources and other relevant species generate "new" niches that are successfully discovered and exploited if attributes of the target population are adaptive. On the other hand, changes in the realized population niche are endogenous. Niche change is understood as the phenotypic change of the target population in response to new selective pressures - a given organism population either adapts or is replaced by a more successful population.

\subsubsection{Niche Construction}

The evolutionary canon holds that natural selection fits organisms to the environment. Ecological constructionists have heavily criticized this one-way view discussed in the previous section. They show that adaptedness can be achieved the other way around: organisms can construct or alter environmental factors to fit their own features. The niche construction approach, first proposed by Lewontin (1985) and later 
elaborated by Odling-Smee and others (2003), holds that both natural selection and niche construction should be taken into consideration when explaining adaptation. OdlingSmee and others (2003) argue that organisms can actively modify the relationship between organismic features and environmental factors. Niche construction is the process in which this relationship is modified through the physical altering of environmental factors, or through the relocation and thus exposure to a different surrounding. Evolutionary consequences occur when the niche construction effects are persistent, thereby creating stable selective pressures.

Niche construction can be divided into four categories along two dimensions: whether the effects are inceptive or counteractive and whether the source of change is perturbation or relocation of the environment (Odling-Smee and others 2003, see Table 3.1). The inceptive dimension shows how organisms can create new niches by changing selective pressures. The counteractive dimension shows how the effects can be neutralizing. The dual process of natural selection and niche construction results in feedback dynamics that cannot be explained by natural selection alone. The following equations describe the changes of the two constructs: environment and organism (Levins and Lewontin 1985):
(1) Environment $(t)=f($ Environment, Internal Factors $)$
(2) Organism $(t)=f($ Organism, External Factors $)$

An example of how inceptive niche construction can have evolutionary consequences is the construction of spider webs. Web spiders build webs to capture insect prey, creating it when there is none and repairing it when it is torn. The persistent existence of webs results in the feedback selection of phenotypes that are adapted to live 
on the web. The Cyclosa genus constructs dummy spiders for predators, whereas the Segestria and Mallos spiders communicate by tapping the threads of the web. These recipient phenotypes would not have evolved without the construction of these local environments (Odling-Smee and others 2003). Another example of niche construction's counteractive effect is temperature regulation. Insects that cannot survive under low temperatures create nests and burrows that increase the local temperature (Odling-Smee and others 2003). The negative selective pressures of low temperatures are thus canceled out.

The most recent work on the niche permits organism-environment interactions that cover the alternatives discussed earlier, wherein the organisms may have agency to change/construct their niche, adapt through phenotypic plasticity, or to disperse into other fundamental niches. This modern view is reviewed and codified by Chase and Leibold (2003). They propose a model of interactions between organisms and their environment, including other organisms, that elaborate the formation of the realized niche and include effects of organism behavior on the environment. Competition, mutualism, and resource use is explicit.

\subsubsection{Bridging the Metaphorical Divide}

The use of the niche construct in management, especially organizational sociology, dates from the 1950s, although Popielarz and Neal (2007) point to Simmel (1922) and Park, Burgess, and McKenzie's 1925 treatise on urban environment (1925) as important precursors. Popielarz and Neal carefully review the development of the literature around social structure and population ecology. Figure 3.4 shows the explicit borrowing from the ecology literature discussed earlier in two seminal works: McPherson's 1983 ecology of affiliation and Hannan and Freeman's 1977 population 
ecology of organizations. Although this figure ends with the two seminal works, the review discusses important additional imports from ecology in the 1990s, including the concepts of niche width, resource partitioning, and niche dynamics. The time lag between the development of new models in ecology and their adoption/adaptation in management means that we do not see yet the existence of a unified approach to organization niche theory that mimics Chase and Leibold (2003).

Perhaps the reference that all literature reviews on the organizational niche should begin with is Astley and Van de Ven (1983), who used the older conception of the niche as a "location" in the socioeconomic environment, not as a functional fit between the organizational attributes and attributes of the business environment. Thus, resource constraints and structural inertia of the extant niches drive the selection processes for well-fitting organizations and the dispersal or failure of others. In the construction of their central debates in organization theory, they rely on this version of environmental determinism to be touchstone to discuss more voluntaristic theories of firm action (that is, strategy) or collective action — the two schools highlighted by Popielarz and Neal.

Astley (1985) followed up by comparing a population ecology approach—defined as the dynamics of a single population government by isomorphism and legitimationwith a community ecology approach that explicitly combines multiple interacting populations and has a more evolutionary perspective, which may account for new "species." This view is compatible with the aptly titled The Ecological Theater and the Evolutionary Play by G. E. Hutchinson (1975), a series of public lectures in which Hutchinson considered a dynamic interaction among organisms networked in space marked by environmental resources and a variety of connections between them-the 
work of Chase and Leibold (2003). Astley's paper is entirely conceptual and lacks much of the new learning in ecology provoked by Hutchinson in 1975.

Hannan, Carroll, and Pólos (2007) revisit the niche in a formal model of the social relations between the members of an organizational form and their audience(s) and Popielarz and Neal (2007) examine it in a comprehensive review paper. Both of these papers state the need to separate the fundamental niche from the realized niche, although both see the study of the niche as occurring after the niche is realized, that is, the requisite mimetic and selection processes for defining the organizational form have been completed. Regardless, the niche is clearly a core construct related to the emergence of new forms, as entities adapt morphologies and behaviors to use resources and take advantage of opportunities, but does not specify the mechanisms of adaptation and thus evolution.

\subsubsection{The Organizational Species Concept: At the Nexus of Kinds and Niches}

Section 3.4.1 conveys a micro-foundational approach to category formation, founded on the metaphysics of instances and kinds but which permits the inclusion of anchoring and causal mechanisms as part of the ontological status of the categories when relevant. However, this does not ensure that the boundaries between kinds are properly chosen. We need a method to validate that proposed boundaries are meaningful, without relying on the same constructs we hope to test between the boundaries. I turn to the niche, a common allegory made in the social sciences to the biological concept. Thus, cointegrating the concept of natural kinds with niches, I conceive an organizational species as a being a natural kind of social organism, on the basis HPCs, that tends to inhabit a particular niche of the social world. Such a conception accommodates the interests of an array of social sciences that interest themselves with organizational 
forms - economics, sociology, finance, and management, to name a few-as it allows for the identification of meaningful classifications that help predict behaviors and outcomes. Such a conception accommodates the interests of an array of social sciences that interest themselves with organizational forms - economics, sociology, finance, and management, to name a few-as it allows for the identification of meaningful classifications that help predict behaviors and outcomes. In the work that follows, I focus on a niche that is rooted in the strategic orientation of a selected case: food hub organizations. However, other niches are likely worthy of future investigation.

The next logical phase is then how does one determine an organizational species? This problem is the concern of the remainder of this work. In the following section, I examine a methodological approach to classification, a form of exploratory science. A problem persistent in organizational systematics work is determining at what level is a taxon (a grouping within a hierarchy) appropriately a "species" (McKelvey 1982; Rich 1992). The answer to this question is a straightforward use of a counterfactual: if application of the methods described in this chapter fails to provide meaningful groupings and/or those groupings do not tend to inhabit a particularized niche, then the likelihood of species existing at the level of analysis is low. By extension, then, if our investigation consistently fails to indicate the presence of distinct natural kinds, then it is appropriate to move one level up, and examine whether the population under consideration is itself a potential organizational species.

\subsection{Operationalizing the Organizational Species Concept: A Methodology to Search for HPCs and Identify and Validate Kinds}

The central task in applying the organizational species concept I develop is to search among a population of organizations for patterns of "sticky" (that is, homeostatic) 
properties that lead to groupings of cases where membership in a given grouping is associated with habitation in a particular niche. This search for patterns of properties is the stuff of numerical phenetics, and we can operationalize this process by looking to the statistical methods common to this and other empiricist approaches to the identification of kinds. To accomplish this, researchers across disciplines have turned toward aptly named cluster analysis procedures — statistical algorithms and supporting analytical tools that allow for polythetic (common multiattribute) analysis of patterns. In the management and organizational sciences, for example, cluster analysis has a history of application in the research stream on strategic groups of firms (Harrigan 1985; Barney and Hoskisson 1990; Ketchen and Shook 1996) and the identification of consumer market segments (Dolnicar 2002; Morton, Anable, and Nelson 2017). However, because cluster analysis is a widely applied method, developments in its theory, practice, and implementation originate from statistics, computer science, psychology, education, biology, and anthropology. Only recently has the field of cluster analysis gained prominence; thus, comprehensive texts on updated approaches and best practices have newly come into the literature - with some still forthcoming. Unless otherwise noted, the discussion that follows is based on the voluminous contents of Henning and others' (2016) recent handbook or on the unpublished lecture materials in Steinley (2019) derived from his forthcoming text.

Cluster analysis is not one single thing - there is not one single algorithm or even a unificatory approach that one can use to identify groupings of cases based on observed properties. A number of texts offer typologies of the various approaches and their underlying algorithms, each typology different than the last. The central differences lie in 
the underlying assumptions about the mechanism that links an individual cases together; some cluster analysis approaches link cases on the basis of their distance from each other in the hyperspace (hierarchical clustering analysis), others by partitioning the hyperspace along vectors on the basis of local optima (kMeans clustering), whereas still others search for statistical distributions that match the observed data (mixture model clustering), and yet others look to the density of cases in the hyperspace to form groupings (such as the DBSCAN algorithm). It is important to identify that no matter the underlying statistical theory or concept, algorithms used in cluster analysis seek to link individual cases on the basis of their position in a multivariate hyperspace, where each measured property is a dimension of that hyperspace. Thus, the HPCs that underlie kinds are uncovered through procedures that link cases on the basis of the sum of their properties, not simply because some specific property obtains. This accommodates the discussion in previous sections denoting that a case may be an instance of a kind, even if the case does not function exactly as the other instances of that kind may.

Before continuing, consider some fundamental best practices for cluster analysis (Barney \& Hoskissen 1990; Ketchen \& Shook 1996). Critics have pointed out several problems - issues of construct validity, external validity, and reliability — that are frequently found in studies employing these methods, such as strategic groups. Specifically, these critics claim that most researchers make critical errors in (1) not providing sufficient justification for the choice of variables, distance measures, or clustering algorithms used in their analysis; (2) not checking the overall reliability of their cluster solutions by using different clustering parameters; and (3) using inappropriate means of assessing the validity of their solutions, typically performing a 
multivariate analysis of variance or discriminant analysis on the variables used in the cluster analysis itself, rather than on a set of variables reserved for the purpose of validation.

\subsubsection{Adopting a Hierarchical Clustering Analysis Approach}

I have briefly noted several of the different clustering procedures commonly applied in the search for kinds. It is important to note several other pertinent aspects, beyond just the underlying theory of how cases are linked into groupings. Especially salient are the questions of how cases relate to the groupings and how groupings relate to one another. In hierarchical cluster analysis (HCA), one of the earliest approaches developed, cases are ordered — based on the chosen linking rule — into nested sets, such that membership in a "child" set imposes membership in the "parent" set. Thus, HCA procedures produce a tree of nested sets, called a dendrogram, with each case being, at the lowest level, in a set of one-itself the only member. In this approach, every case is assigned to a set in any given partitioning (that is, cutting of the tree to form groupings).

Under most algorithms for the vectorization approach, of which kMeans is an example, every case is assigned to a grouping (based on its position within a "cell" of the hyperspace created by the vectors) just as with HCA. To run these algorithms, however, the research must specify the number of vectors (and thus the number of cells and therefore number of clusters) for the optimization procedures to run. This means we either must know how many clusters exist, or we must run the algorithm multiple times with different numbers specified and find an optimal solution given some predefined criterion. However, unlike in HCA, the groupings are unrelated and are wholly independent. 
In the approaches exemplified by HCA, kMeans, and DBSCAN groupings are in closed sets, sometimes called "hard clusters," where membership is discrete—you are either in, or you are out. So, too, do many of the algorithms in these approaches require that partitioning of the sets be strict, with each case being a member of only one set at any given clustering solution. This is not the case for distribution-based clustering using mixture model approaches. In these algorithms, each case is assigned a probability of membership for each set. However, like with kMeans, this requires the researcher to specify the number of sets that (are believed) to exist. Doing so tells the algorithm the number of distributions to search for, and the model runs iteratively to find the parameters of that given number of distributions that best fit the observed data. Although distribution-based approaches such as mixture models have many attractive features, in addition to the need to specify in advance the number of sets, they also impose strong assumptions about the underlying data that may not be realistic for observed natural data.

Of final note is that for many of the density-based approaches, such as DBSCAN, cases may be omitted from partitions, left unassigned to groups and treated as outliers. In this regard, such algorithms perform "partial" clustering, compared with the "complete" clustering of the other approaches noted here. This is generally because the underlying algorithms search for differentials in the density of the hyperspace, treating sparsely populated regions effectively as null sets. This is problematic when we expect that otherwise meaningfully different kinds may overlap in the hyperspace (that is, the kinds share some properties but are sufficiently different in others so as to be distinct). Furthermore, some of the most common algorithms under this approach do not provide information in their outputs on the relationships between grouped sets. 
Thus, among the most common statistical approaches to cluster analysis, HCA is the only one that provides direct relationships between grouped sets. It also ensures, through its agglomerative approach, that all cases are assigned to sets (that is, clustering is complete). Unlike most of the other approaches, it does not require specification of the number of sets to be extracted as kinds in advance; in fact, HCA is typically advocated for as the best approach taken in situations where there are little or no empirical indications on the number of kinds that may exist, making it the gold standard for the initial stages of exploratory science in classification. This is the position most of organizational systematics is in, and most assuredly where we are at in wanting to identify organizational species to understand the ecologies of LRFSs. Therefore, I adopt HCA as the preferred approach to analyzing data of organizational populations in LRFSs to search for HPCs and identify kinds. In Chapter 6, I discuss possible extensions into other clustering approaches to be completed in the future. In the sections that follow, I outline procedures for performing HCA and validating the kinds it produces as organizational species. These procedures thus provide a pathway that fully operationalizes the OSC.

\subsubsection{Procedures for Identifying Kinds and Validating Them as Species}

In the three sections that follow, I describe those procedures. In general, these can be deemed as stages, or phases, of a statistical methodology for identifying kinds and validating them as species. However, the search for patterns among data on a sample of instances (here, cases of firms) that form HPCs is not necessarily a linear process. It is an interative one, and thus within any given "stage" of analysis, components of prior stages may be resurrected. This is especially the case for statistical tools, applied in subsequent phases of the discovery process to different variable elements. At times, the researcher is 
warranted to backtrack entirely to an earlier stage on the basis of observations and learning that occurs in a latter one. This is, in some sense, a process of Bayesian updating, albeit not necessarily in a statistical sense. The three phases in this process of updating are as follows: (1) determining what variables are to be used as morphological properties (features) of the cases and which are proxies for the strategic orientations of kinds; (2) searching for HPCs using cluster analysis techniques and best practices, including determination of the most appropriate clustering approach and partitioning solution; and (3) validating the kinds that result from the search process as species, or rejecting the partitioning set and restarting or concluding that the sample may represent a distinct population in its entirety, to be validated as a species within a wider population (that is, an expanded data set).

\subsubsection{Selection and Refinement of Variable Sets}

The first task in the investigatory procedures is to select the sets of variables that represents the properties of interest for use in identifying HPCs and as representative of the organizational niches in which species may reside. That is, we must select two sets: (a) morphological variables of the organizations within which we search for patterns to group cases and (b) outcome variables that act as proxies for niches within market space which can be used for validation of a final postulated clustering solution (FPCS) as a set of organizational species. The selection of variables, which contain information about the properties of cases, for use in clustering and validation procedures - most especially the former-occurs iteratively throughout the exploratory investigation of kinds, but is the sole focus of its preliminary stage.

In this stage, the initial set of variables is selected by the researcher using three elements: (1) theoretical considerations and the meaning of variables as properties of the 
firms; (2) data visualization techniques which allow the researcher to observe the "behavior" of data and different transformations thereof; and (3) statistical methods that provide quantitative information and inferences about the relationships among variables. One additional consideration must also be made when working with data on natural observations: the quality of a variable's underlying data, in terms of reliability of the construct (that is, did people understand what was being asked) and the completeness of the set across cases (that is, missing data). As regards the latter, it is important to note that in the majority of situations in cluster analysis generally, and HCA particularly, imputation of data is not an option, as it violates the underlying assumption that if clusters exist, it is because cases of that kind draw from a distribution for a given property that is unique to the kind.

To an extent, element 1 is idiosyncratic to the nature of the population being studied; the theoretical rationale for whether a property is pertinent to the morphology of the case, the niche in which the case's kind resides, or simply not pertinent at all depends on the anchoring facts about the selected population and the frame these set up. However, as noted previously, in an applied setting, our task is to search for clusters of (morphological) properties that are homeostatic and thus "sticky," to use a common economic parlance. From an economic perspective, this is akin to intermediate or fixed assets - those features of the firm that we cannot readily change within a given decision horizon. Thus, as a starting point, we should primarily (although perhaps not exclusively, given other theoretical considerations) seek those variables, observed at time $t$, which include information on firm properties that we can reasonably expect to be at or near the observed value at $t \pm 1$. 
Multiple tools exist within the available kit, which can be used for visual investigation of potential variables under element 2 . In the procedures I use, two are selected: scatterplot matrices and multidimensional scaling (MDS) plots of common space. The first of these conducts bilateral comparisons of variables, plotting the paired observations for every case with one variable on the $x$-axis and another on the $y$-axis. The plots for the $n$ combinations of variables are presented in a massive grid, much like a bivariate correlation table. Along the diagonal, histograms of each variable are depicted. The researcher examines these visualizations to see if there is evidence of separation into distinct groupings among the plots. Such a finding is a sufficient, but not necessary, indicator of likely underlying clusters among the cases. These matrices are often very large and typically are not reported out. They are useful in very early stages of variable selection.

Once a core of potential (morphological) variables is selected, the researcher conducts MDS, a form of data reduction. These procedures include, typically, running MDS at four, three, and two dimensions. In my work, I use the default settings within SPSS as these are the most commonly accepted parameters, reducing case-wise. The outcome is a set of coordinates that can be used to map cases into visual common space. Evaluation criteria of the visualizations center on the separability and density of cases into groupings within these reduced dimensions. Outputs also provide quantitative measures that can be used to evaluate the general acceptability of the dimension reduction, notably the normalized raw stress, a badness-of-fit criterion that indicates in standardized form the discrepancy between the distance of cases in Euclidean space versus their observed dissimilarity. Similar to scatterplots, MDS observations are a 
sufficient, but not necessary, indicator of underlying clusters. In addition, they can help us compare the underlying structure within a data set across two different sets of variables, and thus can help indicate through comparative visualization if a considered variable is acting as a mask to otherwise "natural" cluster structures.

Last, there are some mathematical and statistical approaches which can be used to gain additional information about the "behavior" of different variables. Two of theseprincipal component analysis and factor analysis-I do not pursue in my work here because they pose a number of concerns that cannot be overcome at the current moment. Both of those tools are additional data reduction techniques, with the first a mathematical decomposition of eigenvalues and the second based on statistical models. I do, however, include simple bivariate correlation analysis in my procedures for variable selection. One should not misconstrue the simplicity of the analysis, which relies on Pearson correlation coefficients, with a lack of meaningful information. It is quite the contrary, as the procedure allows us to engage in Element 3-type investigations to draw inferences about the likelihood of the results among the observed sample to the overall population. Here, if a pair of variables shows a high absolute value for the correlation coefficient-especially if this value is indicated as being statistically significant—-then we should view inclusion of both variables in the clustering procedures with suspicion. For example, if two variables are likely to measure the same underlying construct or causal mechanism, then inclusion of both within cluster analysis has implications for the relative weighting of that property. Results of correlation analysis also help the researcher identify potential precursors to patterns in data that do not appear in visualizations, and can inform the choice between two or more approaches to the same property of the firms (for example, 
different scales of the same variable or maintaining greater informational richness instead of reducing variables to a single composite measure).

By applying the tools for each of these three elements, we can separate out variables for use as niche proxies as well as create one or more "core sets" of morphological variables. These core sets are entered into HCA algorithms to form a set of baseline clustering models to initiate the search for HPCs and identify organizational kinds. Throughout the subsequent stages of the analysis, we may alter these core sets by dropping, adding, or substituting variables, depending on the performance of the clustering algorithms. Following the preliminary selection, this should primarily be done to accommodate informational richness by avoiding dramatic losses in total number of included cases or when new evidence indicates theoretical error or the presence of masking variables.

\subsubsection{Formation of a Final Postulated Cluster Solution}

Once one or more baseline models are established, we enter Stage 2, working toward a FPCS, which is arrived at through an iterative process ${ }^{48}$ of inductive ex post, and deductive ex ante updating between rounds of analysis. In these rounds a series of cluster solutions are computed and evaluated. The result — the FPCS—is the assignment of cases to groupings (clusters) that arises from a chosen partitioning (that is, cutting of the dendrogram) of results from a linking algorithm (that is, "method") applied to a given set of variables under given specifications for certain parameters (usually the measure of similarity and approach to variable standardization). Early rounds may test these latter

\footnotetext{
${ }^{48}$ Such a process is highly stressed by Ketchen and Shook (1996) as a critical means of enhancing the internal validity of a cluster solution.
} 
two for the sake of completeness, but theoretical and mathematical properties also may indicate the preferred specifications. In later rounds, or when certain parameters are indicated a priori, we focus on evaluating variable specifications, linkage method, and partitioning. The FPCS represents the theorized kinds upon which organizational species boundaries can be drawn if indicated in the validation stage as being associated with habitation in a specific niche.

In my analysis, the four most common HCA linking algorithms are used: (1) the single-linkage or nearest-neighbor method, (2) between-groups average linkage method, (3) the furthest-neighbor or complete-linkage methods, and (4) Ward's method. Because this is an early-stage analysis, where identifying outliers is preferred, squared Euclidean distance is selected as the sole measure of similarity (proximity in multidimensional space). In early rounds, we initially tested standardization approaches. However, empirical findings in the literature on cluster analysis indicate the a priori selection of standardizing variables to the 0-to-1 range, especially when dealing with mixed-scale data where one or more variables are dichotomous, whereas others are continuous. The once common method of standardizing over $z$-scores is now highly disfavored because of the statistical implications on variances and assumptions it imposes about underlying distributions.

Results of solutions with different parameters (here, primarily linking method) and variable sets are compared and evaluated by the researcher using both quantitative and qualitative assessment measures. Qualitatively, we inspect results for face validity using statistical information about cluster mean profiles (resulting from ANOVA analysis), visualizations — both two- and three-dimensional — of clusters mapped into 
MDS Common Space, and dendrograms and their joining-tree heights. These are relatively straightforward or have already been discussed. Quantitative tools used that are worthy of detailed discussion include the cophenetic correlation coefficient, the CalinskiHarabasz index, Akaike information criterion, Bayesian information criterion, and the adjusted Rand index.

The cophenetic correlation coefficient assesses the fit of the hierarchical structure and is conceptually similar to the raw stress measure in MDS. It calculates a correlation of the vectorized matrix of distances and similarities between cases and the vectorized cophenetic matrix - the matrix of heights in the dendrogram at which any two cases are joined. Thus, when Euclidean distance is the similarity measure used, the cophenetic correlation coefficient relates the degree to which the hierarchical nested sets recover the underlying relative positions of the cases in Euclidean space. It does not provide any guidance as to "where to cut the tree" (that is, dendrogram).

For that we turn to the Calinski-Harabasz index, the Akaike information criterion, and the Bayesian information criterion. The Calinski-Harabasz index is the gold standard among these and provides information on the internal cohesion and external isolation of clusters resulting from different partitionings. It is alternatively termed the minimum Ftest because the index has the same form of the $F$-statistic calculated in traditional ANOVA; it finds the weighted proportion of between sum-of-squares errors (external isolation) to within sum-of-squares errors (internal cohesion). The Calinski-Harabasz index is calculated for each of the $k$ partitions in a considered range-usually between 2 , the minimum number, and 12 - and the partitioning solution with the maximum value in the range is indicated as preferred. The Akaike information criterion and the Bayesian 
information criterion, on the other hand, provide measures of informational entropy. ${ }^{49}$ These measures are well discussed in the literature and offer a relative means of examining the fit of models. Both include elements that penalize complexity and prefer parsimony; thus, we would expect them to favor clustering solutions with fewer clusters.

The final quantitative tool used in these procedures - the adjusted Rand index (ARI) - allows us to assess the similarity of case assignment to groupings between two clustering solutions. It is notable that the adjusted part of the ARI comes from the fact that this measure accounts for the random chance of grouping elements together, and thus is effectively probability weighted. The components of the ARI are worth noting, as they are counts of case pairings and thus the measure accounts for all cases paired to all other cases in the data set and the agreement or dissonance of their assignment to a grouping. ARI values are also standardized, on a range of -1 to 1 , which makes the measures comparable across partitionings within the same matched pair of clustering models. Values between 0.65 and 0.79 are considered a sign of acceptable recovery between two clustering/partitioning solutions, whereas those from 0.80 to 0.89 being "good" and those above 0.90 being "excellent." Unfortunately, because ARI requires that the case set be identical for comparison, if any two clustering solutions rely on variable sets where there is even a one-case difference then it cannot be computed using standard statistical software packages.

\footnotetext{
${ }^{49}$ See Murari and others (2019) for an extended discussion on entropy and the Akaike information criterion and Bayesian information criterion measures.
} 
In each round of the Stage 2 procedures, the researcher performs the same analytical functions. Typically, these occur in the following order, although perturbations from it are not problematic in most cases:

1. Defining a variable set and clustering parameters,

2. Running the clustering algorithm(s) to formulate case assignments for each model across the specified range of partitionings,

3. Inspecting the resulting dendrogram to ascertain the shape and nature of the hierarchal structure,

4. Computing the cophenetic correlation coefficient, the Calinski-Harabasz index, the Akaike information criterion, and the Bayesian information criterion and assessing the general performance of each model run and the level of convergence on a partitioning level (that is, number of case groupings, $k$ ) both within a model and across models

5. On the basis of these results, selecting one or several partitioning solutions for each model in the round, computing the ARIs for every available pairing of selected solutions, placing them into a matrix, and evaluating the general recovery across clustering solutions

6. Conducting ANOVA on one, several, or all clustering solutions for indicated models and partitioning levels to examine face validity of morphological mean profiles and the incidence of significant $F$-statistics

7. Mapping group assignments of cases into MDS common space to ascertain the orthography of the clusters in low-dimension space

8. Determining whether there is/are one or several clustering solutions for promotion as a FPCS for validation, or else reformulating variable sets and parameters to address problems and initiating a new round of analysis for cluster formation

For item 6 , we expect more variables than not to show statistically significant differences of variances (measured by $p$ value of the $F$-statistic) across groups. Thus, what we ascertain with this ANOVA is whether there are surprising numbers of nonsignificant variables, which would indicate few differences in the patterns of HPCs between the groupings, as well as changes in patterns of statistical significance across partitioning solutions within a given model. Item 7 is only a worthwhile exercise if the normalized 
raw stress for a given variable set and at a given dimensionality is below the established threshold, indicating the reduction is generally an acceptable approximation of the multivariate hyperspace in Euclidean space. Other heuristics, including the reasoned judgment of the researcher on the basis of their understanding of the population of firms and theory, may enter this process, especially in functions that rely on qualitative assessment. It is worth advising the researcher to have conducted qualitative fieldwork to develop an understanding of the population to be classified in advance of the clustering procedures and an attempt to establish organizational species. Once item 8 is achieved and the researcher has a FPCS, we move to the third and final stage of operationalizing the OSC.

\subsubsection{Acceptance or Rejection of FPCS as a Set of Organizational Species}

The FPCS in hand is at this point still a theorized set of case groupings. Under the OSC, we must validate (or reject) the kinds identified by the FPCS using outcome variables to accept them as species — as distinct organizational forms. These outcome variables are to be post hoc and preferably somewhat uncorrelated with any single given morphological property evaluated; the point is that the pattern of HPCs should drive any distinction between the kinds for them to be species, not a spurious single property. This approach of using post hoc outcome variables for validation is a best practice identified in the cluster analysis literature. Under the OSC, the researcher is guided on the nature of the properties that should be positioned as outcomes: proxies for organizational niches, which here I operationalize as elements of the firms' strategic orientations.

This investigation is conducted through two routes: (1) determination of differences in variances and means among and between groups and (2) statistical procedures to determine whether species membership maintains statistically significant 
differences when controlling for exogenous variables. These exogenous variables too should be distinct from, and to an extent uncorrelated with, any given morphological variable used to form cluster membership. Under the first route, ANOVA allows us to investigate differences in variance across all groups, whereas independent-samples $t$ tests allow for testing the equality of variance and differences in means between any given pairs case groupings. ANOVA procedures are straightforward, conducting the statistical procedures using the cluster assignments as the categorization variable and examining the mean profiles, confidence intervals, and significant of the $F$-statistic to determine if there are meaningful inferences to be made about differences in variances across all groups. Independent-samples $t$ test procedures are more complex, as they are performed in two waves. The first wave compares each kind identified by the FPCS to the subset of all other cases in the clustering model not of that kind. This provides information on the relative departure of the kind from the other cases for each variable of interest, in terms of mean differences. The second wave compares each kind to each other kind identified by the FPCS, thus enabling analysis of group-to-group differences. Also important is that independent-samples $t$ tests provide a method for testing the ability to make inferences about comparative differences in variances (whereas ANOVA is a pooled difference) as well as for mean differences (where ANOVA provides no information about the ability to make inferences about means at all). It should be noted that independent-samples $t$ test results produce massive quantities of data, which can be difficult to report out. However, this informational richness is a boon to the researcher in terms of assessing just how different the kinds are from one another and important how certain we can be of the magnitudes of the observed differences. 
Standard regression analysis techniques are used to achieve the second route of validation analysis. In the regression models specified, the strategic orientation representing niche constructs are used as the dependent variables. These are regressed on species membership, acting as a fixed effect. Additional variables accounting for exogenous factors are then incorporated into the regression estimations, when available. These can account for environmental factors such as regions, economic climates, and other limiting factors on strategic choice sets, or they can incorporate other variables about the firm that are not homeostatic in nature but that theory indicates may have an effect on strategic choices of the firm. In my applications, I use general linear modeling with fixed effects components. The selection of regression techniques should match the situation on hand, including the nature of the outcome variables entering the left-hand side of the models and other features of the data. The generalized linear model approach I take in my procedures is simple but effective in providing basic evidence on the effect of membership in a kind on presence within a particular niche. Those whose data allow them to conduct even more robust and complex regression procedures may wish to do so, but I make no recommendations here because this is not a work focused on econometrics.

\subsubsection{Adopted Methodology, Abductive Reasoning, and Inferences}

This approach provides the researcher with a rich set of objective information that characterizes the nature of the kinds and the patterns of facts underlying theminformation with which they can form beliefs and make judgments and decisions. This is not to claim that there are only objective elements present; as with any research process, there are subjective decisions and judgments to be made by the research team. The point is that they can do so with objective justification. For example, under proposed procedures, all properties are treated with equal weight in the search for patterns. Cluster 
analysis allows for pattern recognition that includes not only binary but continuous and qualitative elements, a point that Epstein (2019) emphasizes as critical in classification of social objects (see, for example, its footnote 38 ).

Cluster analysis procedures translate these multivariate properties into a "distance" between cases, forming groupings based on the nearness of cases. The mathematical properties of the joining rules and distance measures are known and can be analyzed. Although there are numerous approaches, among them are those that form groupings based on variance among the cases such as Ward's method under HCA and kMeans, a nonhierarchical method. Other more advanced procedures seek clusters based on probability distributions (Gaussian mixture model clustering) or density parameters (DBSCAN). Furthermore, the set of partitions/groups can be characterized by statistical measures internal to the clustering solution, such as the Calinski-Harabasz index, the Akaike information criterion, the Bayesian information criterion, and others, thus characterizing the partitioning result.

The approach also compares candidate kinds, meeting Epstein's assertion that kinds be evaluated in relation to one another. In Stage 1, this occurs quantitatively through the ARI and inspection of morphological variables using ANOVA and independent-samples $t$ test tools to determine the degree of differentiation, as well as qualitatively through inspection for face validity of mean profiles and orthographies of clusters in MDS space. The Stage 3 application of ANOVA and independent-samples $t$ test procedures to outcome variables also accomplishes this by quantifying the separation of kinds within niches. This provides information on meaningful differences in terms of magnitudes and overlaps. 
These tools also provide us with information that guides our confidence in those differences actually being true through statistical significance test. Across each stage, statistical tools are used which provide a means of statistical inference via the probability of obtaining results at least as extreme as those observed, given the appropriate null hypothesis. Such tools include correlation analysis in Stage 1, the cophenetic correlation coefficient and elements of MDS (for example, normalized raw stress) in Stage 2, and the ANOVA, independent-samples $t$ tests, and regression analysis components (coefficients and model fit measures) in Stages 2 and 3. It is crucial that the last of those components - regression procedures - test whether the differences in the post hoc a posteriori features of the kinds (here, their strategic orientations) are likely to arbitrary or spurious associations, or if they are driven by membership in a candidate kind.

It is important, too, that these procedures allow for recalibration of boundaries (including number of candidate kinds) and thus form the basis for adductive reasoning process. They provide information that supports the researcher in making an inference to the best explanation - in our case, the number of candidate kinds that have distinctive patterns of HPCs associated with habitation of the proto-member (that is, the average, ideal, or representative case) for any given kind within a specific niche of the social world. In this case, differentiable organizational forms are under the OSC. If the evidence obtained through the search process contained in these methodological procedures is insufficient to make a final inference that a set of candidate kinds where $k \geq 2$ is a set of organizational forms (that is, where we cannot form a justified belief that there are differentiable kinds in the observed cases), then we may infer that the entire set of cases may be a unitary kind. To verify this, we must expand our observation of cases and 
evaluate using the same methodological procedures here to determine whether the original set forms a distinct kind in relation to those newly observed.

\subsection{Concluding Remarks: The OSC, Species, Ecologies, and Scale}

An ecological approach to economic activity views markets as interconnected networks of agents. This is not unlike the "food chain" or "food web" common in introductory biology courses. Within these food webs resources are transformed into "energy," or for the economist and businessperson "value." Different organizational forms pursue different strategies to access resources and transform them into value. This means they play different roles in econosystems, filling different niches. Their structures are likely adapted to the unique environments within these niches. Thus, we want to delimit organizational forms. To do this, I develop the OSC as a means of searching for HPC among instances (cases) of firms that are validated as distinct populations on the basis of their pursuit of a specific strategy, a proxy for the niche they inhabit. Unlike prior attempts at categorization of firms, my OSC specific does not make claims about the mechanisms of adaptation, selection, or evolution; in this I follow the guide of McKelvey, who posits that organizational forms can and should be identified first as a means of testing theories of origins - that is theories of adaptation, selection, or evolution.

The method for operationalizing the OSC to identify organizational forms (cum species) I set forth here is adapted to circumstances where we do not have a priori empirical evidence of kinds and thus must engage in the discovery process of exploratory science to investigate the presence of kinds and species within an otherwise artificially crafted population set. Proper application of the method does not mean that the starting population set must be broken down; quite the contrary is true, as the approach to 
operationalizing the OSC can end in a null result where we conclude there is not sufficient evidence to infer that distinct species exist within that wider population. In such an instance, the researcher would expand the initial population set, reapply the OSC operationalization to this wider set of cases, and validate that the initial population of interest is itself a distinct species. The method I propose, and the OSC generally, meets the dictates of McKelvey and others that the discovery of kinds be atheoretical—that kinds emerge from the data, not vice versa.

Moreover, the OSC is useful beyond a desire of fundamental scientific inquiry to test theories of organizational evolution. The concept and method for operationalizing it allows us to delimit meaningful organizational forms to understand resource and value flows in an econosystem. This is important at the level of applied science, for policymakers who wish to manage econosystems under their jurisdiction. One such econosystem of interest are LRFSs. Specifically, policymakers with purview over the foodsheds in which these systems operate (socially delimited regions in geographic space) often identify policy interventions that can increase the scale of these systems. Applying the OSC to determine species facilitates an ecological approach to such management by allowing us to identify the effects of different nodes within supply-value networks that accomplish scaling up and to target interventions to support those nodes that meet our objectives. 


\subsection{Tables and Figures}

Figure 3.1 Conceptual Framework of the Social Ontology of Organizational Forms

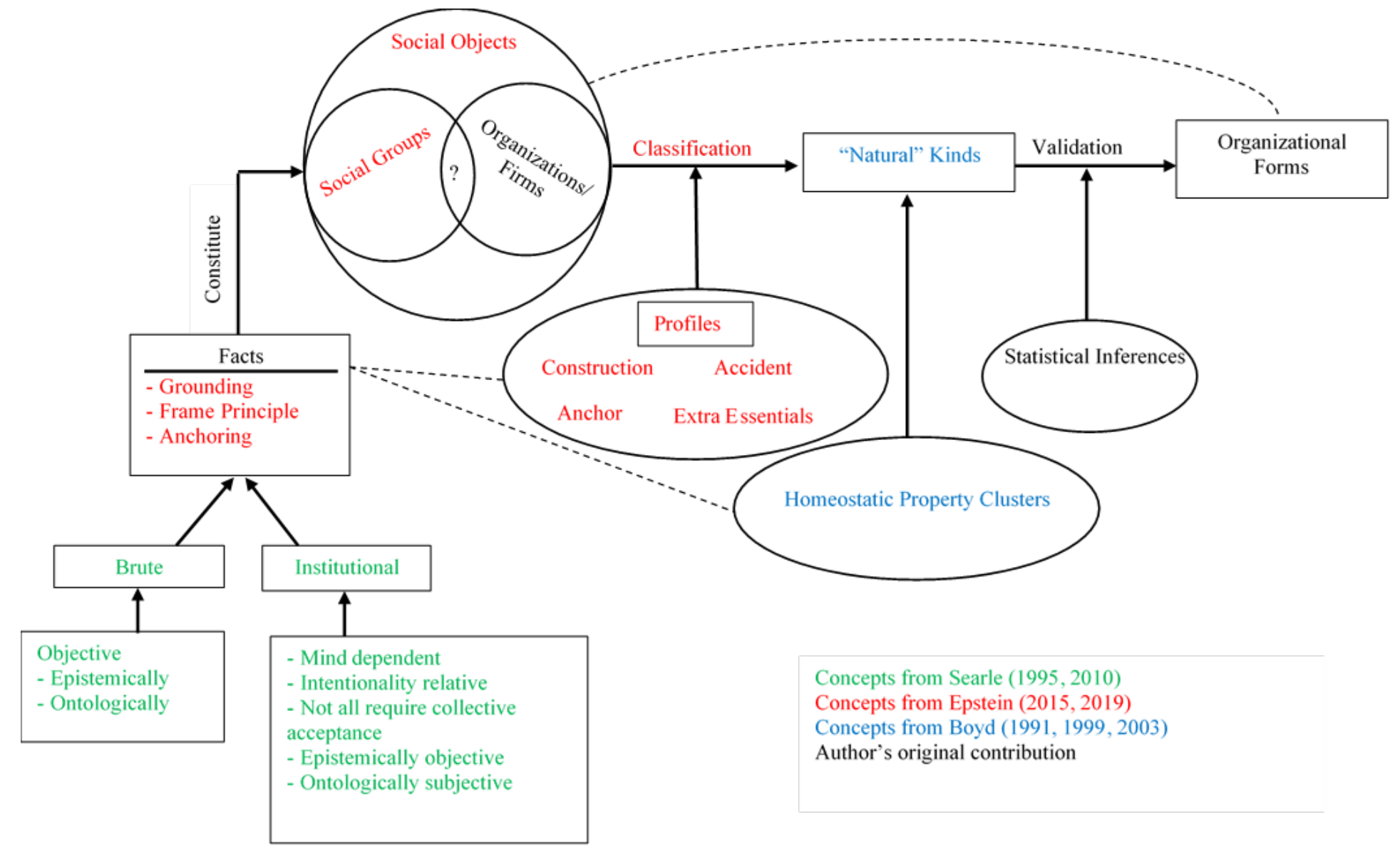

Source: Author. 
Figure 3.2 The Ecological Niche in Reduced-Dimension Resource Space

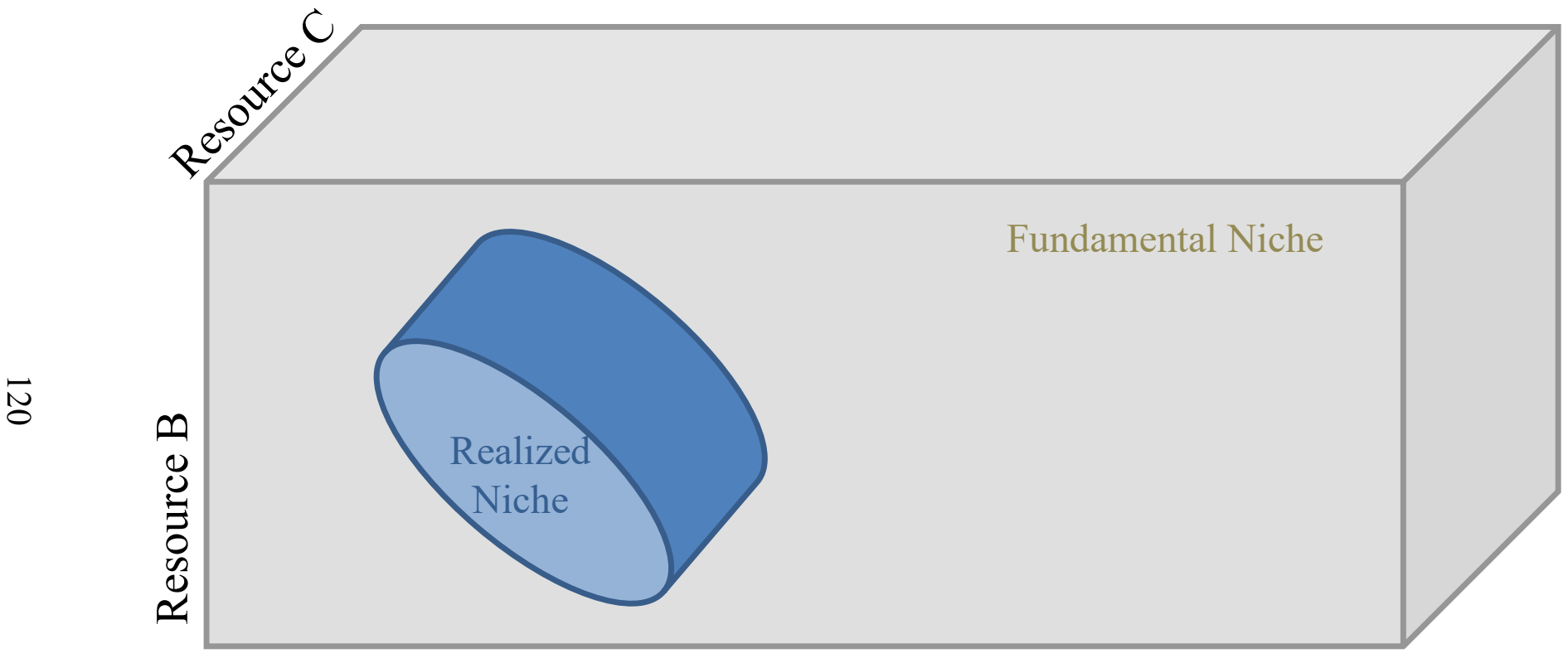

\section{Resource A}

Figure 3.2 depicts the environment reduced to three of $\mathrm{n}$ possible resource dimensions. The fundamental niche represents the boundaries of the resource space in which a species may persist. The realized niche represents the space actually occupied by the species as a result of competitive pressures. 
Figure 3.3 Overlapping Fundamental Niches in the Ecological Hyperspace

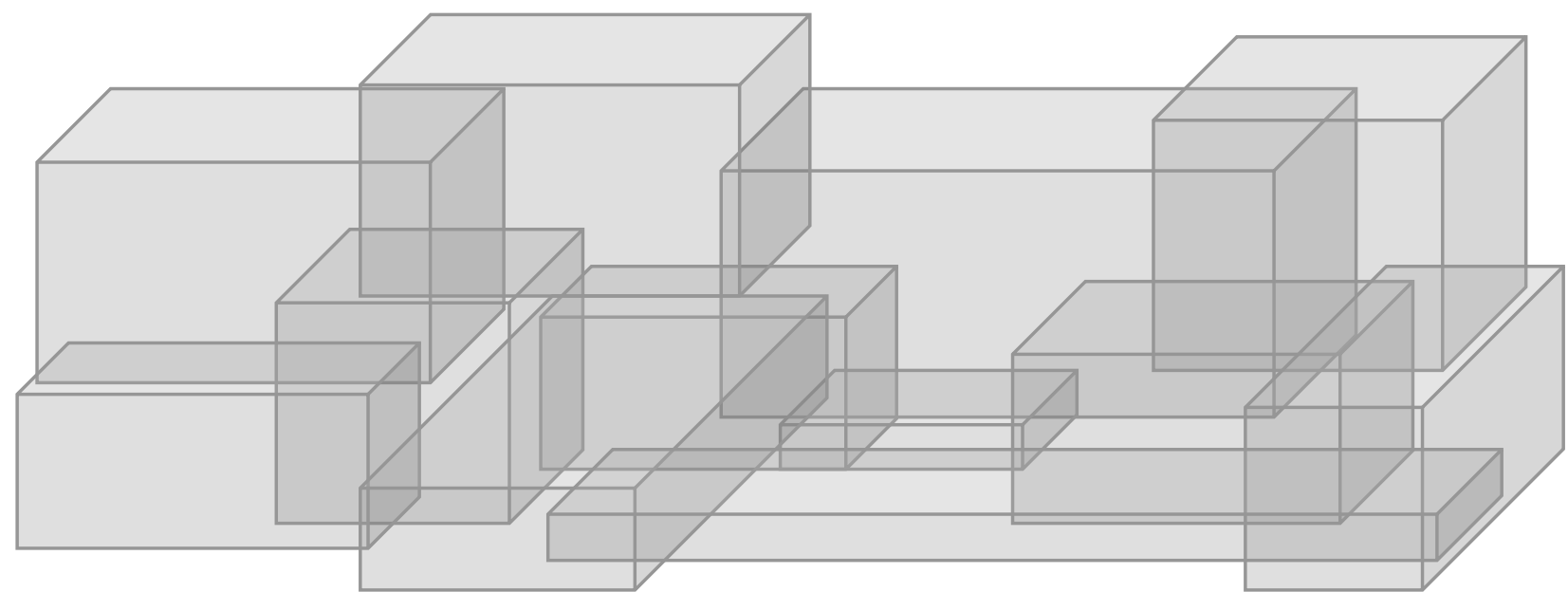

Figure 3.3 depicts an ecological hyperspace with overlapping fundamental niches, each reduced to three of $\mathrm{n}$ possible resource dimensions. Any given fundamental niche is the boundaries within resource space in which some given species may persist. Realized niches will result from species competing for resources within the fundamental niches. Competition is shaped by overlap of the fundamental niches. Figure 3.2 depicts the environment reduced to three of $\mathrm{n}$ possible resource dimensions. The fundamental niche represents the boundaries of the resource space in which a species may persist. The realized niche represents the space actually occupied by the species as a result of competitive pressures. Based on discussion in Hutchinson (1957).

Source: Author 
Table 3.1 Four Categories of Niche Construction

\begin{tabular}{|c|c|c|}
\hline Inceptive & $\begin{array}{c}\text { Perturbation } \\
\text { Organisms initiate change by modifying the } \\
\text { environment }\end{array}$ & $\begin{array}{c}\text { Relocation } \\
\text { Organisms expose themselves to new } \\
\text { environments by relocating in space }\end{array}$ \\
\hline Counteractive & $\begin{array}{c}\text { Organisms counteract a change by } \\
\text { modifying the environment }\end{array}$ & $\begin{array}{c}\text { Organisms respond to a change in the } \\
\text { environment by relocating in space }\end{array}$ \\
\hline
\end{tabular}

Source: Adapted from Odling-Smee and others' (2003, p. 47) summary. 
Figure 3.4 History of the Niche Concept in Biology and Sociology

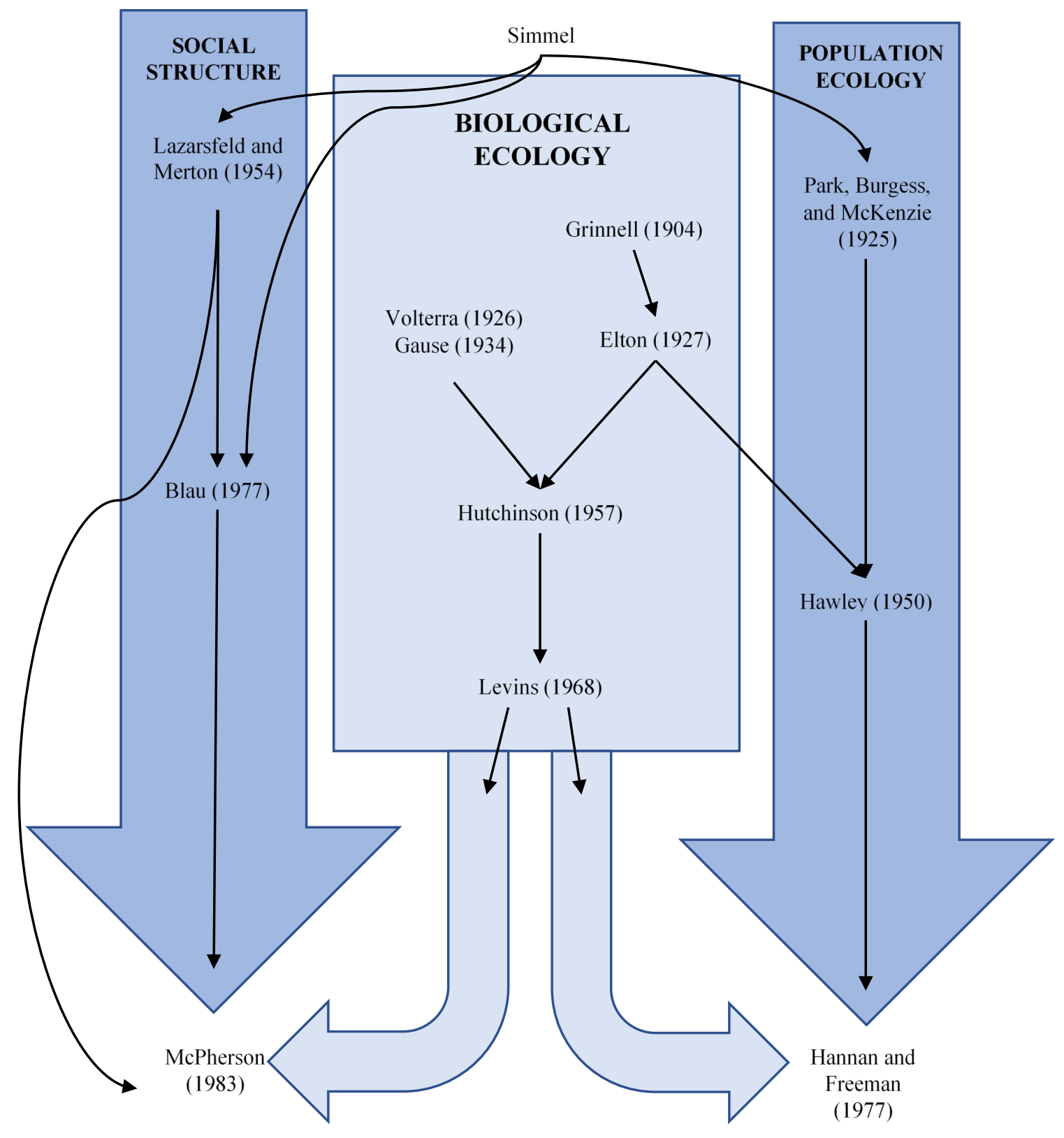

Based on Figure 1 of Popielarz and Neal (2007; pp. C-1). Original extended caption: "A schematic representation of the development of the niche from its origins in biology to use as a theoretical tool in two principal sociological traditions. Dates refer to papers or books that were either first or most seminal at each point in this development. Arrows indicate actual working relationships, citations, and/or intellectual affinities." 


\section{CHAPTER 4 \\ APPLYING THE ORGANIZATIONAL SPECIES CONCEPT: CLASSIFYING FOOD HUB ORGANIZATIONS}

The organizational species concept (OSC) I present in Chapter 3 pivots on developments in philosophy of science as well as on allegories made in the organizational sciences to ecological and biological understandings of kinds. I first examine intrapopulation variety of structural features of firms and search for clusters of homeostatic properties. I then look to behavioral patterns, using the strategic orientations of firms, to validate the resulting kinds as meaningful groupings of cases. This approach evokes a conceptual framework that has been proposed often in the literature, but infrequently tested through empirical means: the organizational niche. This procedure yields the organizational forms, and using the same set of cases - now grouped into forms - we can examine the up- and downstream connections, performance, and other key outcomes to determine how the various forms contribute to policy goals, such as scaling up local and regional food systems (LRFSs).

In this chapter, I apply the OSC to a sample of organizations that self-identify as food hubs to determine the potential boundaries of organizational form in the population. Does a single organizational form encompass all food hub organizations (FHOs), or are there many? Food hubs have been identified as a keystone in endeavors to scale up LRFSs, increasing the volume, value, inclusivity, and presence of place-identified food products transmitted through channels that preserve the ethos of the overarching social movement (Clancy and Ruhf 2010; King and others 2010; Diamond and Barham 2012; Johnson, Aussenberg, and Cowan 2013; Diamond and others 2014; Clark and Inwood 2016). Apart from this pragmatic consideration, FHOs are also a nascent concept and newly established, providing an intriguing space for foundational research on the 
processes of evolution, population ecology, and economic coordination. The sample of cases for investigation is taken from the 2015 National Food Hub Survey. This underlying data set for applying the OSC to FHOs follows a theoretical primer describing food hubs in concept. Summary results of the iterative cluster analysis procedures are presented, with discussion of the taxa I identify and their strategic profiles. Implications of this work follow in a section discussing the results of my investigative work and in one presenting concluding remarks.

\subsection{A Primer on Food Hub Organizations}

It takes only an evolutionary instant for a new organizational form to arise. One such instant has come from the burgeoning of LRFSs with the establishment of food hubs. In less than a decade we have seen a dramatic increase in interest in this new arrangement. Although evidence suggests that self-identified food hub organizations may have been operating for up to 50 years, the majority of those existing today have been in operation for less than a decade. For example, in the 2015 US National Food Hub Survey, one-third of responding food hubs were founded between 2013 and 2015, and 75 percent of responding FHOs were 10 years old or less.

Federal, state, and local policymakers, consumers, primary agricultural producers, and various agents along the food supply chain have grown increasingly interested in these food hub organizations (FHOs). Governmental bodies have directed considerable resources to establishing new FHOs (Fridman and Lenters 2013; Cleveland and others 2014). One early policy development which contributed to the momentum on investment in FHO establishment was the Know Your Farmer, Know Your Food (KYF2 or KYF²) initiative of the USDA (Barham and others 2012). Despite this increasing momentum little academic work studying them is available (Matson and Thayer 2013), likely 
because FHOs are still a relatively new organizational population. The exact origins and history of this innovation are elusive, as are clearly articulated and concrete definitions. However, some basic features of the organizational form have been identified among the otherwise disparate conceptions (Barham 2011; Horst and others 2011; Barham and others 2012; Blay-Palmer and others 2013; Cleveland and others 2014; Fischer, Pirog, and Hamm 2015). FHOs are distinct from the more ubiquitous farmers markets and community-supported agriculture subscription plans (Martinez and others 2010; Low and Vogel 2011; and Low and others 2015). Collective entrepreneurship and cooperative economic engagement are central to food hubs. Thus, when considering FHOs from the standpoint of organizational economics, drawing from the literature on collective economic endeavors, agricultural cooperatives, and other such work is beneficial. Given the various means by which FHOs are founded, we must also fill the gaps in the food hubs literature with what has been said in parallel tracts about statal or quasi-statal agents, both as stakeholders to economic organizations and acting themselves as economic organizations.

Moreover, this new organizational form is not yet fully codified by the institutionalization processes that dominate the research traditions of population ecology (see Hannan and Freeman 1977, 1984; Swaminathan and Delacroix 1991; Hannan and Carroll 1992; Carroll and Swaminathan 2000) and social dynamics (see McPherson and Ranger-Moore 1991; Baum and Singh 1996; Ruef 1997; Stern 1999; Péli and Bruggeman 2005). Some assert that the heterogeneity of structure and strategy in the population of food hubs remains high (Stroink \& Nelson 2013) and isomorphic pressures have not yet sorted the population to the degree that prior work requires for time-series analysis. To 
study food hubs at this juncture requires an approach that celebrates variety in the organizational form but which nonetheless searches to verify whether patterns of structures and behaviors exist among the population. In other words, we must determine whether some identifiable categories exist in the population by using cluster analysis of structural attributes matched with, and validated by, behavioral outcomes.

\subsubsection{The Basic Concept of the Food Hub Organization}

Primarily an North American phenomenon, members of this emergent organizational population serve a role in linking a diffuse, small-scale sector of place- or source-identified food production attached to a highly fragmented food consumption sector. Direct to consumers (DTC) food hub sales are common, but these may also be direct to retailers and restaurants (DTR) sales; sales that are direct to institutions (DTI); such as hospitals, primary and secondary schools, and university kitchens; and direct to other intermediaries including processors and other distributors (DTM). By all prevailing definitions, a food hub is established with an explicit, social mission. Some are directed upstream $^{50}$ (to the farms, ranches, and other primary producers supplying the food hub) and others are focused downstream ${ }^{51}$ (to the end users). These social missions all seek to enact the ethos of the local foods movement and in establishing LRFSs - networks of food production, transformation, distribution, and consumption that provide a socially embedded alternative to the conventional system (Beus and Dunlap 1990; Barham 1997; Hinrichs 2000; Sage 2003; Feagan 2007; Higgins, Dibden, and Cocklin 2008; Feagan and

\footnotetext{
${ }^{50}$ For example, providing market access to minority and other socially disadvantaged farmers or to smallholders whose resource constraints would otherwise limit connections to buyers.

${ }^{51}$ For example, delivering fresh produce to urban food deserts or to school lunch programs, raising nutritional attainment by poor, urban households.
} 
Morris 2009; Starr 2010; Eriksen 2013; Thilmany McFadden 2015; and Barbera and

Dagnes 2016).

Preliminary research, both from reviews of literature and qualitative interviews

completed to date, has indicated the following as the predominant facets of FHOs:

- Act as a clearinghouse for information, providing a conduit for value creation and capture

- $\quad$ Provide a coordinating or governing body for collective action for value capture through an assortment of goods with attributes that give value premiums over conventional channels; economies of scale, scope, and size; collective branding (including creation and enforcement of standards); and collective bargaining

- Minimizing or mitigating certain types of transactions costs

- $\quad$ Balancing economic and social objectives (that is, a hybrid objective function)

Thus, FHOs lie at the center of supply/value chains, forming connections between producers (usually small and medium firms) and consumers (individuals and private and public organizations), while providing an array of social benefits related to building the fabric of alternative food systems (AFSs). It is no surprise, then, that definitions that frame the concept of "food hub" posit that these intermediaries are characterized as (1) playing a coordination role, (2) being part of LRFSs by having a "commitment to place," and (3) typically having a multistakeholder approach.

Marketing channels that include FHOs fall within the class of short food supply chains, discussed in the European literature ${ }^{52}$ in that (1) they have limited steps and limited geographic scope, (2) the goods involved are primarily or exclusively agricultural products intended for human consumption, and (3) they move these goods from producer to final consumer while (4) preserving the identity of products (that is, conveying

\footnotetext{
${ }^{52}$ Here, I drawn upon the literature in Europe, where works by Renting, Wiskerke, Brunori, van der Ploeg, Marsden, and others have expanded this concept. In general, the shortness of a chain is evaluated along three dimensions of proximity: geographical, social, and economic.
} 
information about them along the chain) in ways that provide a premium. FHOs are highly heterogenous including among legal structure, modes of governance, and business strategies among many other firm attributes. To date, attempts to classify within this heterogeneity have relied on a priori, monothetic heuristics that lead to typologies of the sort that have been criticized in the philosophy of science literature as conceptual models of classification, rather than the discovery of kinds. This is to say that, so far, researchers studying FHOs have only classified the diversity of the population by forming categories that are driven by some preexisting theory of differences and that require every case within a group to have every single one of the attributes to the same exact degree. This is exactly the kind of approach that is seen as oversimplified and which fails to account for recent developments by philosophers studying the metaphysics of social objects.

Barham (2011) provides an early working ${ }^{53}$ definition of FHOs that regards a food hub as a "centrally located facility with a business management structure facilitating the aggregation, storage, processing, distribution, and/or marketing or locally/regionally produced food products" (ibid., p. 6). This would soon be put in stark juxtaposition by the now-predominant approach to food hubs as social objects—organizations or networksthat likely have, but are not required to hold, a physical facility, or even a business management structure. In a 2012 document, the USDA adopted by proxy a definition first crafted by the National Food Hub Collaboration, a working group of different stakeholders (Fischer, Pirog, and Hamm 2015). That definition lays the boundaries of what constitutes a food hub: "a business or organization that actively manage the

\footnotetext{
${ }^{53}$ The publications stresses that at publication the US Department of Agriculture was "working with its partners to refine this definition. This is NOT an official [US Department of Agriculture] definition" (Barham 2011, p. 6).
} 
aggregation, distribution, and marketing of source-identified food products, primarily from local and regional producers, to strengthen their ability to satisfy wholesale, retail, and institutional demand" (Barham and others 2012, p. 4). It should be noted, however, that Barham and others (2012) also attenuate this more concise definition with a longer, bulkier explication (see Figure 4.1).

The report of the 2015 National Food Hub Survey adopted core elements of this definition, shortening it to "businesses that actively manage the aggregation and distribution of source-identified food products" (Hardy and others 2016, p. 4). In an early review of the literature, published the same month as the work by Barham and others (2012), Lerman, Feenstra, and Visher (2012) take a different approach: “[b]usinesses that provide aggregation and distribution services to [values-based supply chains] or that otherwise provide new wholesale channels or non-direct marketing strategies to move values-based products are often called food hubs or, more generally, [values-based supply chain] enterprises" (p. 1). This approach is more restrictive in that it excludes direct sales and more expansive in that "values-based supply chains" may be interpreted as including but not requiring an element of locality or source. However, because later in the same work, the authors contradict these definitional elements, I discount their role here.

Blay-Palmer and others (2013) define food hubs as "networks and intersections of grassroots, community-based organizations and individuals that work together to build an increasingly socially just, economically robust, and ecologically sound food systems that connect farmers with consumers as directly as possible" (p. 524). Stroink and Nelson (2013) streamline Blay-Palmer and others (2013), claiming that in the latter "food hubs 
were defined as community-based initiatives that link producers and consumers as directly as possible (p. 620). Stroink and Nelson (2013) perceive Blay-Palmer (2013) as "similar to, but broader than," the definition proposed by Barham and others (2012) and largely base their own approach to food hubs on this conception. Informed by a set of case studies completed with food hubs in Northern Ontario, Canada, which they contend are "more in the initial stages of development... relative to those in the USA," Stroink and Nelson (2013) further explain how they draw the boundaries:

[F]ood hubs represent activities of production, marketing and distribution, or some combination that have a place-based focus on strong linkages between food and health, social justice and building social enterprise operations within local communities. These food hubs contrast with the dominant industrial food system that focuses primarily on efficiency in production and profit to the general neglect of social, health and community outcomes. While there is this commonality in focus among the Northern food hubs, their distinct approaches reveal the complexity of local food hubs and their patterns of emergence in a local food system. (p. 621)

All of these, to varying extents, echo early foundations laid down by Horst and others (2011), who delineate as follows:

A food hub serves as a coordinating intermediary between regional producers and suppliers and customers, including institutions, foodservice firms, retail outlets, and end consumers. Food hubs embrace a spectrum of functions, purposes, organizational structures, and types, each of which can be tailored to achieve specific community-established objectives. Services provided by a food hub may include and are not limited to aggregation, warehousing, shared processing, coordinated distribution, wholesale and retail sales, and food waste management. Food hubs contribute to strengthening local and regional food systems as well as to broader community goals of sustainability and health. (p. 224)

Working with this background, Fischer, Pirog, and Hamm (2015) attenuate both the bulky, behemoth definitions of Horst and others (2011), Blay-Palmer and others (2013), and Stroink and Nelson (2013) and the overly streamlined approaches of Barham and others (2012) and Hardy and others (2016) with a more succinct but highly nuanced 
vision. "Food Hubs," they write, "are, or intend to be, financially viable businesses that demonstrate a significant commitment to place through aggregation and marketing of regional food" (Fischer, Pirog, and Hamm 2015, p. 97). In forming this definition, Fischer, Pirog, and Hamm (2015) rely, in part, on data collected in the 2013 National Food Hub Survey on mission statements of food hub organizations in the United States. The report on the 2015 National Food Hub Survey reaffirms these elements through updated data on mission statements, concluding that "commitment to community through the distribution of locally and regionally produced foods is the critical value that separates food hubs from other businesses occupying the same food supply chain space" (Hardy and others 2016, p. 46).

In their renewed definition, Fischer, Pirog, and Hamm (2015), who were also coauthors of the 2015 National Food Hub Survey report, explicitly reject the use of "distribution" in a definition of food hubs, "to note that the types of customers food hubs sell to (wholesale versus retail) are a less defining feature of a food hub than is the fact that it aggregates food from multiple producers for those customers" (p. 97). Also of particular note, Fischer, Pirog, and Hamm (2015) articulate an explicit understanding of "place," saying that "[t]his term uses Hudson's idea of place as a construct composed of both the production and consumption of material objects as well as social relations within a region" (p. 97, emphasis in original). They stress that place is not simply a physical geographical space but that it also entails the active "creat[ion] of attachments to a community as the location of the socialization process and as a shaper of identity" (ibid., p. 97, citing Johnstone and Lionais 2004). Thus, this definition of a food hub imbues the organization with a relational element that transcends the economic motivations of its 
constituent agents. Adopting the framework of Epstein (2015), the social object that is a food hub is defined by economic and sociological facts - what are collectively social facts.

Although perhaps inherent in Fischer, Pirog, and Hamm's (2015) definition, one explicit element is missing that was present in much of the heftier approaches. Stated succinctly stated by Hardy and others (2016), “[food hubs are] vectors of economic growth and social and environmental change" (p. 4). Missing from the nuanced approach of Fischer, Pirog, and Hamm (2015) is conceptual language that undoubtedly roots food hubs in the realm of sustainability, unequivocally evoking the three pillars or spheres of the most commonly referenced model of sustainability (Figure 4.2 represents this conceptual model).

Many of these features are supported by the (scant) data on food hubs in the United States. The most comprehensive, broadview data come from the two existing iterations of the biennial National Food Hub Survey conducted in 2013 and 2015. The feature of being rooted in a limited geographic scope is stressed by the data from 2015 , for example, in which constituent stakeholders of food hubs were overwhelming from within a 400-mile radius ${ }^{54}$ of the organization's central location; 90 percent of supplying farms and ranches and 75 percent of customers were within this radius in that study. Organizational objectives being more than purely economic and an emphasis on collectivity are also supported. The executive summary of the 2015 report notes that "[m]ore than $87 \%$ of food hubs work to increase access to healthy or fresh food," "[m]ore

\footnotetext{
54 This is one of two criterion in the federal definition of "local" for the purposes of local foods as per the Food, Conservation, and Energy Act of 2008 (Martinez 2010).
} 
than $95 \% \ldots$ work to improve human health in their communities or region," and they "turn to communities of practice and networks for information" (p. 5).

That same set of survey reports provides additional first-level understanding of food hubs, especially stressing the heterogeneity of organizations that identify as such. As is widely asserted in the literature, food hubs are a nascent form, with the 2015 National Food Hub Survey respondent organizations having a mean age of 8 years and a median age of 4 years. Of the respondents, 63 percent had been operating for 5 years or less. These organizations operate under as many as 11 different legal structures (see Figure 4.3). The majority of food hubs also have heterogeneous consumers, adopting a hybrid business model in that they sell simultaneously to wholesale and retail buyers. Food hubs also rely on rather heterogeneous input sources. Primary producers (farmers and ranchers) are the most frequent type of supplier; data from Hardy and others' (2016) report (reproduced in Figure 4.4) shows a small number of hubs that do not source directly from this group. Moreover, many hubs source product from multiple sources, with a wide spread in the number of suppliers per food hub, ranging from 3 to 1,500, with a mean of 83 and a median of $36 .^{55}$ This story of heterogeneity continues into the product categories carried by hubs, with retail-oriented hubs carrying products in an average of eight categories, hybrids an average of five, and wholesale-oriented hubs an average of four. Fresh produce is, however, the predominant product category, with 92 percent of hubs dealing in these products in 2015, accounting for 63 percent of gross sales to the average hub.

\footnotetext{
55 The 2013 and 2015 National Food Hub Survey reports do not provide supplier breakdowns in the legal structure and business models; these data may explain how different organizational types are constituted.
} 
One final section of information from the 2015 National Food Hub Survey is worth noting in this review. Data on financial performance of food hubs indicates a potential striking difference in performance of these organizations in their legal structure and business model. In the 2013 and 2015 samples, hubs structured as cooperatives largely outperformed for-profit and not-for-profit structures on average, with mean revenues of $\$ 3.01$ million and $\$ 1.29$ million more than that for for-profits (the secondranked type) in 2013 and 2015, respectively. The maximum in the range of revenues for cooperatives were also an average of $\$ 23.15$ million higher than that of for-profits across the two survey years. ${ }^{56}$ In the 2015 data, cooperative-structured hubs also had the lowest mean, median, and maximum operating expense ratio of the three types. ${ }^{57}$ Likewise, the 2015 National Food Hub Survey data indicates that hubs with a wholesale-oriented business model may outperform hybrid and retail-oriented hubs with mean ( $\$ 6.62$ million difference), median ( $\$ 807,000$ difference), and minimum ( $\$ 43,000$ difference) and maximum ( $\$ 79.5$ million difference) revenues being greater than the second-ranked category ${ }^{58}$ for each measure. ${ }^{59}$

The preceding discussion makes apparent that, in formulating the membrane that constitutes a food hub as an economic organization, emphasis is placed on functions and orientations, with significant allowance for heterogeneity in legal formulation, governance structure, and operational cadre. These boundaries attribute economic and

\footnotetext{
${ }^{56}$ Median revenues for cooperatives in both survey years were significantly lower than for-profits by $\$ 224,000$ and $\$ 754,000$ in 2013 and 2015, respectively. The minimum in the range of revenues is also lower for cooperatives compared with for-profits but by a smaller margin of $\$ 6,000$ and $\$ 8,000$, respectively.

${ }^{57}$ Comparisons with 2013 were not presented in the 2015 report.

${ }^{58}$ The second-ranked category for mean revenue is retail-oriented hubs; for median, minimum, and maximum, it is hybrid hubs.

${ }^{59}$ These data are available only for the 2015 survey; the question on business model was not asked in the 2013 survey.
} 
social roles to food hubs, roles that may come into conflict or require balancing internal to the organization. We must recognize that profit, let alone its maximization, "is not always the primary underlying motivation for establishing a food hub" (Matson, Shaw, and Thayer 2016, p. 7). Thus, we must also incorporate understanding from the broader literature on social and solidarity economy. It is also clear that heterogeneity along various lines meaningfully affects the economic performance — and thus, by extension, the longevity — of organizations that identify as food hubs. To date, the predominant definitions have incessantly been driven for an all-encompassing, amorphous concept that ignores meaningful differences.

\subsubsection{Economic Organizations Rooted in a Social Movement}

The literature on definitions makes clear that establishing food hubs is closely linked to a notion of geographic and sociological place as a reaction by a subset in communities to consolidating the food system and expanding food supply chains. To this point, Stroink and Nelson (2013) clearly center the founding of food hubs in Northern Ontario as part of a social movement—one that aims to build a system that emphasizes local, regional, and traditional foods — as a counter to the "industrial" food system. In adopting a complex adaptive system approach, they see a series of nested relationships and mutual connections; food hubs are systems per se that nested within a wider $\mathrm{AFS}^{60}$ whose creation is coordinated and catalyzed through a social movement.

Other authors also use language, frameworks, and imagery that inculcate food hubs as an organizational form with origins and establishing motivations in social movements. Matson, Shaw, and Thayer (2016) locate the "emergence and evolution" of

\footnotetext{
${ }^{60}$ The alternative food system is nested in several macro-level systems, including the entire food system, and is parallel to - and influenced by the existence of - the industrial food system.
} 
these organizations in "an educational and social mission to unite consumers and producers in the marketplace" (p. 6). In other words, they arise from a "mission" to shorten food supply chains and coordinate the two extremities. Cleveland and others (2014) posit food hubs as "often a key component" ${ }^{61}$ of AFSs developed "as a way to reduce [the externalities of the 'mainstream' food system] and increase food system environmental and social sustainability," for which "[1]ocalization has become a favoured strategy" (p. 26). They continue by invoking the well-known framework of Polyani (2001) to define AFSs as a social countermovement. The treatment of economic organization founding as, in part, an element of a social movement is not foreign to the economics and management literature either. Boone and Özcan (2014) examine the formation of collective economic organizations - cooperatives in the United States biofuels industry - as a part of a social movement that counters corporatist control of the industry. According to their description, the movement exhibits strikingly similar features to those that are described in the aforementioned treatments of food hubs. Boone and Özcan (2014) note that "social movements [mobilize] economic and sociopolitical resources" required as catalysts for organizational founding and continuance (p. 993).

Given sufficient justification to root the formation of food hubs in a social movement, one must consider the definition and features of that movement. Many names exist for the generalized patterns of behaviors and attitudes in the literature on food hubs; "locavore," local foods, alternative food systems, "food glocalization," "civic agriculture," and many more are scattered throughout the literature that touches on these

\footnotetext{
${ }^{61}$ Food hubs are key, they contend, because they present a strategy to address a major obstacle to localization: "the lack of economic, organizational, and physical structures of the appropriate scale for local aggregation and distribution of food" (p. 26).
} 
attributes. Some even express uncertainty on whether any of these coalesced attributes constitute a movement. As Starr (2010) ponders, "Is local food a set of policies, a consumer fad, a new market, or a social movement?" For it to be the latter, Starr asserts, it must meet two thresholds: (1) formulating a "we" (a common identity) that is sustained, and (2) creating a "new idea." She concludes that the thresholds are met and that there is a local food social movement. This is only one concept of the elements defining a social movement; Weber, Heinze, and DeSoucey (2008) provide a succinct discussion of alternative ways of conceiving what constitutes a social movement. ${ }^{62}$ Starr's (2010) analysis is one of the most in-depth that explicitly treats the local food movement, but other concepts are likely to lead to similar conclusions.

The boundaries of that movement, however, still remain to be delineated. In the context of the food hubs and related literature, I propose a minor expansion to "local and regional food systems movement." This adopts some of the expanded nomenclature common to both the food hubs literature and the general preponderance of the USDA's approaches to policy (see, for examples, Martinez and others 2010; Low and Vogel 2011; Low and others 2015; Berti and Mulligan 2016). Granted, even adopting this expanded naming convention for the social movement does not fully delimit it; "local" and "food" have competing and sometimes contradictory definitions (Feagan 2007; Darby and others 2008; Martinez and others 2010). Also ill-defined are the hallmarks of this movement and how those features manifest themselves in the economic organizations we call food hubs.

\footnotetext{
${ }^{62}$ Weber, Heinze, and DeSoucey (2008) significantly depart from Starr's (2010) implication that a social movement and a new market are ontologically distinct. The former's entire analysis shows that a social movement is a catalyzing — and possibly necessary — element for creating new markets or market segments and niches.
} 
On the first point, from a US policy stance, one definition of "local" has been codified in law through the Food, Conservation, and Energy Act of 2008. This definition sets geographical and legal boundaries: being within a 400-mile radius of a product's origin and being within the state in which a product is produced (Martinez 2010). What is understood as a legal definition and what is understood as legitimate in a movement of consumers and producers may differ (Darby and others 2007).

On the second point, experts in the field disagree on what constitutes the social movement of interest to us. Starr (2010) conflates as one the local and the "organic" but does not provide sufficient empirical support for this element. This conflation is countermanded by other studies of the local and regional food movement. For example, Winter (2003) and Hinrichs (2003) posit concepts of the local and regional food movement that are distinct and independent from those with specific production practice attributes. Qualities such as "grass-fed" and "organic" can still be attributes of the movement of interest, but they are not defining attributes; these attributes have their own movements with networks and concepts that overlap with those of the local and regional food systems movement. ${ }^{63}$ The aforementioned definitions help to identify many of the ornamental features of the food system; limitations to region, orientation to community, improvements in economic equity and justice, and relatively short supply chains are all good candidates. However, more extend beyond the definitional elements. Winter (2003) and Hinrichs (2003) provide evidence for adding defensive localism. Unfortunately,

${ }^{63}$ The complex adaptive system framework discussed by Stroink and Nelson (2013) helps to conceptualize this. Under this framework, the local and regional foods, organic, grass-fed, and other movements are the social coalitions that form to establish distinct but parallel or layered systems nested in a wider alternative food system. 
comprehensive analysis and testing of these elements requires empirical analysis and theoretical work that as of yet is not available in the literature. However, doing so is paramount to understanding not only the social movement but also its effects on the resulting systems and pertinent social objects.

This importance can be seen in the work of Weber, Heinze, and DeSoucey (2008), which examines how the movement for grass-fed (that is, a specific production practice) affects cultural change by creating new markets or market segments. ${ }^{64}$ They posit that social movements can overcome three challenges of creating these new market segments: (1) "creating" entrepreneurial producers (supporting motivation, commitment, and innovation), (2) creating collective producer identities, and (3) establishing regular exchange between producers and consumers. Here, I stress the second item, as these collective identities "give rise to cooperative efforts to institutionalize the market category" (Weber, Heinze, and DeSoucey 2008, p. 547). Social movements create cultural change, and thus often lead to establishing entirely new organizational forms. This is much like the process of identity formation and legitimation described by Carroll and Swaminathan (2000) under the craft brewery movement, which Weber, Heinze, and DeSoucey (2008) reference. Thus, social movements are one method communicating an emerging identity and legitimizing organizations that fit within that identity. What Weber, Heinze, and DeSoucey (2008) do not discuss (and which is beyond the brief of their analysis) is how these identities shape the choices on the structure and nature of the

\footnotetext{
${ }^{64}$ Weber, Heinze, and DeSoucey (2008) adopt a "micro-constructionist" perspective and carefully explain how this perspective differs from and in part counters the micro-economic perspective on markets. "The micro-constructionist perspective... suggests that markets function when the participants in exchange...establish a stable social organization with roles and niches" (p. 531; citations omitted).
} 
resultant organizations. Despite limited research on these matters, it is worth noting the question, which presents a complex problem, not the least of which is a chicken-or-theegg matter of sequence. ${ }^{65}$ In the next section, I examine the more prolific literature on economic rationales for organizational structure. Given the lack of research on the role of organizational identity (which is clearly imbued, at least in part if not in whole, by the social movement's ideological features) in these decisions, we cannot easily dismiss the organizational identity as having no role in these processes. It most likely plays a moderating or mediating function.

\subsubsection{Hybrid Organizations: Economic Theories of Existence}

In the literature on food hubs, the word "hybrid" is often used to describe the organizations. However, the use of this term in relation to food hubs varies dramatically. Cleveland and others (2014), for example, conflate two notions of hybridization, which I separate as a hybrid identity ("idealistic" vs. "instrumental" in their terms, "normative" versus "utilitarian" in others) and hybrid framing (operating in an "alternative" versus “mainstream" food system). In the 2015 National Food Hub Survey, Hardy and others (2016) use the term on what I call a hybrid business model basis (selling to wholesale versus retail markets). In a unique approach to the notion of hybridity, Klein and Michas (2014) consider what I term hybrid chains. Considering a case of the common healthcare institutional buyer, they come to food hubs from the same values-based supply chain position as do Lerman, Feenstra, and Visher (2012). Thus, for Klein and Michas (2014), a hybrid (values-based supply) chain model "incorporate[s] both conventional and

\footnotetext{
${ }^{65}$ In the field of confirmatory factor analysis, this is alternatively presented as a matter of formative versus reflective. That is, does identity shape the choices, or do the choices shape the identity? Likewise, does the movement create the identity characteristics, or do identity characteristics act as indicators of the movement?
} 
alternative resources, infrastructure, and markets." Other elements in the literature approach various similar adaptations.

It is interesting — and shocking - that there is no substantive instance in which food hubs are considered or analyzed from the standpoint of a hybrid economic organization. This standpoint is well developed in the economics and strategic management literature and deserves to be applied to food hubs. This failure points to a larger gap in the literature: no identifiable work considers theoretically—let alone examines empirically — the economic rationale for either the establishing food hub organizations per se, demarcating their boundaries, or choosing their organizational structure. We have no explicit economic explanation for why food hubs exist, why they decide to bring what they do in the membrane of the organization, and how and why they choose certain structures.

In classical economic theory, markets bring efficiency, thus obviating bringing functions in the membrane of an organization, as contracting on-the-spot market would always be the most efficient. Reality does not bear this out. In response, competing theories attempt to explain why a firm - which I will term generically an economic organization - comes to exist at all. Mahoney (2005) presents an overview of the five central approaches to theories of the firm: transactions costs theory, behavioral theory, property rights theory, agency theory, and resource-based view. Because of this comprehensive overview, I do not discuss its treatment here but rather stress two central points. First, at the early stages of when theories of the firm were developed, many of the approaches examined a simplistic dichotomy: there was either a market or a hierarchical firm, the latter of which is characterized by a relatively linear command-and-control 
structure that ultimately reports to a singular authority. However, once again, observation shows that economic organizations exist that mix elements of markets and pure hierarchies. This mixture ${ }^{66}$ constitutes the hybridization in which we are interested. Second, the five central theories of the firm, or at least concatenations of several, have led to different concepts and explanations of these hybrid economic organizations. We do not present an exhaustive review of all existing concepts but instead highlight some key approaches and points that provide an introduction.

Williamson (1991) provides one concept. Rooted deeply in the transactions cost approach, Williamson's (1991) model of hybrid economic organizations invokes economizing on these costs as a central objective. His concept pivots upon notions of contract law, with organizational forms being supported by a distinctive theory thereof. Williamson (1991) adopts the common "nexus of contracts" definition of economic organizations and firms seen. Furthermore, for Williamson (1991), the central economic problem relates to adaptation to disturbances. Specifically, he argues that hybrid economic organizations rest upon neoclassical contract law modified by the Excuse Doctrine. Under this contract regime, "[t]he parties to such contracts maintain autonomy, but the contract is mediated by an elastic contracting mechanism," which "better facilitates continuity [of the relationship] and promotes efficient adaptation [to disturbances]" (p. 271). However, this is limitedly so; as Williamson (1991) notes, neoclassical contracts "experience real strain, because the autonomous ownership status

\footnotetext{
${ }^{66}$ Some experts in the literature argue that hybrid economic organizations are distinct forms; in developing an hybrid economic organization concept from a transaction cost approach, Williamson (1991) notes that "the hybrid form of organization is not a loose amalgam of market and hierarchy, but possesses its own disciplined rationale" (p. 294).
} 
of the parties continuously poses an incentive to defect" and "deal with consequential disturbances only at great cost” (p. 273). Thus, for Williamson (1991), hybrid economic organizations are the best organizational form response when there is "semi-strong" intensity of incentives to adapt, reliance on administrative controls, benefits from autonomous adaptation, and benefits from coordinated adaptation.

A decade later, Madhok (2002) critiques and extends Williamson's (1991) concept by integrating it with the resource-based view. These two theories of the firm are then triangulated with the nature of governance structures (market, hybrid, or hierarchy), with the goal of aligning characteristics of the structure, the transaction, and the resources. It is interesting that Madhok (2002) does not mention hybrids - speaking only in the generic term of the choice of governance structure - partly because he focuses on "why a particular firm will/should organize that activity hierarchically within its boundaries" (p. 541, emphasis added). Thus, a systems view of Madhok's (2002) triangular alignment theory allows for hybrid economic organizations. This systems view is implicitly adopted by Hendrikse and Bijman (2002), who develop a theory of marketing cooperatives in agrofood chains with an approach that considers agents in three tiers of the organic dairy supply chain (farmer, processor, and retailer). They attempt to explain why certain structures of vertical coordination are selected in a value chain, approaching the problem from incomplete contract theory, presuming as the motivating factor incentives that arise from the rents generated by individual agents' investments in human capital (knowledge and skills) and ownership of productive assets. It is notable that there is no restriction on these agents having singular ownership of the assets, only that they hold some quantity of residual rights with an associated payoff. 
Through their framework, Hendrikse and Bijman (2002) show that the marketing cooperative ownership structure (that is, the farmer takes residual and control rights of the processing asset, the factory) is the first-best efficient option when the farmer's investment costs are "relatively large compared to the investments of the [other agents]" (p. 112).

Hendrikse and Bijman (2002) were not the first, nor the last to examine cooperatives as hybrid economic organizations (Ménard 2004; Chaddad 2012), an important fact to note given that data indicates a substantial portion of food hubs in the United States are organized explicitly as such. However, not all food hubs are established with the legal form of a cooperative. Does this mean that they are not hybrid economic organizations? This partly empirical question can be sussed out only through research findings that have not yet been conducted. However, as an initial approach, I argue that given the definitional elements of food hubs, the likelihood of most food hubs being hybrid economic organizations is reasonably large. The small selection of models presented earlier notwithstanding to support this, there is another line in the hybrid economic organization literature that may justify an argument that many food hubs - no matter their legal form—should be evaluated as such. Extending the wider opus of Williamson (1991) and others in the literature, Ménard (2004) develops a typology of hybrid economic organizations using an approach that centers around transactions cost theory but with some elements of the resource-based view. For Ménard's (2004) analysis, the question is what type or form of hybrid economic organization will be chosen and what determines the internal properties of such an arrangement. He posits two characteristics of transactions as the pivots: the imposition of mutual dependence (that is, 
bilateral or multilateral reliance) through investment in specific assets and uncertainty about the transactions to be organized. In his discussion, he identifies four types of hybrid economic organizations: trust-based arrangements, relational networks, leadercoordinated arrangements, and formal private governments.

Others have, at times unwittingly, provided similar types of hybrid economic organizations, most predominantly of the relational networks form. (Powell 1990; Podolny and Page 1998; Demil and Lecocq 2006) Some of these, such as Powell (1990) and Podolny and Page (1998) are posited as sociological criticisms of economic theories of hybrid economic organizations. ${ }^{67}$ However, I argue that pitting the two fields against one another does only a disservice to developing a greater understanding on the issues identified in this article on food hubs. Some empirical evidence supports this assertion, such as the work by Boone and Özcan (2014), who empirically test hypotheses that integrate economic and sociological explanations for establishing cooperative-form hybrid economic organizations. Although potentially reasonable criticisms of their work, it nonetheless indicates that there is likely support for the conclusion that economic theories of hybrid economic organizations are valid, but may be attenuated by sociological factors. Boone and Özcan (2014) claim that the presence of a social movement is key to explaining the persistence of cooperative forms that economic theory would otherwise expect not to exist, an assertion that is not unique in the literature reviewed for this exploration. This kind of approach cannot be dismissed, nor can economic theories of hybrid economic organizations, if we are to deepen our

\footnotetext{
${ }^{67}$ However, Powell (1990) notes that in his previous concepts that he had seen relational network organizational governance structures as explicitly part of the hybrid economic organization tranche that fit within economic justifications.
} 
understanding of food hubs' establishment, boundaries, and governance. On the basis of what the literature contains so far, I hypothesize that food hubs are likely to have aspects - similar to cooperatives and other forms of collective entrepreneurship — that are well described by economic theories but that these are attenuated in some way by aspects of the political economy in their establishing and catalyzing social movement. Because of this political economy aspect, it is also imperative to recognize and deal with matters that are related to the agents that constitute these economic organizations.

\subsubsection{Multiple, Heterogeneous Stakeholders}

What is not yet been clearly identified in robust analysis is the nature and degree to which food hubs have "multiple stakeholders," that is, that they are constituted by relationships among agents that are heterogeneous. This is not to mention understanding on the effect such a presence may have in the realms of founding, organizational governance choice, multilevel decision-making processes, and performance and outcomes of food hubs. It is clear, however, that at least some (unknown) proportion of food hubs have varied constituencies within whatever organizational governance structure they adopt. Moreover, if an economic organization is to meet the definitional elements described earlier, particularly those of community and/or place, a multistakeholder nature may well be an assumption, if not an explicit requirement. As Matson, Shaw, and Thayer (2016) note, "the continuous adaptation [of the food hub business model] has resulted in increased focus on the social-mission aspects of many food hubs and their community interactions, as well as a movement to address multiple stakeholder classes in a community" (pp. 5-6). There is a clear need to address the many questions that arise from the multistakeholder aspect in such organizations. Although I discuss that here, the relevant literature is sparse. 
We must first deal with some foundational issues related to multiple stakeholder groups in economic organizations. A number of theoretical approaches can be identified thus far. Coff (1999) approaches multistakeholder economic organizations by integrating the resource-based view, which was designed to explain when firms will generate rents, with a bargaining power model, which enables understanding of who will appropriate rents. Thus, Coff's (1999) work is concerned with understanding the performance of an economic organization that has competing stakeholder groups, which are, in his view, employees and shareholders. Asher, Mahoney, and Mahoney (2005) also present an approach tied to the resource-based view and the same fundamental questions of the generation of "value" by a firm and its distribution among stakeholders. They differ, however, in that they look to integrate with theory of property rights, which they define as including "any social institutions that define or delimit the range of privileges regarding specific resources granted to individuals" (p. 8). Both the Coff (1999) and Asher, Mahoney, and Mahoney (2005) approaches challenge the hegemonic view that the only residual claimants are shareholders (that is, providers of equity). They also still hold many limitations in relation to the applicability to food hubs: (1) there is a presumption that the existence of the firm and its competitive advantage is ascribed to the resourcebased view's elements, primarily knowledge resources, (2) agents' motivations are presumed to singularly focus on rent extraction and maximization, (3) stakeholder groups are implicitly those with "strong" transactional ties to the economic organization such as employees and providers of equity and credit, and (4) their discussion is conducted within the context of economic organizations with corporate forms. 
The literature on cooperatives offers some alternatives and extended discussion that, in part, begins to address some of these concerns. Cook, Chaddad, and Iliopoulos (2003) provide a comprehensive overview of this literature's development, especially that during the 1990s. They categorize this literature into three tranches: cooperatives as firms, cooperatives as coalitions, and cooperatives as nexuses of contracts. Although many of the theoretical models they discuss across the three categories likely do not show promise for extension to multistakeholder organizations, some do. These include approaches where membership subgroups are maximizing utility objective functions in bargaining frameworks and various applications of game theoretical models that explain bargaining outcomes and organizational governance choice. Lund (2012), however, presents somewhat of a watershed in the cooperatives literature as it can be applied to features seen with food hubs. Her explicit treatment of multistakeholder cooperatives takes a value-chain approach (differentiating from the traditional supply chain approach) and posits that multistakeholder enterprises "whereby instead of thinking of the potential high transaction costs of involving multiple parties, it may rather be more appropriate to think of [them] as more highly evolved mechanism for the collection and coordination of disparate information in the pursuit of common needs"68 (p. 33). For Lund (2012), what little literature exists points to multistakeholder cooperatives as being transformationally oriented, not transactionally. Where Lund's work stops short is in not operationalizing these into an economic framework(s) that can be analyzed.

\footnotetext{
${ }^{68}$ She continues by saying that "[o]ther researchers agree, acknowledging the reduced transaction costs that ultimately emerge through the increased levels of information, trust, and involvement resulting from the mutli-stakeholder approach" (Lund 2012, p. 33, citations omitted).
} 
On the basis of general elements in the literature and anecdotal evidence on food hubs, I propose four central categories of food hub agents: statal actors (local, state, and federal governments and their quasi-public agents); community-based and nongovernmental organizations; producers; and consumers. The latter two can be natural and fictitious legal persons. This list is not exhaustive, and a heuristic has not been proposed, although empirical verification is not likely to be difficult. Not all categories will be present in any given food hub organization. When present, their roles may differ across organizations and can be formal or informal. Food hubs may fall within one or more of these categories per se. The relevant concern is the interests and behaviors of the entities that are constituting elements of the food hub and how these affect observable social facts about them. These categorizations enable are more organized discussion of key conceptual points.

Of these categories, statal actors have received the most attention in the literature. These are important for food hubs, with 3 percent of respondent organizations in the 2015 National Food Hub Survey indicating that they were "publically owned," which I interpret to mean that a public agency, such as a local or state governmental authority, holds claim to a majority of either control or residual rights, or both, of the organization. However, no data could be identified on the additional formal and informal roles assumed by such actors in the other legal structures adopted by food hubs, despite anecdotal evidence that these may be significant in some cases. Researchers have approached public entities in social movements in general — and food systems and the organizations within them in particular-from many angles. Empirical evidence supports the conclusion that having public agents in agricultural sector organizations, in so called 
public-private partnerships, can significantly influence those organizations to facilitate improvements in firm performance (McDermott, Corredoira, and Kruse 2009). These public agents form such partnerships by facilitating the independent creation of or directly establishing organizations with the goal of enacting policy prescriptions related to a wide array of social mandates for government (Renting and Wiskerke 2010). Given this, research on food hubs must be sensitive to addressing the possibility that such organizations become a quasi-statal instrument of policy. ${ }^{69}$ This necessitates the integration of political theory into the economic and sociological approaches described previously. Beyond this, the nature and volume of resources being contributed by public agents to the establishment and continuance of food hubs may alter or attenuate the theoretical expectations on founding propensity, selection of governance structure, decisions on boundaries of the economic organization, and performance. Unfortunately, I found no substantive literature that addresses these issues.

Similarly, almost no identifiable literature examines the role of community-based organizations and nongovernmental organizations as stakeholders in economic organizations, especially for the matters of interest here. Some studies in the literature have examined issues of founding for food hubs with an organizational structure of a nonprofit (Diamond and Barham 2012; LeBlanc and others 2014), and there is similarly a wider literature on multiorganizational partnerships (Lowndes and Skelcher 1998) and organizational identity in nonprofits (Young 2001). However, none of these reach deep enough into the organizations to parse out the effects of constituent stakeholder groups.

\footnotetext{
${ }^{69}$ Fridman and Lenters (2013) make this abundantly clear, perhaps unintentionally, as they discuss an investigation of community kitchens as food hubs in Toronto.
} 
Similarly, few studies explore the interplay of producers and consumers as stakeholders

in a single organizational membrane. However, the abstract to Lowndes and Skelcher's

(1998) work is important in that it effectively summarizes the key points of interest:

Multi-organizational partnerships are now an important means of governing and managing public programmes. They typically involve business, community and not-for-profit agencies alongside government bodies. Partnerships are frequently contrasted with competitive markets and bureaucratic hierarchies. A more complex reality is revealed once partnerships as an organizational form are distinguished from networks as a mode of social co-ordination or governance. Data from studies of UK urban regeneration partnerships are used to develop a four-stage partnership life cycle: pre-partnership collaboration; partnership creation; partnership programme delivery; and partnership termination. A different mode of governance - network, market or hierarchy - predominates at each stage. Separating organizational form from mode of governance enables a richer understanding of multiorganizational activity and provides the basis from which theory and practice can be developed. The key challenge for partnerships lies in managing the interaction of different modes of governance, which at some points will generate competition and at other points collaboration.

I did not find this distinction in the economic, sociological, or strategic management perspective literature. Thus, addressing food hubs - at least those that have multiple stakeholder groups - from a partnership perspective may help to explain how these stakeholders interact, especially at different stages of a lifecycle framework, and the effects of these interactions on the questions of interest.

\subsubsection{Putting It All Together: How the Theory of FHOs Drives the Search for Kinds}

The theoretical and conceptual development of the preceding sections charts the course for operationalizing the OSC to identify kinds, if they exist, among the population of economic agents serving roles as coordinating intermediaries within LRFSs. Given the boundaries set forth in Chapter 2, to be part of LRFSs, these agent must seek to enact a "commitment to place." A number of dimensions of proximity can accomplish this. Thus, the search for species must allow for different modalities for making the transactional 
environment in which food products are exchanged proximal between consumers, producers, and place. In doing so, FHOs are likely seeking to maximize a hybrid objective function, trading off between behaviors that are profit seeking with those that are social welfare seeking; the degree, extent, and magnitude of these trade-offs may be a key differentiating feature of the strategy between kinds. Because FHOs are often multistakeholder organizations, their method of organizational governance and decision making (that is, control) and ownership structures is important; differences in these are expected to alter the degree and nature of the FHO's trade-offs between profit and sociality.

Thus, the morphological structures of FHOs should be expected to match the strategic positions that achieve the maximization rule for this objective function; these structures accomplish certain payoffs but also have differing costs associated with them in terms of capital investments, managerial needs, labor, and so forth. So too may different strategic niches, especially in enacting the proximal attributes in the transactional environment, have different payoff structures - within a given strategy space different dimensions of proximity may be seen as more or less legitimate enactments of a commitment to place and have different premiums associated with them in the market. These observations must guide us in selecting both morphological features and strategic orientations, the latter a proxy for the organizational niche. For the former, we should prioritize morphological features with a relative degree of "stickiness," as our search is for property clusters with a homeostatic nature. Structures that are relatively time-invariant are preferred over those which can easily be jettisoned, replaced, or added. Morphological features must account for as many of the most prominent structures within 
an organization that account for the underlying costs and payoffs of the hybrid objective function, while those used in validation should capture this tradeoff in strategic space. Under the OSC, even with morphological differences, if these do not associate with different niches, here operationalized as strategic positioning, then the differences in features are trivial and part of the normal variation within a cohesive population.

\subsection{Data: The 2015 National Food Hub Survey}

To formulate taxonomic structures for a population of firms, a substantial amount of information on individual cases is required. Ideally, this set would include information on resource endowments, infrastructures, operations, behaviors, revenue and expenditure structures, and strategies. The information set should include a sample of cases (instances) that can reasonably represent the population. This is costly to collect de novo, especially for organizational sets where markets are thick and populations are estimated to be large or where record keeping and response rates are low. Finding a secondary data source in such contexts that approximates the most ideal qualities is much more feasible.

Given the developmental stage of FHOs and the carrying capacity of the LRFS environments in which they "live," population size is small. A motivation in a distinct social movement leads to a relatively developed network among firms and creates structures of authority aimed at supporting and promoting the development of these businesses. Moreover, keen interest from industry and policymakers has led to recent investment in the collection of data on a biennial basis by these support networks under the auspices of a National Food Hub Survey program.

This work uses the second iteration of this survey, the 2015 National Food Hub Survey, which was collected by researchers at Michigan State University and The Wallace Center at Winrock International. This data set is used with permission as part of 
a wider collaborative project on food hubs with Michigan State University faculty under a grant from the USDA's National Institute of Food and Agriculture. Specifics about sampling methodology can be obtained by consulting Hardy and others (2016), the report of findings from the 2015 National Food Hub Survey. On the basis of best estimates of the total population of organizations that identify as food hubs, the 151 total responses in the 2015 National Food Hub Survey accounts for roughly one third of all food hubs in the United States.

The 2015 National Food Hub Survey elicits information from FHO managers in a number of organizational domains including structural and operational features of the firm, human resources, suppliers, product mixes, outbound marketing channels, community service offerings, mission and goals, perceptions about challenges and opportunities, and others. This makes the survey instrument exceptionally long; the instrument also suffers from poor design in terms of the logic, wording, and structure of data input in several of the key sections. It is unclear whether the survey was enumerated by research staff, with completion being left unguided and with few or no validation checks. This means that some data points that would ideally be included in the search for homeostatic property clusters (HPCs; for example, staffing and human resources available to the firm) are unretrieveable and precluded from use. Mitigating against these concerns is the uniqueness of a data set that is as comprehensive in scope and with a coverage rate of the population this large- positive elements that cannot be overstated.

Careful inspection and cleaning of the data set using deductive survey logic, however, yields an adequate data set without the need for imputation, a concern when performing cluster analysis. Given the nature of cluster analysis, cases are eligible for 
inclusion only if they have a valid observation for every variable that is an input to the clustering algorithm; cases with one or more missing elements are excluded from the analysis automatically, reducing the total sample size. To check validity, I ran an analysis of variance (ANOVA) to compare all variables of interest (clustering and validation) between in- and out-of-sample groups. Only one clustering variable showed any meaningful level of statistical significance — having a licensed shared-use kitchen ( $p=$ 0.089) - and two validation variables, both of which are correlated with each other, were significant—institutional sales $(p=0.061)$, business model $(p=0.036)$. Given the low $p$ value for the clustering variable and the relative unimportance of the other two as they are captured through other validation variables, we are not concerned about differences between in- and out-of-sample cases. Variables available in the data set and of interest in classificatory work are of mixed forms - some are continuous measures, others scaled (or scalable), and yet others categorical or dichotomous.

\subsection{Results}

I apply the methodological procedures outlined in Chapter 3 to the 2015 National Food Hub Survey data to search for HPCs among its cases and thereby identify candidate kinds to then be accepted or rejected as species of FHOs. The following subsections and associated tables and figures present the results for each of the stages of that method, thereby presenting a proof of concept for operationalizing the OSC. First, I discuss several key findings that drive the selection of variables for use in cluster analysis procedures and validation measures. This includes some theoretical motivations as well as quantitative results as part of the iterative selection process. Then I discuss the results of the iterative clustering procedures used to search for HPCs. I emphasize the results in the final round of cluster analysis, which yields the final postulated clustering solution-a 
preliminary set of kinds. I discuss the empirical results related to validation stages that use the kinds of the final postulated clustering solution (FPCS) as fixed factors (that is, membership in a kind or not). These include ANOVA, independent-samples $t$ test, and regressional analyses to determine the magnitude, direction, and ability to make inferences (statistical significance) about the effect of membership in a kind on strategic orientation/niche.

\subsubsection{Initial Variable Selection: Theoretical and Empirical Motivations}

Investigation begins by selecting variables that represent (a) the central morphology of the organizations, (b) the organization's niche within LRFSs, and (c) the firm's performance—overall and in terms increasing LRFS scale. In the initial phase, I used reasonable judgment on the basis of theoretical considerations and information gathered during qualitative research processes to select a preliminary information set for use in this inquiry. Theoretical considerations are important especially when considering morphological variables: as mentioned in Chapter 3's discussion of HPCs, our search is especially for variables that economic understanding would lead us to view as "sticky"that is those that are generally expected to be fixed within some intermediate time horizon. For outcome variables - those that represent the organizational niche of the firms-I look to items that are indicated by the literature discussed earlier as central strategic orientations of FHOs: customer bases in the form of marketing channels, product mixes, and the degree and methods for enacting a commitment to place (that is, enacting social embeddedness through community services).

Given the richness of topics covered in the 2015 National Food Hub Survey, many data points are reasonably eliminated out-of-hand as not pertinent to organizational form under the preceding definition of FHOs. Tables 4.1 and 4.2 describe this 
preliminary set. The first of these, Table 4.1, reports the 16 different informational realms used as morphological characteristics during the various rounds of cluster analysis (for more on these rounds, see section 5.3.2). One of these variables- total value of product moved (through the FHO) — was later reclassed as a performance variable and is used in Chapter 6's discussion of scale and ecologies. Table 4.2, meanwhile, reports variables that either (a) have been uses as strategic, performance, or exogenous elements to validate cluster solutions, or (b) were considered as morphological items but dismissed upon empirical investigation.

The Notes column in Table 4.2 provides the general reason for eliminating a variable from use before clustering procedures, usually because of concerns over data completeness or response validity. ${ }^{70}$ However, one can see in Table 4.1 that during clustering exploration a number of other morphological variables were eliminated in successive rounds. The reasons for this are varied, typically hinging on one or more of three reasons: (1) completeness across respondents reduced the effective sample size considerably so as to make information loss from dropped cases outweigh that from the omitted variables $^{71}$; (2) a variable has an approximate of an alternative form and the information-rich form is given preference; (3) correlation analysis showed that a variable is highly correlated with another. For (3), I conducted a correlation analysis with Pearson's correlation coefficients using SPSS, which automatically adjusts for binomial

\footnotetext{
${ }^{70}$ For example, data on staffing numbers across categories of employees and worker demographics were riddled with clear logical failings or missing values, most likely attributable to poor design of these survey elements and the lack of enumeration by a survey team member to ensure respondents could complete complex data table questions.

${ }^{71}$ For example, response rates on the expenses series were so low that the effective sample size reduces by nearly 25 percent in variable sets $E$ and $F$ compared with variable sets $G$ and $H$.
} 
variables versus continuous ones. When morphological variables showed high values for Pearson coefficients, I favored those with better theoretical grounding or better response rates for retention. Bilateral correlations with a coefficient greater than 0.40 or greater are reported in Tables 4.3 through 4.5.

Table 4.3 focuses on bilateral correlations of concern among morphological variables only. Observations from such analysis, combined with issues of completeness, motivated the eventual elimination from use in clustering of the expense series, supplier demographics and numbers, services offerings, and grant dependency. Table 4.4 provides correlation coefficients for bilateral relationships, where one variable is a morphological candidate and the other a performance, strategy, or explanatory variable. Related analysis further motivated elimination of expense and supplier data. Table 4.5 reports correlation coefficients among nonmorphological variables only. Items here are less important, except for in considering the final validation stage: regression analysis. Many of the results here are also unsurprising. For example, variables that are an item in a sum are expected to be highly correlated with the sum itself (such as a given community service with the total number of community service offerings). Some make economic sense, such as reliance on products that are highly perishable or of high value being positively correlated with total expenses or total revenues, respectively. The results provide preliminary evidence of a clustering phenomenon among community service types. Scatter plot matrices were conducted to identify potential clusterability of variables; results of this graphical approach did not unequivocally indicate clusterability in bilateral comparisons, however, increasing the importance of correlation analysis results. 


\subsubsection{Preliminary Clustering Models: Results From Iterative Rounds Exploring Variable Sets and Parameters}

Application of cluster analysis to determine groupings of instances on the basis of HPCs took place in two tranches. The first tranche was exploratory and focused on examining clustering parameters and variable sets. Tranche 2 included six variable sets. Results from tranche 1, as well as underlying statistical properties, guided the adoption of fixed parameters for the similarity and standardization techniques — squared Euclidean distance and 0-1 range, respectively - for all analyses in tranche 2 . These two parameters are preferable given their mathematical properties. Fixing the similarity measure to squared Euclidean distance across all linkage algorithms enables comparison across linkage approaches (given that Ward's method requires squared Euclidean distance) and helps identify outlier cases. In tranche 1 analyses, squared Euclidean distance models performed better than those with other distance measures at identifying groupings of cases in terms of the face validity of property clusters. Standardizing variables to the $0-1$ range is preferred for its statistical properties over other approaches such as $z$-scores, and also better fits this inquiry's use of mixed-scale data, including dichotomous variables.

In tranche 2, I examined eight variable sets in four rounds of clustering. During these rounds, various models were run and the quantitative and qualitative evaluations described in Chapter 4 were used. In each successive round, I dropped from consideration variable elements as morphological components in searching for parsimony, model congruence, and inclusivity of observations; these changes are tracked in the right half of Table 4.1. For each round, I performed an ANOVA in SPSS comparing in- and out-ofsample cases to determine the relatively validity of the effective sub-samples on a variable-by-variable basis; generally, results showed only a small number of variables 
with statistically significant differences in variances between in- and out-of-sample sets, and typically only those of marginal importance to the analysis. I do not report the various qualitative or quantitative measures (such as the cophenetic correlation coefficient, Calinski-Harabasz index, Akaike information criterion, and Bayesian information criterion), mean profiles, ANOVA, or independent-samples $t$ test results for preliminary clustering rounds here, as the amount of data is overwhelming. Between rounds, I placed emphasis on making improvements that addressed both theoretical and empirical concerns. This included examination of clustering parameters, such as variable standardization (where a standardizing to the range of 0 to 1 was preferred, especially given the morphological data contains multiple scales and dichotomous elements). Results are consistent across rounds in that average- and single-linkage solutions consistently exhibit higher CCCs than Ward's and complete.

The fourth and final round of cluster analysis compared two variable sets, E and F (see Table 4.1), using four agglomeration methods each for a total of eight models (see Table 4.6). The two variable sets compared in this final round were identical except for one difference: the first set included two variables measuring the total percent of distribution- and value addition-related services conducted by the food hub, whereas in the second set these were dropped and replaced with dichotomous variables indicating the presence of absence of five infrastructure elements. Those elements are bundles of physical assets relevant to the performance of the various distribution- and value addition-related services. Correlation analysis results (including those indicated in Tables 4.3 through 4.5 ) indicate that the physical assets are highly correlated with offering more 
services and that there may be clusterability among physical assets within the two services types.

Models that are based on these two variable sets are more parsimonious and improve on those from previous rounds in terms of the theoretical grounding for included properties as well as greater consistency of indicated partitioning solutions across the three measures of internal cohesion. These improvements come at only a small reduction in CCC for some models, and increases for others, over those in previous rounds. Both of the variable sets in the fourth round allowed for the inclusion of the same cases in the analysis; a total of 103 observations (68.2 percent of the total in the 2015 National Food Hub Survey data set) were included in the models, an improvement over earlier rounds. As a validity check, I conducted an ANOVA to compare variances of morphological and outcome variables between the in-sample and out-of-sample cases. More than 85 variables were examined in this manner. Table 4.7 reports results of these subsample validation procedures for this round. Only seven variables showed statistically significant $F$-values for ANOVA between in- and out-of-sample cases. Only one of these-offering transportation services to producers - is used as a morphological feature of the firms for clustering.

Overall, the eight models show fair to good recovery of the underlying proximities between cases by the cophenetic matrix of joining distances. It is important to note that these more parsimonious models show an improvement in $\mathrm{CCC}$ measures over preliminary models. Results are consistent across rounds in that Average and Single linkage consistently exhibit higher CCCs than Ward's and complete. Given the little agreement among the three internal clustering criteria about the appropriate number of 
partitions to draw across the various models, I turned to the adjusted Rand index matrix, consulting the congruencies between each of the partitioning solutions indicated as preferential by the Calinski-Harabasz index. This matrix is reported in the upper portion of Table 4.8 (the portion in white). These results indicate high congruence among cluster solutions using average and single linkage, something not surprising given that each of those four solutions (a) favors a very small number of clusters which (b) have a chaining structure as discussed earlier. Given this, we approach this result, and the average and single linkage methods overall, with caution; investigation of respective dendrograms shows that for this data set the elements (a) and (b) just noted are present.

By the nature of the $\mathrm{CCC}$, such a chaining structure predisposes a high correlation between the distance/similarity matrix and the cophenetic matrix. Acceptance of these models would immediately imply that there are no differentiable clusters of food hubs in the data set and thus a conclusion that no organizational species exist at this level (which may or may not lead to food hubs being accepted as a single taxonomic unit at the species level). Although this is possible, I am skeptical pending further investigation. In addition, the preference in the literature is for Ward's method in the recovery of clusters. This preference is supported by the connection between Ward's method under HCA and nonhierarchical $k$-means clustering.

\subsubsection{Final Postulated Cluster Solution}

After running the eight models identified, the Calinski-Harabasz index, Akaike information criterion, and Bayesian information criterion for each were calculated in addition to the cophenetic correlation coefficient used in Stage 1 decisions. Table 4.6 reports these results. Overall, the cophenetic matrix of joining distances for each of the eight models shows fair to good recovery of the underlying proximities between cases. 
However, these results, although more consistent (especially for variable set F), fail to provide unequivocal guidance as to a preferred clustering solution in terms of both linkage method and number of partitions, $k$. Thus, I turned to qualitative investigation of the mean profiles of the Calinski-Harabasz index-indicated partitions across models.

To accomplish this, I first considered the results of multidimensional scaling to map cluster orthographies into three- and two-dimensional space, visually inspecting the results for cohesion and separability. Normalized raw stress coefficients for multidimensional scaling (MDS) analyses are presented in Table 4.9. At three dimensions, these coefficients are below the preferred threshold of 0.05 . Thus, we can be relatively confident that these are acceptable reductions of the multivariate space. For two dimensions, we must use more caution. Given this, I consulted the visual representations of the Calinski-Harabasz index-indicated partitions mapped into the three-dimensional space using MDS coordinates. Graphics for the models using Ward's and complete linkage methods are presented in Figures 4.5 through 4.8. Visual separability of clusters in these figures is low, providing only partial guidance. In general, the Calinski-Harabasz index-indicated clustering solution of model $5, k=9$ shows better separability and cluster composition than other Calinski-Harabasz index-indicated solutions, relatively, followed by model $1(k=11)$, then by model $2(k=12)$.

However, because MDS visualizations for these models show dispersion, I consider other signals to guide decisions in the exploratory analysis of FHO HPCs. First, I consider mean profiles, to examine solutions for face validity. These results—not reported here as the data outputs are onerous—also indicated model 5, with readily interpretable separations in mean profiles. Of note is that model 2's Calinski-Harabasz 
index-indicated partitioning solution $(k=12)$ creates clusters with small memberships, making interpretation problematic. Given this, and the discussion earlier, models 5 and 1 are preferred. Both use Ward's method as the linking algorithm, but their underlying variable sets are different. Among the three internal clustering criteria examined, variable set $\mathrm{F}$ (underlying models 5 through 8 ) has greater agreement, as measured by ARI (see Table 4.8 for selected results), between Calinski-Harabasz index-indicated partitioning solutions across models. ${ }^{72}$ Furthermore, an appeal to economic theory supports adoption of this approach to organizational morphology; as noted earlier, the two services counts in variable set $\mathrm{E}$ are correlated with certain of the physical asset series which replaces them in variable set $\mathrm{F}$. The latter holds more detailed information about each case, and more closely mimics a simplistic understanding of "morphology."

Taking these statistical and qualitative features into account, I conclude that the variable set $\mathrm{F}$ is preferred to variable set $\mathrm{E}$. Model 5 is the preferred model on which to continue examination of the OSC in the context of FHOs. Therefore, I promote model 5, using variable set $\mathrm{F}$, as the preferred approach to identifying HPCs among the sample of FHOs. However, given the disagreement between the three measures of internal validity on the preferred number of partitions for model 5 , I pause for further consideration before promoting the $k=9$ solution out of hand as the FPCS. I investigate whether a reduction in the number of partitions is warranted, especially noting some of the observations made about the mean profiles of the $k=9$ partitioning solution.

One method for selecting partitions is the joining-height maximization rule discussed in Chapter 3. Table 4.10 reports the heights of the joining nodes, garnered from

\footnotetext{
${ }^{72}$ The average ARI in variable set $\mathrm{E}$ models is 0.197 , whereas for variable set $\mathrm{F}$ it is 0.214 .
} 
the cophenetic matrix, for the 11 partitioning solutions calculated during cluster analysis procedures. The partitioning solution of $k=4$ presents the greatest difference in joining heights, moving from the top of the dendrogram down. I set this as the minimum partition boundary for model 5, and the Calinski-Harabasz index-indicated $k=9$ as the maximum, to investigate comparative performance of partition solutions between them, searching for face validity, as well as the congruence between solutions using the ARI.

It is notable that examination of mean profiles across the joining of the clusters shows improvements in face validity at the $k=6$ solution. ANOVA's were conducted to compare $F$-statistic significance levels for morphological variables across partitioning solutions for model 5. The $p$ values for these results are shown in Table 4.11. There is relative stability in statistically significant differences for variation in the morphological variables. The six- and nine-cluster partitioning solutions have the same number of morphological properties with observed statistical significance of different group variances at or beyond the 10 percent level, with a trade-off between firm age and profit motivation. Both $k=6$ and $k=9$ exhibit the largest proportion of morphological properties with statistically significant differences in variances between groupings (12 of the 14 properties). The ARI between $k=6$ and $k=9$ solutions (reported in Table 4.8 ) is high, at 0.786 , indicating that there is substantial congruence between cluster membership. Compare this to 0.508 for $k=4$ and $k=9$ or 0.689 for $k=4$ and $k=6$. Taking these observations into account, I promote the six-partition solution of model 5 as the FPCS.

The mean profiles of the FPCS for morphological variables, along with the 95 percent confidence intervals, are reported graphically in Figure 4.9 as error-bar plots. 
Continuous variables are displayed as standardized, set to a range of 0 to 1 . The level of statistical significance for the group ANOVA $F$-statistic of each variable is reported using asterisks. ${ }^{73}$ Appendix Table A.1 reports the data itself. On the basis of the mean profiles for each cluster in the FPCS, I assign colloquial monikers that act as descriptive tags; these monikers, as well as sample frequencies and percentages are provided in Table 4.12. Figures 4.10 and 4.11 present information about the relationships between the candidate species. Figure 4.10 is the dendrogram of model 5 , annotated to show the positioning of each candidate species. This figure represents the hierarchical agglomeration of cases into groupings. It shows the structure of cluster joinings under the model's linking algorithm. Meanwhile, Figure 4.11 indicates the position of each candidate species' cases within a reduced-dimension space. Comparing the orthography of the candidate species in Figure 4.11 to the structure of their joining in Figure 4.10 provides a means of assessing the performance of the FPCS.

\subsubsection{Validation of the FPCS}

The initial step in validation is to determine whether the partitions in the FCPS have meaningful differences in outcomes and performance. Under my OSC framework, for a FPCS to be accepted as a set of organizational species, there must be meaningful differences in strategic orientations of the candidate clusters, which proxy the niche inhabited by the organizational population. For food hubs, three realms of strategic orientation are selected: (1) the product mix selected by the firms, (2) the marketing channel mix used by the firms to sell product, and (3) the intensity and mix of community services they offer as an expression of their social missions. Items 1 and 2 are measured

\footnotetext{
${ }^{73 *} \alpha=0.10(10 \%$ percent level $),{ }^{* *} \alpha=0.05$ (5 percent level), and ${ }^{* * *} \alpha=.01$ ( 1 percent level).
} 
through the "reliance" on products or marketing channels of the firms - that is as a percentage of sales attributable to a category. Item 3 is a series of dichotomous variables denoting the absence or presence of a community service being offered, as well as the total number of different services.

\subsubsection{ANOVA and Independent-Samples $t$ Test Results: Differences in Variances and Means}

Figures 4.12 and 4.13 report the mean profiles for the three strategy realms of the FPCS. Detailed data underlying these are provided in the appendix in Tables A.2 through A.4. Similar to analysis of morphological variables, ANOVA was conducted spanning partitioning solutions from $k=4$ to $k=9$ for all of the strategic outcome variables, for comparison. Tables 4.13 and 4.14 provide the $p$ values for the $F$-statistics.

For the FPCS, only one of the product categories — milk and other dairy products - shows a statistically significant difference in group variance, a departure from other partitioning solutions where processed produce, fresh produce, and meat and poultry have significant ANOVA $F$-statistics. That these are significant for the $k=5$ and $k=4$ partitions implies the combining of clusters drives the significance. The percentage of sales to DTR shows statistically significant difference consistently across partitioning solutions. Only at $k=9$ partitions do ANOVA results show statistically significant differences in group variances for either of the other three marketing channels. The FPCS also shows statistically significant differences in variance for six of the community service variables, including the total number offered. For this data set, where the number of observations overall is low let alone within groupings, the 95 percent confidence intervals for the means are wide for many variables and clusters. 
The overall strategic orientations of the prototypical (that is, mean) member for each species are summarized in the right column of Table 4.12, which are based on ANOVA results for the FPCS. Within product space, all FHO species rely on fresh produce as their primary offering, with the question being the degree to which they diversify into other products. As Figure 4.12 shows, this ranges between roughly 40 percent and 70 percent for the mean. In channel space, two thirds of the candidate species have direct-to-consumer sales as their primary channel, with the other one-third (clusters 1 and 6) holding DTR channels - primarily restaurants and small groceries—as their strategic orientation. Last, one candidate species (cluster 4) unambiguously performs far more community services than the others, whereas another (cluster 5) performs very few, and mostly those associated directly with off-loading excess product (donations to food banks) and stimulating market demand (educational programs with the public and with schools). The orthographies of these clusters in the dendrogram (Figure 4.10) are all immediately proximal in morphological similarities.

In addition to the overview results provided by the ANOVA results, pairwise comparisons of mean differences through independent-samples $t$ test procedures deepen our understanding of the differences in species and provide information on the magnitude and probability of error of bilateral group comparisons. Here, I examined two types of comparisons: the set of cases in a given species compared with all other cases (Tables 4.15 and 4.16), and species to species (Tables 4.17 through 4.19). In these five tables, only the mean differences are presented. Values in bold are those for which the mean difference could be determined to be statistically significant at or beyond the 10 percent level. Observations are only statistically significant results if $p$ values for $t$ statistics 
matched the conclusion of the associated $F$-statistic's $p$ value about the assumption of equal and different variances (that is, if the $F$-statistic was significant, we reject the null hypothesis that the variance are equal, and thus evaluate the $p$ value for the different variances $t$-statistic). Note also in these tables how to interpret the signs of mean differences: independent-samples $t$ test compares the reference set to the considered set, and thus a negative sign indicates that the considered set has a mean that value greater than the mean of the reference set. In Tables 4.15 and 4.16, the reference set is all other cases not of the considered species, whereas for Tables 4.17 through 4.19 the reference set is the species first listed in the column header and the considered set is the second species (that is, following "v").

In general, independent-samples $t$ test results bolster the observations made in Figures 4.12 and 4.13 about the relative positioning of the prototypical member for each species. Results show that there are a number of meaningful differences in means between the candidate species. In Table 4.15, covering single clusters compared with all other cases, we expect to have many statistically significant results because the variables reported here are morphological properties included in the search for HPCs; that search uses Ward's method, linking cases to minimize variance. Here, magnitudes are highly important to consider. For example, species 2 have a smaller mean age $(0.49$, in natural $\log$ terms) and warehouse space (8322.2 square feet) than does the average for all other FHO cases in the sample; we can conclude that there is a 90 percent chance that true values for both mean differences are at least as big if not bigger than the ones we observe.

Table 4.16 covers the strategic outcomes variables and exhibits fewer statistically significant results, although many are noteworthy. Consider, for example, that species 2 
and 3 have on average much fewer sales to restaurants and retailers $(20.16$ percent and 23.33 percent, respectively) and that species 5 has fewer sales ( 12.13 percent) to institutional buyers, compared with all other FHO cases not of those groups. Species 1 does more DTR sales and fewer DTC sales compared with others, whereas the reverse is true for species 2. Meanwhile, species 4 does much less in the way of fresh produce sales than do other species (nearly a quarter less, at 24.26) and offers many more different types of community services $(-2.73)$.

Species-to-species comparisons continue to provide evidence of how and to what degree the groupings of cases differ, on average. A prime example, from Table 4.17, is to consider the row of mean differences for DTR reliance, where a total of 5 bilateral pairs have statistically significant values, most of which have relatively large magnitudes. From this, species 1 has a mean reliance on DTR channels much larger than that of species 2 through 4 (and slightly largely, although without the same certainty, than the other two species). In contrast, species 3 does much less, on average, in terms of DTR sales than do species 5 and 6 . It is important to note that these species-to-species comparisons can guide the researcher in understanding the differences between groupings that may be considered for combination or separation under differing partitioning solutions. For example, species 1 and 6 only show statistically significant bilateral mean differences for three morphological variables: having retail space $(0.28)$, renting space to others $(-1.00)$, and total warehouse space $(-17,720.94)$. The differences in their strategies, without accounting for exogenous elements, are not overly consequential, with only four outcome variables showing statistically significant mean differences: coffee and 
tea ( 0.34 percent), nonfood items ( 0.72 percent), DTM (3.40 percent), and being engaged in the community service of paid employment for youth $(-0.30)$.

\subsubsection{Regression Analysis Results}

ANOVA and independent-samples $t$ test analyses can present only a part of the picture; they show comparative differences between species but do not account for factors exogenous to the morphological features of the firms. A robust consideration of organizational species requires additional investigation to account for such exogeneity in strategic orientations. Here, I adopt a regression analysis to accomplish this, estimating linear regressions using SPSS's multivariate generalized linear model functionality. I examine the set of dependent variables for strategy noted earlier.

In this analysis, cluster 1 - the candidate species I have termed “Average Joes"is used as the reference category for fixed-factor estimation because it presents the largest population. Similarly, the variable used to represent geographic region has the region with the largest population in the sample as the reference category. This variable is the statistical regions used by the USDA's National Agricultural Statistical Service in the 2015 Local Foods Marketing Practices Survey. I select this as the regional variable because it is denoted for the same year as the 2015 National Food Hub Survey and is closely tied to data on LRFSs. Geographic information more detailed than the state of the FHO was not provided in the data set shared with the research team to protect disclosure standards of data collectors.

Regression coefficients, $p$ values, and adjusted $R^{2}$ for each of the strategic orientation variable regressions are reported in Tables 4.20 through 4.22. Unfortunately, because of data completeness in the 2015 National Food Hub Survey, few variables are ideal for inclusion as instruments in these regression estimations; more detailed 
regressions in terms of explanatory variables lead to dramatic losses in total observations included. Regressions 1 and 2 consist of 94 observations, whereas Regression 3 consists of 91. These models account for species effects, regional effects, and then, a proxy for managerial experience (the age of the most senior manager). Only in rare cases are regional effect coefficients statistically significant across the strategy measures and in most cases this is only weakly so. The exception: these results present evidence that being a $\mathrm{FHO}$ in region ${ }^{74} 5$ (compared with region ${ }^{75} 2$ ) has a large and significant positive effect on percent of sales to DTI channels and on product positioning in processed produce. Manager age similarly shows little contribution to FHOs' strategies, with only two instances of statistical significance (milk and other dairy; eggs) both of which have negative signs but low magnitudes. The inclusion of this variable in the regression analysis appears to shift magnitudes for both intercepts and coefficients. This may be due to the three-observation loss that comes with its inclusion.

Crucial to validating the FPCS as species is that the patterns seen in the ANOVA and independent-samples $t$ test results for the candidate species generally hold when accounting for exogenous factors; coefficient signs and magnitudes are consistent, as are areas where conclusions have strong support as evidenced by $p$ values. These elements directly relate to the OSC developed and proposed earlier in this work, with its emphasis on niches, which I measure through strategic orientations. Species effects are most pronounced in the community services orientation of the populations, the two leading marketing channel orientations (DTC and DTR), and the top four product orientations

\footnotetext{
${ }^{74}$ Region 5 includes Alabama, Arkansas, Louisiana, Mississippi, Oklahoma, and Texas.

${ }^{75}$ Region 2 includes Connecticut, Delaware, Maine, Maryland, Massachusetts, New Hampshire, New Jersey, New York, Pennsylvania, Rhode Island, Vermont, and the District of Columbia.
} 
(Fresh produce, processed produce, meat and poultry, and milk and other dairy products). These results are signals, among a wider array, that help decide whether the candidate species proposed here are worthy of acceptance or rejection as differentiated organizational forms and thus as distinct populations.

\subsection{Discussion}

The design and illustration of the OSC highlight the issues in categorization of organizational types. The conceptual issues include the choice of attributes of the organizations that become the clustering variables. Is there sufficient variation within these attributes to obtain distinct species? Is there a sufficient number of attributes to describe a structure space large enough to discern the centroids and the boundaries of species clusters without confounding overlaps? Are these attributes theoretically interesting or merely convenient? The methods issues include using statistical tools, visualization, and theoretically meaningful decision rules to validate one of the calculated cluster solutions as the final proposed solution. Also, are there meaningful variables on firm structure, behavior, or performance that have not been used to define the clusters that can be used in validation? That is, do we avoid using a technique (cluster analysis) that seeks to maximize distance between the centroids and then using the same variables in ANOVA to show that the clusters are different? In the earlier presentation of the food hubs example, these issues are illustrated as a series of iterative steps to bring alternative validation analyses to bear on candidate species structures in the data.

The results I present for food hubs show the nuanced nature of the process. A philosophical question arises: how different is different enough? In an earlier section, I noted the orthography of the candidate species. Specifically, attention focuses on two alternative pairings: cluster 6 paired to cluster 1 (pair A) and cluster 2 paired with cluster 
5 (pair B). Morphologically, pair A is separated by three elements: total warehouse space, offering rental space to other businesses, and having retail space. In strategy space, the two clusters differ marginally. Pair B shows statistically significant mean differences on six morphological variables, with magnitudes of these differences being substantial. Only one strategy variable shows unambiguously significant mean differences: nonfood sales. Strategies are similar within pairings. Pair A focuses on the DTR-led channel strategy supplemented by DTC, primarily selling fresh produce and offering the average number of community services. Pair B focus on a DTC-led channel strategy supplemented by DTR. However, cluster 5 supplements with DTM more than any other candidate species.

To draw the line between these organizational forms, I return to the regression analyses. When accounting for exogenous factors, cluster 6 has statistically significant coefficients in only one strategic variable: percentage of sales from fish products. In contrast, both clusters of pair B consistently show statistically significant coefficients across all regressions for both DTC (positive sign) and DTR (negative sign) sales; substantial magnitudes for DTI sales and total community services (both negative). Adopting the $k=5$ solution is appropriate, where clusters 1 and 6 are combined into a single organizational form. Given the empirical results, clusters 2 and 5 should be treated as distinct species.

However, qualitative data collected on background for this investigation gives me pause in respecifying the FPCS to $k=5$ for now. That background indicates that the differentiating factor between cluster 6 (Traditional Produce Warehousers) and cluster 1 (Average Joes) may arise in the origin of the former set; a number of qualitative accounts of firms had been operating in conventional food systems moving into the LRFS space. 
Such pathways are an important element of speciation, an evolutionary matter that is not captured in the phenetic approach taken here.

\subsection{Concluding Remarks}

Considering the results presented herein, it is warranted to accept the theorized taxa resulting from the cluster solution as organizational species. These groupings have face validity as kinds with HPCs that appeal not only to intuition but also to received theory of organizational economics. Qualitative analysis for validation provides evidence for drawing an ontological line between clusters 1 and 6 . We see an interesting question that cannot be answered with the extant data. The taxon Traditional Produce Warehousers may be a species born of mimicry (of century-old warehousing niches in industrial food systems) or they may be a "genetic offshoot" of extant warehouses that have the plasticity to serve the niche in these alternative local food systems. Much of the quantitative findings presented here triangulate well with this qualitative work. The biggest surprise in the quantitative analysis was the uniqueness of cooperative food hubs, which may mimic the cooperative form that is rampant in production agriculture and rural life. Cooperatives have consistently been identified within the organizational economics literature as a response to thin markets. Although my work here cannot prove causation, it hints that in food systems where promoting access to local meat and poultry is a priority, stimulating a cooperative-form food hub may help to overcome a lack of proper processing facilities.

New organizational forms (1) arise at the intersection of evolutionary processes and ecological structure and (2) develop with environmental changes in the sector. Social movements - such as the local foods movement, but also allied movements of alterity such as farm-to-table restaurants, Slow Food, organic production, civic agriculture, and sustainability_provide "strategic space" for establishing new forms. Weber, Heinze, and 
DeSoucey (2008) identified this phenomenon for the subsector that linked consumers to grass-fed livestock. In my study of food hubs, nonconstitutive attributes of the firms matter. Moreover, many of these attributes originate from other organizational forms that serve local and regional food systems, such as farmers' markets and communitysupported agriculture groups — which tie consumers directly to local producers. Some of the extant food hubs have evolved from farmers markets and community-supported agriculture groups; other de novo food hubs have mimicked the attributes, seeing them as essential to identifying a HPC that has meaning for other organizations in food systems. 


\subsection{Tables and Figures}

\section{Figure 4.1. Defining Characteristics of a Regional Food Hub}

\section{Defining Characteristics of a Regional Food Hub}

Regional food hubs are defined less by a particular business or legal structure, and more by how their functions and outcomes affect producers and the wider communities they serve. Defining characteristics of a regional food hub include:

- Carries out or coordinates the aggregation, distribution, and marketing of primarily locally/regionally produced foods from multiple producers to multiple markets.

- Considers producers as valued business partners instead of interchangeable suppliers and is committed to buying from small to mid-sized local producers whenever possible.

- Works closely with producers, particularly small-scale operations, to ensure they can meet buyer requirements by either providing technical assistance or findings partners that can provide this technical assistance.

- Uses product differentiation strategies to ensure that producers get a good price for their products. Examples of product differentiation strategies include identity preservation (knowing who produced it and where it comes from), group branding, specialty product attributes (such as heirloom or unusual varieties), and sustainable production practices (such as certified organic, minimum pesticides, or "naturally" grown or raised).

- Aims to be financially viable while also having positive economic, social, and environmental impacts within their communities, as demonstrated by carrying out certain production, community, or environmental services and activities.

Source: Reproduced from Barham and others 2012, a work in the public domain. 
Figure 4.2 Common Conceptual Model of Sustainability

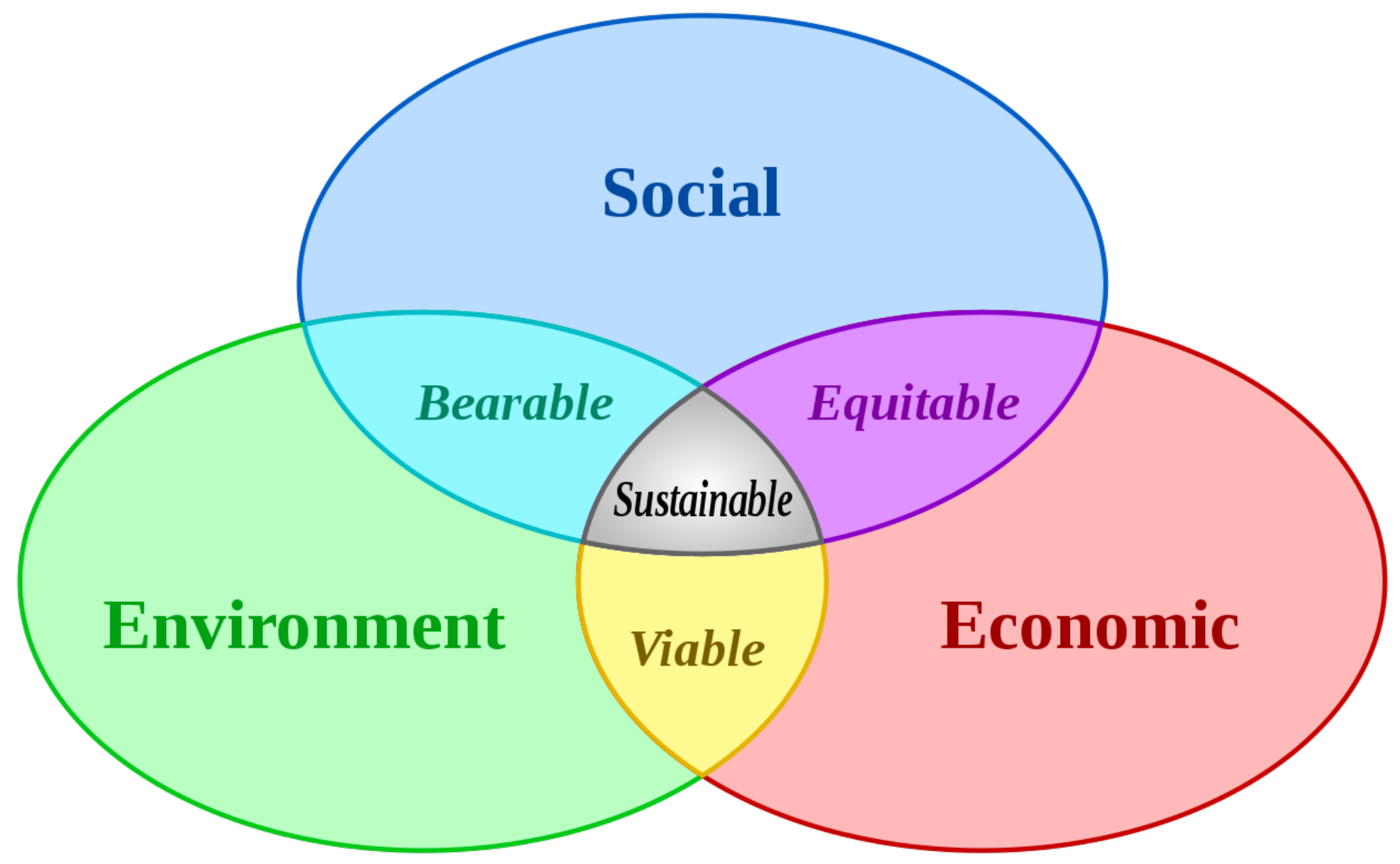


Figure 4.3 Legal Structures of Food Hub Organizations

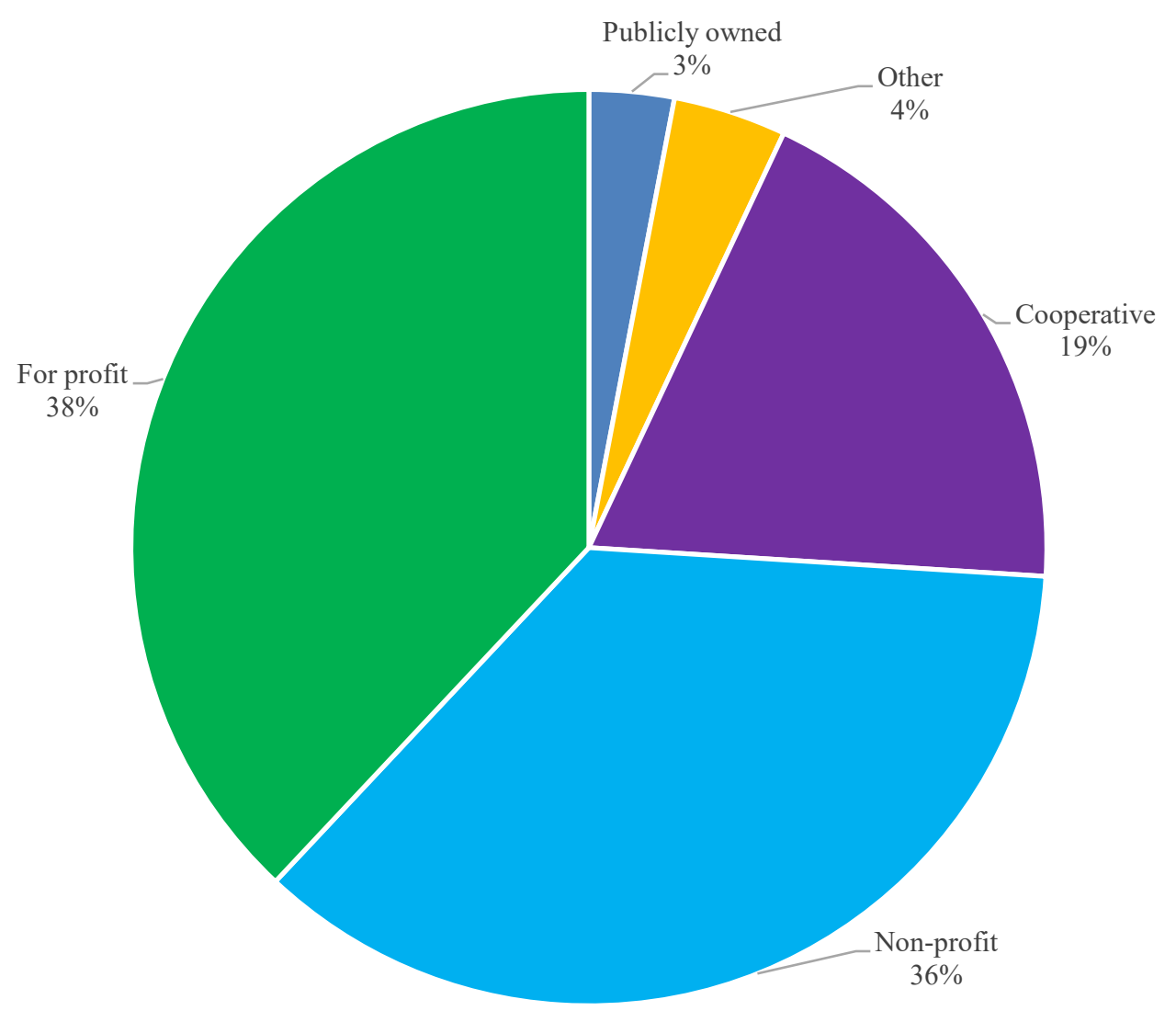

Source: Author's redrawing of Figure 3 from Hardy and others (2016, p. 11)

Note: Reported $n=151$. 
Figure 4.4 Percentage of Food Hub Organizations Procuring Products From Different Sources

Non-food businesses

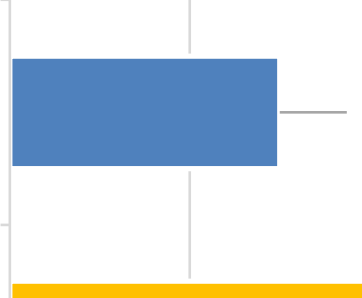

Food hub's operations

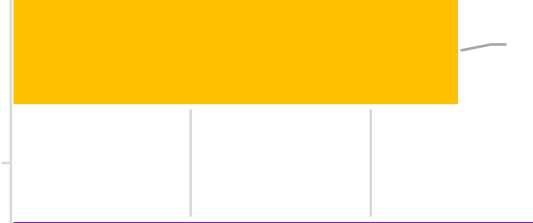

Food Distributors

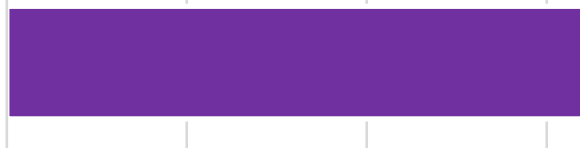

$-32 \%$

Food Processor
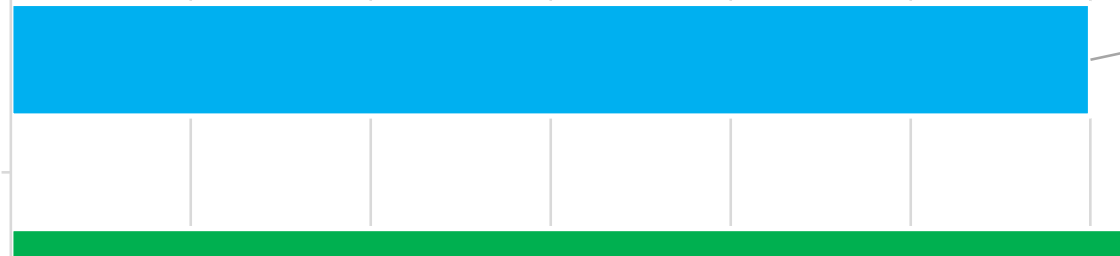

Farms and ranche

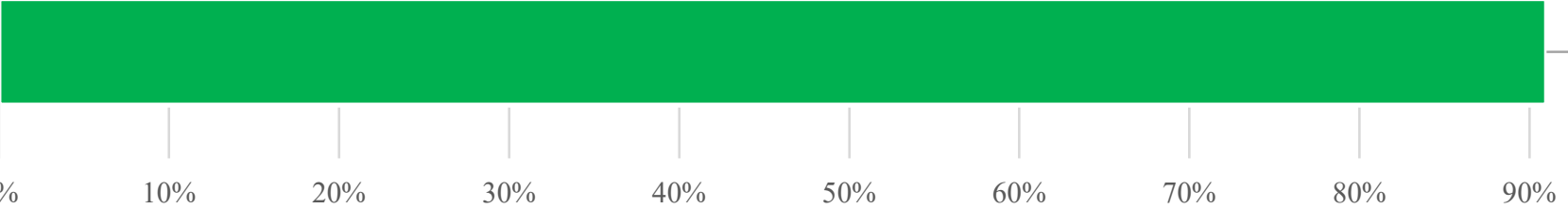

Source: Author's redrawing of Figure 8 from Hardy and others (2016, p. 18)

Note: Reported $n=111$ 
Table 4.1. Summary of Variable Sets in Interative Cluster Analysis Rounds

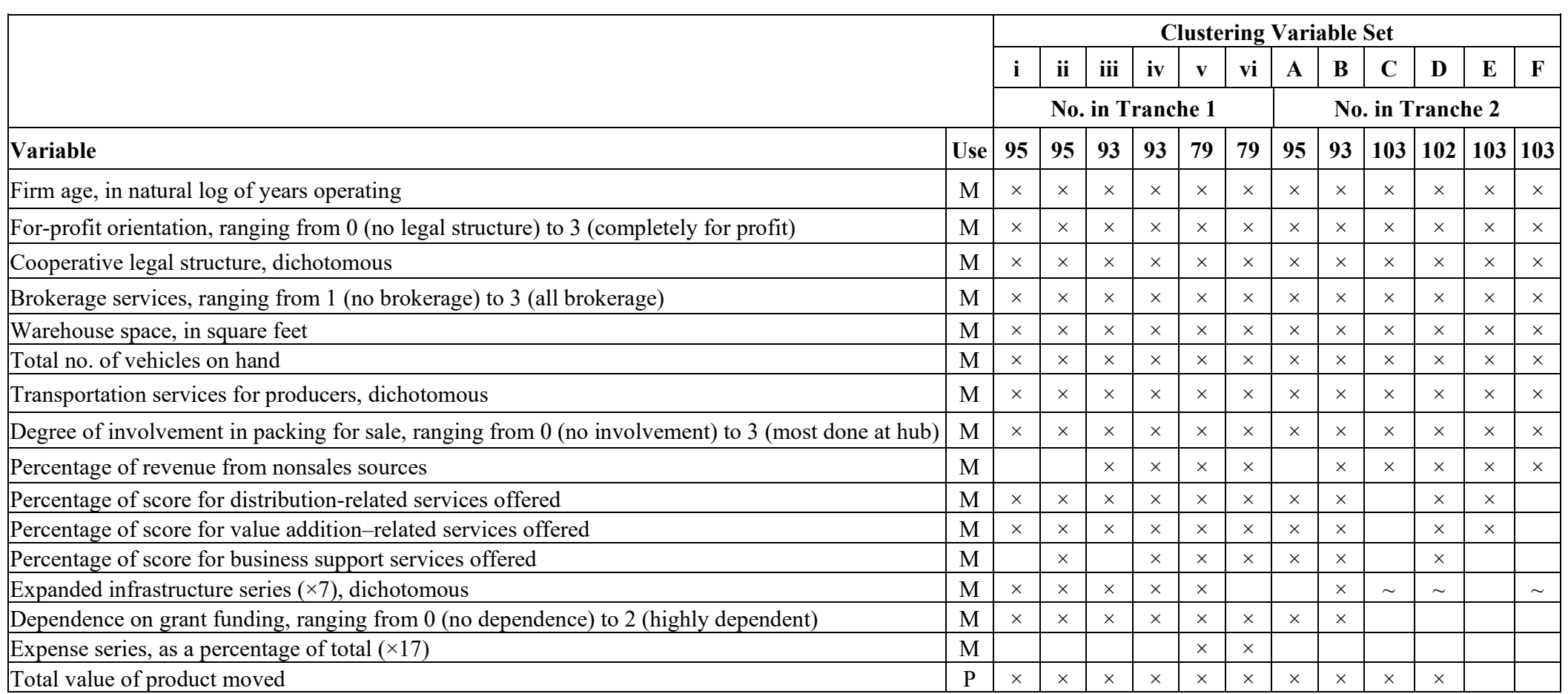

Source: Author.

Note: The "No. in Trache" columns represent the total number of cases (organizations) with complete data for that variable set and used for cluster analysis. $\times$ denotes the variable is included in the set and is used for clustering procedures. $\sim$ denotes partial inclusion of series elements, with some elements of the series omitted. $\mathrm{P}=$ performance; and $\mathrm{M}=$ morphological. 
Table 4.2. Summary of Additional Variables Considered for Use in Applying the Organizational Species Concept to Food Hub Organizations

\begin{tabular}{|c|c|c|}
\hline Variable & Use & Notes \\
\hline Total value of product moved & $\mathrm{P}$ & \\
\hline Profit & $\mathrm{P}$ & \\
\hline Total revenue & $\mathrm{P}$ & \\
\hline Gross product sales & $\mathrm{P}$ & Total value of product moved plus brokerage values \\
\hline Product reliance (percentage of sales to product category) & $\mathrm{S}$ & \\
\hline Channel reliance (percentage of sales to marketing channels) & $\mathrm{S}$ & \\
\hline Expanded community service series & $\mathrm{S}$ & \\
\hline Total no. of different community service types & $\mathrm{S}$ & \\
\hline Business model: dichotomous $(\times 2)$ & $\mathrm{S}$ & Information contained in channel reliance \\
\hline Business model: 3-point scale & $\mathrm{S}$ & Information contained in channel reliance \\
\hline Vendors type series & $\mathrm{S}$ & \\
\hline Manager age & $\mathrm{E}$ & \\
\hline Total expenses & E & Completeness issues \\
\hline Manager experience in key hub fields series & $\mathrm{E}$ & Completeness issues \\
\hline State in which food hub organization is located & $\mathrm{E}$ & Transformed into Local Food Marketing Practices Survey regions \\
\hline Total no. of vendors & M & Completeness issues \\
\hline Percentage of vendors who are farms and ranches & $\mathrm{M}$ & Completeness issues; upstream connection \\
\hline $\begin{array}{l}\text { Percentage of vendors who are minority (female; person of color) or beginning } \\
\text { farmers and ranchers }\end{array}$ & M & Completeness issues; upstream connection \\
\hline Distance radius of majority of customers & $\mathrm{M}$ & Completeness issues \\
\hline Expanded services series: distribution, business, and value addition $(\times 14)$ & M & Information contained in sums \\
\hline Mission series $(\times 11)$ & M & Completeness issues \\
\hline Startup funds sources series $(\times 15)$ & M & Completeness issues \\
\hline Staffing numbers & M & Validation issues \\
\hline Expense series, as a percentage of total $(\times 17)$ & M & Completeness issues \\
\hline
\end{tabular}

Source: Author.

Note: $\mathrm{P}=$ performance; $\mathrm{S}=$ strategic orientation; $\mathrm{E}=$ exogenous; and $\mathrm{M}=$ morphological. 
Table 4.3. Selected Results of the Correlation Analysis: Morphological Variable Comparisons Only

\begin{tabular}{|c|c|c|}
\hline \multirow{2}{*}{$\begin{array}{c}\text { Pearson } \\
\text { Correlation } \\
\text { Coefficient }\end{array}$} & \multicolumn{2}{|c|}{ Variable Pairing in Correlation Analysis } \\
\hline & Variable A & Variable B \\
\hline 0.922 & Total no. of suppliers & Number of delivery vehicles \\
\hline 0.787 & Percentage of suppliers: farms or ranches & Percentage of expenses: gasoline and tolls \\
\hline 0.598 & Number of delivery vehicles & Total warehouse space \\
\hline 0.595 & Total no. of suppliers & Total warehouse space \\
\hline 0.577 & Percentage of expenses: all types of insurance & Percentage of expenses: packaging equipment and supplies \\
\hline 0.568 & Percentage of suppliers: farms or ranches & Percentage of expenses: other administrative expenses \\
\hline-0.564 & Percentage of expenses: product purchases & Percentage of expenses: employee salary and benefits \\
\hline 0.543 & Processing facilities & Value-addition services percentage score \\
\hline 0.48 & Percentage of expenses: consulting services & Percentage of expenses: advertising and promotional materials \\
\hline 0.478 & Percentage of suppliers: farms or ranches & Percentage of expenses: credit card and bank service charges \\
\hline 0.468 & Shared use kitchen & Processing facilities \\
\hline-0.459 & Profit motivated legal status & Grant dependency rating \\
\hline 0.454 & Percentage of expenses: payments toward automotive equipment & Retail space for the hub \\
\hline 0.45 & Percentage of expenses: utilities & Percentage of expenses: repair and maintenance \\
\hline-0.446 & Percentage of expenses: product purchases & Percentage of expenses: other \\
\hline-0.424 & Percentage of suppliers: in operation less than 10 years & Geographic radius containing majority of customers \\
\hline 0.407 & Business services percentage score & Distribution services percentage score \\
\hline 0.400 & Grant dependency rating & Percentage of revenue attributed to sources other than sales \\
\hline
\end{tabular}

Source: Author's calculations.

Note: Coefficients for all listed pairings are statistically significant at or beyond the 5 percent level. 
Table 4.4. Selected Results of the Correlation Analysis: Morphological Variables Compared With Outcome Variables

\begin{tabular}{|c|c|c|}
\hline \multirow{2}{*}{$\begin{array}{c}\text { Pearson } \\
\text { Correlation } \\
\text { Coefficient } \\
\end{array}$} & \multicolumn{2}{|c|}{ Variable Pairing in Correlation Analysis } \\
\hline & Variable A & Variable B \\
\hline 0.899 & Total expenses & Number of delivery vehicles \\
\hline 0.899 & Total no. of suppliers & Product reliance: fish \\
\hline 0.885 & Total no. of suppliers & Total expenses \\
\hline 0.817 & Total no. of suppliers & Total value of product moved \\
\hline 0.817 & Total no. of suppliers & Gross product sales \\
\hline 0.789 & Total no. of suppliers & Total revenue \\
\hline 0.768 & Product reliance: fish & Number of delivery vehicles \\
\hline 0.740 & Profit & Percentage of expenses: other administrative \\
\hline 0.634 & Total expenses & Total warehouse space \\
\hline 0.559 & Total value of product moved & Percentage of expenses: other administrative \\
\hline 0.558 & Gross product sales & Percentage of expenses: other administrative \\
\hline 0.548 & Total revenue & Percentage of expenses: other administrative \\
\hline 0.494 & Product reliance: coffee and tea & Percentage of expenses: gasoline and tolls \\
\hline 0.476 & Geographic radius containing majority of customers & Channel reliance: direct to intermediaries \\
\hline 0.470 & Total revenue & Number of delivery vehicles \\
\hline 0.462 & Percentage of suppliers: farms or ranches & Product reliance: coffee and tea \\
\hline 0.450 & Total value of product moved & Number of delivery vehicles \\
\hline 0.450 & Gross product sales & Number of delivery vehicles \\
\hline 0.436 & Percentage of suppliers: farms or ranches & Health screenings \\
\hline 0.433 & Product reliance: fish & Total warehouse space \\
\hline 0.418 & Percentage of suppliers: farms or ranches & Product reliance: milk and other dairy \\
\hline 0.411 & Geographic radius containing majority of customers & Product reliance: processed produce \\
\hline
\end{tabular}

Source: Author's calculations.

Note: Coefficients for all listed pairings are statistically significant at or beyond the 5 percent level. 
Table 4.5. Selected Results of the Correlation Analysis: Outcome Variable Comparisons Only

\begin{tabular}{|c|c|c|}
\hline \multirow{2}{*}{$\begin{array}{c}\text { Pearson } \\
\text { Correlation } \\
\text { Coefficient } \\
\end{array}$} & \multicolumn{2}{|c|}{ Variable Pairing in Correlation Analysis } \\
\hline & Variable A & Variable B \\
\hline 0.996 & Total value of product moved & Total revenue \\
\hline 0.996 & Total revenue & Gross product sales \\
\hline 0.832 & Product reliance: fish & Total expenses \\
\hline 0.792 & Total value of product moved & Profit \\
\hline 0.792 & Gross product sales & Profit \\
\hline 0.781 & Total revenue & Profit \\
\hline-0.747 & Channel reliance: DTC & Channel reliance: DTR \\
\hline 0.653 & Total expenses & Total revenue \\
\hline 0.653 & Accepting SNAP benefits & Total no. of community service types offered \\
\hline 0.635 & Total value of product moved & Total expenses \\
\hline 0.635 & Total expenses & Gross product sales \\
\hline 0.619 & Product reliance: other value added & Channel reliance: unclassified \\
\hline-0.603 & Product reliance: fresh produce & Product reliance: meat \\
\hline 0.586 & Nutrition or cooking education & Total no. of community service types offered \\
\hline 0.556 & Education for programs in community or school gardening & Total no. of community service types offered \\
\hline 0.552 & Accepting SNAP benefits & Matching programs for SNAP benefits \\
\hline 0.531 & Paid employment opportunities for youth & Total no. of community service types offered \\
\hline 0.529 & Education about community and food systems issues & Total no. of community service types offered \\
\hline 0.521 & Accepting WIC or FMNP benefits & Total no. of community service types offered \\
\hline 0.515 & Matching programs for SNAP benefits & Total no. of community service types offered \\
\hline 0.515 & Subsidized farm shares & Total no. of community service types offered \\
\hline 0.476 & Product reliance: coffee and tea & Product reliance: nonfood \\
\hline 0.468 & Accepting SNAP benefits & Subsidized farm shares \\
\hline 0.456 & Total value of product moved & Product reliance: fish \\
\hline 0.455 & Product reliance: fish & Gross product sales \\
\hline 0.449 & Product reliance: fish & Total revenue \\
\hline 0.443 & Operating a mobile market & Total no. of community service types offered \\
\hline 0.435 & Accepting WIC or FMNP benefits & Health screenings \\
\hline 0.417 & Accepting SNAP benefits & Accepting WIC or FMNP benefits \\
\hline 0.413 & Matching programs for SNAP benefits & Subsidized farm shares \\
\hline 0.409 & Other community services or activities & Total no. of community service types offered \\
\hline
\end{tabular}

Source: Author's calculations.

Note: DTC = direct to consumers; DTR = direct to retailers and restaurants; FMNP = Farmers Market Nutrition Program; SNAP = Supplemental Nutrition Assistance Program;

WIC $=$ Women Infants and Children (program). Coefficients for all listed pairings are statistically significant at or beyond the 5 percent level. 
Table 4.6. Statistical Indexes of Model Performance for Variable Sets E and F

\begin{tabular}{|c|c|c|c|c|c|c|c|c|c|c|c|c|}
\hline & \multicolumn{12}{|c|}{ Variable Set E } \\
\hline Model & \multicolumn{3}{|c|}{1} & \multicolumn{3}{|c|}{2} & \multicolumn{3}{|c|}{3} & \multicolumn{3}{|c|}{4} \\
\hline Method & \multicolumn{3}{|c|}{ Ward's } & \multicolumn{3}{|c|}{ Complete } & \multicolumn{3}{|c|}{ Average } & \multicolumn{3}{|c|}{ Single } \\
\hline $\mathrm{CCC}$ & \multicolumn{3}{|c|}{0.596} & \multicolumn{3}{|c|}{0.649} & \multicolumn{3}{|c|}{0.753} & \multicolumn{3}{|c|}{0.694} \\
\hline Partition & CHI & AIC & BIC & CHI & AIC & BIC & CHI & AIC & BIC & CHI & AIC & BIC \\
\hline 2 & 0.08 & 2387.44 & 2492.82 & 0.20 & 2445.14 & 2550.53 & 29.89 & 2493.94 & 2599.33 & 63.95 & 2496.75 & 2602.14 \\
\hline 3 & 0.06 & 2340.25 & 2498.33 & 0.35 & 2435.69 & 2593.77 & 32.08 & 2519.03 & 2677.11 & 31.67 & 2525.61 & 2683.69 \\
\hline 4 & 0.48 & 2325.41 & 2536.19 & 0.31 & 2408.62 & 2619.39 & 22.70 & 2422.86 & 2633.63 & 21.18 & 2547.80 & 2758.58 \\
\hline 5 & 0.68 & 2333.61 & 2597.08 & 0.85 & 2401.98 & 2665.46 & 29.82 & 2432.10 & 2695.57 & 15.78 & 2575.95 & 2839.42 \\
\hline 6 & 0.54 & 2333.35 & 2649.52 & 0.84 & 2370.08 & 2686.25 & 23.62 & 2460.70 & 2776.87 & 12.67 & 2547.44 & 2863.61 \\
\hline 7 & 1.23 & 2337.85 & 2706.72 & 11.18 & 2385.07 & 2753.94 & 19.49 & 2406.67 & 2775.53 & 18.78 & 2554.59 & 2923.45 \\
\hline 8 & 1.04 & 2357.56 & 2779.11 & 11.56 & 2376.61 & 2798.16 & 16.53 & 2418.84 & 2840.40 & 15.94 & 2491.27 & 2912.82 \\
\hline 9 & 0.90 & 2375.62 & 2849.87 & 10.04 & 2398.14 & 2872.39 & 14.38 & 2406.16 & 2880.41 & 13.81 & 2516.67 & 2990.92 \\
\hline 10 & 3.64 & 2388.65 & 2915.60 & 9.07 & 2422.66 & 2949.60 & 12.69 & 2418.75 & 2945.69 & 12.63 & 2477.30 & 3004.24 \\
\hline 11 & 4.10 & 2400.78 & 2980.42 & 10.22 & 2444.96 & 3024.61 & 11.31 & 2451.69 & 3031.33 & 14.13 & 2508.15 & 3087.79 \\
\hline \multirow[t]{2}{*}{12} & 3.69 & 2423.13 & 3055.47 & 12.12 & 2448.87 & 3081.20 & 13.50 & 2454.55 & 3086.88 & 12.72 & 2529.21 & 3161.54 \\
\hline & \multicolumn{12}{|c|}{ Variable Set F } \\
\hline Model & \multicolumn{3}{|c|}{5} & \multicolumn{3}{|c|}{6} & \multicolumn{3}{|c|}{7} & \multicolumn{3}{|c|}{8} \\
\hline Method & \multirow{2}{*}{\multicolumn{3}{|c|}{$\begin{array}{c}\text { Ward's } \\
0.561\end{array}$}} & \multicolumn{3}{|c|}{ Complete } & \multicolumn{3}{|c|}{ Average } & \multicolumn{3}{|c|}{ Single } \\
\hline $\mathrm{CCC}$ & & & & \multicolumn{3}{|c|}{0.565} & \multicolumn{3}{|c|}{0.752} & \multicolumn{3}{|c|}{0.714} \\
\hline Partition & CHI & AIC & BIC & CHI & AIC & BIC & CHI & AIC & BIC & CHI & AIC & BIC \\
\hline 2 & 2.52 & 3427.65 & 3538.31 & 3.48 & 3425.90 & 3536.56 & 11.89 & 3471.29 & 3581.94 & 22.19 & 3483.19 & 3593.85 \\
\hline 3 & 2.22 & 3346.05 & 3512.04 & 6.22 & 3420.00 & 3585.99 & 6.16 & 3397.02 & 3563.01 & 11.08 & 3502.04 & 3668.03 \\
\hline 4 & 1.85 & 3309.29 & 3530.61 & 4.57 & 3344.34 & 3565.66 & 4.20 & 3417.28 & 3638.60 & 7.46 & 3517.64 & 3738.95 \\
\hline 5 & 1.48 & 3282.72 & 3559.37 & 3.79 & 3340.22 & 3616.86 & 3.12 & 3438.16 & 3714.81 & 15.82 & 3549.09 & 3825.74 \\
\hline 6 & 2.46 & 3225.17 & 3557.14 & 3.02 & 3347.15 & 3679.13 & 5.59 & 3412.27 & 3744.25 & 12.59 & 3576.17 & 3908.15 \\
\hline 7 & 4.16 & 3215.10 & 3602.41 & 2.51 & 3314.65 & 3701.96 & 4.62 & 3432.17 & 3819.47 & 10.41 & 3599.85 & 3987.16 \\
\hline 8 & 3.54 & 3212.07 & 3654.70 & 2.34 & 3322.23 & 3764.86 & 3.99 & 3429.15 & 3871.78 & 8.88 & 3609.93 & 4052.56 \\
\hline 9 & 4.58 & 3211.40 & 3709.37 & 2.12 & 3329.57 & 3827.53 & 3.45 & 3450.48 & 3948.44 & 7.81 & 3568.44 & 4066.40 \\
\hline 10 & 4.07 & 3220.23 & 3773.52 & 1.95 & 3315.06 & 3868.35 & 4.49 & 3481.23 & 4034.52 & 9.64 & 3587.69 & 4140.99 \\
\hline 11 & 3.62 & 3229.13 & 3837.75 & 1.86 & 3295.71 & 3904.33 & 4.09 & 3409.73 & 4018.35 & 15.62 & 3594.42 & 4203.04 \\
\hline 12 & 3.27 & 3243.90 & 3907.85 & 1.70 & 3297.22 & 3961.17 & 3.73 & 3442.58 & 4106.53 & 14.08 & 3617.24 & 4281.19 \\
\hline
\end{tabular}

Source: Author's calculations.

Note: Cells highlighted in green signify critical value for measure (maximum for $\mathrm{CHI}$, minimums for $\mathrm{AIC}$ and $\mathrm{BIC}$ ). $\mathrm{AIC}=\mathrm{Akaike}$ information criterion; $\mathrm{BIC}=\mathrm{Bayesian}$ information criterion; $\mathrm{CCC}=$ cophenetic correlation coefficient; $\mathrm{CHI}=$ Calinski-Harabasz index. 
Table 4.7. Variables With Statistically Significant Mean Differences Between In-Sample and Out-of-Sample Cases

\begin{tabular}{|c|c|c|c|c|c|c|c|}
\hline \multirow[b]{2}{*}{ Variable } & \multirow[b]{2}{*}{$N$} & \multirow[b]{2}{*}{ Mean } & \multirow{2}{*}{$\begin{array}{l}\text { Standard } \\
\text { Deviation }\end{array}$} & \multirow{2}{*}{$\begin{array}{c}\text { Standard } \\
\text { Error }\end{array}$} & \multicolumn{2}{|c|}{$95 \% \mathrm{CI}$} & \multirow[b]{2}{*}{$P$ Value } \\
\hline & & & & & $\begin{array}{l}\text { Lower } \\
\text { Bound }\end{array}$ & $\begin{array}{l}\text { Upper } \\
\text { Bound }\end{array}$ & \\
\hline Offer transport service to producers & & & & & & & \\
\hline Out & 40 & 0.55 & 0.504 & 0.080 & 0.39 & 0.71 & \multirow{3}{*}{0.015} \\
\hline In & 103 & 0.76 & 0.431 & 0.042 & 0.67 & 0.84 & \\
\hline Total & 143 & 0.70 & 0.460 & 0.038 & 0.62 & 0.78 & \\
\hline Product storage facility & & & & & & & \\
\hline Out & 40 & 0.60 & 0.496 & 0.078 & 0.44 & 0.76 & \multirow{3}{*}{0.007} \\
\hline In & 103 & 0.82 & 0.390 & 0.038 & 0.74 & 0.89 & \\
\hline Total & 143 & 0.76 & 0.431 & 0.036 & 0.68 & 0.83 & \\
\hline Percentage of expenses to credit card and bank service charges & & & & & & & \\
\hline Out & 8 & 0.07 & 0.15 & 0.05 & -0.06 & 0.19 & \multirow{3}{*}{0.027} \\
\hline In & 80 & 0.02 & 0.04 & 0.00 & 0.01 & 0.03 & \\
\hline Total & 88 & 0.02 & 0.06 & 0.01 & 0.01 & 0.03 & \\
\hline Accepting SNAP benefits & & & & & & & \\
\hline Out & 42 & 0.26 & 0.445 & 0.069 & 0.12 & 0.40 & \multirow{3}{*}{0.007} \\
\hline In & 82 & 0.51 & 0.503 & 0.056 & 0.40 & 0.62 & \\
\hline Total & 124 & 0.43 & 0.497 & 0.045 & 0.34 & 0.52 & \\
\hline Matching programs for SNAP benefits & & & & & & & \\
\hline Out & 42 & 0.07 & 0.261 & 0.040 & -0.01 & 0.15 & \multirow{3}{*}{0.019} \\
\hline In & 82 & 0.24 & 0.432 & 0.048 & 0.15 & 0.34 & \\
\hline Total & 124 & 0.19 & 0.390 & 0.035 & 0.12 & 0.25 & \\
\hline Transportation services for consumers to access operation & & & & & & & \\
\hline Out & 42 & 0.02 & 0.154 & 0.024 & -0.02 & 0.07 & \multirow{3}{*}{0.050} \\
\hline In & 82 & 0.13 & 0.343 & 0.038 & 0.06 & 0.21 & \\
\hline Total & 124 & 0.10 & 0.297 & 0.027 & 0.04 & 0.15 & \\
\hline Food donation to local food pantries and banks & & & & & & & \\
\hline Out & 47 & 0.72 & 0.452 & 0.066 & 0.59 & 0.86 & \multirow{3}{*}{0.015} \\
\hline In & 103 & 0.88 & 0.322 & 0.032 & 0.82 & 0.95 & \\
\hline Total & 150 & 0.83 & 0.374 & 0.031 & 0.77 & 0.89 & \\
\hline
\end{tabular}

Source: Author's calculations.

Note: SNAP = Supplemental Nutrition Assistance Program. 
Table 4.8. Matrix of Adjusted Rand Index Values for Selected Models and Partitioning Solutions

\begin{tabular}{|c|c|c|c|c|c|c|c|c|c|c|c|c|c|}
\hline & & Model & 1 & 2 & 3 & 4 & 5 & 6 & 7 & 8 & 1 & 5 & 5 \\
\hline & & Method & Ward's & Complete & Average & Single & Ward's & Complete & Average & Single & Ward's & Ward's & Ward's \\
\hline Model & Method & Partition & 11 & 12 & 3 & 2 & 9 & 3 & 2 & 2 & 4 & 4 & 6 \\
\hline 1 & Ward's & 11 & 1.000 & & & & & & & & & & \\
\hline 2 & Complete & 12 & 0.479 & 1.000 & & & & & & & & & \\
\hline 3 & Average & 3 & 0.009 & 0.020 & 1.000 & & & & & & & & \\
\hline 4 & Single & 2 & 0.004 & 0.010 & 0.660 & 1.000 & & & & & & & \\
\hline 5 & Ward's & 9 & 0.248 & 0.249 & 0.010 & 0.005 & 1.000 & & & & & & \\
\hline 6 & Complete & 3 & 0.007 & 0.051 & 0.133 & 0.076 & 0.097 & 1.000 & & & & & \\
\hline 7 & Average & 2 & 0.008 & 0.023 & 0.377 & 0.483 & 0.017 & 0.223 & 1.000 & & & & \\
\hline 8 & Single & 2 & 0.005 & 0.016 & 0.484 & 0.656 & 0.010 & 0.151 & 0.787 & 1.000 & & & \\
\hline 1 & Ward's & 4 & 0.440 & 0.655 & 0.007 & 0.003 & 0.294 & 0.033 & 0.012 & 0.007 & 1.000 & & \\
\hline 5 & Ward's & 4 & 0.200 & 0.314 & 0.027 & 0.013 & 0.508 & 0.116 & 0.042 & 0.027 & 0.381 & 1.000 & \\
\hline 5 & Ward's & 6 & 0.245 & 0.333 & 0.011 & 0.005 & 0.786 & 0.142 & 0.019 & 0.012 & 0.349 & 0.689 & 1.000 \\
\hline
\end{tabular}

Source: Author's calculations.

Note: Values in bold indicate high degree of congruence in case assignments between clustering solutions. 
Table 4.9. Normalized Raw Stress Values of the Final Variable Sets for 2 Through 4 Dimensions

\begin{tabular}{|c|c|c|}
\hline \multirow{2}{*}{$\begin{array}{c}\text { Number of } \\
\text { Dimensions }\end{array}$} & Eormalized Raw Stress for Variable Set \\
\cline { 2 - 3 } & 0.063 & F \\
\hline 2 & 0.030 & 0.075 \\
\hline 3 & 0.020 & 0.038 \\
\hline 4 & & 0.026 \\
\hline
\end{tabular}

Source: Author's calculations. 
Figure 4.5. Representation of Cases in Three-Dimensional Space: Model 1, $k=11$

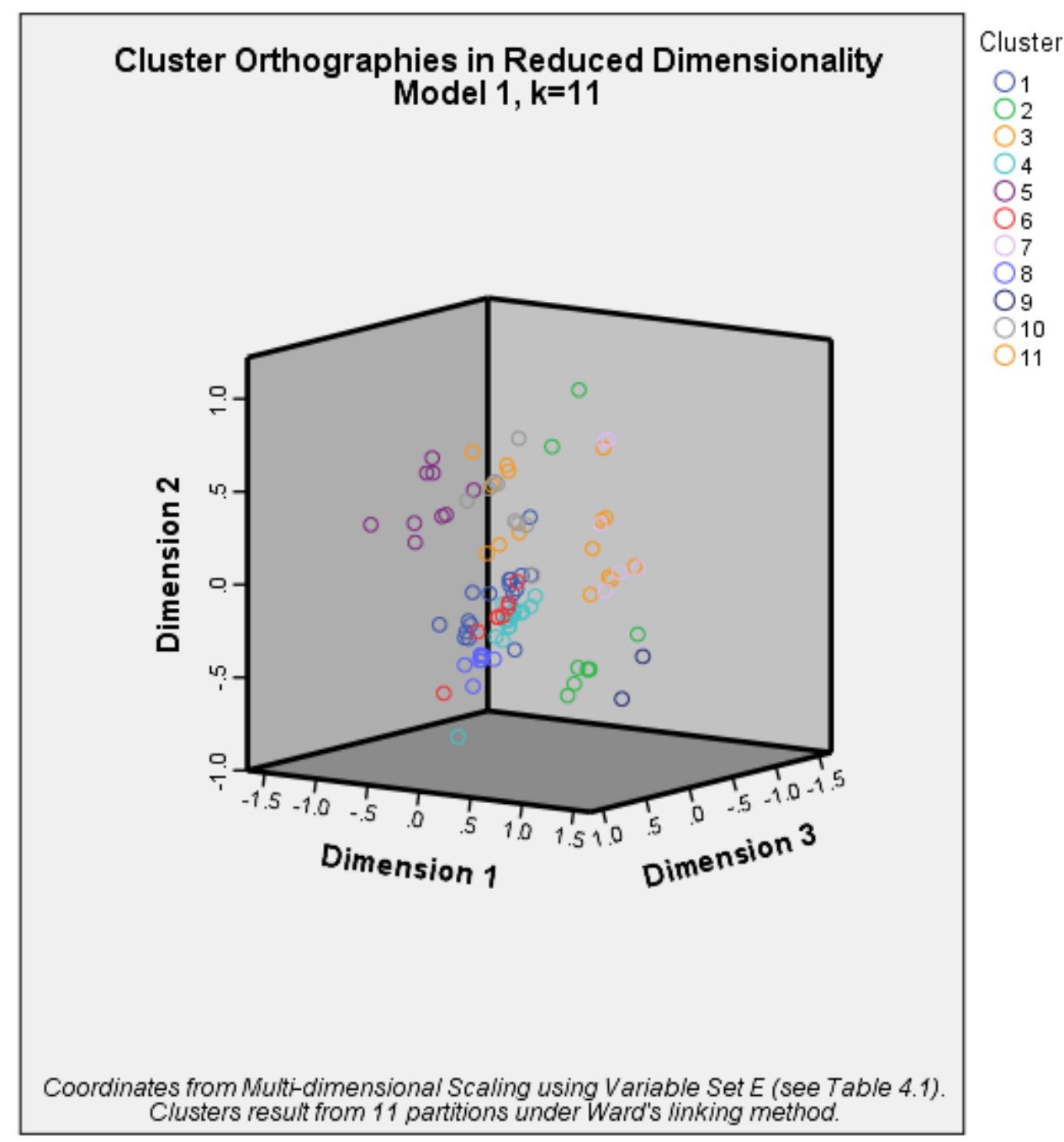

Source: Author. 
Figure 4.6. Representation of Cases in Three-Dimensional Space: Model 2, $k=12$

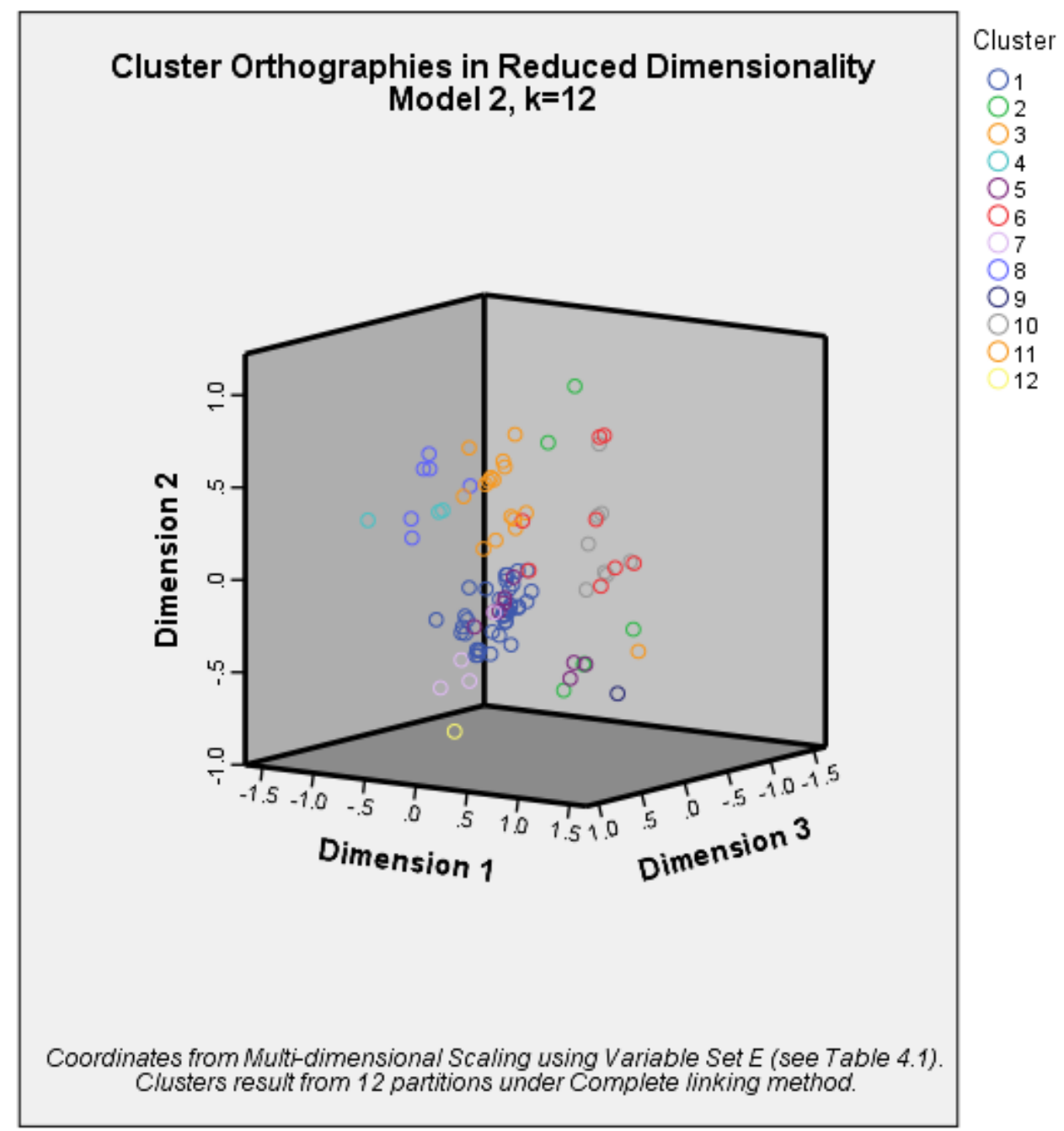

Source: Author. 
Figure 4.7. Representation of Cases in Three-Dimensional Space: Model 5, $k=9$

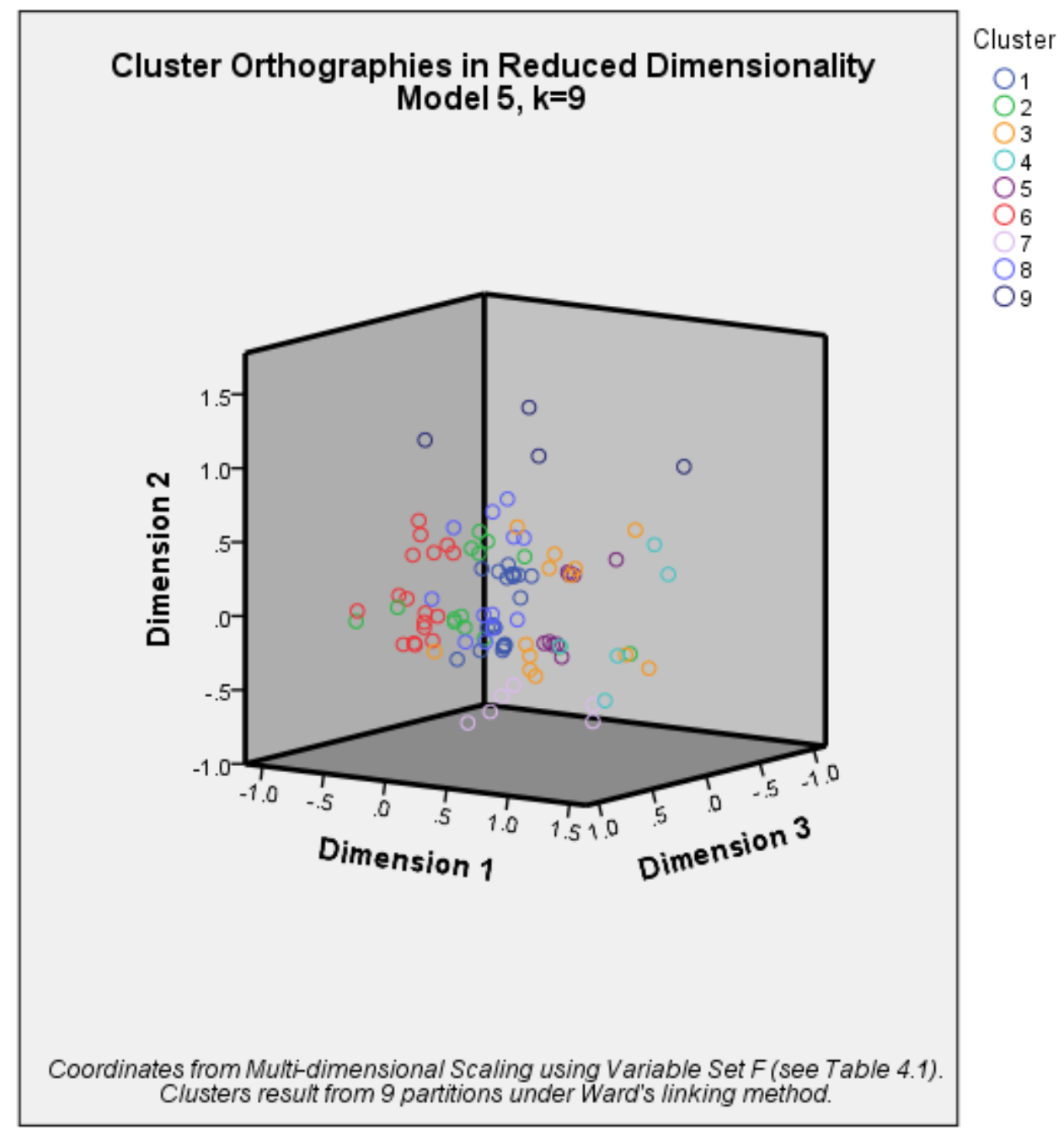

Source: Author. 
Figure 4.8. Representation of Cases in Three-Dimensional Space: Model 6, $k=3$

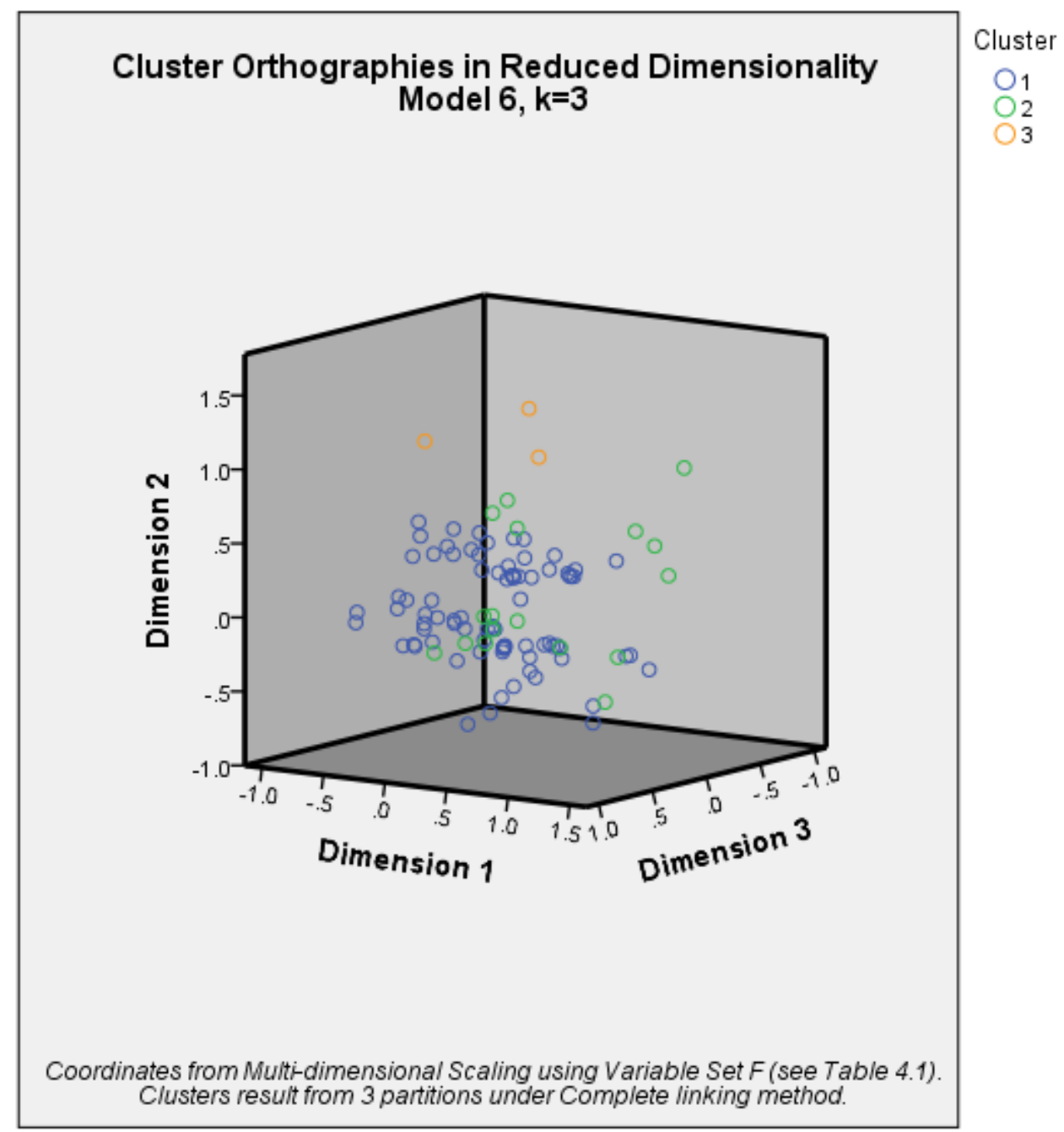

Source: Author. 
Table 4.10. Node Heights and Differences for Model 5

\begin{tabular}{|c|c|c|c|}
\hline $\begin{array}{c}\text { Number of } \\
\text { Partitions }(\boldsymbol{k}=)\end{array}$ & Height & Difference & Rank of Difference \\
\hline 2 & 39.95 & 0.00 & 0 \\
\hline 3 & 35.85 & 4.10 & 4 \\
\hline 4 & 26.30 & 9.55 & 1 \\
\hline 5 & 21.98 & 4.32 & 3 \\
\hline 6 & 21.62 & 0.36 & 9 \\
\hline 7 & 14.54 & 7.08 & 2 \\
\hline 8 & 13.90 & 0.64 & 10 \\
\hline 9 & 13.74 & 0.16 & 7 \\
\hline 10 & 12.44 & 1.30 & 6 \\
\hline 11 & 10.58 & 1.86 & 5 \\
\hline 12 & 8.14 & 2.44 & \\
\hline
\end{tabular}

Source: Author. 
Table 4.11. Summary of Statistical Significance of Multigroup Analysis of Variance for Selected Partitioning Solutions of Model 5: Morphological Variables

\begin{tabular}{|c|c|c|c|c|c|c|}
\hline \multirow[b]{2}{*}{ Variable } & \multicolumn{6}{|c|}{ No. of Partitions $(k=)$} \\
\hline & 4 & 5 & 6 & 7 & 8 & 9 \\
\hline Natural log of total no. of years in business & 0.415 & 0.117 & 0.111 & 0.132 & 0.133 & 0.054 \\
\hline Profit motivated legal status & 0.169 & 0.101 & 0.093 & 0.146 & 0.208 & 0.210 \\
\hline Cooperative form legal status & 0.000 & 0.000 & 0.000 & 0.000 & 0.000 & 0.000 \\
\hline Acts as broker & 0.001 & 0.001 & 0.003 & 0.000 & 0.000 & 0.000 \\
\hline Percentage of revenue attributed to sources other than sales & 0.001 & 0.002 & 0.004 & 0.001 & 0.002 & 0.002 \\
\hline Total warehouse space in square feet & 0.143 & 0.215 & 0.038 & 0.001 & 0.002 & 0.000 \\
\hline Number of delivery vehicles on hand & 0.098 & 0.168 & 0.017 & 0.002 & 0.005 & 0.008 \\
\hline Offer transport services to producers & 0.000 & 0.000 & 0.000 & 0.000 & 0.000 & 0.000 \\
\hline Additional packaging, involvement level & 0.013 & 0.003 & 0.008 & 0.010 & 0.015 & $\mathbf{0 . 0 2 2}$ \\
\hline Processing facilities & 0.000 & 0.000 & 0.000 & 0.000 & 0.000 & 0.000 \\
\hline Online ordering system & 0.327 & 0.488 & 0.577 & 0.280 & 0.386 & 0.211 \\
\hline Rental space for other businesses & 0.000 & 0.000 & 0.000 & 0.000 & 0.000 & 0.000 \\
\hline Retail space for the hub & 0.000 & 0.000 & 0.000 & 0.000 & 0.000 & 0.000 \\
\hline Licensed shared use kitchen & 0.000 & 0.000 & 0.000 & 0.000 & 0.000 & 0.000 \\
\hline
\end{tabular}

Source: Author's calculations.

Note: Values in bold indicate statistical significance at or beyond the 10 percent level. 
Figure 4.9. Error Bar Chart: Variables Representing Organization Morphology

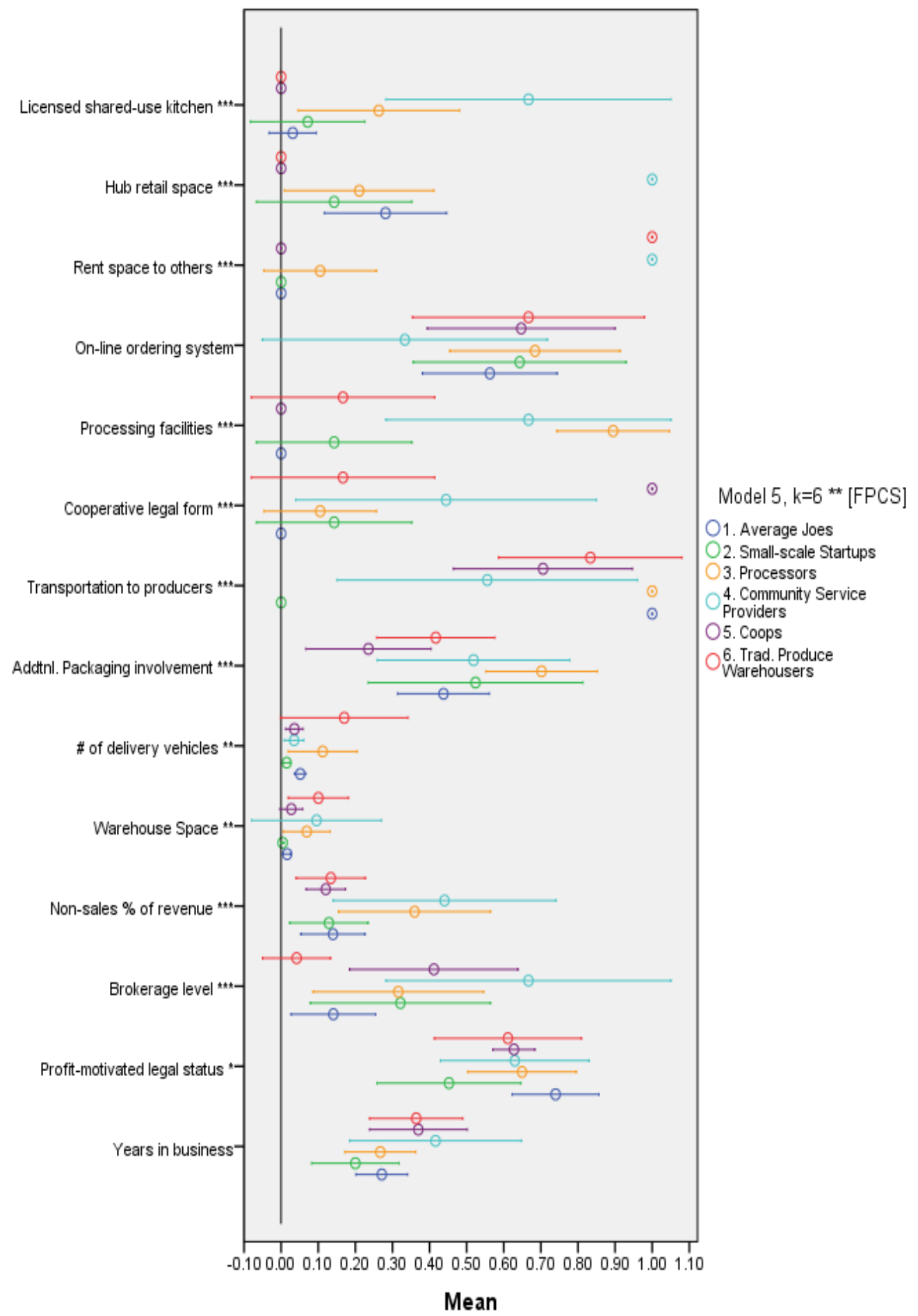

Error Bars: $95 \% \mathrm{Cl}$

Source: Author.

Note: Asterisks indicate statistical significance for group analysis of variance $F$-statistic: $* 10 \%, * * 5 \%$, and $* * * 1 \%$. FPCS = final postulated cluster solution. 
Table 4.12. Summary of Species Populations and Their Niche, as Represented by Strategic Orientations

\begin{tabular}{|c|c|c|c|c|c|c|c|}
\hline & & & & & & ck Summary & \\
\hline Species & $N$ & $\begin{array}{l}\text { Percentage } \\
\text { of Total }\end{array}$ & $\begin{array}{l}\text { Percentage } \\
\text { of Sample }\end{array}$ & Strategic Orientation & Product Reliance & $\begin{array}{l}\text { Channel } \\
\text { Reliance }\end{array}$ & $\begin{array}{c}\text { Community } \\
\text { Services }\end{array}$ \\
\hline 1. Average joes & 32 & 21.2 & 31.1 & $\begin{array}{l}\text { Highly dependent on fresh produce } \\
\text { (65.3\%) supplemented by meat and } \\
\text { poultry }(14.36 \%) \\
\text { Most sales to DTR channels ( } 52.98 \%) \\
\text { with some DTC ( } 28.98 \%) \text { and DTI } \\
(13.35 \%) \\
\text { Offer roughly the sample average no. of } \\
\text { community services }(4.97)\end{array}$ & $\begin{array}{l}\text { Fresh produce }+ \\
\text { meat and poultry }\end{array}$ & $\begin{array}{l}\text { DTR + DTC } \\
\& \text { DTI }\end{array}$ & Average \\
\hline 2. Small-scale startups & 14 & 9.3 & 13.6 & $\begin{array}{l}\text { Predominantly fresh produce }(53.86 \%) \\
\text { with a higher share in meat and poultry } \\
(24.8 \%) \text { to supplement } \\
\text { Most sales go to DTC channels } \\
\text { (64.14\%) with some DTR (20.05\%) } \\
\text { Offer few community services (3.71), } \\
\text { especially those focused on community } \\
\text { food systems issues }\end{array}$ & $\begin{array}{l}\text { Fresh produce }+ \\
\text { meat and poultry }\end{array}$ & DTC + DTR & Fewer \\
\hline 3. Processors & 19 & 12.6 & 18.4 & $\begin{array}{l}\text { - Greatest reliance on processed products: } \\
\text { processed produce }(12.84 \%) \text { and other } \\
\text { value added }(9.89 \%) \text {, largest of any } \\
\text { group } \\
\text { - Fresh produce still a plurality at } 46.01 \% \\
\text { - Sales channels are diversified, with } \\
\text { roughly half }(49.04 \%) \text { to DTC and the } \\
\text { remaining relatively evenly split among } \\
\text { DTR, DTM, and DTI } \\
\text { Offer slightly more community services } \\
\text { than average at } 5.95\end{array}$ & $\begin{array}{l}\text { Fresh produce }+ \\
\text { processed products }\end{array}$ & DTC & Slightly more \\
\hline
\end{tabular}




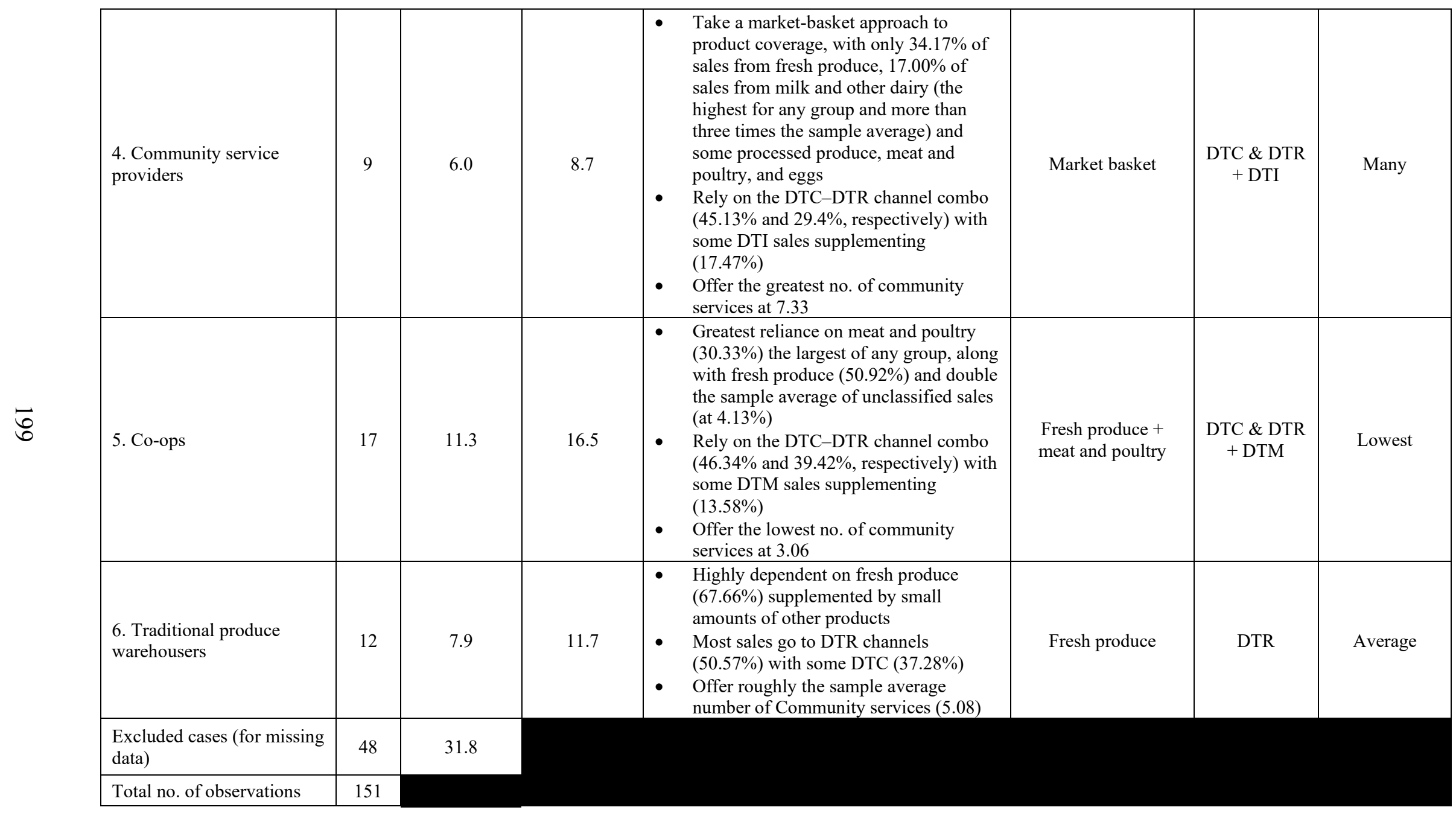

Source: Author.

Note: DTC = direct to consumers; DTI = direct to institutions; DTM = direct to intermediaries; DTR = direct to retailers and restaurants. 
Figure 4.10. Dendrogram of Clustering Solutions Under Model 5 With the Six Partitions of the Final Postulated Solution and Species Names Indicated $(k=6)$

\section{Dendrogram for Model 5, $k=6$}

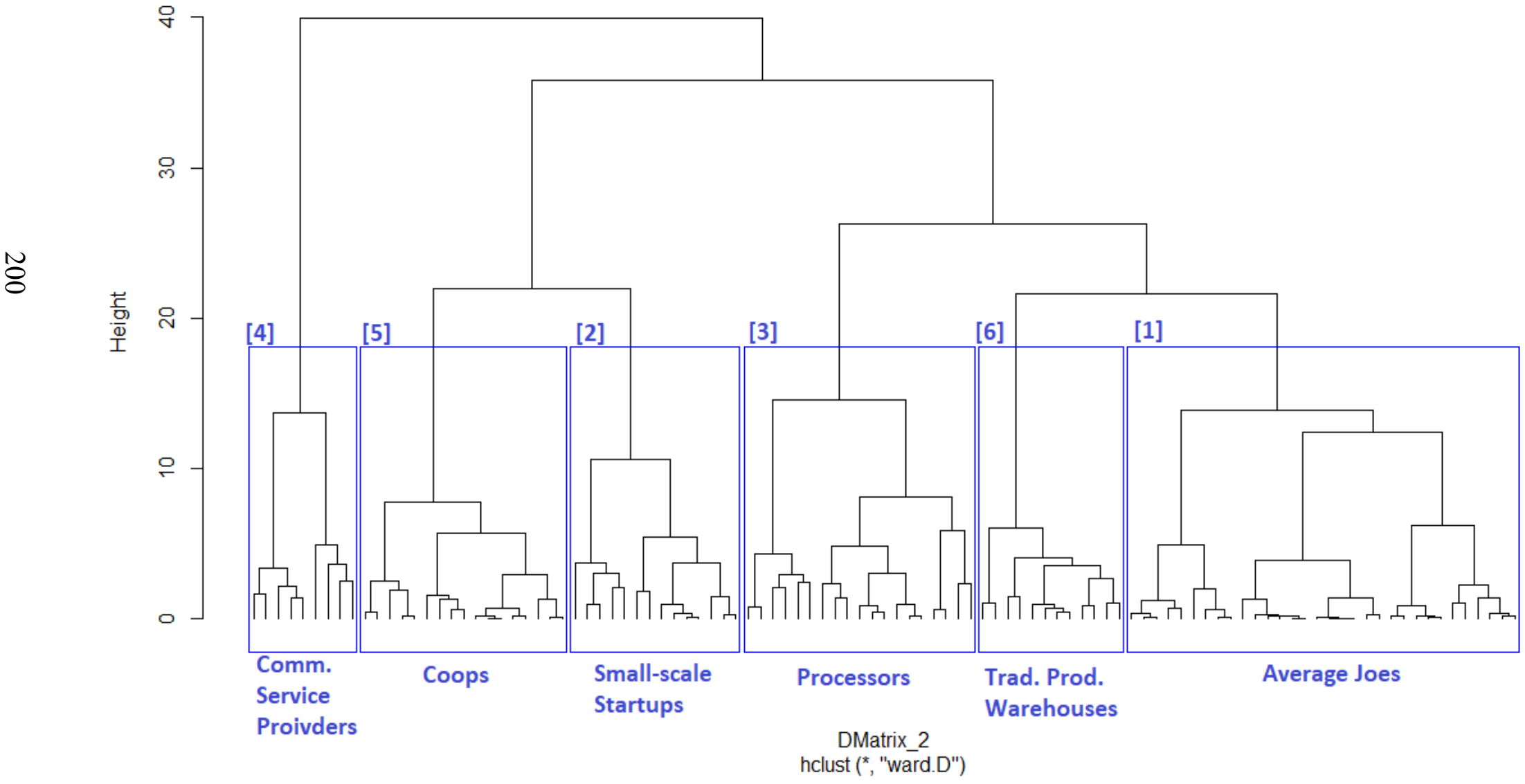


Figure 4.11. Representation of Cases in Three-Dimensional Space: Model 5, $k=6$

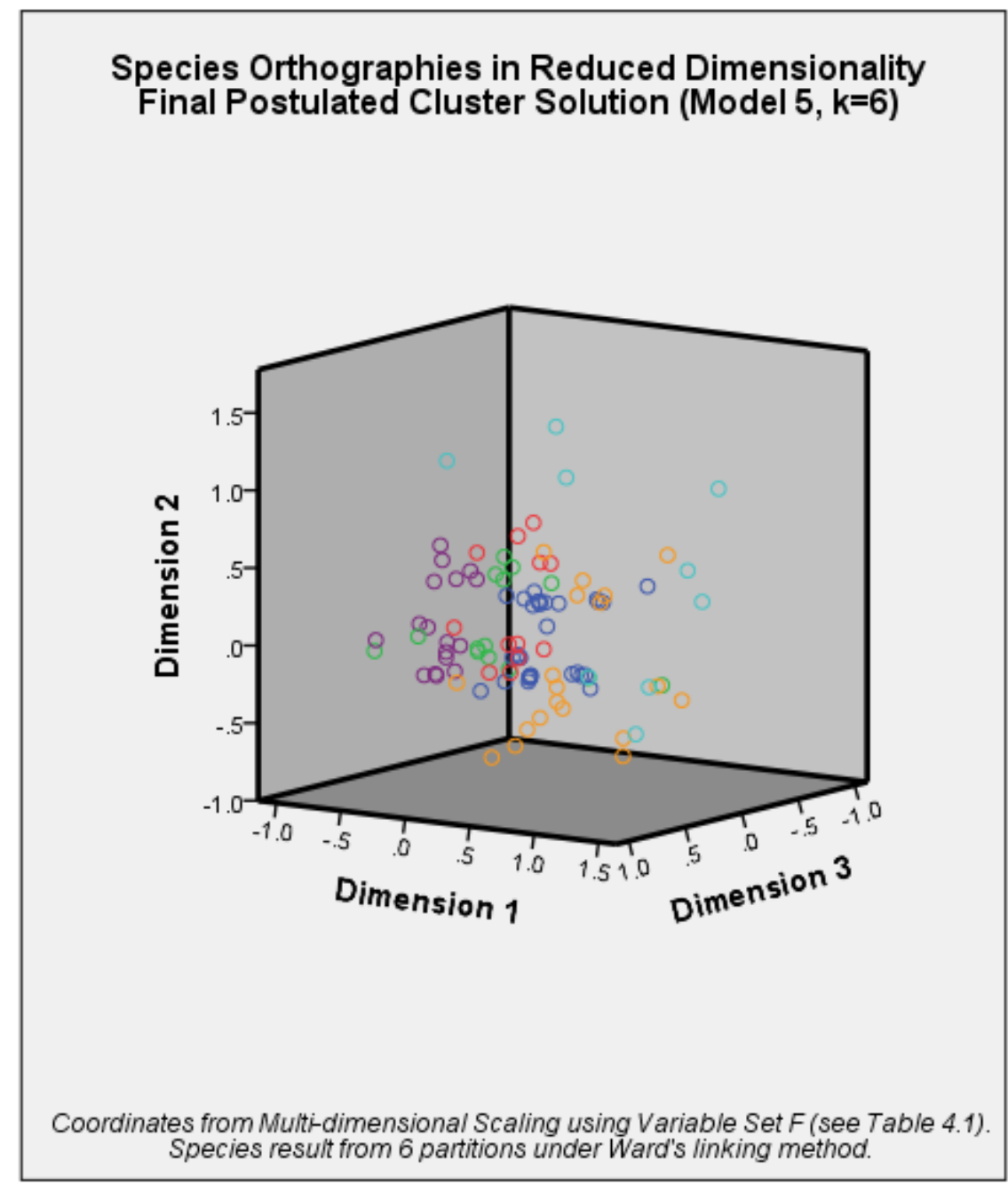

Species

O1. Average Joes

2. Small-scale Startups

3. Processors

4. Community Service

Providers

5. Coops

6. Traditional Produce

Warehousers

Source: Author. 
Figure 4.12. Error Bar Chart: Variables Representing Channel and Product Reliance

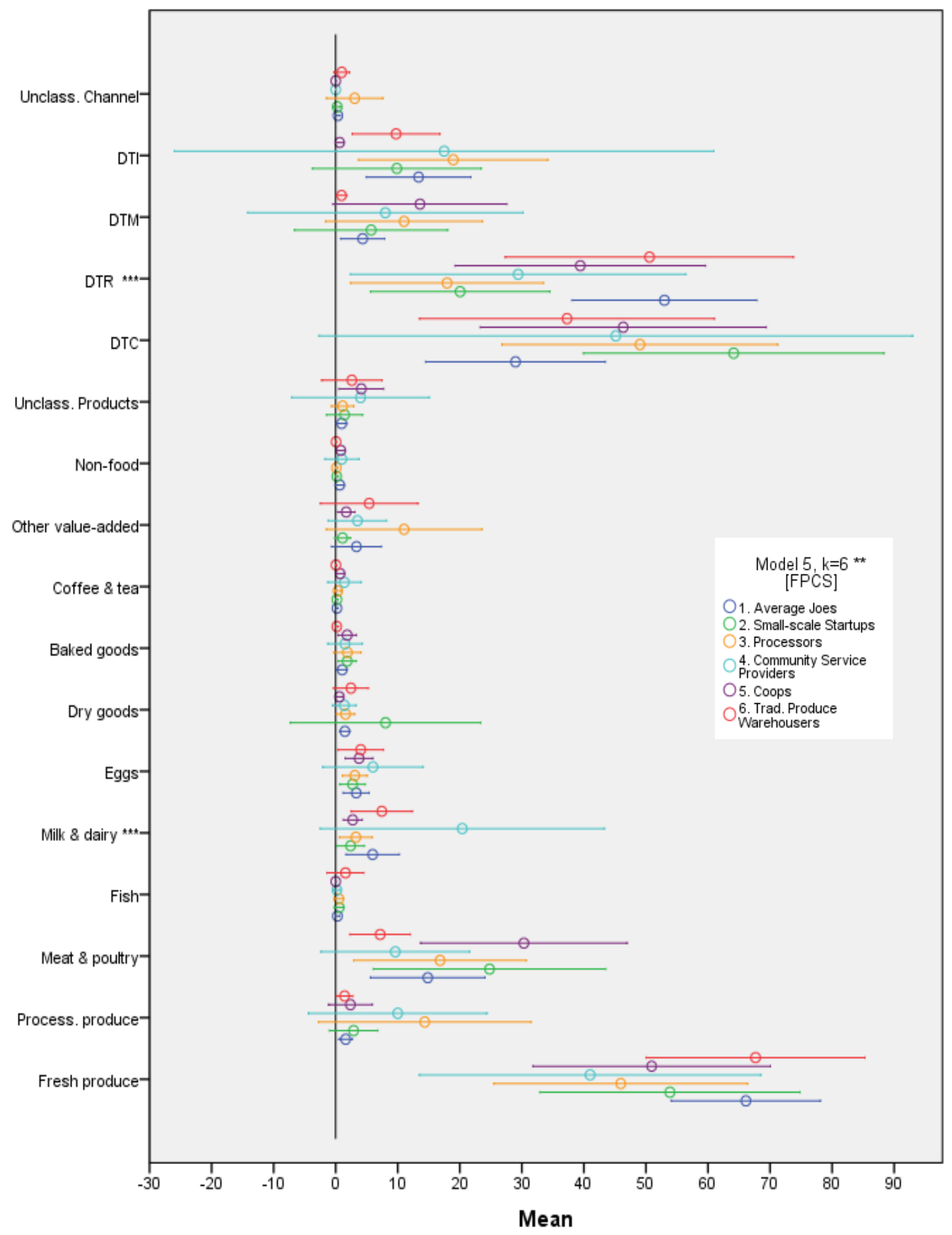

Error Bars: $95 \% \mathrm{Cl}$

Source: Author.

Note: Asterisks indicate statistical significance for group analysis of variance $F$-statistic: $* 10 \%$, $* * 5 \%$, and $* * * 1 \%$. FPCS = final postulated cluster solution. 
Figure 4.13. Error Bar Chart: Variables Representing Community Services

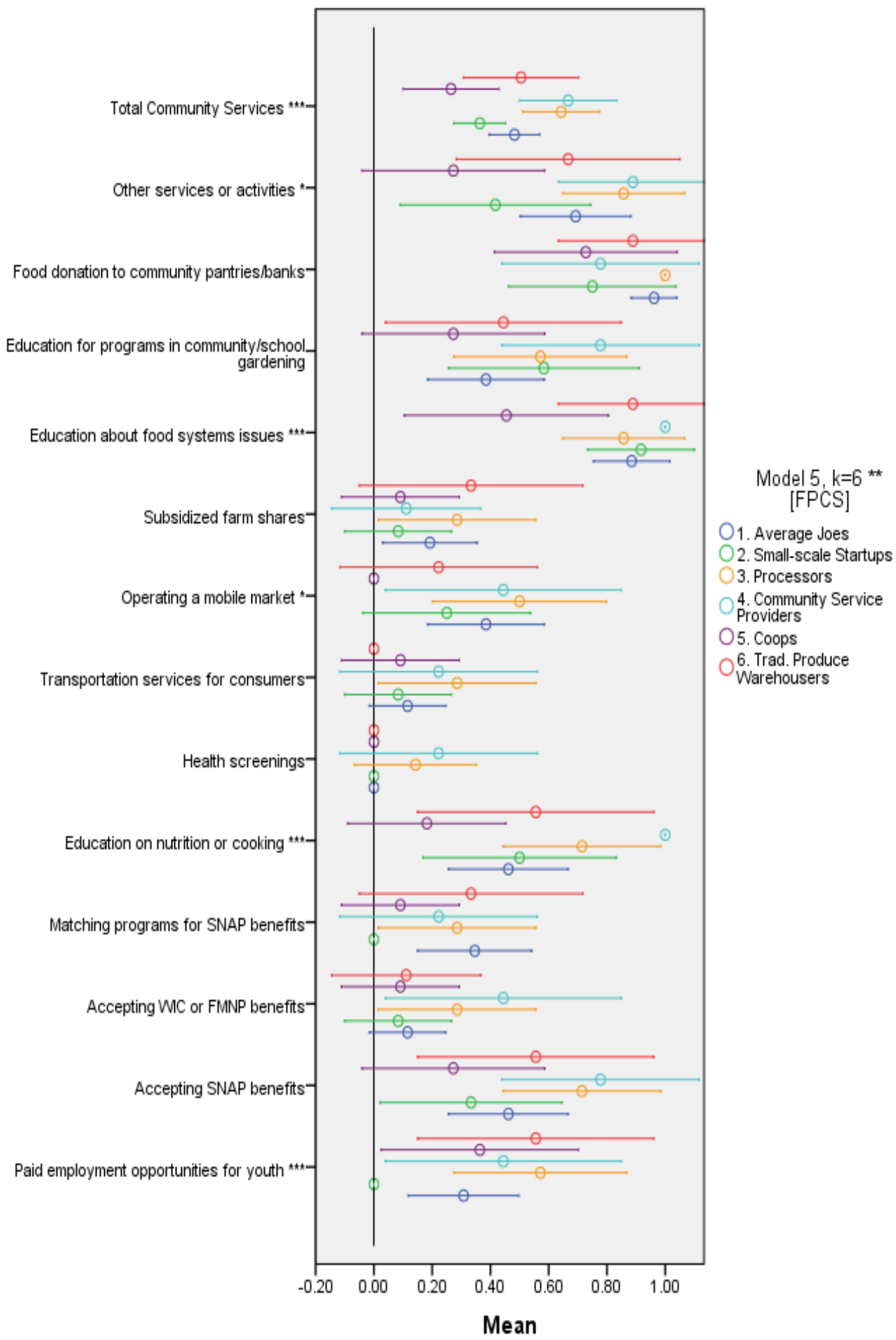

Error Bars: $95 \% \mathrm{Cl}$

Source: Author.

Note: Asterisks indicate statistical significance for group analysis of variance $F$-statistic: $* 10 \%, * * 5 \%$, and $* * * 1 \%$. FPCS $=$ final postulated cluster solution. 
Table 4.13 Summary of Statistical Significance of Multigroup Analysis of Variance for Selected Partitioning Solutions of Model 5: Niche Variables for Channel and Product Reliance

\begin{tabular}{|c|c|c|c|c|c|c|}
\hline \multirow[b]{2}{*}{ Variable } & \multicolumn{6}{|c|}{ No. of Partitions $(k=)$} \\
\hline & 4 & 5 & 6 & 7 & 8 & 9 \\
\hline Sales direct to consumers & 0.115 & 0.118 & 0.175 & 0.255 & 0.357 & 0.464 \\
\hline Sales direct to retailers and restaurants & 0.004 & 0.004 & 0.009 & $\mathbf{0 . 0 1 7}$ & 0.028 & 0.041 \\
\hline Sales direct to intermediaries & 0.378 & 0.351 & 0.456 & 0.588 & 0.705 & 0.735 \\
\hline Sales direct to institutions & 0.153 & 0.156 & 0.232 & 0.197 & 0.232 & $\mathbf{0 . 1 0 0}$ \\
\hline Unclassified channels & 0.074 & 0.141 & 0.214 & 0.312 & 0.414 & 0.526 \\
\hline Fresh produce and herbs & 0.048 & 0.094 & 0.161 & 0.147 & 0.218 & 0.303 \\
\hline Processed produce & 0.039 & 0.079 & 0.140 & $\mathbf{0 . 0 2 7}$ & 0.046 & $\mathbf{0 . 0 7 1}$ \\
\hline Meat and poultry & 0.054 & 0.093 & 0.125 & 0.165 & 0.223 & 0.295 \\
\hline Fish & 0.728 & 0.745 & 0.418 & 0.461 & 0.575 & 0.679 \\
\hline Milk and other dairy products & 0.001 & 0.003 & $\mathbf{0 . 0 0 7}$ & 0.014 & $\mathbf{0 . 0 1 7}$ & $\mathbf{0 . 0 0 0}$ \\
\hline Eggs & 0.778 & 0.837 & 0.910 & 0.942 & 0.947 & 0.939 \\
\hline Grains, beans, and flours & 0.778 & 0.274 & 0.395 & 0.525 & 0.645 & 0.747 \\
\hline Baked goods and bread & 0.591 & 0.754 & 0.660 & 0.679 & 0.772 & 0.841 \\
\hline Coffee and tea & 0.232 & 0.170 & 0.192 & 0.113 & 0.124 & 0.117 \\
\hline Other processed or value-added food products & 0.143 & 0.247 & 0.341 & 0.255 & 0.325 & 0.428 \\
\hline Nonfood items & 0.579 & 0.457 & 0.310 & 0.404 & $\mathbf{0 . 0 3 7}$ & $\mathbf{0 . 0 2 7}$ \\
\hline Unclassified products & 0.450 & 0.314 & 0.337 & 0.298 & 0.362 & 0.248 \\
\hline
\end{tabular}

Source: Author's calculations.

Note: Values in bold indicate statistical significance at or beyond the 10 percent level. 
Table 4.14 Summary of Statistical Significance of Multigroup Analysis of Variance for Selected Partitioning solutions of Model 5: Niche Variables for Community Service Offerings

\begin{tabular}{|c|c|c|c|c|c|c|}
\hline \multirow[b]{2}{*}{ Variable } & \multicolumn{6}{|c|}{ No. of Partitions $(k=)$} \\
\hline & 4 & 5 & 6 & 7 & 8 & 9 \\
\hline Paid employment opportunities for youth & 0.008 & 0.008 & 0.003 & 0.000 & $\mathbf{0 . 0 0 0}$ & $\mathbf{0 . 0 0 0}$ \\
\hline Accepting SNAP benefits & 0.045 & 0.091 & 0.145 & 0.199 & 0.251 & 0.343 \\
\hline Accepting WIC or FMNP benefits & 0.047 & 0.095 & 0.164 & 0.200 & 0.209 & 0.283 \\
\hline Matching programs for SNAP benefits & 0.150 & 0.184 & 0.290 & 0.350 & 0.390 & 0.315 \\
\hline Nutrition or cooking education & 0.001 & 0.001 & $\mathbf{0 . 0 0 3}$ & 0.002 & $\mathbf{0 . 0 0 3}$ & 0.006 \\
\hline Health screenings & 0.065 & 0.126 & 0.179 & 0.011 & 0.020 & 0.002 \\
\hline Transportation services for consumers to access your operation & 0.209 & 0.341 & 0.386 & 0.412 & 0.532 & 0.329 \\
\hline Operating a mobile market & 0.071 & 0.066 & $\mathbf{0 . 0 8 7}$ & 0.127 & 0.182 & $\mathbf{0 . 0 2 7}$ \\
\hline Subsidized farm shares & 0.557 & 0.677 & 0.680 & 0.711 & 0.803 & 0.827 \\
\hline Education about community and food systems issues & 0.130 & 0.003 & 0.007 & 0.012 & 0.011 & $\mathbf{0 . 0 2 0}$ \\
\hline Education for programs in community or school gardening & 0.219 & 0.221 & 0.335 & 0.319 & 0.430 & 0.323 \\
\hline Food donation to local food pantries and banks & $\mathbf{0 . 0 5 7}$ & 0.078 & 0.126 & 0.186 & 0.262 & 0.068 \\
\hline Other community services or activities & 0.024 & 0.051 & 0.094 & 0.084 & 0.111 & 0.149 \\
\hline Total no. of community services offered & 0.000 & 0.000 & 0.000 & 0.000 & 0.000 & $\mathbf{0 . 0 0 0}$ \\
\hline
\end{tabular}

Source: Author's calculations.

Note: Values in bold indicate statistical significance at or beyond the 10 percent level. FMNP $=$ Farmers Market Nutrition Program; SNAP $=$ Supplemental Nutrition Assistance Program; WIC = Women Infants and Children (program). 
Table 4.15 Mean Differences in Species Morphological Variables: Each Species Compared With All Other Observations

\begin{tabular}{|c|c|c|c|c|c|c|}
\hline Variable & $\begin{array}{c}\text { Average } \\
\text { Joes }\end{array}$ & $\begin{array}{l}\text { Small-Scale } \\
\text { Startups }\end{array}$ & Processors & $\begin{array}{c}\text { Community } \\
\text { Service } \\
\text { Providers }\end{array}$ & Co-ops & $\begin{array}{c}\text { Traditional Produce } \\
\text { Warehousers }\end{array}$ \\
\hline Profit motivated & -0.44 & 0.57 & -0.08 & -0.02 & -0.01 & 0.04 \\
\hline Cooperative form & 0.28 & 0.08 & 0.13 & -0.24 & -0.88 & 0.06 \\
\hline Firm age $(\ln )$ & 0.12 & 0.49 & 0.13 & -0.67 & -0.45 & -0.40 \\
\hline Acts as broker & 0.28 & -0.16 & -0.15 & -0.89 & -0.36 & 0.45 \\
\hline Nonsales percentage of revenue & 0.08 & 0.08 & -0.19 & -0.26 & 0.09 & 0.07 \\
\hline Total warehouse space & 6411.11 & 8322.20 & -7100.59 & -12484.25 & 2930.49 & -13952.26 \\
\hline No. of delivery vehicles & 0.68 & 2.53 & -2.73 & 1.42 & 1.47 & -5.64 \\
\hline Offer transport services to producers & -0.39 & 0.78 & -0.35 & 0.15 & -0.01 & -0.15 \\
\hline Additional packaging involvement & 0.10 & -0.21 & -0.83 & -0.18 & 0.78 & 0.15 \\
\hline Processing facilities & 0.34 & 0.13 & -0.73 & -0.43 & 0.30 & 0.10 \\
\hline Online ordering system & 0.04 & -0.06 & -0.10 & 0.28 & -0.06 & -0.08 \\
\hline Rental space for other businesses & 0.24 & 0.21 & 0.09 & -0.87 & 0.21 & -0.89 \\
\hline Retail space for the hub & -0.08 & 0.09 & 0.01 & -0.83 & 0.25 & 0.24 \\
\hline Licensed shared use kitchen & 0.15 & 0.08 & -0.14 & -0.56 & 0.16 & 0.16 \\
\hline
\end{tabular}

Source: Author's calculations.

Note: Values in bold indicate statistical significance at or beyond the 10 percent level. 
Table 4.16 Mean Differences in Species Niche Variables: Each Species Compared With All Other Observations

\begin{tabular}{|c|c|c|c|c|c|c|}
\hline Variable & $\begin{array}{c}\text { Average } \\
\text { Joes }\end{array}$ & $\begin{array}{c}\text { Small- } \\
\text { Scale } \\
\text { Startups }\end{array}$ & Processors & CSPs & Coops & TPWs \\
\hline Fresh produce and herbs & -11.25 & 3.74 & 13.39 & 24.26 & 7.32 & -11.77 \\
\hline Processed produce & 3.43 & 1.51 & -10.38 & -4.36 & 2.16 & 3.11 \\
\hline Meat and poultry & 2.84 & -9.56 & 1.32 & 8.90 & -16.36 & 10.37 \\
\hline Fish & 0.02 & -0.15 & -0.01 & 0.29 & 0.51 & -1.27 \\
\hline Milk and other dairy products & -1.65 & 3.14 & 2.68 & -12.52 & 2.82 & -2.56 \\
\hline Eggs & -0.02 & 0.91 & 0.86 & -1.56 & -0.27 & -0.57 \\
\hline Grains, beans, and flours & 1.71 & -5.97 & 1.65 & 1.72 & 2.58 & 0.39 \\
\hline Baked goods and bread & 0.08 & -0.57 & -0.40 & 0.11 & -0.57 & 1.32 \\
\hline Coffee and tea & 0.04 & 0.26 & 0.14 & -0.79 & -0.36 & 0.42 \\
\hline Other processed or value added & 1.83 & 3.90 & -6.46 & 1.69 & 3.32 & -0.98 \\
\hline Nonfood items & -0.45 & 0.31 & 0.41 & -0.41 & -0.44 & 0.44 \\
\hline All unclassified sales & 1.17 & 0.36 & 0.89 & -1.68 & -2.81 & -0.97 \\
\hline Direct to consumers & 20.50 & -23.26 & -6.08 & -1.27 & -2.88 & 7.49 \\
\hline Direct to retailers and restaurants & -21.14 & 20.16 & 23.33 & 8.57 & -2.20 & -14.64 \\
\hline Direct to intermediaries & 3.47 & 1.33 & -4.92 & -1.18 & -7.98 & 6.68 \\
\hline Direct to institutions & -3.45 & 1.14 & -9.63 & -6.96 & 12.13 & 1.25 \\
\hline Unclassified & 0.53 & 0.56 & -2.76 & 0.77 & 0.87 & -0.27 \\
\hline Total community services offered & -0.26 & 1.16 & -1.35 & -2.73 & 1.93 & -0.34 \\
\hline Paid employment opportunities for youth & 0.04 & 0.35 & -0.30 & -0.14 & 0.09 & -0.29 \\
\hline Accepting SNAP benefits & -0.04 & 0.10 & -0.32 & -0.38 & 0.10 & -0.14 \\
\hline Accepting WIC or FMNP benefits & 0.08 & 0.11 & -0.12 & -0.29 & 0.10 & 0.07 \\
\hline Matching programs for SNAP benefits & -0.20 & 0.21 & -0.11 & -0.04 & 0.02 & -0.16 \\
\hline Nutrition or cooking education & 0.09 & 0.09 & -0.08 & -0.52 & 0.37 & 0.10 \\
\hline Health screenings & 0.04 & 0.07 & -0.05 & -0.17 & 0.07 & -0.02 \\
\hline $\begin{array}{l}\text { Transportation services for consumers to } \\
\text { access your operation }\end{array}$ & -0.02 & 0.01 & -0.21 & -0.14 & 0.01 & 0.10 \\
\hline Operating a mobile market & -0.15 & 0.02 & -0.26 & -0.19 & 0.29 & 0.05 \\
\hline Subsidized farm shares & -0.01 & 0.11 & -0.11 & 0.08 & 0.02 & -0.16 \\
\hline $\begin{array}{l}\text { Education about community and food } \\
\text { systems issues }\end{array}$ & -0.07 & -0.15 & 0.06 & -0.22 & 0.36 & -0.05 \\
\hline $\begin{array}{l}\text { Education for programs in community or } \\
\text { school gardening }\end{array}$ & 0.05 & -0.02 & 0.01 & -0.32 & 0.21 & 0.07 \\
\hline $\begin{array}{l}\text { Food donation to local food pantries and } \\
\text { banks }\end{array}$ & -0.17 & 0.13 & -0.13 & 0.06 & 0.01 & -0.09 \\
\hline Other community services or activities & 0.01 & 0.28 & -0.12 & -0.22 & 0.24 & 0.02 \\
\hline
\end{tabular}

Source: Author's calculations.

Note: Values in bold indicate statistical significance at or beyond the 10 percent level. FMNP $=$ Farmers Market Nutrition Program; SNAP = Supplemental Nutrition Assistance Program; and WIC = Women Infants and Children (program). 
Table 4.17 Mean Differences in Species Product and Channel Reliance Variables: Species-to-Species Comparisons

\begin{tabular}{|c|c|c|c|c|c|c|c|c|c|c|c|c|c|c|c|}
\hline Variable & 1 vs. 2 & 1 vs. 3 & 1 vs. 4 & 1 vs. 5 & 1 vs. 6 & 2 vs. 3 & 2 vs. 4 & 2 vs. 5 & 2 vs. 6 & 3 vs. 4 & 3 vs. 5 & 3 vs. 6 & 4 vs. 5 & 4 vs. 6 & 5 vs. 6 \\
\hline $\begin{array}{l}\text { Fresh produce } \\
\text { and herbs }\end{array}$ & 11.44 & 19.30 & 31.14 & 14.39 & -2.36 & 7.86 & 19.70 & 2.94 & 2.94 & 11.84 & -4.92 & -21.66 & -16.76 & -33.50 & -16.74 \\
\hline $\begin{array}{l}\text { Processed } \\
\text { produce }\end{array}$ & -1.16 & -11.12 & -6.61 & -0.65 & 0.29 & -9.96 & -5.45 & 0.51 & 0.51 & 4.51 & 10.47 & 11.41 & 5.96 & 6.90 & 0.95 \\
\hline $\begin{array}{l}\text { Meat and } \\
\text { poultry }\end{array}$ & -10.44 & -0.96 & 6.36 & -15.96 & 7.20 & 9.48 & 16.80 & -5.53 & -5.53 & 7.32 & -15.00 & 8.16 & -22.33 & 0.84 & 23.17 \\
\hline Fish & -0.15 & -0.03 & 0.25 & 0.42 & -1.15 & 0.12 & 0.41 & 0.57 & 0.57 & 0.28 & 0.45 & -1.13 & 0.17 & -1.41 & -1.57 \\
\hline $\begin{array}{l}\text { Milk and other } \\
\text { dairy products }\end{array}$ & 3.95 & 3.43 & -10.66 & 3.60 & -1.09 & -0.51 & -14.61 & -0.35 & -0.35 & -14.09 & 0.16 & -4.52 & 14.25 & 9.57 & -4.68 \\
\hline Eggs & 0.81 & 0.73 & -1.46 & -0.22 & -0.50 & -0.08 & -2.28 & -1.03 & -1.03 & -2.20 & -0.95 & -1.23 & 1.25 & 0.97 & -0.28 \\
\hline $\begin{array}{l}\text { Grains, beans, } \\
\text { and flours }\end{array}$ & -6.47 & 0.13 & 0.38 & 0.95 & -0.90 & 6.60 & 6.86 & 7.42 & 7.42 & 0.25 & 0.82 & -1.02 & 0.57 & -1.28 & -1.85 \\
\hline $\begin{array}{l}\text { Baked goods } \\
\text { and bread }\end{array}$ & -0.56 & -0.39 & 0.05 & -0.54 & 1.12 & 0.17 & 0.60 & 0.01 & 0.01 & 0.43 & -0.15 & 1.51 & -0.59 & 1.08 & 1.67 \\
\hline Coffee and tea & 0.19 & 0.08 & -0.78 & -0.34 & 0.34 & -0.11 & -0.97 & -0.53 & -0.53 & -0.86 & -0.42 & 0.26 & 0.44 & 1.13 & 0.68 \\
\hline $\begin{array}{l}\text { Other processed } \\
\text { or value added }\end{array}$ & 2.09 & -6.70 & 0.27 & 1.49 & -2.21 & -8.79 & -1.82 & -0.60 & -0.60 & 6.97 & 8.20 & 4.50 & 1.22 & -2.48 & -3.70 \\
\hline Nonfood items & 0.60 & 0.66 & -0.06 & -0.05 & 0.72 & 0.07 & -0.66 & -0.65 & -0.65 & -0.73 & -0.72 & 0.05 & 0.01 & 0.78 & 0.77 \\
\hline $\begin{array}{l}\text { Unclassified } \\
\text { products }\end{array}$ & -0.53 & -0.10 & -2.44 & -3.23 & -1.71 & 0.43 & -1.91 & -2.70 & -2.70 & -2.33 & -3.13 & -1.60 & -0.79 & 0.73 & 1.52 \\
\hline $\begin{array}{l}\text { Direct to } \\
\text { consumers }\end{array}$ & -35.16 & -20.06 & -16.15 & -17.36 & -8.30 & 15.10 & 19.01 & 17.80 & 17.80 & 3.91 & 2.69 & 11.76 & -1.21 & 7.85 & 9.07 \\
\hline $\begin{array}{l}\text { Direct to } \\
\text { retailers and } \\
\text { restaurants }\end{array}$ & 32.93 & 35.03 & 23.58 & 13.56 & 2.41 & 2.10 & -9.35 & -19.37 & -19.37 & -11.45 & -21.47 & -32.62 & -10.02 & -21.17 & -11.15 \\
\hline $\begin{array}{l}\text { Direct to } \\
\text { intermediaries }\end{array}$ & -1.37 & -6.67 & -3.66 & -9.24 & 3.40 & -5.30 & -2.29 & -7.87 & -7.87 & 3.01 & -2.57 & 10.07 & -5.58 & 7.06 & 12.64 \\
\hline $\begin{array}{l}\text { Direct to } \\
\text { institutions }\end{array}$ & 3.50 & -5.59 & -4.12 & 12.72 & 3.63 & -9.09 & -7.62 & 9.21 & 9.21 & 1.47 & 18.30 & 9.22 & 16.83 & 7.74 & -9.09 \\
\hline $\begin{array}{l}\text { Unclassified } \\
\text { channels }\end{array}$ & 0.10 & -2.71 & 0.35 & 0.34 & -0.63 & -2.81 & 0.25 & 0.24 & 0.24 & 3.06 & 3.05 & 2.08 & -0.01 & -0.98 & -0.97 \\
\hline
\end{tabular}

Source: Author's calculations.

Note: Species indicators are: 1 = Average Joes; 2 = Small-scale Startups; 3 = Processors; 4 = Community Service Providers; 5 = Coops; and $6=$ Traditional Produce Warehousers. The first listed species in the column heading is the reference category. Values in bold indicate statistical significance at or beyond the 10 percent level. 
Table 4.18. Mean Differences in Species Community Service Offerings Variables: Species-to-Species Comparisons

\begin{tabular}{|c|c|c|c|c|c|c|c|c|c|c|c|c|c|c|c|}
\hline Variable & 1 vs. 2 & 1 vs. 3 & 1 vs. 4 & $\begin{array}{c}1 \text { vs. } \\
5\end{array}$ & 1 vs. 6 & 2 vs. 3 & 2 vs. 4 & 2 vs. 5 & 2 vs. 6 & 3 vs. 4 & $\begin{array}{c}3 \text { vs. } \\
5\end{array}$ & 3 vs. 6 & 4 vs. 5 & 4 vs. 6 & 5 vs. 6 \\
\hline $\begin{array}{l}\text { Total community services } \\
\text { offered }\end{array}$ & 1.25 & -0.98 & -2.36 & 1.91 & -0.11 & -2.23 & -3.62 & 0.66 & 0.66 & -1.39 & 2.89 & 0.86 & 4.27 & 2.25 & -2.02 \\
\hline $\begin{array}{l}\text { Paid employment } \\
\text { opportunities for youth }\end{array}$ & 0.28 & -0.30 & -0.16 & 0.05 & -0.30 & -0.58 & -0.44 & -0.24 & -0.24 & 0.13 & 0.34 & 0.00 & 0.21 & -0.14 & -0.35 \\
\hline Accepting SNAP benefits & 0.13 & -0.25 & -0.32 & 0.13 & -0.09 & -0.38 & -0.44 & 0.00 & 0.00 & -0.06 & 0.38 & 0.16 & 0.44 & 0.22 & -0.22 \\
\hline $\begin{array}{l}\text { Accepting WIC or FMNP } \\
\text { benefits }\end{array}$ & 0.03 & -0.17 & -0.33 & 0.02 & 0.00 & -0.20 & -0.36 & -0.01 & -0.01 & -0.16 & 0.19 & 0.17 & 0.35 & 0.33 & -0.02 \\
\hline $\begin{array}{l}\text { Matching programs for } \\
\text { SNAP benefits }\end{array}$ & 0.35 & 0.06 & 0.12 & 0.18 & 0.01 & -0.29 & -0.22 & -0.17 & -0.17 & 0.06 & 0.12 & -0.05 & 0.06 & -0.11 & -0.17 \\
\hline $\begin{array}{l}\text { Nutrition or cooking } \\
\text { education }\end{array}$ & 0.01 & -0.14 & -0.56 & 0.26 & 0.02 & -0.15 & -0.57 & 0.25 & 0.25 & -0.42 & 0.40 & 0.16 & 0.82 & 0.58 & -0.24 \\
\hline Health screenings & 0.03 & -0.07 & -0.19 & 0.03 & -0.05 & -0.11 & -0.22 & & & -0.12 & 0.11 & 0.02 & 0.22 & 0.14 & -0.08 \\
\hline $\begin{array}{l}\text { Transportation services for } \\
\text { consumers to access your } \\
\text { operation }\end{array}$ & 0.03 & -0.17 & -0.11 & 0.03 & 0.12 & -0.20 & -0.14 & 0.00 & 0.00 & 0.06 & 0.20 & 0.29 & 0.14 & 0.22 & 0.08 \\
\hline Operating a mobile market & 0.13 & -0.12 & -0.06 & 0.38 & 0.16 & -0.25 & -0.19 & 0.25 & 0.25 & 0.06 & 0.50 & 0.28 & 0.44 & 0.22 & -0.22 \\
\hline Subsidized farm shares & 0.11 & -0.09 & 0.08 & 0.03 & -0.14 & -0.20 & -0.03 & -0.08 & -0.08 & 0.17 & 0.12 & -0.05 & -0.06 & -0.22 & -0.17 \\
\hline $\begin{array}{l}\text { Education about community } \\
\text { and food systems issues }\end{array}$ & -0.08 & 0.11 & -0.16 & 0.37 & 0.01 & 0.19 & -0.07 & 0.46 & 0.46 & -0.26 & 0.27 & -0.10 & 0.53 & 0.17 & -0.36 \\
\hline $\begin{array}{l}\text { Education for programs in } \\
\text { community or school } \\
\text { gardening }\end{array}$ & -0.06 & -0.04 & -0.34 & 0.14 & 0.02 & 0.03 & -0.28 & 0.21 & 0.21 & -0.30 & 0.18 & 0.06 & 0.48 & 0.36 & -0.12 \\
\hline $\begin{array}{l}\text { Food donation to local food } \\
\text { pantries and banks }\end{array}$ & 0.25 & 0.02 & 0.19 & 0.15 & 0.05 & -0.23 & -0.06 & -0.11 & -0.11 & 0.17 & 0.12 & 0.03 & -0.05 & -0.14 & -0.09 \\
\hline $\begin{array}{l}\text { Other community services or } \\
\text { activities }\end{array}$ & 0.25 & -0.11 & -0.21 & 0.21 & 0.01 & -0.36 & -0.46 & -0.04 & -0.04 & -0.10 & 0.32 & 0.12 & 0.42 & 0.22 & -0.20 \\
\hline
\end{tabular}

Source: Author's calculations.

Note: Species indicators are: 1 = Average Joes; 2 = Small-scale Startups; 3 = Processors; 4 = Community Service Providers; 5 = Coops; and $6=$ Traditional Produce Warehousers.

The first listed species in the column heading is the reference category. Values in bold indicate statistical significance at or beyond the 10 percent level. FMNP = Farmers Market

Nutrition Program; SNAP = Supplemental Nutrition Assistance Program; and WIC = Women Infants and Children (program). 
Table 4.19. Mean Differences in Species' Morphological Variables: Species-to-Species Comparisons

\begin{tabular}{|c|c|c|c|c|c|c|c|c|}
\hline Variable & 1 vs. 2 & 1 vs. 3 & 1 vs. 4 & 1 vs. 5 & 1 vs. 6 & 2 vs. 3 & 2 vs. 4 & 2 vs. 5 \\
\hline Profit motivated & 0.86 & 0.27 & 0.33 & 0.34 & 0.39 & -0.59 & -0.53 & -0.53 \\
\hline Cooperative form & -0.14 & -0.11 & -0.44 & & -0.17 & 0.04 & -0.30 & -0.86 \\
\hline Firm age $(\ln )$ & 0.35 & 0.02 & -0.72 & -0.49 & -0.46 & -0.33 & -1.07 & -0.84 \\
\hline Acts as broker & -0.36 & -0.35 & -1.05 & -0.54 & 0.20 & 0.01 & -0.69 & -0.18 \\
\hline Nonsales percentage of revenue & 0.01 & -0.22 & -0.30 & 0.02 & 0.01 & -0.23 & -0.31 & 0.01 \\
\hline Total warehouse space & 2539.78 & -11099.88 & -16643.49 & -2378.94 & -17720.94 & -13639.66 & -19183.27 & -4918.71 \\
\hline Number of delivery vehicles & 1.75 & -2.90 & 0.80 & 0.76 & -5.70 & -4.65 & -0.95 & -0.99 \\
\hline Offer transportation services to producers & & & 0.44 & 0.29 & 0.17 & & -0.56 & -0.71 \\
\hline Additional packaging involvement & -0.26 & -0.79 & -0.24 & 0.61 & 0.06 & -0.53 & 0.02 & $\mathbf{0 . 8 7}$ \\
\hline Processing facilities & -0.14 & -0.89 & -0.67 & & -0.17 & -0.75 & -0.52 & 0.14 \\
\hline Online ordering system & -0.08 & -0.12 & 0.23 & -0.08 & -0.10 & -0.04 & 0.31 & 0.00 \\
\hline Rental space for others & & -0.11 & & & & -0.11 & & \\
\hline Retail space for the hub & 0.14 & 0.07 & -0.72 & 0.28 & 0.28 & -0.07 & -0.86 & 0.14 \\
\hline Licensed shared use kitchen & -0.04 & -0.23 & -0.64 & 0.03 & 0.03 & -0.19 & -0.60 & 0.07 \\
\hline & 2 vs. 6 & 3 vs. 4 & 3 vs. 5 & 3 vs. 6 & 4 vs. 5 & 4 vs. 6 & 5 vs. 6 & \\
\hline Profit motivated & -0.48 & 0.06 & 0.07 & 0.11 & 0.01 & 0.06 & 0.05 & \\
\hline Cooperative form & -0.02 & -0.34 & -0.89 & -0.06 & -0.56 & 0.28 & $\mathbf{0 . 8 3}$ & \\
\hline Firm age $(\ln )$ & -0.82 & -0.74 & -0.51 & -0.48 & 0.23 & 0.26 & 0.03 & \\
\hline Acts as broker & 0.56 & -0.70 & -0.19 & 0.55 & 0.51 & $\mathbf{1 . 2 5}$ & 0.74 & \\
\hline Nonsales percentage of revenue & -0.01 & -0.08 & 0.24 & 0.23 & 0.32 & 0.31 & -0.01 & \\
\hline Total warehouse space & -20260.71 & -5543.61 & 8720.95 & -6621.05 & 14264.56 & -1077.44 & -15342.00 & \\
\hline Number of delivery vehicles & -7.45 & 3.70 & 3.66 & -2.80 & -0.04 & -6.50 & -6.46 & \\
\hline Offer transport services to producers & $-\mathbf{0 . 8 3}$ & 0.44 & 0.29 & 0.17 & -0.15 & -0.28 & -0.13 & \\
\hline Additional packaging involvement & 0.32 & 0.55 & 1.40 & 0.86 & 0.85 & 0.31 & -0.54 & \\
\hline Processing facilities & -0.02 & 0.23 & 0.89 & 0.73 & 0.67 & 0.50 & -0.17 & \\
\hline Online ordering system & -0.02 & 0.35 & 0.04 & 0.02 & -0.31 & -0.33 & -0.02 & \\
\hline Rental space for others & & -0.89 & 0.11 & -0.89 & & & & \\
\hline Retail space for the hub & 0.14 & -0.79 & 0.21 & 0.21 & & & & \\
\hline Licensed shared use kitchen & 0.07 & -0.40 & 0.26 & 0.26 & 0.67 & 0.67 & & \\
\hline
\end{tabular}

Source: Author's calculations.

Note: Species indicators are: 1 = Average Joes; 2 = Small-scale Startups; 3 = Processors; 4 = Community Service Providers; $5=$ Coops; and $6=$ Traditional Produce Warehousers. The first listed species in the column heading is the reference category. Values in bold indicate statistical significance at or beyond the 10 percent level. Black cells indicate no value could be calculated. 
Table 4.20 Regression Analysis Results for Validation Stage: Channel Reliance and Community Services

\begin{tabular}{|c|c|c|c|c|c|c|c|c|c|c|c|c|c|c|c|c|}
\hline & & \multicolumn{3}{|c|}{ Direct to Consumers } & \multicolumn{3}{|c|}{ Direct to Intermediaries } & \multicolumn{3}{|c|}{$\begin{array}{c}\text { Direct to Retailers and } \\
\text { Restaurants }\end{array}$} & \multicolumn{3}{|c|}{ Direct to Institutions } & \multicolumn{3}{|c|}{$\begin{array}{c}\text { Number of } \\
\text { Community Services }\end{array}$} \\
\hline \multicolumn{2}{|c|}{ Intercept } & $29.0^{* * *}$ & $27.6^{* * *}$ & $49.1^{* * *}$ & 4.3 & 5.0 & -8.8 & $53.0^{* * *}$ & $52.2^{* * *}$ & $32.4^{*}$ & $13.4^{* * *}$ & $13.7^{* * *}$ & $25.1^{*}$ & $4.8^{* * *}$ & $5.1^{* * *}$ & $5.9^{* * *}$ \\
\hline \multirow{5}{*}{$\begin{array}{l}\frac{n}{0} \\
\frac{0}{0} \\
\stackrel{0}{n}\end{array}$} & SSSs & $35.0^{* *}$ & $36.1^{* *}$ & $31.1^{* *}$ & 1.4 & 3.5 & 6.1 & $-32.9^{* * *}$ & $-37.3^{* * *}$ & $-33.0^{* *}$ & -3.5 & -2.8 & -4.5 & -1.1 & -1.1 & -1.2 \\
\hline & Proc. & 20.0 & 20.5 & 15.5 & 6.7 & 6.9 & 9.3 & $-35.0^{* * *}$ & $-35.4^{* * *}$ & $-32.2^{* * *}$ & 5.6 & 5.4 & 4.7 & $1.6^{*}$ & $1.4^{*}$ & $1.6^{* *}$ \\
\hline & CSPs & 16.0 & 26.9 & 27.3 & 3.7 & 4.9 & 4.9 & -23.6 & -22.8 & -23.7 & 4.1 & -8.6 & -8.1 & $3.4^{* * *}$ & $3.5^{* * *}$ & $3.7^{* * *}$ \\
\hline & Coops & 17.0 & 16.9 & 15.4 & 9.2 & 8.9 & $10.4 *$ & -13.6 & -14.5 & -13.3 & $-12.7^{*}$ & $-10.8^{*}$ & $-11.9^{* *}$ & $-1.8^{*}$ & $-2.0^{* * *}$ & $-2.0^{* * *}$ \\
\hline & TPWs & 8.0 & 10.2 & 9.8 & -3.4 & -2.5 & -1.6 & -2.4 & -5.8 & -5.6 & -3.6 & -3.2 & -3.8 & 0.3 & 0.2 & 0.3 \\
\hline \multirow{6}{*}{$\begin{array}{l}\stackrel{\Xi}{.0} \\
.00 \\
\mathscr{0} \\
\approx\end{array}$} & SW & & 11.1 & 11.7 & & -2.0 & -1.5 & & 3.5 & 2.7 & & -10.3 & $-10.6^{*}$ & & -0.2 & -0.1 \\
\hline & NW & & -15.6 & -16.6 & & -7.1 & -5.8 & & 18.5 & 19.3 & & 6.4 & 5.4 & & -0.5 & -0.5 \\
\hline & MW & & -8.1 & -9.4 & & -0.7 & 0.7 & & -0.2 & 0.7 & & 10.5 & 9.7 & & -1.3 & -1.2 \\
\hline & GU & & -23.5 & -17.5 & & -1.3 & -4.1 & & -18.5 & -24.3 & & $45.1^{* * *}$ & $47.5^{* * *}$ & & -0.3 & 0.0 \\
\hline & SE & & 8.5 & 2.1 & & -4.2 & -2.2 & & 8.3 & 12.4 & & $-10.6^{*}$ & -10.3 & & -0.9 & -0.7 \\
\hline & GL & & 5.9 & 5.4 & & 4.1 & 4.9 & & -3.3 & -3.2 & & -5.9 & -6.4 & & 1.2 & 1.3 \\
\hline \multicolumn{2}{|c|}{ Mgr. age } & & & -0.4 & & & 0.2 & & & 0.4 & & & -0.2 & & & 0.0 \\
\hline \multicolumn{2}{|c|}{$\begin{array}{c}\text { Adjusted } \\
R^{2}\end{array}$} & 0.03 & 0.01 & -0.01 & -0.00 & -0.05 & -0.04 & 0.11 & 0.087 & 0.08 & 0.02 & 0.26 & 0.27 & 0.22 & 0.25 & 0.25 \\
\hline
\end{tabular}

Source: Author's calculations.

Note: The reference group for species is Average Joes; the reference group for region is Northeast. Statistical significance: $* 10 \%$, ** $5 \%$, and $* * * 1 \%$. Species indicators: SSSs $=$

Small-scale Startups; Proc. = Processors; CSPs = Community Service Providers; TPWs $=$ Traditional Produce Warehousers. Region indicators: SW = Southwest; NW =

Northwest; $\mathrm{MW}=$ Midwest; GU = Gulf states; $\mathrm{SE}=$ Southeast; GL $=$ Great Lakes. Mgr. age = age of highest-ranking manager. 
Table 4.21 Regression Analysis Results for Validation Stage: Product Reliance in Primary Product Categories

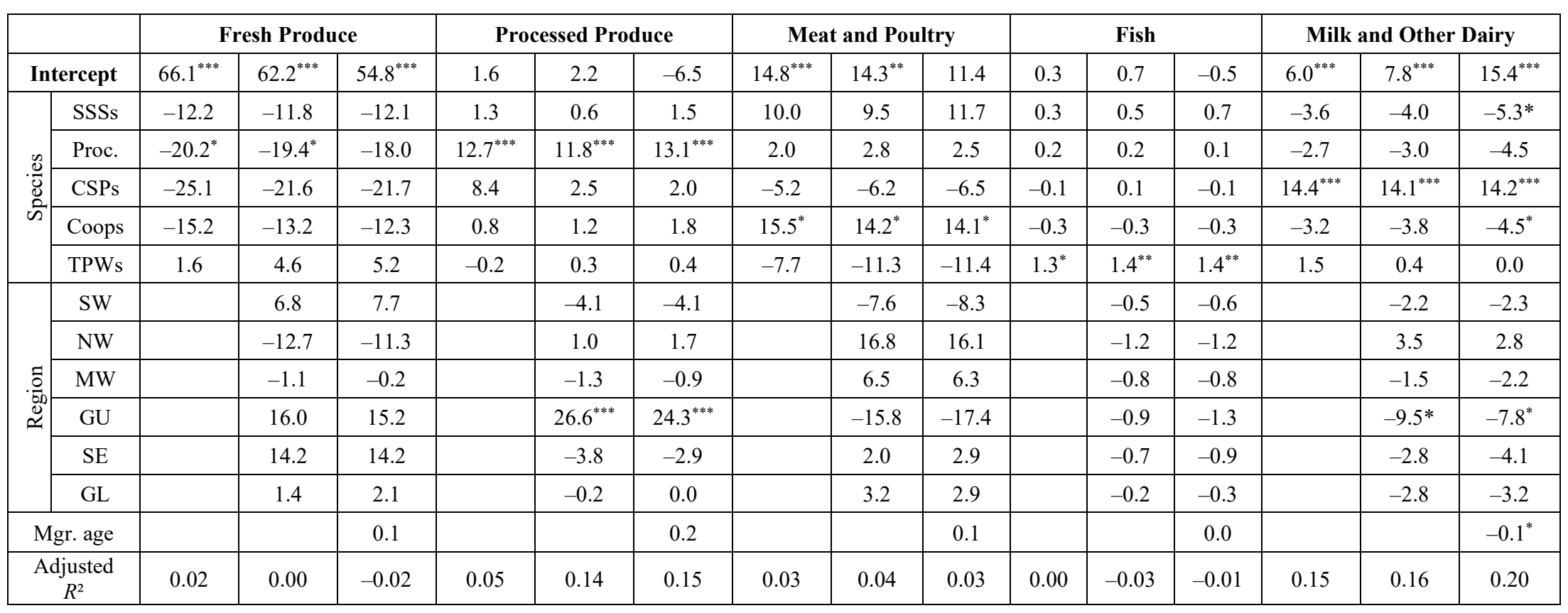

Source: Author's calculations.

Note: The reference group for species is Average Joes; the reference group for region is Northeast. Statistical significance: $* 10 \%$, $* * 5 \%$, and $* * * 1 \%$. Species indicators: SSSs $=$ Small-scale Startups; Proc. = Processors; CSPs = Community Service Providers; TPWs = Traditional Produce Warehousers. Region indicators: SW = Southwest; NW =

Northwest; $\mathrm{MW}=$ Midwest; $\mathrm{GU}=$ Gulf states; $\mathrm{SE}=$ Southeast; $\mathrm{GL}=$ Great Lakes. Mgr. age = age of highest-ranking manager. 
Table 4.22 Regression Analysis Results for Validation Stage: Product Reliance in Secondary Product Categories

\begin{tabular}{|c|c|c|c|c|c|c|c|c|c|c|c|c|c|c|c|c|}
\hline & & \multicolumn{3}{|c|}{ Eggs } & \multicolumn{3}{|c|}{ Dry Goods } & \multicolumn{3}{|c|}{ Baked Goods } & \multicolumn{3}{|c|}{ Coffee and Tea } & \multicolumn{3}{|c|}{ Other Value Added } \\
\hline \multicolumn{2}{|c|}{ Intercept } & $3.3^{* * *}$ & $2.8^{*}$ & $7.9^{* * *}$ & 1.5 & 0.3 & 6.3 & $1.0^{*}$ & $1.7^{*}$ & $3.9^{*}$ & 0.2 & 0.2 & -0.1 & 3.3 & $6.2^{*}$ & 9.0 \\
\hline \multirow{5}{*}{$\begin{array}{l}0 \\
\frac{0}{0} \\
\tilde{0} \\
\text { की }\end{array}$} & SSSs & -0.6 & -1.0 & -1.8 & $6.5^{*}$ & $6.6^{*}$ & 6.3 & 0.8 & 1.2 & 0.4 & 0.0 & -0.1 & 0.0 & -2.2 & -1.4 & -2.0 \\
\hline & Proc. & -0.2 & -0.1 & -0.8 & 0.1 & 0.3 & -0.3 & 0.8 & 0.8 & 0.5 & 0.1 & 0.1 & 0.1 & $7.7^{*}$ & $7.2^{*}$ & $7.3^{*}$ \\
\hline & CSPs & 2.7 & 2.2 & 2.5 & -0.1 & 1.5 & 2.1 & 0.5 & 0.8 & 0.8 & $1.2^{* * *}$ & $1.2^{* * *}$ & $1.2^{* *}$ & 0.2 & 1.1 & 1.1 \\
\hline & Coops & 0.5 & 0.5 & 0.1 & -0.9 & -0.6 & -0.9 & 0.8 & 0.7 & 0.5 & $0.5^{*}$ & $0.5^{*}$ & $0.5^{*}$ & -1.7 & -2.2 & -2.6 \\
\hline & TPWs & 0.7 & 0.3 & 0.2 & 1.0 & 1.6 & 1.5 & -0.9 & -0.8 & -0.9 & -0.2 & -0.2 & -0.2 & 2.0 & 2.4 & 2.2 \\
\hline \multirow{6}{*}{ 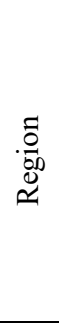 } & SW & & 1.2 & 1.2 & & $6.1^{*}$ & 6.1 & & -1.0 & -0.9 & & 0.2 & 0.2 & & -1.8 & -2.0 \\
\hline & NW & & 3.3 & 2.9 & & -1.7 & -2.2 & & -1.7 & -1.7 & & 0.4 & 0.4 & & -5.7 & -6.0 \\
\hline & MW & & 2.3 & 2.0 & & 2.1 & 2.0 & & -1.0 & -1.2 & & 0.1 & 0.1 & & -5.1 & -5.4 \\
\hline & GU & & -0.5 & 0.7 & & -2.4 & -0.8 & & -2.4 & -1.8 & & -0.5 & -0.6 & & -7.9 & -7.4 \\
\hline & SE & & -0.2 & -0.7 & & -0.4 & -0.5 & & -0.7 & -1.2 & & 0.0 & -0.1 & & -5.9 & -5.5 \\
\hline & GL & & -0.4 & -0.5 & & 0.6 & 0.5 & & -0.6 & -0.7 & & 0.0 & 0.0 & & -1.8 & -2.0 \\
\hline \multicolumn{2}{|c|}{ Mgr. age } & & & $-0.1^{* *}$ & & & -0.1 & & & 0.0 & & & 0.0 & & & -0.1 \\
\hline \multicolumn{2}{|c|}{ Adjusted $R^{2}$} & -0.03 & -0.04 & 0.01 & 0.00 & -0.02 & -0.01 & -0.01 & -0.03 & -0.01 & 0.08 & 0.04 & 0.03 & 0.02 & -0.01 & -0.03 \\
\hline
\end{tabular}

Source: Author's calculations.

Note: The reference group for species is Average Joes; the reference group for region is Northeast. Statistical significance: * $10 \%$, ** $5 \%$, and $* * * 1 \%$. Species indicators: SSSs $=$ Small-scale Startups; Proc. $=$ Processors; CSPs $=$ Community Service Providers; TPWs $=$ Traditional Produce Warehousers. Region indicators: SW $=$ Southwest; NW $=$

Northwest; $\mathrm{MW}=$ Midwest; GU $=$ Gulf states; $\mathrm{SE}=$ Southeast; $\mathrm{GL}=$ Great Lakes. Mgr. age $=$ age of highest-ranking manager 


\section{CHAPTER 5}

\section{THE ECOLOGY OF FOOD HUB ORGANIZATIONS: IMPLICATIONS FOR POLICY ON SCALING UP LOCAL AND REGIONAL FOOD SYSTEMS}

\subsection{Introduction}

In Chapter 4, I applied the organizational species concept (OSC) and attendant cluster analysis methodology to investigate the homeostatic property clusters (HPCs) of US food hub organizations (FHOs). This operationalization of the OSC identified a set of organizational kinds based on patterns of HPCs and following the validation stage I arrived at a clustering solution with six partitions as a set of FHO species. Whereas Chapter 4 outlined the morphological features and strategic behaviors of the six species, here I discuss implications for policy and management of local and regional food systems (LRFSs), especially their scaling up. I investigate the differences in key performance, financial, and supplier elements (for example, total value of products, dependency on grants, number of minority-operated vendors) by performing a group analysis of variance and a matrix of bilateral means testing using independent-samples $t$ tests. Also, I use regression analysis to investigate performance differences while accounting for exogenous elements.

These investigations are intended to understand the role of different species in the econosystems they inhabit. Part of this role is identified through the validation of the HPC-based kinds as species using strategic orientation information. Organizational niches, as I operationalize them in outbound strategy space, represent the throughput value chains of the species as well as how they perform their identity of having a "commitment to place" and thus enact the alterity of the local foods movement (LRFM). I focus on a few elements key to addressing the scaling-up challenge: (1) the numbers 
and types of suppliers that are symbiotic with these coordinating intermediaries; (2) the relative scale and survivability of different species and the contribution these species make to increasing the total scale of food systems; and (3) metrics of population "health" for each of the species, on average. Items (1) and (2) specifically target the two facets identified in the literature on scaling-up LRFSs, whereas item (3) allows policymakers and practitioners to target interventions to improve or stimulate specific types of FHOs suited to the environmental conditions faced in their pertinent foodshed. Results indicate that different species of FHOs suit different contexts and goals.

Certain patterns are important to note in the morphological and strategic profiles of the different species. For example, the Small-scale Startups proto-member ${ }^{76}$ shows a morphological profile that implies relative undercapitalization, which is not surprising given their lower mean age. This also matches their strategic niche, which highly relies on direct-to-consumer (DTC) channels. It is intriguing given that they have the secondhighest (after Coops) mean reliance on meat and poultry, which generally has higher relative asset needs for cold chains. In contrast, Community Service Providers (CSPs) have many capital-intensive features, but, overall, their footprint (in warehouse square footage) is small. On the basis of their strategic orientation, CSPs likely use these assets for intensive community engagement programs, whereas the product and channel strategy of the CSP proto-member is highly diversified. Coops are consistently below average for community service offerings, implying that they are likely to enact alterity through means not captured by the 2015 National Food Hub Survey; a best estimate is that Coops enact

\footnotetext{
${ }^{76}$ The proto-member is the abstract representation of the species, based on the average characteristics of the species population based on the sample.
} 
the social embeddedness of a commitment to place by transmitting information about provenance to consumers while serving the economic and social needs of producers.

Overall, FHOs are largely characterized by how much they rely on fresh produce sold to DTC channels, followed by direct-to-retail (DTR) — especially restaurants and small grocers. This raises substantial questions about how FHOs perform in scaling up LRFSs through high-volume channels and product diversification. FHOs still likely enact the functions of a coordinating intermediary, perhaps filling a new economic role that other arrangements such as farmers' markets and community-supported agriculture programs may have historically filled. This question and the general efficiency implications are worth investigating further. Also, some species fill specific roles in food distribution systems. The fact that Coops, by example, on average rely on meat and poultry products the most fits neatly with the theoretical expectation that cooperative organizations arise in places where markets - as is often the case for abattoir and animal processing services at small scales - are thin. It would not be surprising if CSPs tended to live in urban settings and food deserts, filling roles as food social work organizations. To preserve the confidentiality of responses from the primary data collectors, detailed geographic data were unavailable from the 2015 National Food Hub Survey.

\subsection{Results}

I discuss four thematic areas in the sections that follow: the number and types of suppliers used and supplier demographics; managerial and financial issues, including manager experience in key functions, startup funding and dependence on grants, and cost structures; the extent to which the FHO's mission and daily operations relate to certain social values; and measures of firm performance through total value of product moved (TVPM) through the hub and estimated profit. 
Tables 5.1 and 5.2 present the mean differences resulting from independentsamples $t$ test analysis comparing the cases in a given species to the group of all other cases in the final sample. These results provide a reference of differences, and, most important, a pertinent statistical test; bolded mean differences had $p$ values for the relevant $t$ statistic $^{77}$ that were at or beyond the 10 percent level. Regarding the sign on mean difference values in the tables, the reference group is the collection of all in-sample cases not of the given species (in the column) and thus negative signs indicate that the mean of the reference group is less than the mean for the species.

\subsubsection{Suppliers}

The makeup of FHO suppliers is relevant to increasing scale, transactions costs regimes, and social equity in distribution channel arrangements. Figure 5.1 presents trend line-style mean profiles for dichotomous variables on the types of suppliers used by FHOs, and Figure 5.3 presents demographics in terms of the percentage of suppliers that are owned or operated by socially disadvantaged persons (women and people of color) or that are new and beginning firms (in operation for fewer than 10 years). Figures 5.2 and 5.4 present data in an error bar format, with 95 percent confidence intervals. Across species, more 80 percent of FHOs source from primary agricultural producers (farms or ranches), and of those that do the majority of their suppliers are of this type. The overall mix of supplier types across species does vary, however.

Small-scale Startups primarily source from farms and food processors. They are substantially less likely to include among their supplier portfolio a different food

\footnotetext{
${ }^{77}$ First an $F$-test is conducted to make statistical inference about the equality of variances between the two populations in the comparison. If the results of the $F$-test indicate we are justified in concluding the two populations have equal variances, then the results of a equal variance $t$-test are the relevant metric, otherwise the results of the different variances $t$-test are used.
} 
distributor (26 percent less likely) or their own operations ( 28 percent less likely), which as the mean differences are statistically significant. Sourcing of products from the food hub's own operations is most prevalent among Processors. Meanwhile, CSPs have approximately the same proportion of cases using food processors as farms, and their mean difference in the incidence of using external food processors is statistically significant; on average, they are at least 29 percent more likely to source from an external food processor than all other cases. CSPs are also the most likely of any species to purchase from nonfood businesses and suppliers of unclassified type. Traditional Produce Warehousers (TPWs) are most likely to purchase from other distributors. This implies that CSPs and TPWs are likely engaged in extended (less proximal) transactions.

It is intriguing that Coops, along with CSPs, have the lowest average proportion of cases indicating that they source from farms and ranches, although this is still exceptionally high at nearly 90 percent of cases using the type. This trend of the Coops proto-member being below the total average for all FHOs continues for incidences of external food processor and distributor supplier types. Without information on the proportion of products received from each supplier type — by volume or, as with channel reliance, value - it is hard to tell what drives the incidences. Do individual cases trade-off between supplier types? Do they take a portfolio approach? Or do they tend to specialize in sourcing from one or two types? Answers to these questions would provide a better understanding of the upstream connections of FHOs and elucidate their role as intermediaries in LRFSs. Unfortunately, data to address such topics are not collected in the 2015 National Food Hub Survey. 
However, some supplier demographic data are available in the 2015 National Food Hub Survey to help understand the upstream connections of FHO species. In my work, I have investigated four data points - the only usable elements of the 2015 National Food Hub Survey related to demographics. Two of these relate to the role of FHO species in enhancing the equity of LRFSs for supplying enterprises owned by persons in marginalized groups. The other two relate more directly to the agriculture of the middle $(\mathrm{AOTM}){ }^{78}$ approach to the scaling-up challenge. On average, FHOs in the subsample use a maximum of 78 suppliers, but these numbers change dramatically by species. The proto-member for TPWs has an average of 213 reported suppliers, followed by Processors at 93. Average Joes and Coops have around 50 suppliers on average, whereas Small-scale Startups and CSPs have around 30. FHOs of a given species may have a different effect on the number of suppliers they are able to connect to markets for proximal, source-identified products.

LRFSs are targeted as a means for including more underrepresented minoritiesor, as the US Department of Agriculture refers to them, "socially disadvantaged"enterprises in food systems. Overall, FHOs in the sample are doing poorly at inclusion of suppliers owned and operated by women (30 percent of suppliers) and people of color (20 percent of suppliers). Unfortunately, we cannot confidently infer how species populations diverge in the percentage of suppliers owned and operated by women; none of the observed mean differences were statistically significant at or beyond the 10 percent level. However, it is not surprising because, as Figures 5.3 and 5.4 show, the proto-members for

\footnotetext{
${ }^{78}$ The 2015 National Food Hub Survey included two questions - number 6.13 and 6.14-regarding small and medium farms and ranches specifically. However, both items were omitted from analysis over concerns with response validity.
} 
all species lie in a narrow band between 25 and 40 percent of reported suppliers owned and operated by women.

There is greater divergence between species for suppliers of color. Coops have the lowest average share of suppliers of color, at 4.3 percent for the proto-member, whereas CSPs have the highest average at 53.33 percent. Given the results of the independentsamples $t$ test (Table 5.1), we can confidently predict that, on average, Coops have at least 17.67 percent fewer people of color suppliers, compared with all other cases and that CSPs include at least 35.29 percent more of firms owned and operated by people of color among their suppliers than all other cases of FHOs. However, the 95 percent confidence interval for the mean percentage of people of color supplying to CSPs spans all possible values; this is likely because of a small population size for the species compounded by a low response rate among CSP cases for this question. The overall average for the combined sample of all FHO cases is 18.41 percent.

For new and beginning firms, the numbers are better, with all species except Processors having approximately 50 percent or more of their suppliers in operation for less than 10 years; average values range from 47.74 percent for Average Joes to 61.2 percent for CSPs. For Processors, this number is 32.75 percent, and we are confident that the mean difference for this species is at least 20.84 percent lower than in all other cases. Mean spreads, represented by the 95 percent confidence intervals in Figure 5.4 are relatively large (and for CSPs again run the entire range of possible values). This is likely because of variance and response rates within species, as well as measurement error given that the values here are self-reported percentages by FHO manager-respondents. 
However, on the basis of observed means, most FHO species perform approximately the same in providing marketing channels for new ventures.

The majority of FHO suppliers are primary agricultural producers, although with species slightly varying. The TPW proto-member has primary agricultural producers that account for 77.11 percent of suppliers, whereas for the CSP proto-member, this is 91.76 percent. The other species fall within this range. None of the species has a statistically significant mean difference compared with all other cases. Results in Figure 5.4, however, show that approximately 85 percent of suppliers to the Processor species protomember are farms and ranches, as its 95 percent confidence interval is relatively tight. Similarly, TPWs fall below Processors. The status of CSPs is, as usual, difficult to conclude.

\subsubsection{Managerial and Financial}

Understanding the managerial and financial positioning of FHO species is important for policy interventions to strengthen or expand through new establishments of the population of a given species. Targeting one or more species may be especially noteworthy if policymakers identify specific gaps in the foodsheds they manage. To provide some useful insight in crafted target policies for this ecosystem management approach, I consider the reported levels of manager experience in key FHO functions among the species (Figures 5.5 and 5.6), the sources of their founding capital/initial funding (Figures 5.7 and 5.8), and their cost structures (Figures 5.9 and 5.10). Table 5.2

reports results of independent-samples $t$ test analysis for startup funding sources and cost structures.

Here, managerial experience in key functions was reported on a 6-point Likerttype scale ranging from 1 ("no experience") to 6 ("10 or more years of experience"). In 
Figure 5.6, the solid black line denotes the midpoint value in the scale; values to the right of this line indicate that the senior manager has 3 or more years of experience in that function. Mean spreads tend to be wide across each of the key functions and for all species, making definitive conclusions about inferences to the population difficult.

Figure 5.5 shows that the most senior manager of the Coops proto-member has the fewest years of experience compared with the sample average in each of the FHO key functional areas. This is surprising given that Coops are, on average, older organizations, and their most senior managers have nearly the sample average in terms of age and education level. The only key function for which Coops have a statistically significant mean difference, however, is for other miscellaneous functional skills; that mean difference is 1.22 lower than the mean for all other species. The most experienced managers across key functions have consistently been for CSPs; the proto-member for this species is the oldest in firm age and has the eldest and highest educated manager. The mean differences for functions are not statistically significant for this species but are statistically significant for education level of the senior manager. Processors have the highest average experience in agricultural production but have statistically significant mean differences - both of substantial magnitude and both lower than the average for all other cases - for experience in warehousing and management. Meanwhile, TPW managers are rated highest in experience with warehousing, strategic planning, and other unclassified primary functions. The first two have statistically significant mean differences compared with other cases, both positive and of moderate magnitudes.

The results in Figure 5.5 indicate a number of realms for potential policy interventions. FHO managers across species — including processors - appear to have low 
levels of experience in processing functions, which are key elements of value addition. Coops managers have lower levels of experience compared with other FHO species and may be a key group to target with training and mentorship programs. Managers of Smallscale Startups substantially lack experience in warehousing and management, thus programs starting a new FHO would benefit from prioritizing these areas. These results indicate that TPW managers lack experience in agricultural production; given that they rely highly on fresh produce, this will be a critical need given new food safety regulations and their relative total size.

Figure 5.7 considers the source of initial startup funds. Data are limited to a dichotomous series. Here, TPWs have extremely large mean spreads at the 95 percent confidence interval; this is due to low response rates for this data series from cases of this species. Coops are the most likely (see Table 5.2) to have relied on membership fees when they were founded. Coops, along with Small-Scale Startups, are the only two species with less than 50 percent likelihood, on average, to have founder capital involved in FHO establishment. TPWs have the most diversified startup sources with the highest average (at 4.33 different sources of startup funds). Those most likely in the mix are internal program transfers, foundation grants, individual donations, and founder capital. It is interesting that TPWs have effectively no borrowing from creditors (such as private lenders or banks). The Processor proto-member is the second highest (mean, 3.58), followed by Coops (mean, 3.5), whereas Average Joes, Small-scale Startups, and CSPs all sit near averages of three total sources. Complete profiles for these other species cannot be clearly delineated; only the most likely source of initial funds can be identified. For Coops, this is membership fees; for Processors, Average Joes, and CSPs, it is founder 
capital; and for Small-scale Startups, either membership fees or individual donations. CSPs reported no use of local government funds, and only moderate use of federal and state funds and foundation grants. Founder's capital is the only source with a majority use across overall.

Two elements of current funding sources are worth noting in addition to the startup funding. I used one of these - the percentage of revenue from nonsales - as a morphological variable and reported the other-a rating of how dependent the FHO is on grants-in Table 5.2. Coops' proto-member is the lowest on both measures, indicating that they are the most financially autonomous. Meanwhile CSPs, followed by Processors, have the highest average reliance on nonsales revenues, at 44 percent and 36 percent, respectively. However, CSPs depend on grants much less than the sample average, whereas Processors' dependency is the second highest, which is slightly counterintuitive. Small-scale Startups depend the most on grants, with TPWs also above the sample average. However, these are fine-grained differences as even the highest species' mean is 0.77 on a 3-point Likert-type scale ranging from 0 ("not at all dependent") to 2 ("highly dependent"). More striking and worthy of future investigation is the source of nonsales revenues for the different species, if it is not grants. If Processors achieve high nonsales revenue but only achieve moderate brokerage activity, little to no facilities rental, and few grants, where is nonsales revenue sourced? Do CSPs gain rents from social services provision? Do TPWs, which offer rental space to other firms, produce so much additional volume that the total revenue in percentage from nonsales activities eclipses this? Current data cannot answer these and other questions but offer insight on how operations are structured in species to capture economic rents. 
Through cost data, the 2015 National Food Hub Survey provides insight on internal structures. Figures 5.9 and 5.10 present these structures as the mean profiles of the percentage share for several expense categories of total costs. The mean differences that are reported in Table 5.2 are not discussed at length here because the only values with statistical significance represent relatively inconsequential magnitudes. Although it is not statistically significant, the most substantial mean difference in Table 5.2 comes from comparing the CSPs in percentage of expenses to product purchases; for the sample only, the CSP proto-member has 22 percent fewer expenses to product purchases compared with all other cases.

CSPs also have the most distinctive mixture of expenses. For most categories, their proto-member is significantly higher than the sample average. This includes nearly 10 percent of expenses for facilities servicing and roughly 6 percent for utilities, both double the sample average. This is copasetic to their observed morphological structure. For all other species, cost structures of the proto-member show only small differences. Small-scale Startups have the second-highest mean percentage to facilities payments, despite morphology indicating low capitalization. One possible explanation is the higherthan-average payments on their few facilities given their young age and relatively low expenditures on other operations items (including those related to vehicles). Coops have higher consulting services expenses as a percentage but very few related to facilities or packaging; the consulting services may indicate that Coops make up for lower managerial experience through knowledge procurement on an ad hoc basis. The majority of expenses for all species are for labor. 


\subsubsection{Mission and Daily Operations}

Given the conceptual framework developed in Chapter 2 of the LRFM and LRFSs, and the definitional elements of FHOs discussed in Chapter 4 that ground them in this movement and these systems, we are also interested in the social values that different FHO species hold and enact. In Chapter 4, I considered the provision of services as a strategic orientation of the FHO species as part of the validation phase in operationalizing the OSC. I chose that series because each is a specific, discrete, measurable direct action — with specific associated costs—of each FHO case. In addition, the data underlying the variables for the community services series used there presented a number of preferential elements such as response rates, variable scale, and others. However, the 2015 National Food Hub Survey also includes a secondary series that offers a more general view of FHO orientations to social values. Unlike the community services series, this social values series records the responding manager's perception-relayed on a 3point scale — of the degree to which their FHO is engaged in a series of 11 different values-based orientations. The series is presented twice, once asking how closely the FHO's stated mission relates to each, and once asking how much their day-to-day operations reflect each value.

Figure 5.11 presents the mean profiles of the six species, plus the sample average. Here, I take a different approach to represent these data graphically so that comparisons between mission and operations can more readily be made. In previous mean profile graphics, I presented all data points as "trend lines," similar to parallel coordinates plots. In Figure 5.11, species data are presented as clustered bar graphs, with the sample average (that is, "total") presented with the same black dashed trendline as before. Figures 5.12 and 5.13 present the error bar graphs, with 95 percent confidence intervals, 
for mission and operations mean profiles, respectively. For comparison, the black line shows the midpoint value of the 3-point scale. Last, Table 5.3 presents the mean differences for each species compared with all other in-sample cases not of that species. Here, bolded values also indicate statistical significance at or beyond the 10 percent level. Results indicate that, overall, US food hubs predominantly focus on providing market access for small and medium enterprises and providing fair prices. Given my findings in section 5.2.1 that the majority of such supplying enterprises are primary agricultural producers, this implies that most FHOs are oriented in their missions and operations on the two key elements of the AOTM paradigm. Table 5.3 shows several possible inferences on the differences in these two realms between FHO species. Most notable is that the drop in relevance of mission to SME market access for Processors seen in Figure 5.11 is statistically significant, with a mean difference of 0.29 . Meanwhile, the missions of Small-scale Startups and CSPs are slightly more relevant to this social value than are other cases, whereas for Average Joes the manager ratings of relevance for their FHO's mission to providing suppliers fair prices is slightly greater than all other cases.

Trends initially indicated in previous sections related to social justice in food access for marginalized and disadvantaged populations continue here. The CSP protomember has the highest rating for the provision of food access to racial minorities being relevant to its mission and daily operations. For the latter, the difference is of substantial magnitude compared with other cases (0.79) and is statistically significant. The CSP proto-member also has high relevance in both mission and operations for providing food access to economically disadvantaged populations, regardless of racial ties. In contrast, for Coops both food access items rate low for relevance to both mission and operations, 
and three of the four of these differences are statistically significant for the species and of relatively high magnitude. It is interesting that the Processors' proto-member also rates food access for marginalized and disadvantaged populations relatively highly. However, overall addressing racial disparities is clearly less relevant for US FHOs' missions and daily operations, with the lowest total average of any of the 11 items queried of responding managers being for expanding food access for racial minorities followed by the second lowest being for expanding market access for minority-owned suppliers.

Average Joes and TPWs exhibit high relevance for improving human health, for promoting environmentally sustainable production practices, and for ensuring animal welfare. For the first species, these positive differences over others are statistically significant in relation to the FHO's mission, as well as for environmental production in relation to daily operations. This finding tracks with the large gap between Average Joes' average rating for these three items (in relation to mission) and the next highest species. This highlights an intriguing detail in the results that shows that although general trends between mission and operations ratings maintain, at individual species levels organizations may diverge in what they say they want to do through their mission and what their managers perceive they do in their daily operations. This may be a performative element driven by the psychology of scales like this (that is, where respondents feel a need to express that they meet social ideals in accounts of actual behaviors), but it may also legitimately indicate that FHOs do more in social values in practice than what they may state in their planned missions.

\subsubsection{Performance}

I examined firm performance through the lenses of total reported value of product moved through the FHO, revenue, and profit (Figure 5.14 presents mean profiles). It is 
unambiguous that three of the six species-TPWs, Coops, and Processors-have protomembers with substantially greater economic effect in total value of product moved and revenue. The other three species-Average Joes, CSPs, and Small-scale Startups—are within a 1-million-dollar ceiling and show little variation. The three top performers diverge in their mean profiles. Coops are the most profitable largely because of low expenses. It is interesting that only Average Joes show statistically significant mean differences for the core performance variables of total value of product moved and profit (see Table 5.2); the proto-member for the species earns 2.78 million dollars less in profit than all other cases as a group. This statistical significance is likely driven by the comparison being against a group of predominantly Processors, Coops, and TPWs. Because univariate examination ${ }^{79}$ of data has limitations, analysis is extended with regression estimation, accounting for factors exogenous to species effects. Table 5.4 shows the models and attendant results. In line with the original working paper, region and strategic orientation are factors. Manager age — originally used as a proxy for experience - is replaced with the estimated maximum number of total suppliers. Overall, model fit, measured by the adjusted $R^{2}$ value, is high for value of product moved but indicates that models for profit can likely improve in their explanatory power.

Results are consistent with the working paper in that (a) the only species showing a statistically significant coefficient is Coops; (b) only region 3 has a statistically significant environmental effect; (c) offering more types of community services has a positive and significant effect on profit; and (d) relying greatly on meat and poultry has a statistically significant and positive effect, whereas a greater share of sales from eggs has

\footnotetext{
${ }^{79}$ Mean profiles, analysis of variance, and independent-samples $t$ tests.
} 
a negative effect. Here, the scale proxy, in the form presented, is significant for value of product moved only. Coefficient magnitudes should be cautiously interpreted, but overall the region 3 effect on FHO performance is exceptionally large-between 10 and 19 million dollars for total value of product moved and 14 and 18 million dollars for profitability; the Coops species effect is smaller but still substantial—between roughly 7 and 9 million dollars for both performance measures. A strict interpretation of the meat and poultry effect (which should be taken cautiously) implies that for every 1 percent increase in reliance on the product category, there is a $\$ 110,000$ increase in total value of product moved and a $\$ 140,000$ gain in profit. These interpretations are ceteris paribus, which is a crucial assumption in understanding the results; recall that both Coops and meat and poultry are significant but also that Coops tend to more greatly rely on meat and poultry sales. When an interaction effect between species and meat and poultry reliance is included, both items and the interaction effect are statistically nonsignificant in the model.

\subsection{Discussion}

Taking an organizational species approach to FHOs does something unique: while the species are formed on a morphological basis, there are clearly distinct profilesproperty clusters - for each that occur within variables external to and separate from those used to form the groupings. This is not only for the strategic niches used to validate the final postulated cluster solution as a set of species, but also for other properties, such as those examined in this chapter that are also important to public policy decisions. Thus, this chapter provides corroborating support for the distinctness of these groups, at least at the snapshot in time that is the data in the 2015 National Food Hub Survey. It is important to note that these different profiles indicate that different species of FHOs may 
play distinct roles in food distribution systems, coordinating actors at different levels, with different backgrounds, in different ways, and with different effects.

One intriguing finding in the results presented here: one of those species, Coops, consistently sat below the population averages for most variables examined and yet was the only species indicated, with statistical significance within the range of accepted certainty (10 percent), as having not only a high effect on scaling up LRFSs through the total value of product moved through the coordinating intermediary but also being highly profitable. This is especially poignant when considering the nature of a cooperative juridical form of organization, in which ownership and control are one and the same, and thus these higher profits accrue directly to the member-user-owners of the FHO. From the AOTM approach, which seeks to scale up the primary agricultural producer firms within LRFSs, this is a crucial finding. Promoting and supporting cooperative juridical forms for FHOs — especially in foodsheds where an AOTM paradigm is prioritized—shows promise for achieving scalability. Moreover, as was found in Chapter 4 through an investigation of strategic profiles, these Coops FHOs expand the scope of products sold through intermediated channels, addressing market failures for small and medium abattoirs.

Other results indicate potential efficiency or financial concerns for the species with the largest overall scale - in number of suppliers in the network and total value of product moved — what I have named "traditional produce warehousers" (TPWs). It is difficult to say this confidently because neither the mean differences in independentsamples $t$ test analysis nor the species' coefficient in regression analysis, when accounting for exogenous variables, show statistical significance. I also note that the 
models for profit have low goodness-of-fit measures, indicating in part that other key variables omitted might help explain the variation in profitability. However, the observed difference in magnitudes for the sample still highlights the gulf between profitability and economic activity for these behemoths. If my theory about the origins of the TPW species - that TPWs as a species come from migration of firms into LRFS from conventional marketing channels - is eventually supported, additional investigation of evolutionary processes and mechanisms among organizational populations is warranted. If my theory were supported with additional empirical evidence, it would indicate that mimicry in local foods econosystems is not an effective strategy, no matter the effects on the overall system scale. Such a finding would support early concerns about authenticity and legitimation within the LRFM.

Results here also provide evidence that in making decisions on organizational form, trade-offs may be made, as indicated by the theory of a hybrid objective function, between profit maximizing behavior and the production of social welfare goods and services. Organizational species of larger scale had the lowest numbers of community service provision, inclusion of underrepresented minorities, relevance of social values to mission and operations, and - in some cases - more extended transactional arrangements. Of note too is that the majority of FHOs - contained in the species of Average Joes, Small-Scale Producers, and Community Service Providers - are still operating at small scales. These species present profiles of proto-members with more engagement in the costly provision of social goods but their functional scale capacities for aggregation, distribution, and processing of food products are limited. Moreover, across all species of FHOs, there still is not a singular organizational form that has broken off into a radically 
divergent product and/or channel strategy, with fresh produce sold via direct-to-consumer channels still predominant. FHOs have increased scale through channel choice by turning toward retail and restaurant markets; none of the species prioritize intermediated or institutional markets.

\subsection{Policy Implications and Conclusions}

From the perspective of policymakers, these results provide guidance at two main levels: (1) overall investments that can be made in FHOs to promote scaling up of LRFSs and (2) targeted policy choices to address local needs of a particular managed foodshed. To address item 1, different strategies may be adopted. For some communities, increasing the scale of the entire system may be achieved through transitioning a large for-profit warehouse to a source-identified purveyor or by making large capital investments to increase the capacity of an existing Small-scale Startup. This transition may be funded by the firm's operations or from private sources such as foundation grants and individual donors. In other scenarios, a spoke-and-wheel approach, where support is provided to form a network of Average Joe FHOs that meet local needs but have a supra-collective coordinating among system FHOs, may be best suited. Practitioners and policymakers increasingly discuss such models. Where goals include not only total system scale but also the scale of farms and ranches supplying the FHO, stimulating the formation of a cooperative-where incentives are aligned so that growing the FHO supports growth among member-owners - is the best strategy. Findings here show that the direction chosen by policymakers will have different implications for the community services and social values likely to be pursued by the FHO, as well as the scope and nature of products traveling through the intermediated marketing channel. This means that decisions about 
stimulating a specific species population to achieve overall improvements of LRFS scale must account for these trade-offs.

Considering item 2, results here also help guide foodshed decision makers faced with specific, idiosyncratic goals, such as to fill particular gaps while also increasing scale. One such example is for foodsheds, where the goal includes to increase not only scale but also value-added products. Small-scale Startups are less than ideal, and policymakers should make critical investments in training and infrastructure that can move such organizations toward processing. These interventions should target not only knowledge and infrastructure narrowly within the processing function but also building organizational capacity to form and manage supply networks with other distributors, managerial capabilities that shift sourcing of processed products from external partners to the FHO itself (that is, vertical integration), and compliance with food safety regulations. On the basis of the results in Figure 5.7, funding for these investments will likely come from state and local government sources.

In communities that face racial and economic disparities, CSP-like organizations with a greater emphasis on social work functions may be more practicable. Implications of results here, triangulated with qualitative research on background, are that these organizations form hyperlocal micro-networks among producers—-such as urban agriculturalists—and consumers in close proximity. Typically nonprofits, these organizations are extensions of public or civil society initiatives, and thus investment in public-private partnerships, civic granting programs, and other public policies (such as property tax abatement for revitalization) may be effective in stimulating CSP populations locally. These organizations are likely to provide avenues for small and 
medium-sized enterprises in the processing sector as much as - if not more than-those in primary production - likely mom-and-pop prepared foods with value addition, based on qualitative observations - as well as for nonfood businesses. Creating an enabling environment hospitable to CSP FHOs may also stimulate new venture creation, an attractive feature in areas where startup incubation is a central goal. Policy orientations that promote CSPs have implications for the scope of the LRFS, whereas overall increases to scale may be generally minor, with most scale-enhancing effects on relative growth for low-baseline foodsheds and through gains in equity of economic participation.

In contrast, for portions of foodshed in relatively wealthy areas-especially those with vibrant specialty restaurant sectors-support for new foundings of midscale (Average Joes) and small-scale (Small-scale Startups) FHOs can be ideal. This approach seeks to address the scaling-up challenge using FHOs by increasing the total number of coordinating intermediaries in the foodshed rather than increasing the capacity of individual firms. Under this strategy, a greater number of intermediaries engaged in planning, aggregation, assortment, and distribution to technically advanced buyers (restauranteurs) that require consistency and variety stimulates value creation and capture. The majority of products moving through channels under this strategy are specialty crops (fresh and processed produce), with value largely driven by nontangible product attributes such as localness, novelty, and sustainable production practices, not through advanced processing. For Small-scale Startups especially, investments in training programs for managers on the core functions of FHO operations-including warehousing logistics for foodstuffs, management, strategic planning, and sales and marketing—are crucial for success. Funding for establishing and expanding FHOs under this approach 
may come from a variety of sources, thus investing in financial coordination and support is necessary, especially because it is expected that most sourcing will come from a collection of private, small-scale transactions such as membership fees, private donations, and business loans.

For foodsheds that want to scale up primarily through scope (perhaps in regions where marketing infrastructure of LRFSs for specialty crops is already well-developed), co-ops — and, to a lesser extent, TPWs — should be considered. It is notable that enabling environments for these species are also appropriate targets when the objective for scope is not only products but also upstream marketing channels, such as expanding provision of local foods to schools, hospitals, and other intermediaries. Promoting these species of FHOs may negatively affect certain community and social attributes, and thus they may not be well suited for situations where social capital investments are crucially needed. Coops especially could benefit from investments in holistic manager training programs, perhaps intensive courses that promote broad-spectrum skills development. Meanwhile, TPWs could most benefit from narrowly targeted programming that improves knowledge about agricultural production practices. This is expected to be particularly important given new food safety regulations under the Food Safety Modernization Act, where FHO managers knowledgeable of on-farm production can empower them to make more appropriate decisions about sourcing and the effect of certification programs on growers. The choice between the two strategies critically depends on having funds available from public and nonprofit sources in the environment (TPWs greatly rely on these, whereas Coops use private equity) and consumer and agroclimatic factors (from my data, Coops 
are especially useful in places with demand and growing conditions conducive to meat and dairy).

These few selected examples highlight the importance of approaching econosystems ecologically; different organizational forms cannot be expected to play the same role in the networks of economic agents that comprise the SFSCs that make up LRFSs. In developing policy interventions to actively manage any given foodshed landscape, we must account for the roles of these different organizational forms. They engage in economic activity differently, creating different connections and incorporating different kinds agents, and thus have different effects on the communities in which they are based and different overall performance. The evidence I present in this and the previous chapter indicates that a species approach to organizational form-in which kinds are developed on the basis of observed morphological features of cases and boundaries between forms validated by showing that they act within a specific nichecan be used to identify specific policy interventions that meet local objectives and idiosyncratic environmental conditions. Whereas decision makers may adopt policies to promote establishing or expanding FHOs in the foodsheds they manage, mal-selection of policy instruments may not stimulate growth of the population with an organizational form best suited to the prevailing environmental conditions or that meets the underlying objectives. Such mal-selection may even inadvertently stimulate a population that does not adequately fill the targeted niche. This makes environmental assessment, clear delineation of policy objectives on the basis of that assessment, and appropriate governance mechanisms critical antecedents of an ecological approach to managing LRFSs. 


\subsection{Tables and Figures}

Table 5.1 Mean Differences in Supplier and Manager Experience Variables: Species Versus All Other Observations

\begin{tabular}{|c|c|c|c|c|c|c|}
\hline Variable & $\begin{array}{c}\text { Average } \\
\text { Joes }\end{array}$ & $\begin{array}{c}\text { Small-Scale } \\
\text { Startups } \\
\end{array}$ & Processors & $\begin{array}{c}\text { Community } \\
\text { Service Providers }\end{array}$ & Coops & \begin{tabular}{|c|}
$\begin{array}{c}\text { Traditional Produce } \\
\text { Warehousers }\end{array}$ \\
\end{tabular} \\
\hline Farms or ranches not owned or managed by the food hub & -0.07 & -0.01 & -0.04 & 0.04 & 0.04 & -0.10 \\
\hline Food processors not owned or managed by the food hub & 0.00 & -0.16 & 0.02 & -0.29 & 0.12 & -0.16 \\
\hline A different food distributor & -0.06 & 0.26 & -0.04 & 0.07 & 0.15 & -0.21 \\
\hline The food hub's own farms, ranches, and other enterprises & 0.00 & 0.28 & -0.19 & 0.00 & 0.00 & 0.00 \\
\hline Non-food-related businesses & 0.06 & 0.08 & -0.03 & -0.24 & -0.04 & -0.02 \\
\hline Other (specify) & 0.08 & 0.07 & 0.01 & -0.07 & 0.00 & 0.07 \\
\hline Total number of supplying enterprises (all types) & 25.86 & 57.83 & -25.91 & 60.05 & 44.38 & -210.21 \\
\hline Number of supplying enterprises (farms or ranches only) & -1.40 & 15.38 & -0.86 & 22.31 & -1.31 & -41.93 \\
\hline Estimated maximum number of suppliers & 26.38 & 46.33 & -24.58 & 48.26 & 27.86 & -160.61 \\
\hline Percentage of suppliers who are farms or ranches & -1.83 & -4.15 & -1.52 & -8.07 & 3.78 & 8.29 \\
\hline Firms owned and operated by women (\% of suppliers) & 6.38 & -7.46 & -7.85 & 2.68 & 0.41 & 4.21 \\
\hline Firms owned and operated by people of color (\% of suppliers) & 6.63 & -2.07 & -14.62 & -35.29 & 17.67 & 7.47 \\
\hline Firms in operation for fewer than 10 years ( $\%$ of suppliers) & 4.05 & -5.41 & 20.84 & -11.97 & -2.72 & -0.56 \\
\hline Age of senior manager & -2.94 & 3.33 & 1.01 & -6.47 & 0.81 & -0.22 \\
\hline Education level of senior manager & 0.21 & -0.34 & -0.42 & 0.73 & 0.50 & -0.46 \\
\hline Warehousing and distribution of food & -0.70 & 0.94 & 0.07 & -0.41 & 0.36 & -0.85 \\
\hline Management, including financial and capital planning & -0.77 & 1.15 & -0.42 & -0.52 & 0.68 & -0.49 \\
\hline Strategic planning & -0.71 & 0.44 & -0.42 & 0.29 & 0.59 & -0.75 \\
\hline Food processing & 0.25 & 0.11 & -0.74 & -0.18 & 0.58 & -0.31 \\
\hline Food marketing and sales & -0.26 & 0.44 & 0.38 & -0.41 & 0.36 & -0.39 \\
\hline Food retail & -0.09 & 0.03 & 0.87 & -0.63 & 0.65 & -0.12 \\
\hline Agricultural production & -0.01 & -0.32 & -1.27 & -0.84 & 0.50 & 0.87 \\
\hline Using other food hub-related skills & -0.33 & 0.20 & -0.74 & 0.20 & 1.22 & -1.31 \\
\hline
\end{tabular}

Source: Author's calculations.

Note: Values in bold indicate statistical significance at or beyond the 10 percent level. 
Table 5.2 Mean Differences in Financial and Performance Variables: Species Versus All Other Observations

\begin{tabular}{|c|c|c|c|c|c|c|}
\hline Variable & $\begin{array}{l}\text { Average } \\
\text { Joes }\end{array}$ & $\begin{array}{c}\text { Small- } \\
\text { Scale } \\
\text { Startups }\end{array}$ & Processors & CSPs & Coops & TPWs \\
\hline $\begin{array}{l}\text { Income from other programs of the } \\
\text { organization }\end{array}$ & 0.05 & -0.11 & -0.18 & 0.19 & 0.20 & -0.51 \\
\hline Business loans & -0.13 & 0.01 & -0.08 & 0.10 & 0.11 & 0.10 \\
\hline Federal government funding & 0.18 & 0.06 & -0.12 & -0.09 & 0.08 & -0.01 \\
\hline State government funding & 0.06 & 0.17 & -0.22 & 0.04 & -0.02 & -0.10 \\
\hline Local government funding & -0.01 & 0.00 & 0.02 & 0.19 & -0.08 & -0.16 \\
\hline Foundation grants & 0.02 & -0.02 & 0.02 & 0.16 & -0.03 & -0.68 \\
\hline In-kind support & 0.00 & 0.07 & 0.10 & -0.07 & -0.05 & 0.00 \\
\hline Donations from organizations & 0.01 & -0.14 & -0.02 & 0.16 & 0.03 & 0.16 \\
\hline Donations from individuals & 0.15 & -0.13 & 0.00 & -0.16 & 0.00 & $-\mathbf{0 . 4 3}$ \\
\hline $\begin{array}{l}\text { Infrastructure provided by a } \\
\text { government entity }\end{array}$ & -0.04 & 0.08 & 0.08 & -0.14 & -0.06 & 0.07 \\
\hline Membership fees & 0.16 & -0.18 & 0.15 & 0.01 & -0.47 & 0.22 \\
\hline Bank loans & -0.07 & 0.16 & -0.03 & -0.07 & -0.27 & 0.14 \\
\hline Private investors & -0.21 & 0.06 & -0.03 & 0.15 & 0.16 & 0.14 \\
\hline $\begin{array}{l}\text { Organization's capital, founder's own } \\
\text { capital, or both }\end{array}$ & -0.21 & 0.24 & -0.13 & -0.14 & 0.11 & -0.20 \\
\hline Other (specify) & 0.07 & 0.11 & 0.02 & 0.10 & -0.03 & 0.10 \\
\hline $\begin{array}{l}\text { Total number of startup funding } \\
\text { sources }\end{array}$ & 0.04 & 0.37 & -0.43 & 0.45 & -0.31 & -1.16 \\
\hline Grant dependency rating & 0.08 & -0.08 & -0.07 & 0.08 & 0.24 & -0.05 \\
\hline $\begin{array}{l}\text { Food and/or product purchases from } \\
\text { producers and suppliers }\end{array}$ & -0.03 & 0.04 & -0.03 & 0.22 & -0.01 & -0.06 \\
\hline Packaging equipment and supplies & -0.01 & 0.01 & -0.02 & 0.01 & 0.01 & 0.00 \\
\hline Payments toward facilities & -0.01 & -0.03 & 0.00 & -0.06 & 0.03 & 0.02 \\
\hline $\begin{array}{l}\text { Payments toward trucks or other } \\
\text { automotive equipment }\end{array}$ & -0.01 & 0.02 & -0.01 & -0.07 & 0.02 & 0.01 \\
\hline Gasoline and tolls & 0.00 & 0.01 & 0.00 & -0.03 & 0.00 & -0.01 \\
\hline Repair and maintenance & 0.00 & 0.01 & -0.01 & -0.03 & 0.00 & 0.01 \\
\hline Utilities & 0.00 & 0.01 & -0.01 & -0.06 & 0.01 & 0.00 \\
\hline Advertising and promotional materials & $\mathbf{0 . 0 1}$ & -0.02 & 0.01 & -0.01 & 0.00 & 0.00 \\
\hline Credit card and bank service charges & 0.02 & -0.03 & 0.02 & -0.01 & 0.01 & 0.01 \\
\hline Employee salary and benefits & 0.02 & 0.05 & -0.04 & -0.02 & -0.03 & -0.02 \\
\hline Other Administrative expenses & 0.01 & 0.01 & 0.01 & -0.01 & -0.02 & 0.01 \\
\hline Data and computer services & 0.01 & -0.03 & 0.01 & -0.01 & 0.00 & 0.01 \\
\hline All types of insurance & -0.01 & 0.01 & 0.01 & -0.02 & 0.01 & 0.01 \\
\hline Consulting services & 0.01 & -0.04 & 0.02 & 0.02 & -0.02 & 0.02 \\
\hline Telecommunications & 0.00 & 0.00 & 0.00 & 0.00 & 0.00 & 0.00 \\
\hline Other & -0.01 & -0.02 & 0.03 & 0.09 & 0.00 & -0.01 \\
\hline Total expenses (in hundred thousands) & 1.75 & 2.28 & -2.70 & 1.98 & 1.06 & -6.26 \\
\hline $\begin{array}{l}\text { Total value of product moved (in } \\
\text { hundred thousands) }\end{array}$ & 2.78 & 3.18 & -0.39 & 2.79 & -5.39 & -6.38 \\
\hline Profit (in hundred thousands) & 1.70 & 1.62 & 1.00 & 1.46 & -7.17 & 1.70 \\
\hline
\end{tabular}

Source: Author's calculations.

Note: Value in bold indicates statistical significance at or beyond the 10 percent level. CSP $=$ Community Service Providers; TPW $=$ Traditional Produce Warehousers. 
Figure 5.1 Mean Profiles of FHO Species: Types of Suppliers Used

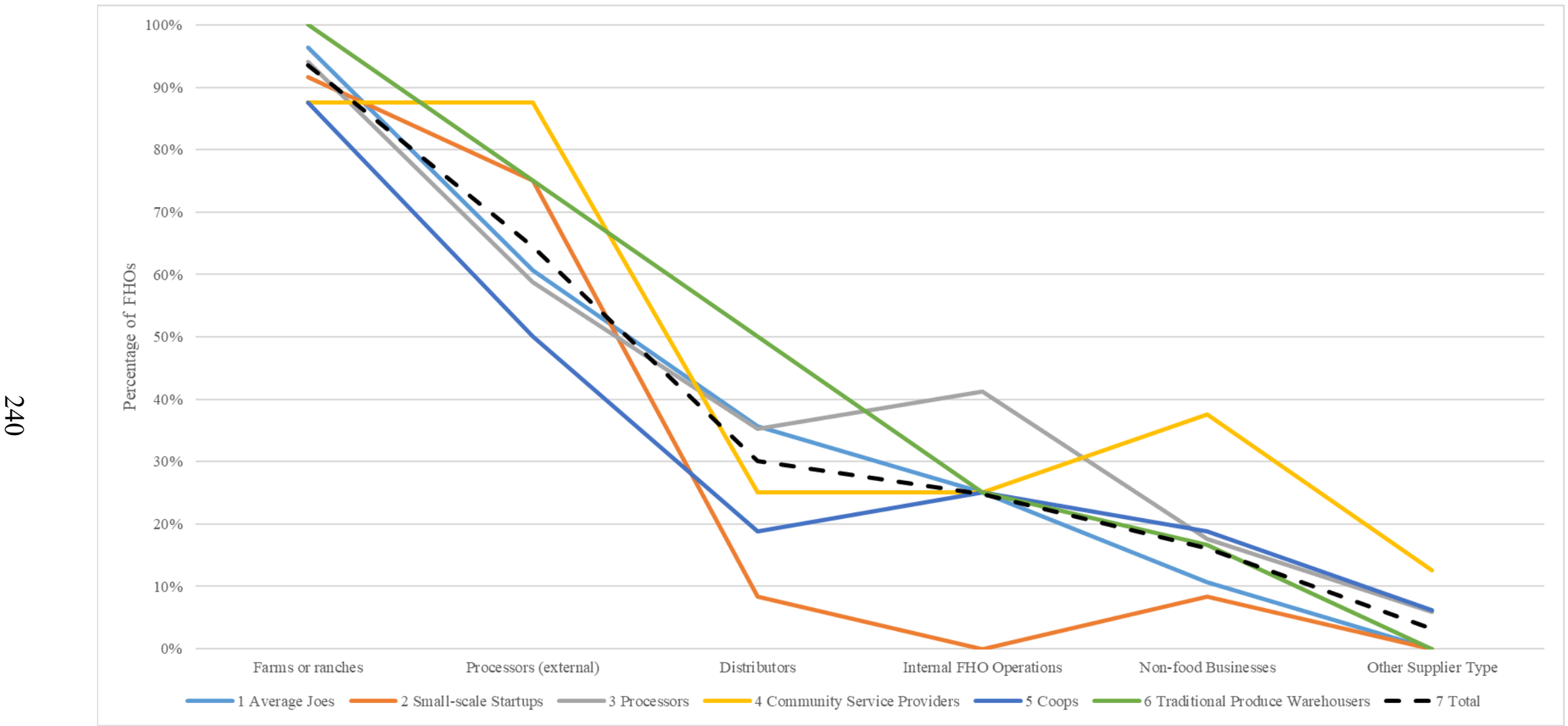

Source: Author

Note: $\mathrm{FHO}=$ food hub organization 
Figure 5.2 Types of Suppliers Used, by FHO Species

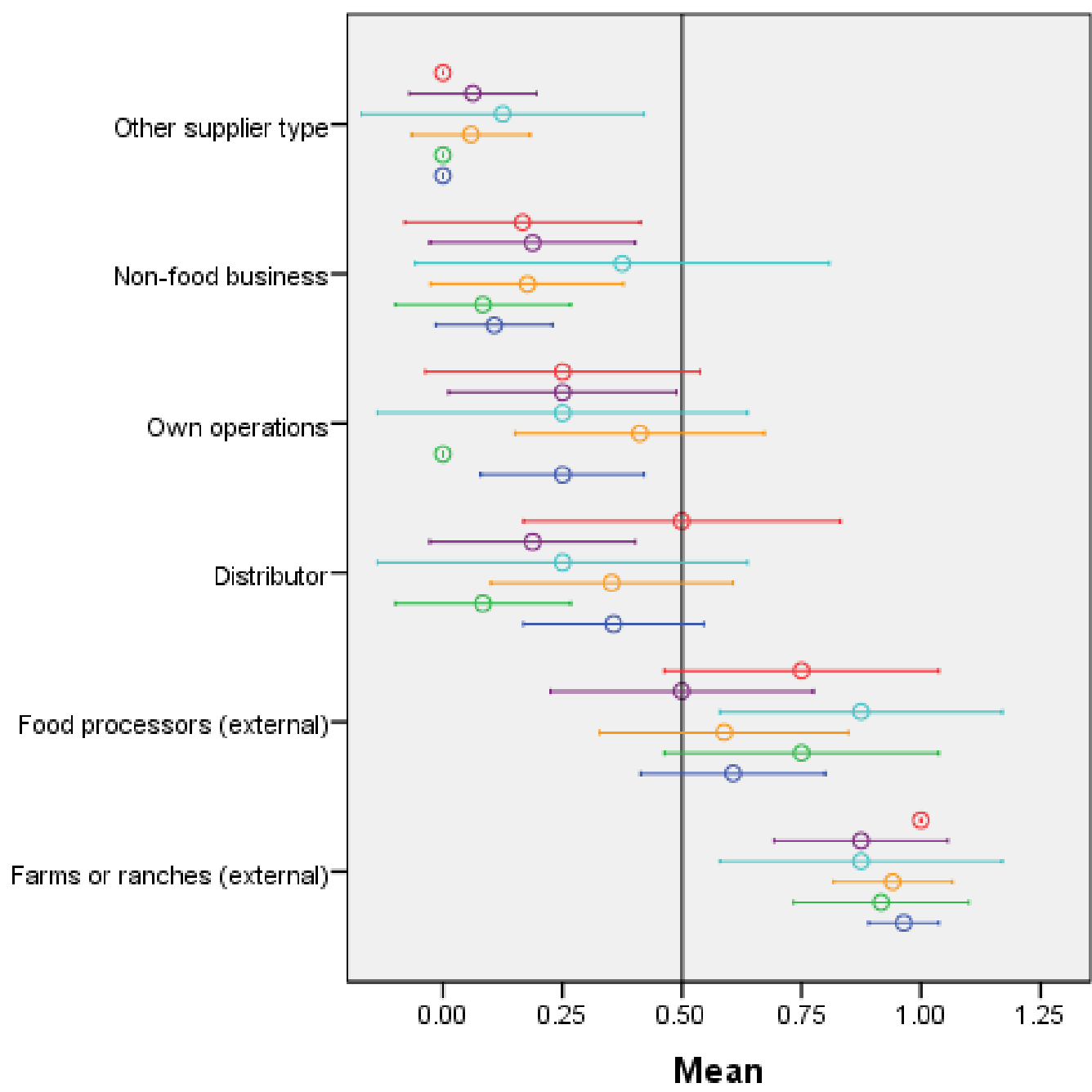

Error Bars: $95 \% \mathrm{Cl}$
Model $5, k=6$ **

$$
\text { [FPCS] }
$$

Average Joes

Small-scale Startups

Processors

Community Service

Providers

Coops

Traditional Produce

Warehousers

Note: $\mathrm{FHO}=$ food hub organization; FPCS $=$ final postulated clustering solution . 
Figure 5.3 Mean Profiles of FHO Species: Demographics of Suppliers

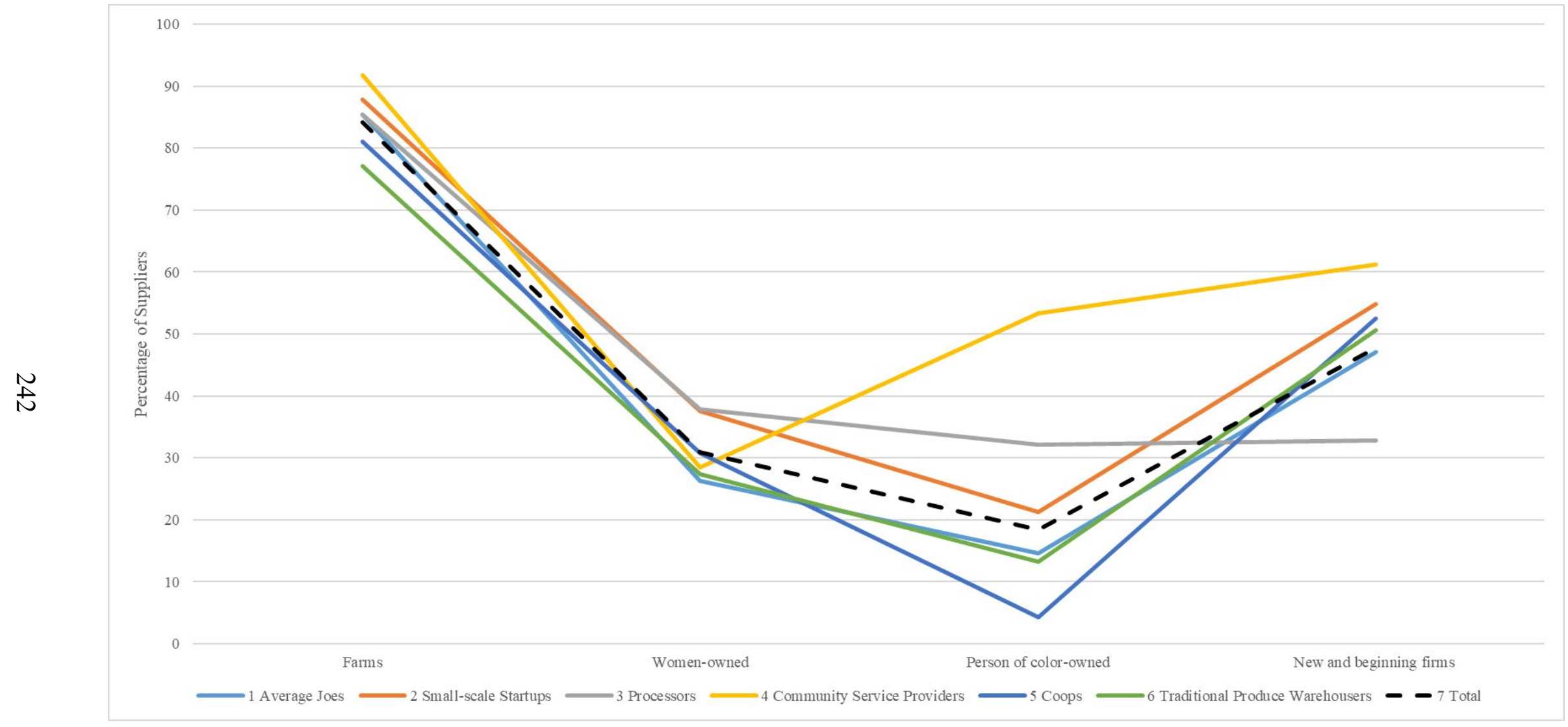

Source: Author.

Note: $\mathrm{FHO}=$ food hub organization 
Figure 5.4 Demographics of Suppliers, by FHO Species

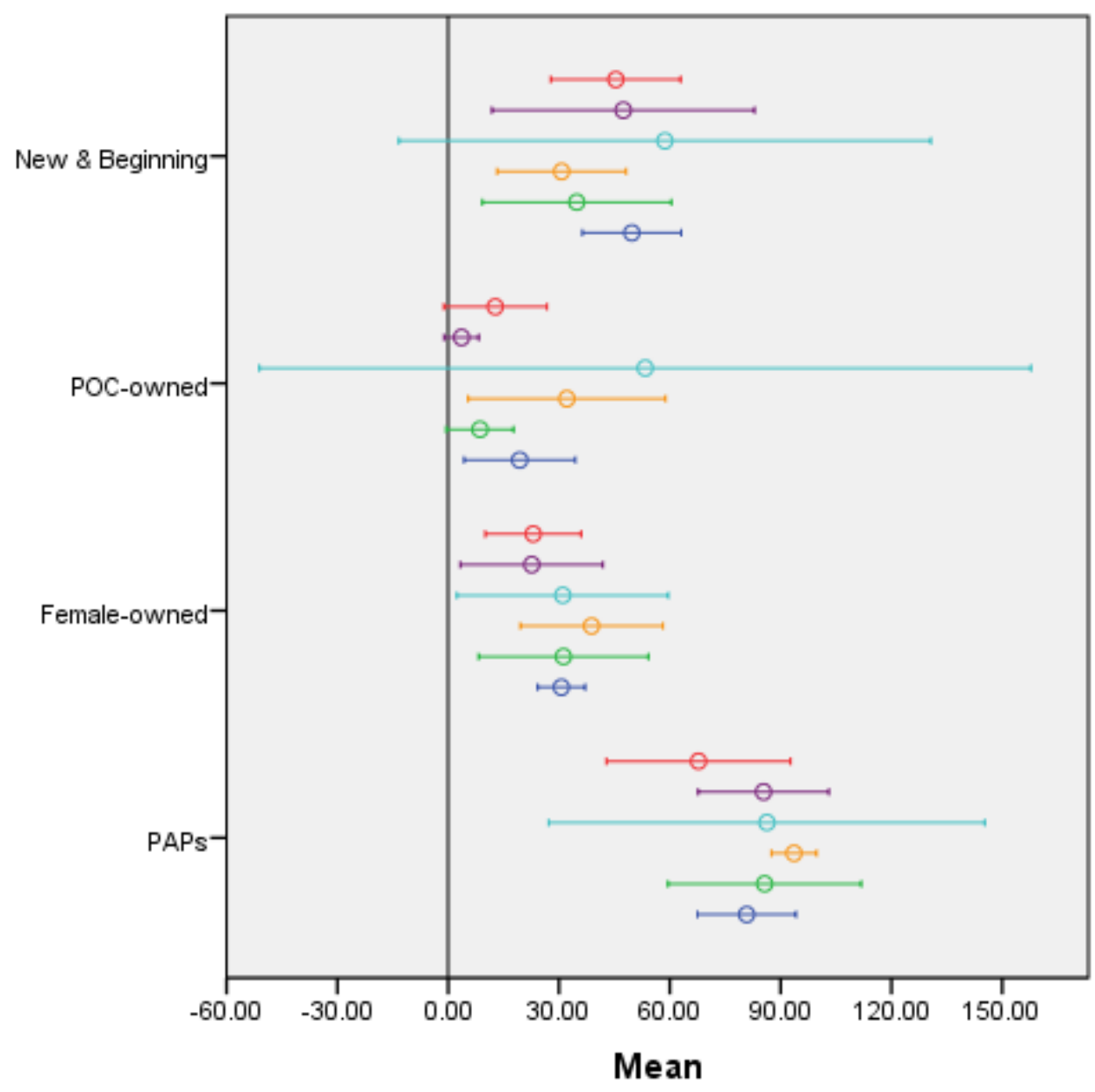

Model $5, k=6$ *ᄎ

$$
\text { [FPCS] }
$$

Average Joes

Small-scale Startups

Processors

Community Service

Providers

Coops

Traditional Produce

Warehousers

\section{Error Bars: $95 \% \mathrm{Cl}$}

Source: Author

Note: $\mathrm{FHO}=$ food hub organization; FPCS = final postulated clustering solution . 
Figure 5.5 Mean Profiles of FHO Species: Experience of Top Manager in Key Functions of FHOs

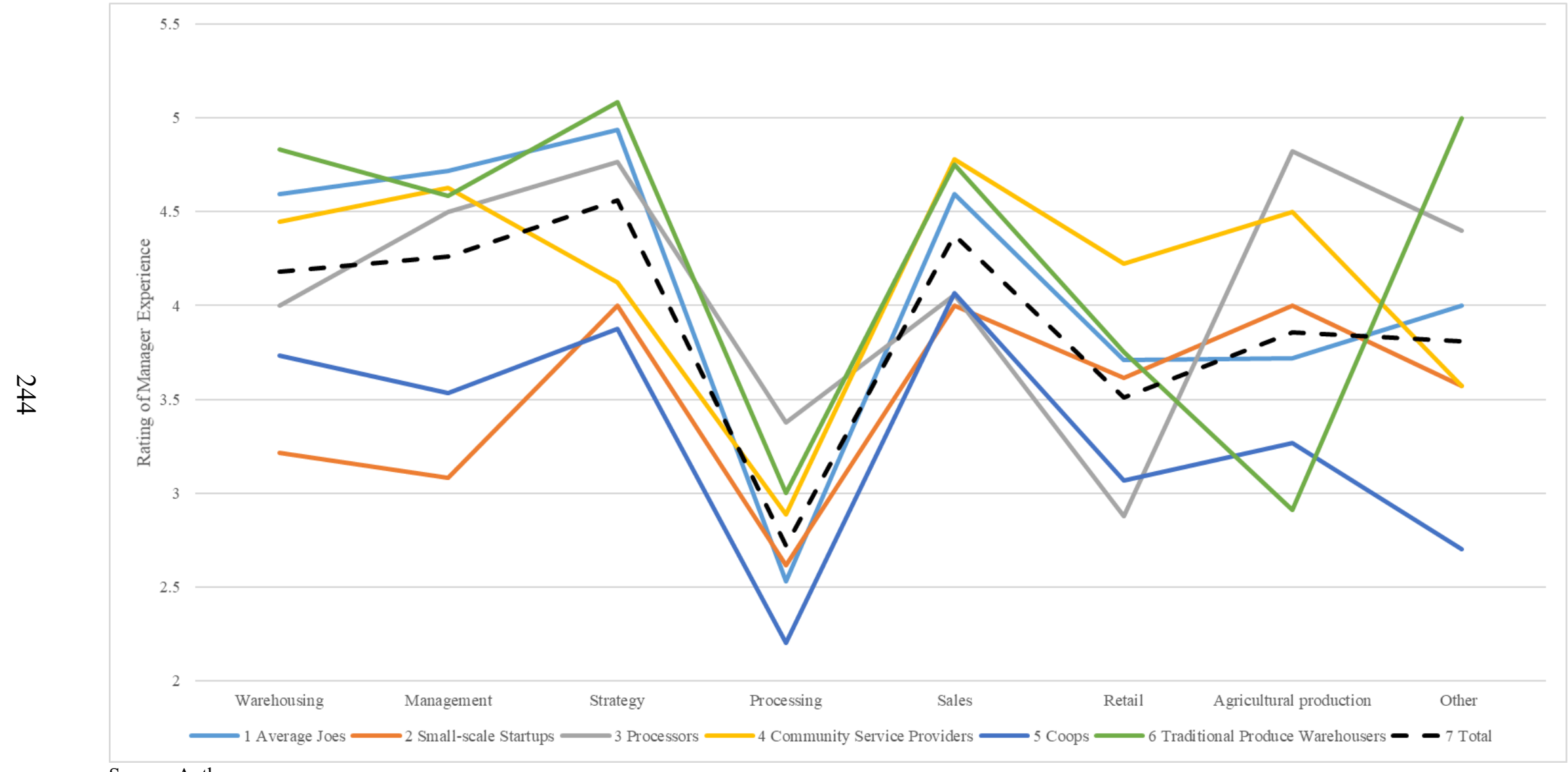

Source: Author.

Note: $\mathrm{FHO}=$ food hub organization. 
Figure 5.6 Manager Experience in Key Functions, by FHO Species

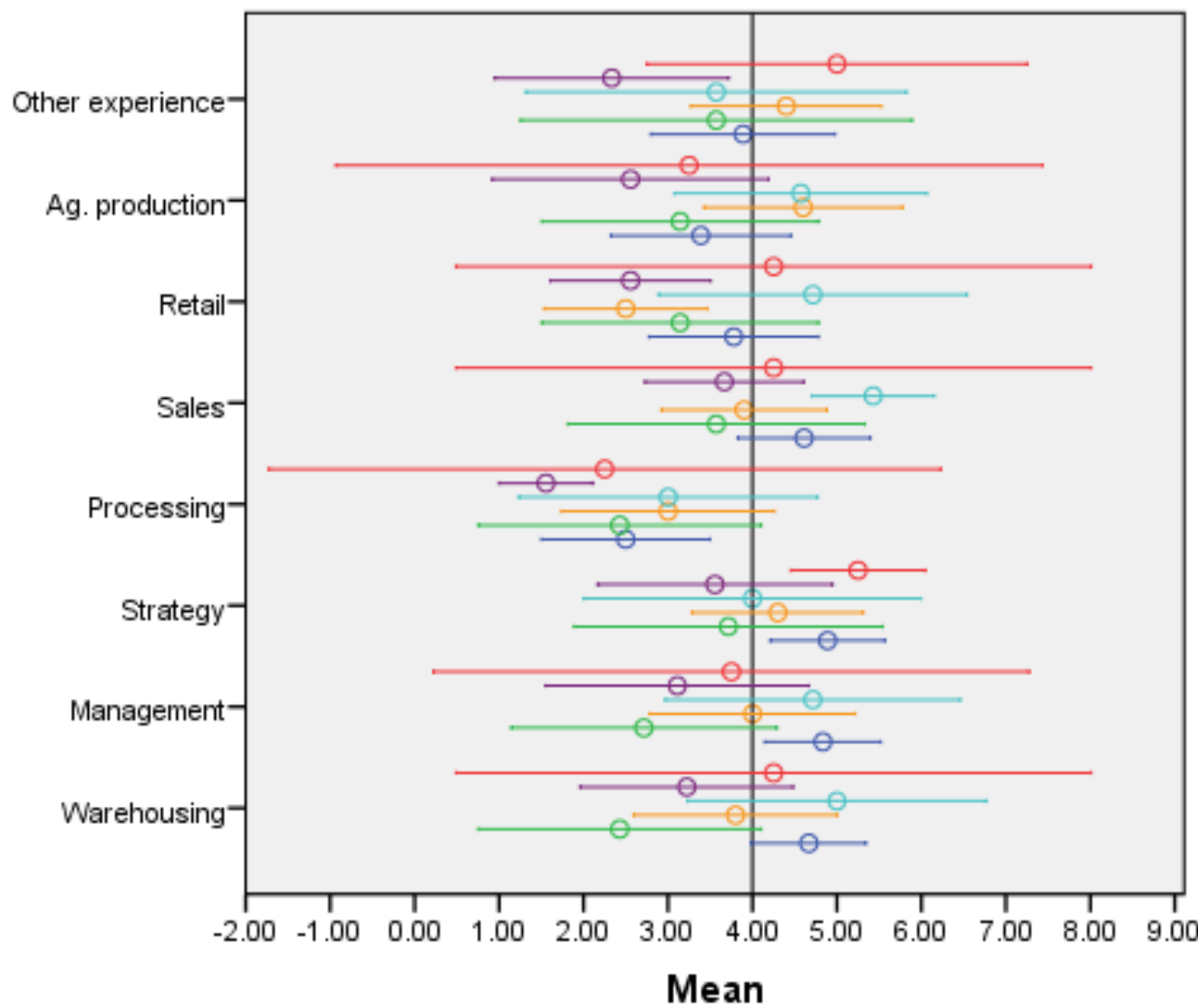

Model $5, k=6$ * [FPCS]

Average Joes

Small-scale Startups

Processors

Community Service

Providers

Ocoops

Traditional Produce

Warehousers

\section{Error Bars: $95 \% \mathrm{Cl}$}

Source: Author.

Note: $\mathrm{FHO}=$ food hub organization; FPCS $=$ final postulated clustering solution . 
Figure 5.7 Mean Profiles of FHO Species: Sources of Startup Funding

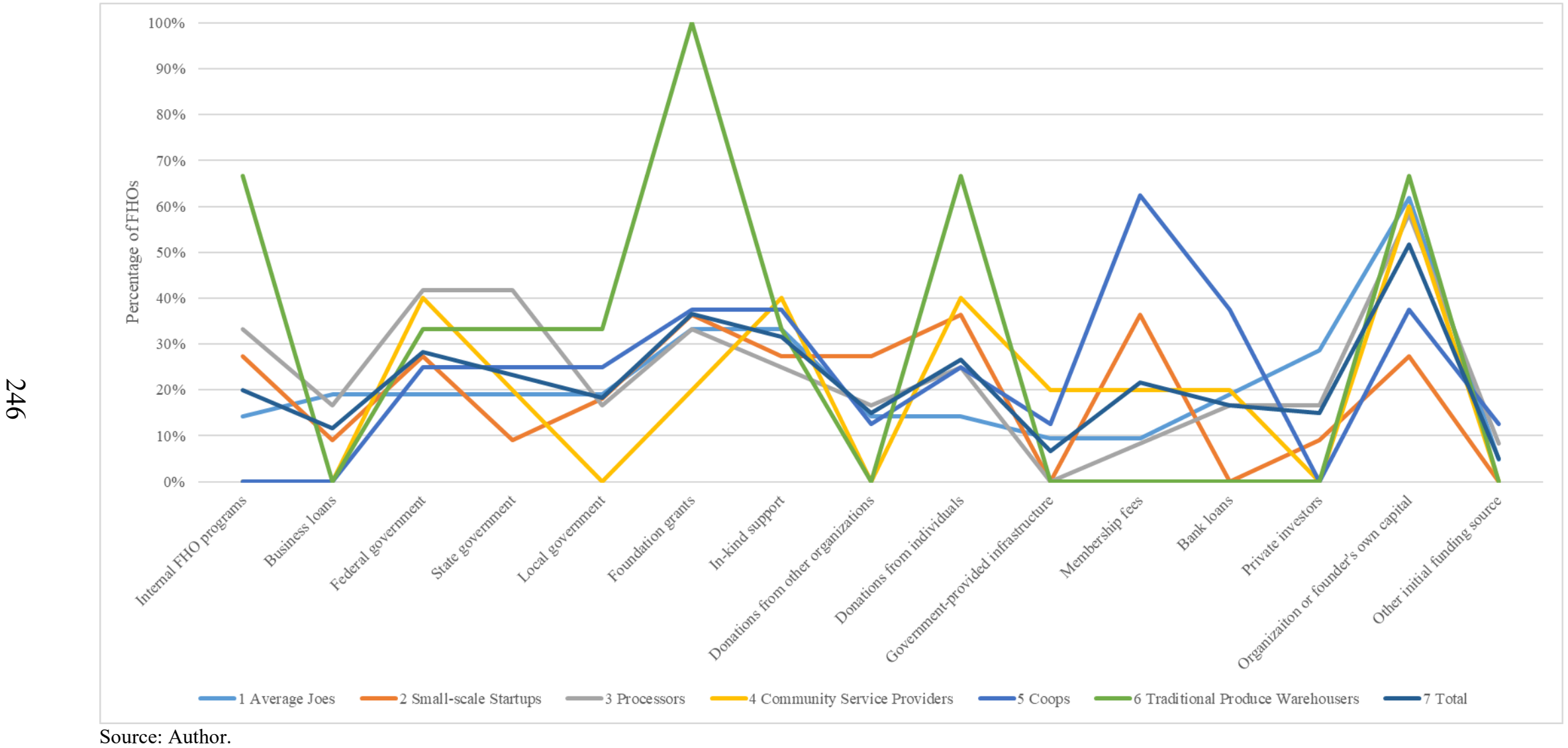

Source: Author.

Note: $\mathrm{FHO}=$ food hub organization. 
Figure 5.8 Sources of Startup Funding, by FHO Species

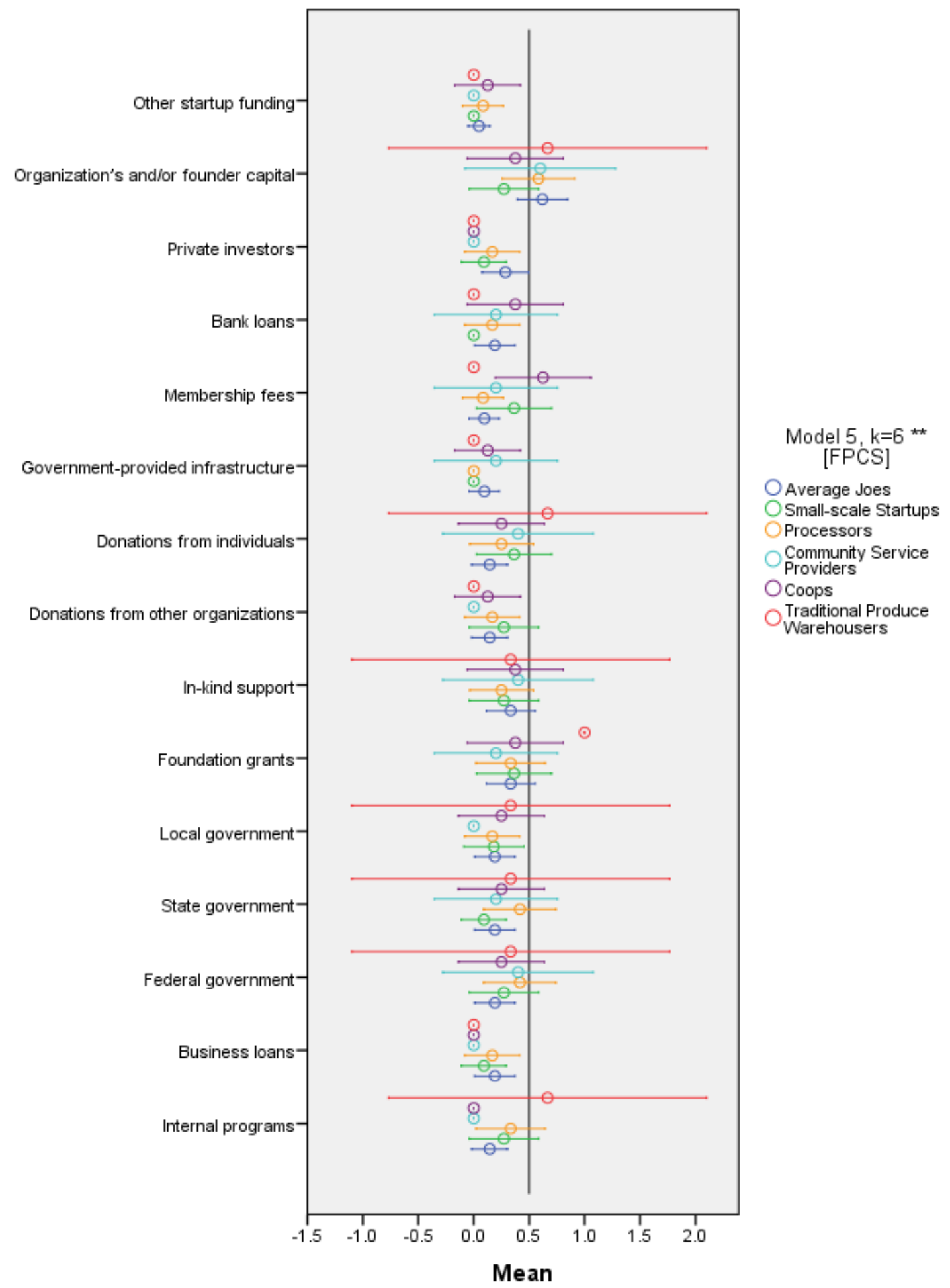

Error Bars: $95 \% \mathrm{Cl}$

Source: Author.

Note: $\mathrm{FHO}=$ food hub organization. FPCS $=$ final postulated clustering solution. 
Figure 5.9 Mean Profiles of FHO Species: Percentage of Total Costs for Each Category

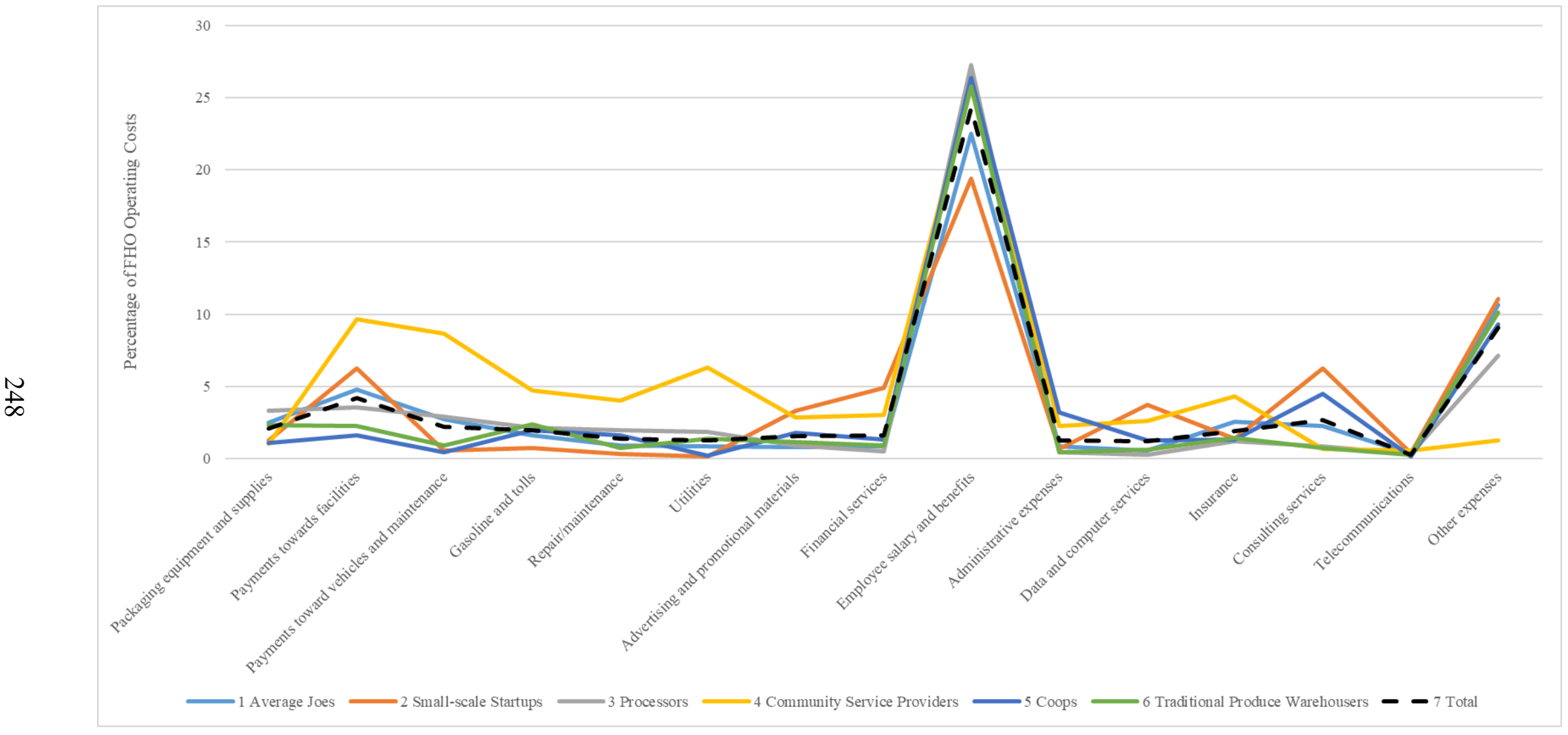

Source: Author.

Note: $\mathrm{FHO}=$ food hub organization 
Figure 5.10 Percentage of Total Costs for Each Category, by FHO Species

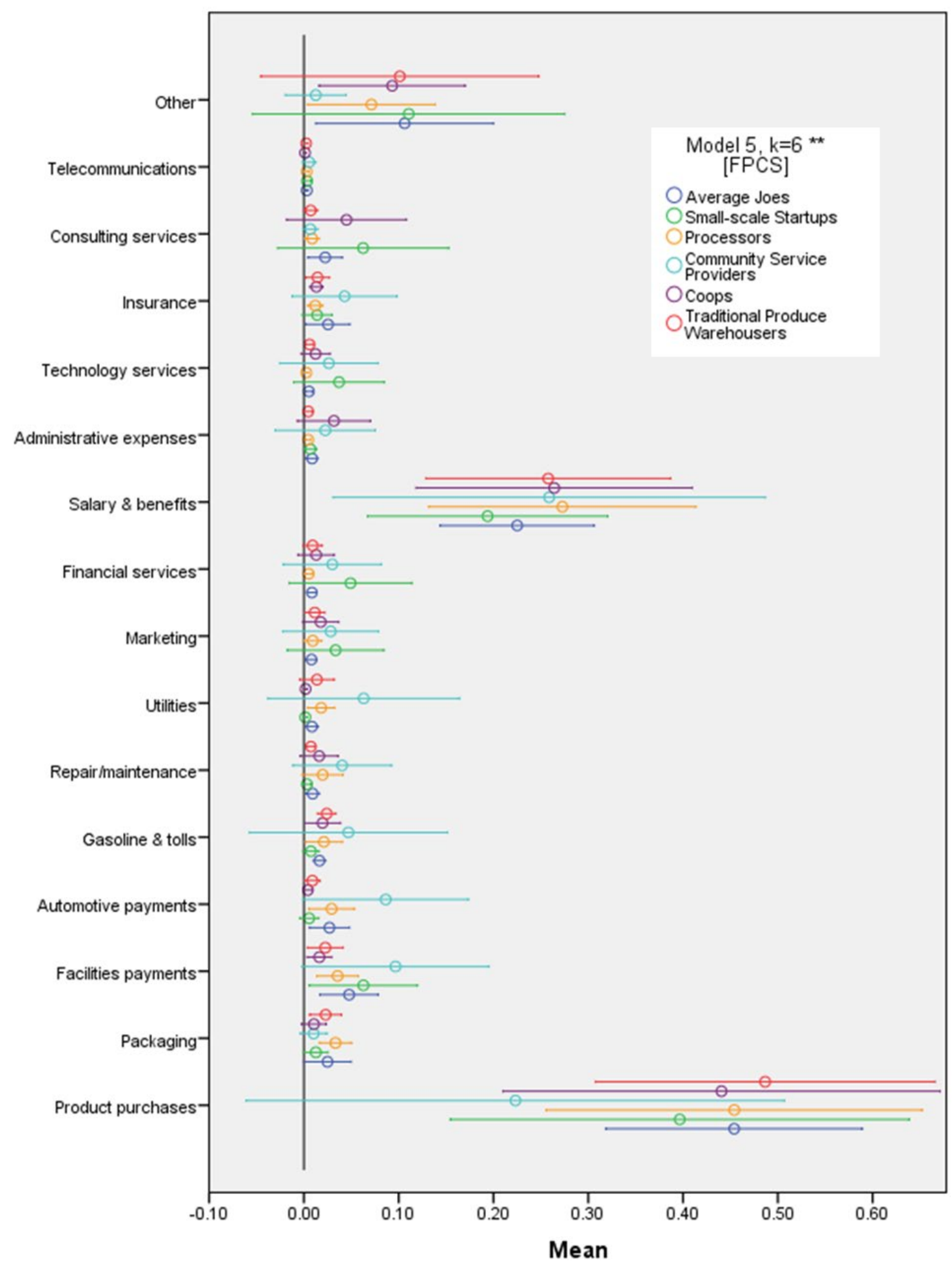

Error Bars: $95 \% \mathrm{Cl}$

Source: Author.

Note: $\mathrm{FHO}=$ food hub organization; FPCS = final postulated clustering solution. 
Figure 5.11 Mean Profiles of FHO Species: Relevance of Social Values to Organization Mission and Day-to-Day Operations

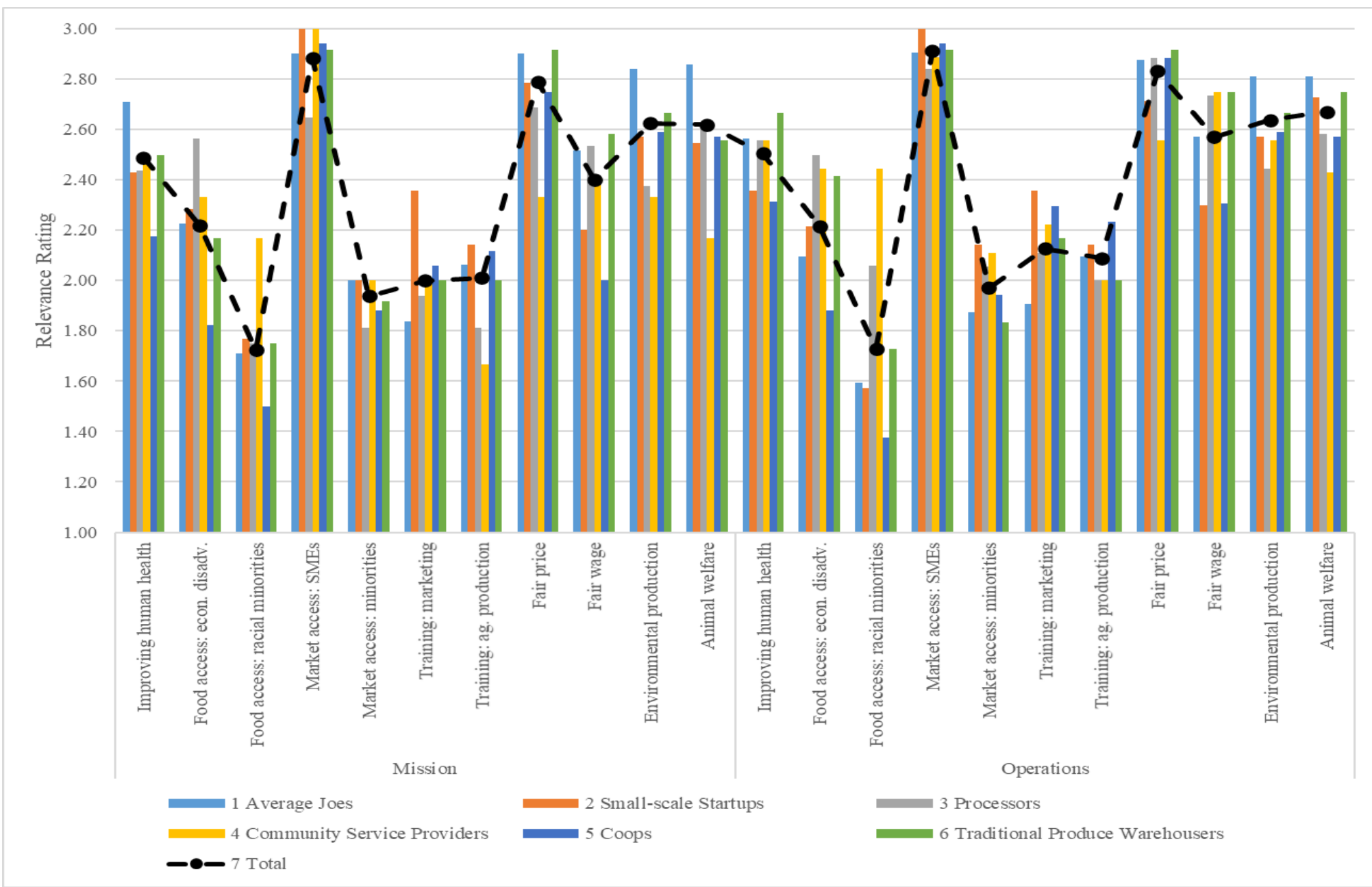

Source: Author

Note: Ag. = agricultural; Econ. disadv. = economically disadvantaged populations; FHO = food hub organization; $\mathrm{SME}=$ small- and medium enterprise. 
Figure 5.12 Relevance of Social Values to Organization Mission, by FHO Species

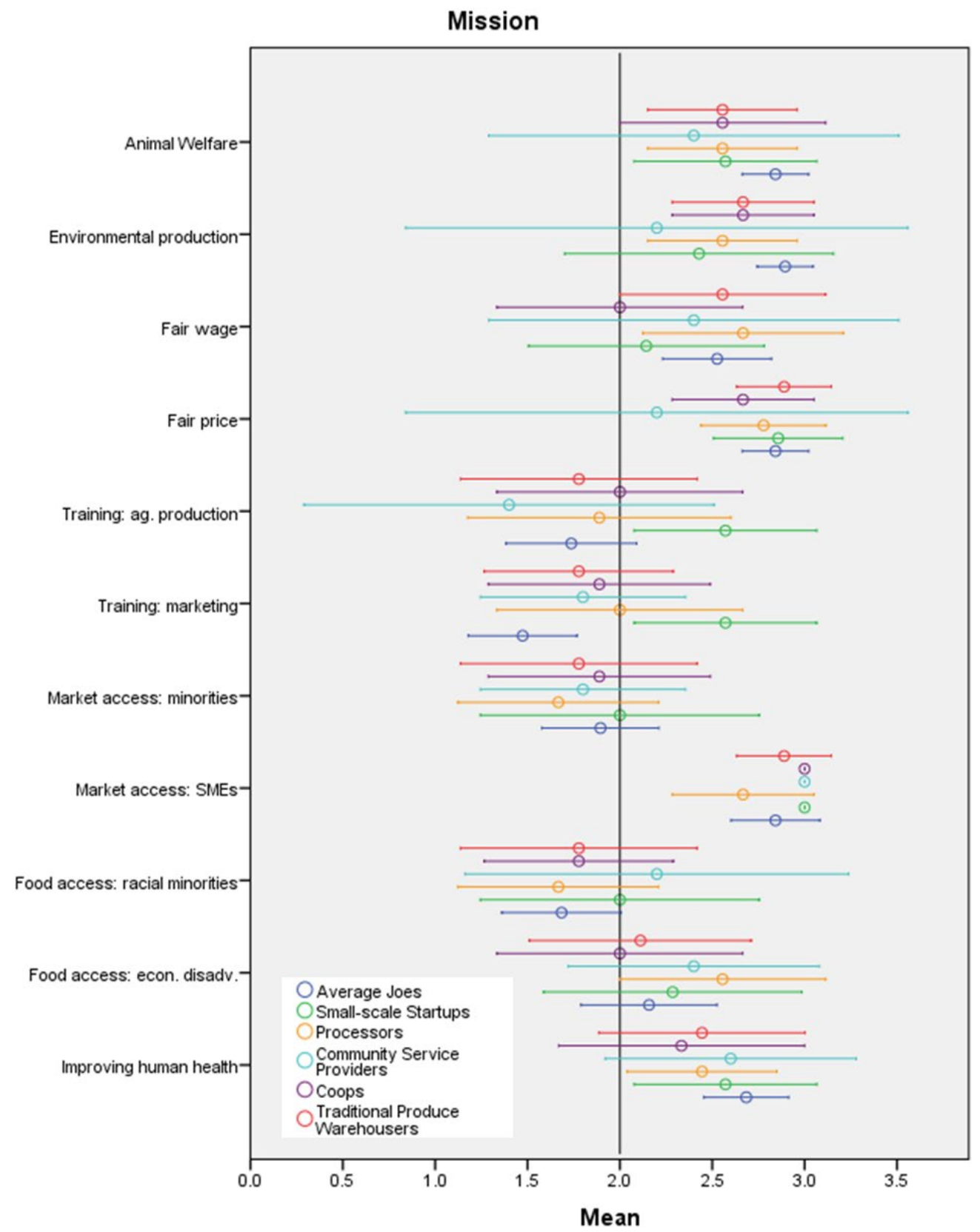

Error Bars: $95 \% \mathrm{Cl}$

Source: Author.

Note: Ag. = agricultural; Econ. disadv. = economically disadvantaged populations; $\mathrm{FHO}=$ food hub organization; SME $=$ small- and medium enterprise. 
Figure 5.13 Relevance of Social Values to Organizations' Day-to-Day Operations, by FHO Species

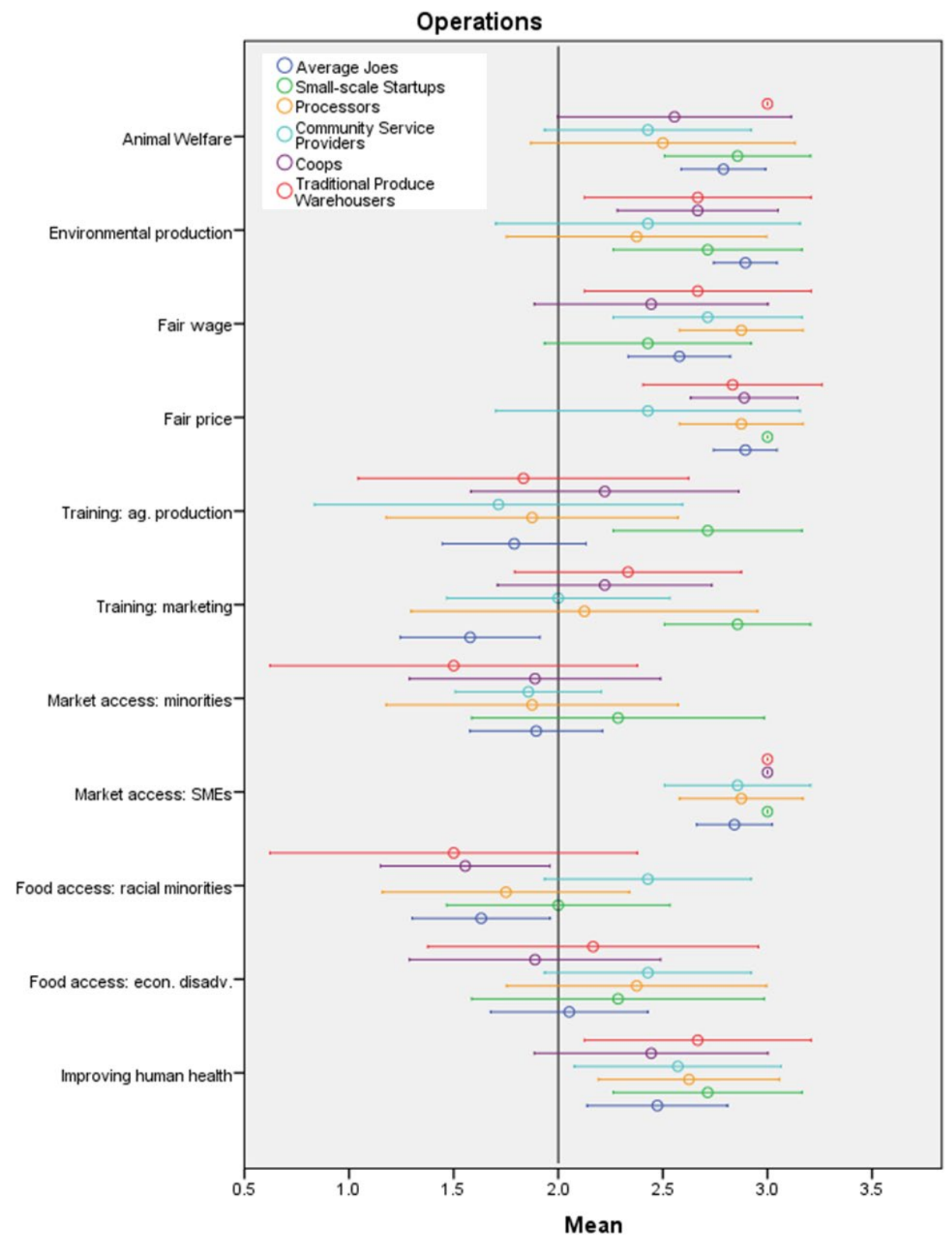

Error Bars: $95 \% \mathrm{Cl}$

Source: Author.

Note: Ag. = agricultural; Econ. disadv. = economically disadvantaged populations; FHO = food hub organization; SME $=$ small- and medium enterprise. 
Table 5.3 Mean Differences in Relevance of Social Values to Mission and Day-to-Day Operations Variables, With Each Species Compared With All Other Observations

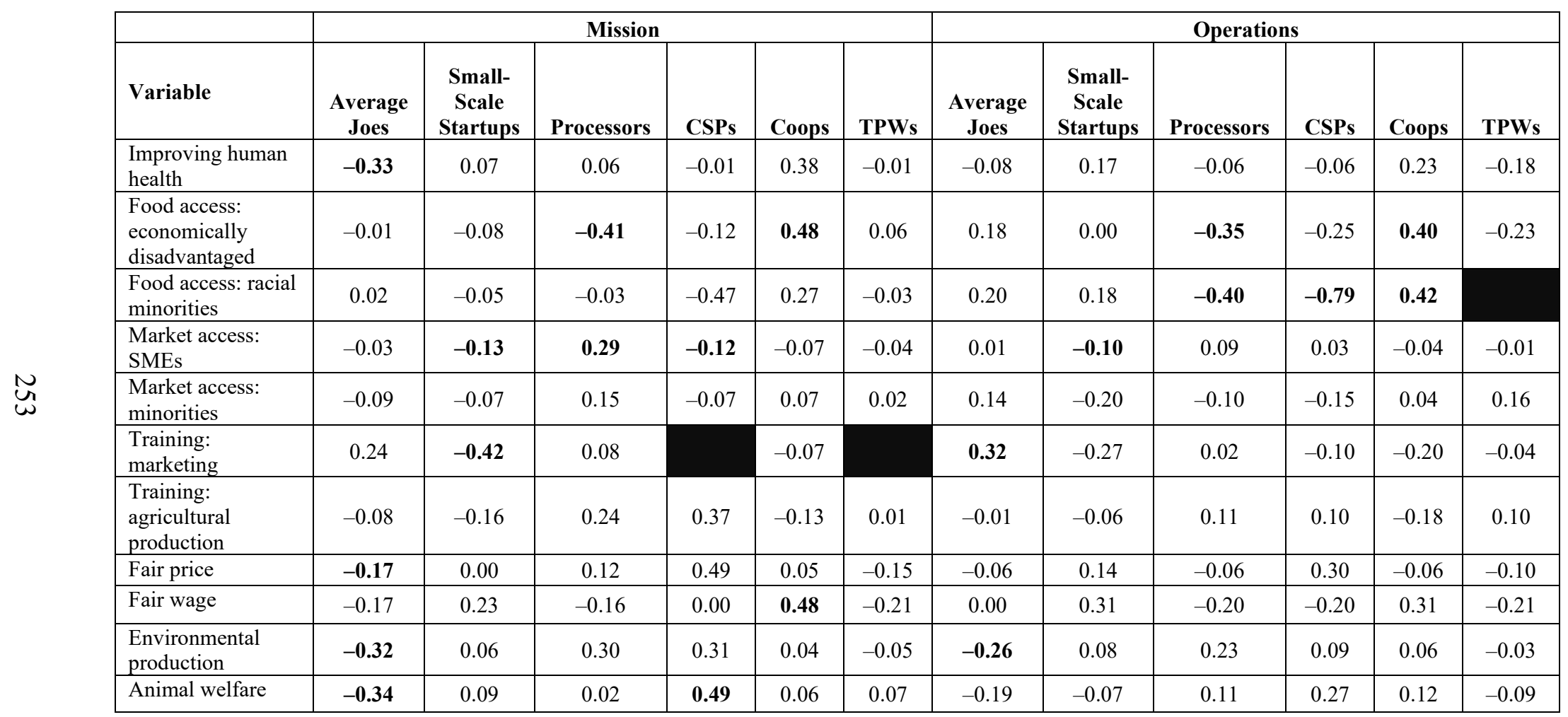

Source: Author's calculations.

Note: Value in bold indicates statistical significance at or beyond the 10 percent level. CSP $=$ Community Service Providers; TPW $=$ Traditional Produce Warehousers. 
Table 5.4 Regression Analysis Results for FHO Performance Variables

\begin{tabular}{|c|c|c|c|c|c|c|c|c|c|c|c|c|c|c|c|c|c|}
\hline & \multicolumn{8}{|c|}{$\begin{array}{l}\text { Total Value of Product Moved } \\
\text { (in hundrend thousands) }\end{array}$} & \multicolumn{8}{|c|}{$\begin{array}{c}\text { Profit } \\
\text { (in hundrend thousands) }\end{array}$} \\
\hline & & B & $p$ & B & $p$ & B & $p$ & $\mathbf{B}$ & $p$ & $\mathbf{B}$ & $p$ & B & $p$ & B & $p$ & $\mathbf{B}$ & $p$ \\
\hline \multirow{6}{*}{$\begin{array}{l}\frac{\mathscr{U}}{0} \\
\frac{\mathscr{L}}{2}\end{array}$} & Intercept & 1.07 & 0.70 & 2.52 & 0.50 & -2.64 & 0.40 & -13.24 & 0.12 & 0.23 & 0.91 & 0.03 & 0.99 & -0.68 & 0.82 & -12.19 & 0.12 \\
\hline & Small-scale startups & -0.91 & 0.86 & -5.10 & 0.37 & -4.78 & 0.33 & -3.06 & 0.64 & -0.21 & 0.96 & -5.03 & 0.23 & -5.69 & 0.23 & -4.37 & 0.48 \\
\hline & Processors & 2.72 & 0.56 & 1.34 & 0.79 & 3.37 & 0.40 & 0.93 & 0.85 & 0.38 & 0.92 & -0.85 & 0.81 & -0.63 & 0.87 & -5.10 & 0.27 \\
\hline & Community service providers & -0.52 & 0.93 & -2.17 & 0.74 & -0.47 & 0.93 & -1.96 & 0.78 & -0.14 & 0.98 & -2.23 & 0.64 & -1.87 & 0.71 & -3.85 & 0.56 \\
\hline & Coops & 7.44 & 0.10 & 6.81 & 0.14 & 7.60 & 0.06 & 9.27 & 0.09 & 7.15 & 0.04 & 6.44 & 0.06 & 6.64 & 0.08 & 8.72 & 0.10 \\
\hline & Traditional produce warehousers & 6.35 & 0.21 & 4.19 & 0.42 & -3.92 & 0.36 & -3.02 & 0.51 & -0.26 & 0.95 & -2.61 & 0.49 & -3.72 & 0.37 & -3.02 & 0.49 \\
\hline \multirow{6}{*}{$\begin{array}{l}\text {.0 } \\
\stackrel{0}{00} \\
\stackrel{0}{\simeq}\end{array}$} & Southwest & & & -1.04 & 0.84 & 0.98 & 0.83 & 2.82 & 0.61 & & & 2.09 & 0.57 & 2.41 & 0.58 & 4.20 & 0.42 \\
\hline & Northwest & & & 10.36 & 0.08 & 14.46 & 0.00 & 18.82 & 0.00 & & & 13.68 & 0.00 & 14.50 & 0.00 & 17.73 & 0.00 \\
\hline & Midwest & & & -3.96 & 0.47 & -0.31 & 0.94 & 3.65 & 0.47 & & & -0.90 & 0.82 & -0.43 & 0.92 & 2.98 & 0.53 \\
\hline & Gulf states & & & -0.89 & 0.91 & 0.90 & 0.89 & 10.09 & 0.36 & & & 2.05 & 0.71 & 1.46 & 0.82 & 7.93 & 0.45 \\
\hline & Southeast & & & 0.40 & 0.94 & 4.56 & 0.28 & 5.93 & 0.21 & & & 0.85 & 0.82 & 1.49 & 0.71 & 3.35 & 0.45 \\
\hline & Great Lakes states & & & -4.32 & 0.39 & -1.79 & 0.66 & -5.13 & 0.26 & & & -1.08 & 0.77 & -0.84 & 0.83 & -4.49 & 0.29 \\
\hline \multicolumn{2}{|r|}{ Total number of suppliers (est. max.) } & & & & & 0.05 & 0.00 & 0.07 & 0.01 & & & & & 0.01 & 0.35 & 0.03 & 0.19 \\
\hline \multirow{15}{*}{ 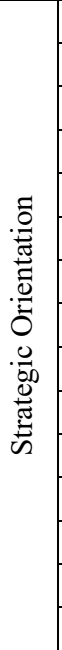 } & Total community service types & & & & & & & 1.19 & 0.15 & & & & & & & 1.43 & 0.07 \\
\hline & Retail channels \% sales & & & & & & & 0.06 & 0.27 & & & & & & & 0.04 & 0.41 \\
\hline & Intermediary channels $\%$ sales & & & & & & & 0.11 & 0.31 & & & & & & & 0.06 & 0.53 \\
\hline & Institution channels \% sales & & & & & & & -0.08 & 0.49 & & & & & & & -0.08 & 0.47 \\
\hline & Processed Produce & & & & & & & -0.02 & 0.88 & & & & & & & 0.07 & 0.51 \\
\hline & Meat & & & & & & & 0.11 & 0.08 & & & & & & & 0.14 & 0.02 \\
\hline & Fish & & & & & & & -1.69 & 0.44 & & & & & & & -2.33 & 0.26 \\
\hline & Milk and dairy & & & & & & & 0.01 & 0.94 & & & & & & & 0.03 & 0.85 \\
\hline & Eggs & & & & & & & -0.58 & 0.07 & & & & & & & -0.54 & 0.08 \\
\hline & Dry goods & & & & & & & 0.06 & 0.63 & & & & & & & 0.06 & 0.61 \\
\hline & Baked goods & & & & & & & 0.97 & 0.26 & & & & & & & 0.87 & 0.28 \\
\hline & Coffee and tea & & & & & & & -0.78 & 0.73 & & & & & & & -1.11 & 0.60 \\
\hline & Other value added & & & & & & & 0.12 & 0.33 & & & & & & & 0.14 & 0.22 \\
\hline & Nonfood & & & & & & & -3.24 & 0.11 & & & & & & & -2.90 & 0.13 \\
\hline & Unclassified products & & & & & & & 0.24 & 0.48 & & & & & & & 0.20 & 0.54 \\
\hline \multicolumn{2}{|c|}{ Adjusted $R^{2}$} & \multicolumn{2}{|c|}{-0.01} & \multicolumn{2}{|c|}{-0.01} & \multicolumn{2}{|c|}{0.41} & \multicolumn{2}{|c|}{0.43} & \multicolumn{2}{|c|}{0.01} & \multicolumn{2}{|c|}{0.08} & \multicolumn{2}{|c|}{0.07} & \multicolumn{2}{|c|}{0.14} \\
\hline
\end{tabular}

Source: Author's calculations

Note: Reference group for species is Average Joes; the reference group for region is Northeast. FHO = food hub organization. 


\section{CHAPTER 6 \\ CONCLUDING REMARKS}

I intentionally limited the scope of this dissertation to a single node in the network of economic agents that comprise short food supply chains (SFSCs) in the United States. These SFSC networks form local and regional food systems (LRFSs) and the node I study in this work is the set of coordinating intermediaries know as "food hubs." This set of organizations are often identified as being highly heterogeneous in their structures and strategic behaviors; as one trope in the food hubs policy space contends, "if you've seen one food hub, you've seen one food hub." In this work, I investigate whether these emergent organizations are best characterized as a single population with one nascent organizational form or whether there are several populations of distinct natural kinds. Investigating this question is important not only for foundational scientific inquiry on the evolution of organizational forms but also for applied policy—these food hub organizations (FHOs) fill the opportunity space created by the local and regional foods movement (LRFM) with the promise of building capacity to increase LRFS scale.

In this chapter, I summarize and describe the findings and limitations of my empirical work. I also present a number of avenues to extend the work of the preceding chapters- either from a resolution of one or more of the limitations I encountered or from de novo inquiry. Those extensions lay out a multifaceted possible research stream that promotes knowledge at the applied and foundational science levels. I close with some brief remarks on this dissertation's overall contributions.

\subsection{Findings}

The results I presented in Chapters 4 and 5 provide a proof of concept for the organizational species concept (OSC) to classification. These results also highlight how 
applying the OSC can construct an ecological approach to understanding economic systems. Thus, the empirical findings of this dissertation provide not only an example of classification but also represent a preliminary investigation into the ecology of LRFSs. Such an ecological approach can inform policy decisions about the management of foodsheds to achieve goals such as scaling up. It helps us understand, for example, what is being scaled up - the individual agents, the system itself (that is, sets of transactional arrangements that produce, transform, and distribute food products with the given credence attributes), or both. My findings also shed light on concerns voiced in the literature on local foods about trade-offs between scalability and the tenor and magnitude of socially embedded transactional environments, agential behaviors, and activities that build the social fabric sought by the local foods movement. Overall, the findings I present in Chapters 4 and 5 indicate that taking an ecological approach to managing economic systems — one that incorporates interventions that build biodiversity in the environmentis likely the best means of alleviating this tension and achieving the seemingly juxtaposing goals of social investment and scalability.

\subsubsection{Outcomes of Classification}

By applying the OSC to data on FHOs from the 2015 National Food Hub Survey, I find that distinct patterns of morphological attributes exist within and among the observed organizations and that these patterns are associated with statistically significant differences in strategic orientations. The finding of distinct patterns indicates that there are natural kinds of FHOs (in terms of morphological structures) while those indicating strategic differences validate these kinds as meaningful and thus allow us to accept them 
as species—as distinct organizational forms. ${ }^{80}$ Under hierarchical cluster analysis procedures using Ward's method of linking, my classification work identifies six such species among FHOs. In identifying these organizational forms, this work allows me to characterize each species' population in terms of relative size, the morphological difference makers, which delineate them from others, the variance within these morphological characteristics, and the strategic niche and functional roles occupied within the local foods opportunity space each holds.

My results indicate a predominant or common species - that which is most widespread and with the largest population, which I term Average Joes. Organizations of this form hold what can be considered as a baseline of FHO characteristics. They tend to be for-profit firms whose revenue predominantly comes from the sale of agrofood products for which they take ownership (that is, they do not act as brokers). They offer services to suppliers in terms of additional packaging beyond the farm gate and transportation of product to the hub. Some hold retail space to sell their products, and about half have an online ordering system—otherwise, sales are completed largely through personal relationships with buyers, sold out of very small warehouses and delivered with a small fleet of hub-owned vehicles. They rely highly on sales of fresh produce and sell primarily to buyers in the retail and restaurant sector, thus fulfilling a role as intermediaries between farmers and high-value buyers who require coordination. About half of their suppliers have been in operation for fewer than 10 years, and less than

\footnotetext{
${ }^{80}$ In other words, despite relative heterogeneity among food hubs, characteristics are not likely to be distributed in a free-for-all, but rather occur in relative coincidence with reason.
} 
30 percent are owned by women or people of color (that is, those who are considered socially disadvantaged).

Similar to Average Joes are TPWs, who similarly rely on product and channel strategies and thus fulfill a similar functional role. These TPWs, however, operate at a much larger scale and tend to provide rental space in their facilities to other food businesses. Managers of TPW FHOs have the lowest average rating for experience in or knowledge of agricultural production. Evidence from qualitative investigation indicate that these TPWs may have a different evolutionary path than do Average Joes and may be engaged in a form of mimicry, moving from conventional agrofood markets into the opportunity space created by the LRFM and its imaginary. TPWs are a much smaller population of the sample. The other four species focus primarily on sales directly to consumers, but each still fills a specific niche in the local foods landscape. They are differentiated morphologically along various lines, which affect the niche they inhabit.

Small-scale Startups are younger organizations and more likely to be established as nonprofits. They have low levels of capitalization with very little or no warehouse space or vehicles on hand, are not likely to have a storefront or processing facility, and do not offer transportation services to suppliers. Their managers have the lowest average ratings for knowledge and experience levels in management and warehousing operations. They offer few community services and — similar to most food hubs—-focus product offerings on fresh produce with some meat and poultry. These are very similar to community-supported agriculture schemes. In contrast, there are processors and Community Service Providers. Processors are the most likely to hold processing facilities and are engaged in more packaging on-site. It is fitting that they rely the most on 
processed produce and value-added categories, although these are still marginal compared with fresh produce. CSPs gear heavily toward engagement with consumers, especially in educational programs and other activities that stimulate latent demand for local foods. These organizations are the smallest population among the species of FHOs. They are most likely to offer products under a brokerage role and to have a kitchen facility that is licensed and available for shared use, to offer rental space to others, and to have a retail space of their own. Both species have a higher degree of reliance on nonsales revenue and higher proportions of suppliers who are socially disadvantaged; also, their managers have the highest average ratings for experience in or knowledge of agricultural production.

Meanwhile, the final species—Coops—does almost no community services geared toward the consumer end of the supply chain and likely fulfills a role as a service provider to supplier-members. However, these supplier-members are also predominantly White and likely male, with Coops having the lowest average percentage of suppliers from socially disadvantaged backgrounds. Their managers are inexperienced, relative to managers of other FHO species; Coops have the lowest average rating for knowledge and experience in the key function categories of processing, strategy, and other and are in the bottom two across other functional areas. These organizations have the highest reliance on meat and poultry products (although it is still a minority to fresh produce). They are least likely to engage in additional packaging services for suppliers or to have a processing facility but, I infer, are likely fulfilling some role in accessing small-scale abattoir services for their members given their reliance on meat and poultry. 
I draw these characterizations using mean profile values and the magnitude of mean differences (as well as whether these differences are statistically significant); they represent the average or typical proto-member of a species - a representative abstraction of the species' population. Empirical results under the approach I develop to operationalize the OSC also allow us to understand the variation within and between species. This information comes from an analysis of variance and independent-samples $t$ test analysis and is available for strategic, performance, and morphological data points. Some of this information as error-bars graphs in the figures and as quantitative measures in the tables in Chapters 4 and 5. More detailed quantitative data are available but not provided directly in this dissertation. My results broadly show that variation among and within any given FHO species is high. This is especially so for certain attributes, often those common to the food hub genus (such as reliance on fresh produce and sales to direct-to-consumer and direct-to-retailer-and-restaurant channels or the presence of an online ordering system as part of the firm's structure). This general condition of high variation is exemplified, for example, in the 95 percent confidence intervals of the errorbar charts.

However, this variation can be idiosyncratic. Species with small relative population sizes exhibit larger variation because of small sample sizes (that is why data for CSPs is often observed to have broad confidence intervals). Other times, we must consider that this variation may be important in relative terms; what does it mean, for example, if most species have very little variation for some given variable, whereas among one single species' population there is a high degree of variation in that element? This itself may be a meaningful difference maker because it indicates adaptability of the 
species' morphology to environmental conditions or that said attribute is not a difference maker for that species, whereas others are. Because of this, I emphasize characterizing species using mean profiles for this work. However, it is an important outcome to note for its value to future applications of the OSC.

This leads to another important outcome of this work: difference makers- that is, attributes that drive the boundaries between species - are idiosyncratic not only to the species but to the comparison. Under the polythetic approach inherent in the OSC and its method of operationalization, the difference makers for any given species may not be universal across all species of a "genus" or "family." In other words, the divergence in attributes between any two species may not be the same as those that define the boundaries between any other two species. This polytheticism likely explains the dispersion we observe in the reduced dimensionality of the multidimensional scaling coordinate space for FHO species (see Figures 4.5 through 4.8 and 4.11). Recall that these graphics represent approximations of how the cases - here, individual organizations - relate to one another in multivariate space, but with certain variables concatenated together to visualize this in a way that humans can easily perceive; if several attributes act as difference makers across species or if difference-making patterns are relative to populations under comparison, we are bound to observe overlaps or noise as we squeeze these dimensions together into two- or three-dimensional space.

This dispersion may also be because a given group is characterized by relative heterogeneity (that is, high variance) in many morphological attributes but consistency in others, especially those that are rare among other populations. This is only one expression of "relative coincidence." Furthermore, reduction of the multiple dimensions contained 
within the set of morphological characteristics down to 3 or 2 dimensions of visualizable Euclidean space means that idiosyncratic difference makers- those attributes that diverge between, for example, only two species - may be lost in the visualization but still present in the underlying data. In the validation stage, we examine the effect of these small differences through the effects on outcomes, such as strategic niche. This kind of fineline alteration is seen in biology; in the classical case of Darwin's Galapagos finches, species are notably delineated on their beak shape and size, but for which there are also less noticeable differences in morphology. The beak, especially, affects their functional role in the ecosystem and the relevant resources they access. Several of these species may overlap in the same ecosystem, despite all being finches that are otherwise very similar except for the difference makers. My application of a species concept to food hubs - and the attendant empirical results — provides clear evidence of similar phenomena among organizations; differences in the relative coincidence of morphological attributes-even small ones - are associated with changes in behavior, outcomes, and functional role within the marketplace.

When we find evidence that the relative coincidence of morphological characteristics within a population of firms is associated with that population having distinct behaviors, accessing specific resources, or fulfilling differentiated functional roles, that population should be considered a distinct organizational form. As economists, we seek to understand complex econosystems to predict how changes in the market environment may affect societal goals. This is the purpose of proper organizational classification—cogently and robustly identifying organizational forms in order to investigate their role in markets. In Chapter 5, I perform such an analysis, and the 
understanding we gain about the functional roles of FHO species is another outcome from classificatory work. As an astute reader may surmise, these functional roles extend beyond the strategic orientations used to proxy the organizational niche deployed in Chapter 4 to validate the natural kinds of the final postulated cluster solution as species. Knowledge of the functional roles played and connections formed by given organizational forms, especially when combined with knowledge about population sizes and geographies, is what makes the classification meaningful to economic science.

\subsubsection{Policy Implications}

Our understanding of the functional roles that empirical results in this work show different organizational forms play within LRFSs has implications for policy decisions that seek to manage these systems and the organizations within them. Findings from Chapter 4 indicate that FHO species inhabit different market niches through distinct strategic orientations in terms of the downstream connections they make which form short food supply chain networks, the products they sell through these networks, and their effect on communities through social engagement. Meanwhile, investigation in Chapter 5 provides evidence that the functional roles of the different species extend beyond the strategic orientations to include the number and type of primary producers accessing local foods opportunity space through FHOs. Different species also face different managerial and financial landscapes. These differences are related to population boundaries drawn between the natural kinds of FHOs on the basis of polymorphic attribute patterns - not every morphological trait must be present to the same degree in any individual organization. In other words, we can allow for fuzzy boundaries when considering any given FHO, but know that certain relative structures are likely to affect the functional role of the firm within a given market space. 
It is crucial that these different functional roles address the scaling-up challenge from distinct vantage points. Numerous examples abound. Processors make capital investments that create value-addition opportunities for their suppliers, thus scaling-up through value creation and, it is assumed, passing this value on to suppliers. Coops appear to address failures in the market for abattoir services - especially for small-scale livestock producers and for the preservation of the local foods movement's imaginary and associated economic value - while increasing margins and the return of captured value to member-owners (which are often farms and ranches, but may also be consumers or other downstream agents). TPWs operate on large scales, offer rental space in a collective setting for other agrofood firms, and address overall system scale (likely by providing a market for either a larger number of primary producers and/or for those of larger scale). CSPs focus on issues of equity and justice. They engage with final consumers, likely entering food deserts and addressing the needs of at-risk populations to expand access and capacity to consume more nutritious foods and address other livelihood issues. They also purchase at much higher rates from socially disadvantaged and new and beginning suppliers. This means that not every individual FHO should be expected to address every desired outcome or fill every possible role within its market space. One unpublished case study conducted on background from this dissertation is a good example of how an FHO may have a "crisis of identity," overextend itself and thus fail to thrive.

This finding about functional roles being tied to the relative coincidence of morphological characteristics of FHOs is important for a number of key stakeholder groups; it can guide foodshed managers and practicing agents, those leading the local and regional foods movement and crafting its imaginary, those making agricultural and food 
policy, and researchers and extension agents investigating food systems and providing knowledge resources and crafting interventions. The level at which one of these stakeholders is operating becomes important to a certain extent, but the general knowledge of FHO species has broad appeal across levels. Particularized assessment of the space in which a stakeholder is operating is key to how the OSC's application to food hubs can assist them in decision making.

For example, the manager of a given foodshed — such as a local community's food policy council—should perform a detailed assessment of their particular community to identify strengths and weaknesses, opportunities and challenges, within their foodshed. With this knowledge, they can identify community priorities and deploy the findings from this work to stimulate the creation FHOs within their foodshed with organizational forms more likely to address those needs. Sharing this knowledge with food system practitioners - including FHO managers, primary producers, and community membersmay empower them to shape the organizations they establish or with which they engage. Investments can be targeted toward capital assets, programs, labor resources, and other structural elements within existing or newly established FHOs. These decision makers know that such investments cannot be in a single characteristic but likely need be in key complementary facets of these organizations. Moreover, organizational adherents have benchmarks by which to judge the performance and identity of the FHOs with which they interact.

These findings inform local foods movement activists and leaders on the way different organizational forms may enact the imaginary of the social movement and their contribution to enacting movement principles in the opportunity space it creates. 
Concerns frequently raised in the literature on local foods about trade-offs between increasing scale and social action are partially supported by my findings; certain FHO species with improved economic performance and larger total volumes provide, on average, fewer services to the community. At least, that is, to the consumer-segment of the community. However, that this concern is supported does not mean that scaling-up LRFSs cannot or should not happen; as my results also show, different species fulfilling different roles enact a "commitment to place" in different ways. Coops and TPWs, for example, likely enact this commitment through the promotion of specific supplier segments. If racial and gender justice are of concern to local movement leaders, building the capacity of organizations to have more inclusive supplier networks can improve the equity and justice of these marketing channels. These findings empower movement organizers to have discussions about what the imaginary of local foods is for their place, and to militate for organizational forms — and thereby open opportunity space - that suffice these desires. Tensions between the economics of scale provision and social responsibility can be addressed through managing food systems as ecologies, building market infrastructure (that is, organizations) that are more likely to fill the desired niches. So too can the movement place pressure on community leaders and elected officials to create policies that promote these goals.

In turn, these policymakers can use these findings to assess the wider environment in which one or more foodsheds under their jurisdiction exist. Those seeking, by example, to build regional food systems or to pursue an Agriculture of the Middle paradigm (that is, create opportunities for economic viability of mid-sized family farms) may seek policies that incentivize existing distributors to move into source-identified 
markets. Others wishing to stimulate startup activity and new business formation broadly may direct resources to programs that support the establishment of small-scale FHOs that can grow naturally and scale up food systems by creating more stable distributional channels for new and beginning and small farmers. The results I present here on the different species can inform policy realms such as taxation, food safety regulations, cooperative organizational law, investments in extension and business incubation programming, and others.

My findings inform those of us working in research on organizational dynamics within food systems and in the translation of that research through cooperative extension outputs. It tells us that we cannot treat all organizational populations as homogenous (even if the assumption is of "homogenous heterogeneity." We must perform applied research that accounts for organizational forms and distinct organizational populations. I discuss in a subsequent section some avenues of research where my work here opens the door on this front. When crafting extension materials and offering support to industry and community leaders through consultative functions, extension agents and specialists must help clients assess their environment and the functional roles they need filled, and then identity the set of complementary organizational features most likely to succeed.

From the preceding paragraphs, however, another policy implication of my work here arises: how we define and parameterize scaling-up matters. Is scaling-up about stimulating a larger number of supplier firms? Is it about bringing more suppliers into local foods space? Is it about increasing the average size of supplier firms within local and regional marketing channels? Is it about building inclusive opportunity for minority, marginalized, and socially disadvantaged firms? Is it about selling to a greater number of 
customers? Or including more kinds of customers in the local foods market? Is it about product volume? Or total value? As it turns out, the classification work I conduct here matters for the kind of scaling-up targeted by policy makers and stakeholders in a foodshed. As my results show, a given species of FHO is likely to achieve different kinds of scaling up. Coops expand products and capture more value for supplier-members, but appear to lack the kind of inclusive growth many urban food system planners desire. CSPs and Processors do better at inclusion but struggle financially and may not create large amounts of value for suppliers. TPWs appear to move large volumes and likely serve buyer markets that would otherwise struggle to purchase local foods but may sacrifice core elements of the imaginary created by the LRFM to do so. Approaching the scaling-up demand from a wider lens, findings from this work and qualitative research done in support of it indicate that FHOs are—writ-large—-generally about solving problems in thin markets and "letting small-scale work" through coordination and intermediation.

\subsubsection{Methodological Import}

The methodological approach I develop to operationalize the OSC has as a general form of (1) search for patterns within attribute data of cases, (2) place cases into groupings based on the underlying patterns discovered among the attributes, and then (3) determine whether those groupings are associated with meaningful differences in outcomes and thus whether the patterns — which the groupings represent—should be accepted or rejected as organizational forms and the set of cases within these groupings considered as distinct populations. In the empirical work of this dissertation, I deploy HCA as the tool for searching the data to find patterns. HCA reduces these patterns to Euclidean distances, placing cases in relation to one another and then linking them—or 
the groupings to which they have been assigned—in accordance with some rule (algorithm). Here, the rule applied that yields the six species of FHOs is Ward's method, which prioritizes linking so as to ensure that grouping causes the minimum possible increase in total variance within groups. HCA is the established and accepted starting point for exploratory classification. It is preferred when there are no theorized groupings a priori, or when such a priori categorization is not philosophically desired.

In the context of classifying FHOs, both elements are at play. Early work in organizational systematics, highlighted in Chapter 3 , indicates that to appropriately engage in numerical phenetics and create organizational classification schemes, we should adopt an emergent approach to category formation. We should avoid holding a theory of a priori groupings and instead allow categories to arise from patterns we observe within the data. Furthermore, there is effectively no extant categorization of food hubs, especially not one which is based in quantitative empirics. It is noteworthy that HCA is not the only methodological tool to use to search for patterns among data on cases. In subsequent sections of this chapter, I identify additional empirical tools that can be deployed in the search for these patterns and formation of categories. This may commence only now that my work has provided a preliminary set of species, developed under an emergent and empirical process using HCA. These additional tools hold promise to refine the categorization of cases into organizational forms and to more clearly define the boundaries of the organizational populations. Their application will also support or refute my findings, thus continuing the scientific process of category formation and testing. 
And this is the benefit of the methodological approach I provide here: because it includes a validation stage on ex post variables, one implication of my methodological approach is that we can quantify and empirically test the effect of refined search parameters and alternative categorization of case firms on outcome variables. As just one example, in my work I use Ward's method under hierarchical linking, which is mathematically related to nonhierarchical kMeans procedures. Using outcomes from this dissertation, I will be able to apply seeded kMeans, using the mean profiles from this analysis as the seeding kernels, to refine the six-species solution. I can then measure the difference in the sum of squared errors between categorization under Ward's HCA and kMeans procedures for the set of both morphological and strategic variables. This can provide insight about how additional categorization methods "tighten" the variance within groupings.

On a final note, the methodology I adopt here cannot overcome a number of limitations, especially those that arise from data completeness and quality. I discuss a variety of limiting factors in the subsequent section in greater detail. Of import here is that the inability to overcome these through methodological means-including improved data collection, imputation, modeling techniques - implies that there are simply some things we do not know under the current study. For example, we do not know the degree to which the assignment of cases to groupings - and thus the resulting species boundaries - might change where more information available for inclusion in cluster analysis stages. This could be in the form of additional variables of morphological characteristics, the inclusion of additional cases, or simply greater richness in the measures used. Relatedly, we also do not know how accounting for additional exogenous 
variables that measure environmental factors may affect results from the validation stage's regression analysis. Furthermore, we do not know for certain if the firm properties I include as morphological variables are relatively homeostatic. For this analysis homeostasis is an assumption-one well-grounded in the economic theory of those properties, but an assumption nonetheless. Our estimates of population sizes are approximations based on relative incidence among the sample; we do not know if there are response biases that cause certain kinds of FHOs to respond to the survey instrument at rates lower than their actual proportion in the population or if there are species of organizations that act as FHOs but do not outwardly identify themselves as such. Last, we know very little about the supplier firms and thus lack knowledge as to whether certain FHO species are associated with specific supplier types.

Overcoming these known unknowns would allow us to have a truly McKelveyian approach to phenetics - one in which a deep, deep set of organizational attributes across a large sample of the population is considered and sorted into natural kinds. This kind of phenetics may simply not be plausible with organizational populations, as the methodological requirements in terms of data collection are too onerous for the organizational subjects we study. Unlike our colleagues in biological sciences, those of us in economics and organizational science cannot dissect in fine detail large numbers of specimens. Thus, my work here provides another methodological implication: as social scientists our classificatory work may never be as detailed and precise as that of the biologist, chemist, or physicist. We must adopt methodologies that allow us to approximate as best we can but to be more accepting of category switching and updating in our classificatory scheme. 


\subsection{Limitations Encountered in this Study}

The literature on organizational ecology and organizational systematics has consistently identified data requirements as the primary limitation to empirical work on organizational classification; McKelvey, for example, identified this early on, and concerns have been pernicious throughout work across the pertinent fields. The intensity of data needed to appropriately classify populations of organizations poses logistical problems in the collection of information about firms in any single survey, let alone the creation of a proper linked panel data set that spans long temporal periods. Even if a perfect data set were available, estimation methods also limit the progress of empirical analysis. Although substantial improvements have been made in the ability of statistical algorithms and computing systems to handle such large and complex data, there are still no universal subroutines that allow rapid, automated investigation of multiple clustering approaches, nor statistical artifacts that enable their comparison. This study is no different than others in the field: I encountered a number of limitations related to data and empirical methods in the work that I complete here to apply my organizational species concept.

\subsubsection{Data Quality and Completeness}

Primary among these limitations are issues with the completeness and quality of data available for food hub organizations. Despite the 2015 National Food Hub Survey being a novel dataset of national scope capturing a large proportion of the expected population, its design and collection led to a number of limitations. The survey instrument was exceptionally long and was not enumerated in the field by professional researchers; instead, food hub manager respondents had to complete it on their owneither digitally or on paper. The design of certain questions and data entry fields is 
problematic in portions of the survey, and it does not appear simple logical enforcement tools were used to validate respondent entries in some of these complex areas. As an example, one section asks about labor resources within the firm. Respondents are solicited for information on the total numbers of staff across various categories of employment (for example, managers, full-time staff, part-time, volunteers). This solicitation is presented in the form of a table with two additional columns - one to provide the number of staff in that category who identify as women and one for people of color. For a large number of observations, this information is problematic, with values for the two demographics questions summing to more than the total, values entered for demographics columns but not the total column, and so forth. Similar problems with data quality were observed in other elements of the survey. These issues, stemming from poor survey design, meant that entire information sets—realms I believe would be important "difference makers" in classification—had to be omitted.

Moreover, the lack of enumeration—on a survey that had a manageably small number from the start—meant that respondents skipped entire sections of the survey. There is anecdotal evidence of respondent fatigue, with item response rates dropping the deeper into the questionnaire one goes. These completeness ${ }^{81}$ issues also mean the omission of data elements as well as the need to drop cases, thus reducing the total sample size and effective response rate for my work here. The limitation on effective sample size is likely a driver of the large confidence intervals observed in comparisons of species in terms of morphological and strategic variables. It reduces the statistical power

\footnotetext{
${ }^{81}$ The current state of the art in clustering methodologies does not allow for imputation of missing data. However, researchers in statistical methodologies are actively working on improvements to clustering algorithms that would allow for advanced methods such as imputation and sample weighting.
} 
of the observations I make. Furthermore, because the 2015 National Food Hub Survey was not designed for this specific use, some data elements were not collected in sufficient detail. This affects cluster analysis methodologies and their outcomes (for example, the need to use dichotomous or categorical variables alongside those on continuous scales). This is an unfortunate reality for much of the work in organizational classification where the kind of data intensity needed for phenetic (that is, morphological attribute-based) approaches to HPCs means we must often rely on secondary data sets as I do here.

\subsubsection{Methods and Stage of Research}

Additional limitations arise simply from the stage of the research on the OSC; here I provide a proof of concept and in future more refined and specific methodologies may be adopted that improve upon and elucidate the power of my conceptual contribution and initial empirical work. Improvements in data availability offer one avenue to reach beyond a methodological limitation encountered here: I had to account for homeostasis of the property clusters (that is, firm attributes) implicitly. This is because I lacked access to a panel data set that collects information on the morphology of FHOs over time. This was an allowable expense given that the applied science needs to understand how FHO species may play a role in scaling up LRFSs and given the newness of the organizational form. Data for versions of the National Food Hub Survey conducted before and after the 2015 version used here were not made available. There is an open question as to the cross-iteration responses rates, with an expectation of low response rates from the same organizations over time. Moreover, primary data collectors were unable or unwilling to provide geographic indicator information — such as zip codeswithin the set provided to me. This also provided methodological and modeling limitations as environmental data of any value could not be incorporated into the 
analyses. This would have been especially important for regression analyses in Stage 3 of the method I develop for operationalizing the OSC. Its inclusion would provide more robust analysis in future studies to validate effects on strategic orientations of firm classifications and thus acceptance as species.

In developing that operationalization method, I also note that HCA is the necessary (and proscribed) first step in initializing clustering approaches to understand patterns within data. Other algorithms need to be incorporated as this body of work continues, once HCA and other initial data investigation steps indicate the likely presence of distinct natural kinds within a given population. This will especially include nonhierarchical means of cluster formation such as kMeans, kMedioids, density-based models (such as DBSCAN), and (Gaussian) mixture models (which are statistically based means of forming cluster assignments). However, this is the benefit of the OSC and operationalization method I develop in this dissertation: the conceptual framework is flexible to alternative empirical specifications that can create more refined understandings of the underlying structure of a population's attributes. Applying these additional methods to data on FHOs, for example, is likely to refine the cluster assignments and provide clearer boundaries between species. This refinement may reduce (or increase) the number of species from the groups I accept as such in this work, which is the natural and good course of scientific investigation into systematics. One need only review the history (and heated debate) in biological systematics — or even planetary systematics (think: is Pluto a planet?) — to know that this is the expected progression of improvements to classificatory work. Classification schemes are malleable to an extent, 
and overcoming modeling limitations I encounter in this proof of concept will lead to better assurance about distinct populations of FHOs within LRFSs.

\subsection{Extensions and Future Research}

It is important to identify where the developments I make in this dissertation can lead us. In the course of my work on developing the OSC in the context of FHOs, I have identified a number of extensions to be made with that organizational population itself. I also identify and have begun the work of extending the OSC's application to other populations of organizations with different functional roles within LRFSs. These extensions provide room for new developments under applied science with policy relevance, as well as for foundational science in terms of methodologies and theoretics.

\subsubsection{Extensions With FHOs and Other Organizational Populations in LRFSs}

A number of extensions with FHO populations are immediately apparent from the discussion in section 6.2.1 and 6.2.2. Overcoming data limitations would provide for more robust clustering and validation models. Accessing panel data-even with some effective sample size issues - would allow the work I complete here to extend beyond an implicit assumption of homeostasis in the properties and account for it explicitly. The National Food Hub Survey collection has been done every other year since 2013, which

provides — theoretically — a four-set panel covering the past six years of population life. Having this panel data set would also allow for investigation of population dynamics at a detailed level and may enable extensions into hazard analysis and firm failures. Do certain species fail to thrive? Do shifts in habitats cause adaptation over time? The questions abound and a rich research stream emerges.

Application of the OSC to other populations to continually prove the usefulness of the concept is also a key extension of the work I initiate in this dissertation. Building the 
research stream on organizational classification in this regard has already begun. In a forthcoming working paper, I present results of applying the OSC to data on primary agricultural producers - that is, farms and ranches - in LRFSs. That forthcoming work adopts the same methodology I do here to analyze the 2015 Local Foods Marketing Practices Survey, a census-level data product from the USDA's National Agricultural Statistics Service. In that work, I promote an eight-cluster solution as a species set, and show that certain morphologically determined species select into strategic mixes that prioritize the use of intermediated marketing channels (that is, FHOs) and that those species are overall larger in terms of farm size and gross sales. I interpret this as evidence supporting conclusions that FHOs support scaling up. It is important to note that that work led to an additional extension investigating the equitability of local marketing channels for socially disadvantaged primary agricultural producers - those firms whose primary operators are women or people of color. Results there indicate that socially disadvantaged primary producers do not access FHO channels, which corroborates observations in this dissertation that only a select population of FHOs - the smallest total population among the species set—have notable rates of socially disadvantaged suppliers. This highlights how extending the OSC to other elements of LRFSs provides a clearer picture of how these econosystems function and provides critical knowledge that can be used by policymakers to craft interventions to manage these transactional environments. Applications beyond LRFSs space are likely to yield poignant discoveries as well.

Last, a number of immediate empirical extensions with these two organizational genuses are on the horizon that can provide richer understanding of the applied science issues and strengthen methods and conceptual frameworks. Primary among these is using 
structural equation modeling techniques as a validation (that is, Stage 3) methodology.

The use of structural equation modeling would allow me to capture strategic orientation as a common latent variable, instead of as distinct, separable regression equations. Under this approach, strategic orientation could be modeled as an entity itself using the strategic reliance variables I investigate here for product and channel space. This approach would allow me to investigate causal chaining and address a conceptual matter inherent in this dissertation's work: I treat product and channel strategies as separable. The empirical evidence I find here indicates a clear, important link between product strategy and channel strategy that should be rectified through more advanced empirical approaches such as structural equation modeling. Through a structural model, I can account for simultaneity of strategic choices of the firm and investigate the fit of alternate causal specifications to determine which model best fits the observed data, thus providing insight as to the relationship between the two strategic realms. This provides an alternative — and perhaps more robust — means of validating the natural kinds in the final postulated clustering solution as a set of species.

\subsubsection{Enabling an Ecological Approach: Use of the OSC for Modeling Econosystems and Implications for Organizational Evolution Theory}

The developments I make in this dissertation —including the extensions discussed earlier-have implications for two critical elements of inquiry. The first implication is for our ability to model the complex interactions within econosystems and their outcomes. There are a multiplicity of research questions and new methodological applications that may arise from having a cogent understanding of distinct organizational forms within, for example, LRFSs. This includes knowledge of their relative populations, their behaviors in relation to other forms, their use of resources and their reactions to environmental 
perturbations. This is an inherently ecological approach to understanding market structures across supply-value chains.

The information obtained from classificatory work and the identification of organizational species can be used to model these functional roles and the value created through interactions of species using empirical tools such as social network analysis and agent-based modeling. Applications of social network analysis tools can help identify the underlying structure of the econosystem, including the affective or cognitive ties that bind the network, the flow of informational resources that can be used by firms to form successful strategies, and the transmission of value of different exchange relationships. An agent-based modeling approach allows us to create simulations to understand how interventions or other environmental changes may affect system outcomes. For example, what happens if a FHO of a given species or organizational form is created within the system? Or what kind of capacity growth would be needed to respond to an environmental shock such as, say, the occurrence of a major pandemic that disrupts conventional supply chains? Unlike the natural sciences, in the social sciences our ability to engage in experiments with controls and treatments is limited. This means that we must find ways to analyze "natural experiments" and observational research, as well as to perform simulations. These tools provide approximations and expectations and allow us to make inferences about the range and magnitude of effects.

The second implication revolves around how my classificatory work can inform the theory of organizational evolution. As I note in my earlier chapters, extant theories of change within organizational populations are currently based on problematic empirics. Most of these theories have been propelled by work that does not disengage the 
delimitation of population boundaries from the theorized processes of change. This does not mean we should outright reject these theories; they may have elements of truth. But it raises questions about the validity of inferences that have been drawn about their universality. Are we truly to believe claims that a selective process based in legitimation is the likely causal mechanism of change within an organizational population when legitimation is itself used as the means to define the boundary of the case set? Other works are predicated on the use of artificial—and not emergent—categories or methods of classification and thus may fail to capture distinct processes of change because the category is overly broad. Thus, theories of change must be empirically validated using a viewpoint neutral framework, such as the OSC, as the basis for identifying organizational populations.

The need for separability of category identification and choosing the story of origins is a key aspect of McKelvey's often ignored work on developing a true organizational systematic — of creating an "organizational tree of life." My OSC approach addresses this call for separability. Use of the OSC allows us not only to test theories of origins but also to have a separate debate about how we place species in relation to one another: cladistics versus phylogenetics. Many adopt a phylogenetic approach; McKelvey does, as do Nelson and Winter implicitly. However, I am not yet convinced that this is the appropriate way to construct the Tree of Organizational Life; phyllogenetics presumes some form of linear "gene" transmission, which implies not only that organizations have "genetic material" but that theories of change for organizations are wholly selective. Consider as a counterfactual that in biology there are models of horizontal gene transmission that might allow us to continue in allegory adapted to the 
reality we observe in organizational space. This still ignores that exploring "genetic material" for organizations is treacherous. Moreover, phylogenetic approaches to taxonomic hierarchies make it difficult to account for the astute observation by Hanna, Carrol, and Pólos that organizational forms are not necessarily fixed in a chronological space - populations of organizations do not go extinct, unable to be resurrected from the ashes of time. They do not fossilize. As social objects, an organizational form may fall out of favor only to return again decades or centuries later. This is at least possible theoretically (although I know of no empirical evidence showing cases of this occurring).

Another important question along similar lines is raised by this work: not only must we consider the metaphysics at play when determining how we construct the origin story that orders organizational forms in relation to one another, we must also address the hegemony of Universal Darwinism that has pervaded the theoretical landscape which attempts to explain the mechanisms of change. Selection-based models of organizational evolution have come to be predominant under the population ecology approach. My observations in my work on classification and burgeoning extensions indicate that a more expansive set of evolutionary theories must be explored. This has been the case in biology and is the case now. Among these is the community ecology approach, which allows for the emergence of organizational forms through adaptive mechanisms, as well as selective ones.

In my study of the nascent population of FHOs, I find that constitutive and nonconstitutive attributes of the firms matter. In the results I present, many attributes of FHOs are shared with other organizational populations that serve LRFSs — such as farmers markets and community-supported agriculture programs — which tie consumers 
directly to local producers. Qualitative research done on background indicates that some of the extant cases among the population of FHOs have "evolved" from such farmers markets and community-supported agriculture programs. Likewise, this qualitative evidence indicates that some cases of FHOs were once operations in conventional distribution that engaged in strategic repositioning to enter the opportunity space created by the local foods movement. These and other de novo food hubs have mimicked attributes, seeing them as essential elements of the HPCs which carry meaning for other organizations in farm-to-table markets. Furthermore, triangulation of the forthcoming classificatory investigation of primary agricultural producers with findings here also provides an early signal that at least one of the FHO species identified in this work may be established as a community response in specific environments and/or by specific demographics of primary agricultural producer firms (those with socially disadvantaged primary operators).

The theorized stories that come from such observations indicate that it is not only environmental selection driving organizational form; these appear to be cases of adaptive processes such as migration or niche construction. The present study cannot elucidate this clearly, providing ample room for continued research on the behaviors that lead to organizational species. These behaviors are similar to the more recently identified processes underlying biological speciation, such as migration. Understanding those behaviors does not, however, explicitly identify the mechanisms - the causal drivers of a shift in homeostatic properties from one cluster or patterns to another. That is the purview of evolutionary research itself, and thus my observations here encourage testing evolutionary theory and forming new theories to be tested. 


\subsection{Contributions}

The work I present in this dissertation contributes to foundational scientific ideas and to applied knowledge about the social world. It contributes to fundamental science in the development of the OSC, a means of classifying organizational forms into distinct populations. This includes providing a set of methodological procedures to operationalize the OSC for initial identification of kinds and their validation as species. The concept I propose mitigates the issues that have plagued organizational systematics for the past 40 or more years. This includes the first such method that has substantial tractability for use in the organizational sciences and social scientific enquiry writ large; all previous models have had significant philosophical errors, lacked a distinct method of operationalization, and/or were vexed by inaccessible constructs.

In support of the OSC's development, my work also contributes to the philosophy of social science and the metaphysics of social objects. This contribution is contained within the philosophical model of Chapter 3, which integrates the work of Searle, Epstein, and Boyd. That model provides a useful avenue for classification of social objects generally, applied here to classify organizations specifically. Building upon the work of the notable philosophers it draws on, my integrated model is designed with the practice of social science in mind. Thus, it addresses a major criticism of at least one of those philosopher colleagues.

This dissertation's contribution to the fundamental practice of science is the conceptual framework outlined in Chapter 2 that can be used to identify the boundaries of a social phenomena such as the local foods movement. Of note is that although I apply that framework here to the local foods movement, the framework can generally be 
applied to other social scientific phenomena in which a change in societal attitudes and beliefs (a social movement) establishes new institutional arrangements and behaviors and creates a new opportunity space in the socioeconomic world. Its application to other social movements will not necessarily yield the same outcomes as it did for the local foods movement, given that the institutions and arrangements that arise to meet the imaginary (the combined vision formed by the new attitudes and beliefs) may be holistically different that they are for the local foods movement. In my application of the framework, I relied on a review of the literature approach given that the body of scientific literature on the social movement for which I sought to define boundaries is well advanced. However, original empirical research can also elucidate needed information for other applications of the framework where the body of scientific literature is still nascent. I contribute to applied science- - specifically that related to the policy objective of scaling-up local and regional food systems - by applying the OSC to a sample of the population of food hub organizations in the United States. This application enables an ecological approach to the scaling-up challenge, identifying the different roles played by FHO species in the networks of agents that form LRFSs. In the background of this application are the boundaries of the local food movement determined through use of Chapter 2's framework, as well as additional guidance from the literature on the organizational economics of FHOs. Through this work, I provide a proof of concept for the OSC, and, more important for the practice of applied social science, this proof of concept yields information useful to designing and implementing policy interventions that seek to address the scaling-up challenge through FHOs. 
Far more can be done to advance the foundational and applied science research streams I initiate in this dissertation. In this chapter, I identified many of those, as well as limitations that can be overcome in future entries in the applied portion of that work. Some of that work has already begun, some of it is near completion, not only advancing our understanding of organizational classification but assisting us as social scientists in guiding the leaders of our society with objective information they can (hopefully) deploy to advance objectives that create sustainable livelihoods for the producers, consumers, and every stage in agrofood systems. 


\section{REFERENCES}

Abatekassa, G., and Peterson, H. C. 2011. Market Access for Local Food through the Conventional Food Supply Chain. International Food and Agribusiness Management Review, 14(1), 41-60.

Aggestam, V., Fleiß, E., and Posch, A. 2017. Scaling-up short food supply chains? A survey study on the drivers behind the intention of food producers. Journal of Rural Studies, 51, 64-72.

Albert, S., and D.A. Whetten. 1985. Organizational Identity. Vol. 7, in Research on Organizational Behavior, edited by B. Staw and L.L. Cummings, 263-295. Greenwich, CT: JAI Press.

Asher, C. C., Mahoney, J. M., and Mahoney, J. T. 2005. Towards a Property Rights Foundation for a Stakeholder Theory of the Firm. Journal of Management and Governance, 5-32.

Ashforth, B.E., and F. Mael. 1989. "Social identity theory and the organization." Academy of Management Review 14 (1): 20-39.

Ashforth, B.E., and F.A. Mael. 1996. "Organizational identity and strategy as a context for the individual." Advances in Strategic Management 19 - 64.

Astley, W. Graham, and Andre H. Van de Ven. 1983. "Central Perspectives and Debates in Organization Theory." Administrative Science Quarterly 28 (2): 245-273.

Astley, W. Graham. 1985. "The Two Ecologies: Population and Community Perspectives on Organizational Evolution." Administrative Science Quarterly 30 (2): 224-241.

Bailey, Kenneth D. 1994. Typologies and Taxonomies: An Introduction to Classification Techniques. Vol. in the Series: Quantitative Applications in the Social Sciences. Thousand Oaks, CA: Sage University Paper.

Barbera, F., and Dagnes, J. 2016. Building Alternatives from the Bottom-up: The Case of Alternative Food Networks. Agriculture and Agricultural Science Procedia, $324-331$.

Barham, E. 1997. Social movements for sustainable agriculture in France: A polanyian perspective. Society and Natural Resources, 239-249.

Barham, J. 2011. Regional Food Hubs: Understanding the scope and scale of food hub operations. Washington, District of Columbia: USDA Agricultural Marketing Service. 
Barham, J., Tropp, D., Enterline, K., Farbman, J., Fisk, J., and Kiraly, S. 2012. Regional Food Hub Resource Guide. Washington, D.C.: USDA Agricultural Marketing Service.

Barney, J., and R. E. Hoskisson. 1990. "Strategic groups: Untested assertions and research proposals." Managerial and Decision Economics 187-198.

Baum, J. A., and Singh, J. V. 1996. Dynamics of Organizational Responses to Competition. Social Forces, 74(4), 1261-1297.

Beckie, M. A., Kennedy, E. H., and Wittman, H. 2012. Scaling up alternative food networks: farmers' markets and the role of clustering in western Canada. Agriculture and Human Values, 29, 333-345.

Behling, Orlando. 1980. "The Case for the Natural Science Model for Research in Organizational Behavior and Organization Theory." The Academy of Management Review, 483-490.

Berry, S., J. Levinson, and A. Pakes. 1995. "Automobile Prices in Market Equilibrium." Econometrica 841-890.

Berti, G., and Mulligan, C. 2016. Competitiveness of Small Farms and Innovative Food Supply Chains: The Role of Food Hubs in Creating Sustainable Regional and Local Food Systems. Sustainability, 8(616).

Beus, C. E., and Dunlap, R. E. 1990. Conventional Versus Alternative Agriculture: The Paradigmatic Roots of the Debate. Rural Sociology, 590-616.

Blay-Palmer, A., Landman, K., Knezevic, I., and Hayhurst, R. 2013. Constructing resilient, transformative communities through sustainable "food hubs". Local Environment, 521-528.

Bloom, Dara, J., and Hinrichs, C. C. 2011, March. Moving local food through conventional food system infrastructure: Value chain framework comparisons and insights. Renewable Agriculture and Food Systems, 26(1), 13-23.

Boeken, Bertrand, and Moshe Shachak. 2006. "Linking Community and Ecosystem Processes: The Role of Minor Species." Ecosystems 9: 119-127. doi:10.1007/s10021-004-0079-x.

Bogaert, Sandy, Christophe Boone, and Glenn R. Carroll. 2010. "Organizational Form Emergence and Competing Professional Schemata of Dutch Accounting, 1884 1939." In Categories in Markets: Origins and Evolution, by Greta Hsu, Giacomo Negro and Özgecan Koçak, 115-150. Emerald Group Publishing Limited. 
Boone, C., and Özcan, S. 2014. Why do cooperatives emerge in a world dominated by corporations? The diffusion of cooperatives in the U.S. bio-ethanol industry, 1978-2013. Academy of Management Journal, 990-1012.

Born, B., and Purcell, M. 2006. Avoiding the Local Trap: Scale and Food Systems in Planning Research. Journal of Planning Education and Research, 195-207.

Boyd, R. 1991. "Realism, Anti-foundationalism, and the Enthusiasm for Natural Kinds." Philosophical Studies 61: 127-148.

Boyd, R. 1999. "Homeostasis, species, and higher taxa." In Species: New interdisciplinary essays, edited by R. A. Wilson, 141-185. Cambridge, MA: MIT Press.

Boyd, R. 2003. " Finite beings, finite goods: The semantics, metaphysics and ethics of naturalist, Part I." Philosophy and Phenomenological Research 505-553.

Cangiani, M. 2011. Karl Polanyi’s institutional theory: Market society and its "disembedded" economy. Journal of Economic Issues, 45(1), 177-198.

Carper, William B., and William E. Snizek. 1980. "The Nature and Types of Organizational Taxonomies: An Overview." The Academy of Management Review 65-75.

Carroll, Glenn R. 1984. "Organizational Ecology." Annual Review of Sociology 71-93.

Carroll, Glenn R. 1985. "Concentration and specialization: dynamics of niche width in populations of organizations." American Journal of Sociology 90: 1262-1283.

Carroll, Glenn R., and A. Swaminathan. 2000. "Why the Microbrewery Movement? Organizational Dynamics of Resource Partitioning in the US Brewing Industry." American Journal of Sociology 715-762.

Carroll, Glenn R., and M.T. Hannan. 2000. The Demography of Corporations and Industries. Princeton, NJ: Princeton University Press.

Carroll, Glenn R., Michael T. Hannan, and László Pólos. 2000. "Forms and Populations." In The Demography of Corporations and Industries, by Glenn R. Carroll and Michael T. Hannan, 59 - 84. Princeton, NJ: Princeton University Press.

Cattani, G., J. Porac, and H. Thomas. 2017. "Categories and Competition." Strategic Management Journal 38 (1): 64-92.

Caves, R.E., and M.E. Porter. 1977. "From entry barriers to mobility barriers: conjectural decisions and contrived deterrence to new competition." Quarterly Journal of Economics 241-261. 
Chaddad, F. 2012. Advancing the theory of the cooperative organization: the cooperative as a true hybrid. Anals of Public and Cooperative Economics, 445461.

Chase, Jonathan M., and Mathew A. Leibold. 2003. Ecological Niches: Linking Classical and Contemporary Approaches. Chicago: University of Chicago Press.

Clancy, K., and Ruhf, K. 2010. Is Local Enough? Some Arguments for Regional Food Systems. Choices, 25(1).

Clarivate Analytics. 2020, January 17. Web of Science. Retrieved from http://www.webofknowledge.com

Clark, J. K., and Inwood, S. M. 2016. Scaling-up regional fruit and vegetable distribution: potential for adaptive change in the food system. Agriculture and Human Values, 33(3), 503-519.

Cleveland, D. A., Müller, N. M., Tranovich, A. C., and Mazaroli, D. N. 2014. Local food hubs for alternative food systems: A case study from Santa Barbara County, California. Journal of Rural Studies, 26-36.

Coff, R. W. 1999. When Competitive Advantage Doesn't Lead to Performance: The Resource-Based View and Stakeholder Bargaining Power. Organization Science, 119-133.

Coit, M. 2008. Jumping on the next bandwagon: An overview of the policy and legal aspects of the local food movement. Journal of Food Law and Policy, 45-70.

Colwell, R. K. 1994. "Niche: A Bifurcation in the Conceptual Lineage of the Term." In Keywords in Evolutionary Biology, edited by Keywords in Evolutionary Biology, 241-248. Cambridge, MA: Harvard University Press.

Connelly, S. 2010, November 20. Scaling Up Local Food. i4. Retrieved from communityrenewal.ca/renouveaucommunautaire.ca

Cook, M., Chaddad, F., and Iliopoulos, C. 2003. Advances in Cooperative Theory Since 1990: A Review of the Agricultural Economics Literature. In G. Hendrikse (Ed.), Restructuring Agricultural Cooperatives (pp. 65-90). Haveka.

Corley, K.G., C.V. Harquail, M.G. Pratt, M.A. Glynn, M. Fiol, and M.J. and Hatch. 2006. "Guiding identity theory through aged adolescence." Journal of Management Inquiry 85-99.

Darby, K., Batte, M. T., Ernst, S., and Roe, B. 2008. Decomposing Local: A Conjoint Analysis of Local Produced Foods. American Journal of Agricultural Economics, 476-486. 
Demil, B., and Lecocq, X. 2006. Neither Market nor Hierarchy nor Network: The Emergence of Bazaar Governance. Organization Studies, 1447-1466.

Diamond, A., and Barham, J. 2012. Moving Food Along the Value Chain: Innovation in Regional Food Distribution. Washington, D.C.: USDA Agricultural Marketing Service.

Diamond, A., Tropp, D., Barham, J., Muldoon, M. F., Kiraly, S., and Cantrell, P. 2014. Food Value Chains: Creating Shared Value to Enhance Marketing Success. Washington, DC: U.S. Dept. of Agriculture, Agricultural Marketing Service. Retrieved from http://dx.doi.org/10.9752/MS141.05-2014

DiMaggio, P. 1986. " Structural analysis of organizational fields: A blockmodel approach." Research in Organizational Behavior 335-370.

Dobrev, S.D., T.Y. Kim, and G.R. Carroll. 2002. "Dynamics of niche width and resource partitioning." American Journal of Sociology 106: 1299-1337.

Dolnicar, Sara. 2002. "A Review of Unquestioned Standards in Using Cluster Analysis for Data-driven Market Segmentation." Conference Proceedings of the Australian and New Zealand Marketing Academy Conference. Melbourne.

Dréo, J. (n.d.). Sustainable Development. Available via: https://en.wikipedia.org/wiki/File:Sustainable_development.svg. Wikimedia Commons, License: https://creativecommons.org/licenses/by-sa/3.0/deed.en.

DuPuis, E. M., and Goodman, D. 2005. Should we go "home" to eat?: toward a reflexive politics of localism. Journal of Rural Studies, 359-371.

Elton, Charles. 1927. Animal Ecology. London: Sidgwick and Jackson.

Epstein, B. 2019. "What are social groups? Their metaphysics and how to classify them." Synthèse 4899 - 4932.

Epstein, Brian. 2015. The Ant Trap: Rebuilding the Foundations of the Social Sciences. New York: Oxford University Press.

Epstein, Brian. 2016. "Précis of The Ant Trap: Rebuilding the Foundations of the Social Sciences." Journal of Social Ontology 2 (1): 125-134. doi:10.1515/jso2016-0001.

Ereshefsky, M., and T.A.C. Reydon. 2015. "Scientific Kinds." Philosophical Studies 969-986.

Eriksen, S. N. 2013. Defining local food: constructing a new taxonomy - three domains of proximity. Acta Agriculturae Scandinavica, Section B - Soil and Plant Science, 47-55. 
Ernest, S. K. Morgan, and James H Brown. 2001. "Homeostasis and Compensation: The Role of Species and Resources in Ecosystem Stability." Ecology 82 (8): 2118-2132.

Feagan, R. 2007. The place of food: mapping out the 'local' in food systems. Progress in Human Geography, 23-42.

Feagan, R. B., and Morris, D. 2009. Consumer quest for embeddedness: a case study of the Brantford Farmers' Market. International Journal of Consumer Studies, 235-243.

Fischer, M., Pirog, R., and Hamm, M. W. 2015. Food Hubs: Definitions, Expectations, and Realities. Journal of Hunger and Environmental Nutrition, 92-99.

Fligstein, N. 2001. The Architecture of Markets: An Economic Sociology of TwentyFirst-Century Capitalist Societies. Princeton, N.J.: Princeton University Press.

Foreman, P.O., and Westgren, R. E. 2019. The Dynamics of Collective Identity: Legitimacy, Identification and Commitment in Collectives. Academy of Management Annual Meeting Proceedings. Boston, Mass.

Foreman, P.O., Westgren, R. E., and Whetten, D. L. 2013. Creating Shared Identities in Collective Entrepreneurship: The Process of Identity Construction in Emergent Organizational Collectives. Academy of Management Annual Meeting Proceedings. Academy of Management.

Freeman, J., and M.T. Hannan. 1983. "Niche width and the dynamics of organizational populations." American Journal of Sociology 88: 1116-1145.

Fridman, J., and Lenters, L. 2013. Kitchen as food hub: adaptive food systems governance in the City of Toronto. Local Environment, 543-556.

Friedmann, H. 2007. Scaling up: Bringing public institutions and food service corporations into the project for a local, sustainable food system in Ontario. Agriculture and Human Values, 24, 389-398.

Galt, R. E. 2017. Alternative food movement. The International Encyclopedia of Geography.

Goodman, D. 1999. Agro-food studies in the 'age of ecology': Nature, corporeality, bio-politics. Sociologia Ruralis, 29, 17-38.

Goodman, D. 2004. Rural Europe Redux? Reflections on Alternative Agro-Food Networks and Paradigm Change. Sociologia Ruralis, 3-16. 
Grando, S., Carey, J., Hegger, E., Jahrl, I., and Ortolani, L. 2017. Short Food Supply Chains in Urban Areas: Who Takes the Lead? Evidence from Three Cities across Europe. Urban Agriculture and Regional Food Systems.

Granovetter, M. 1985. Economic action and social structure: the problem of embeddedness. American Journal of Sociology, 91, 481-510.

Gray, T. W., and Stevenson, G. 2008. Cooperative Structure for the Middle: Mobilizing for Power and Identity. In T. A. Lyson, G. W. Stevenson, and R. Welsh (Eds.), Food and the Mid-level Farm: Renewing an Agriculture of the Middle (pp. 37 - 55). Cambridge, Mass.: The MIT Press.

Guptill, A., and Welsh, R. 2008. Is Relationship Marketing an Alternative to the Corporatization of Organics? A Case Study of OFARM. In T. A. Lyson, G. W. Stevenson, and R. Welsh (Eds.), Food and the Mid-level Farm: Renewing an Agriculture of the Middle (pp. 55 - 78). Cambridge, Mass.: The MIT Press.

Guptill, A., and Wilkins, J. L. 2002. Buying into the food system: Trends in food retailing in the US and implications for local foods. Agriculture and Human Values, 39-51.

Hannan, M. T., and Carroll, G. R. 1992. Dynamics of Organizational Populations: Density, Legitimation, and Competition. New York: Oxford University Press.

Hannan, Micahel T., and John Freeman. 1986. "Where do organizational forms come from?" Sociological Forum 50-72.

Hannan, Michael T., and John Freeman. 1977. "The Population Ecology of Organizations'." American Journal of Sociology 82 (5): 929 - 964.

Hannan, Michael T., and John Freeman. 1984. "Structural Inertia and Organizational Change." American Sociological Review 49 (2): 149-164.

Hannan, Michael T., Glenn R. Carroll, and László Pólos. 2003. "The Organizational Niche." Sociological Theory 309 - 340.

Hannan, Michael T., László Pólos, and Glenn R. Carroll. 2004. Toward a Theory of Forms: Similarity and Categorization. Authors, Working Paper. Dated April 2, 2004. https://www.researchgate.net/publication/265248074.

Hannan, Michael T., Lászlo Pólos, and Glenn R. Carroll. 2007. Logics of Organization Theory: Audiences, Codes, and Ecologies. Princeton, NJ: Princeton University Press.

Hardy, J., Hamm, M., Pirog, R., Fisk, J., Farbman, J., and Fischer, M. 2016. Findings of the 2015 National Food Hub Survey. East Lansing, MI: Michigan State 
University Center for Regional Food. Retrieved from

http://foodsystems.msu.edu/resources/2015-food-hub-survey

Harrigan, Kathryn Rudie. 1985. "An application of clustering for strategic group analysis." Strategic Management Journal 6 (1): 55-73.

Hempel, Carl G. 1965. Aspects of Scientific Explanation (And Other Essays in the Philosophy of Science). New York: The Free Press.

Hendrikse, G., and Bijman, J. 2002. Ownership Structure in Agrifood Chains: The Marketing Cooperative. American Journal of Agricultural Economics, 104-119.

Henning, Christian, Marina Meila, Fionn Murtagh, and Roberto Rocci. 2016.

Handbook of Cluster Analysis. Vol. Chapman and Hall/CRC Handbooks of Modern Statistical Methods. Boca Raton, FL: CRC Press of the Taylor and Francis Group.

Higgins, V., Dibden, J., and Cocklin, C. 2008. Building alternative agri-food networks: Certification, embeddedness and agri-environmental governance. Journal of Rural Studies, 15-27.

Hinrichs, C. C. 2000. Embeddedness and local food systems: notes on two types of direct agricultural market. Journal of Rural Studies, 295-303.

Hinrichs, C. C. 2003. The practice and politics of food system localization. Journal of Rural Studies, 33-45.

Hinrichs, C., Kloppenburg, J., Stevenson, S., Lezberg, S., Hendrickson, J., and DeMaster, a. K. 1998. Moving beyond global and local. United States Department of Agriculture. Retrieved from http://www.ces.ncsu.edu/depts/sociology/ne185/global.html

Horst, M., Ringstrom, E., Tyman, S., Ward, M. K., Werner, V., and Born, B. 2011. Toward a more expansive understanding of food hubs. Journal of Agriculture, Food Systems, and Community Development, 209-225.

Hutchinson, G. Evelyn. 1957. "Some Concluding Remarks." Cold Spring Harbor Symposium on Quantitative Biology. 415-427.

Hutchinson, G. Evelyn. 1975. The Ecological Theater and the Evolutionary Play. New Haven: Yale University Press.

Hutchinson, G. Evelyn. 1991. "Population Studies: Animal Ecology and Demography." Bulletin of Mathematical Biology 193-213. 
Ilbery, B., and Maye, D. 2005. Alternative (shorter) food supply chains and specialist livestock products in the Scottish - English borders. Environment and Planning A, 37, $823-844$.

Ilbery, B., and Maye, D. 2005. Food supply chains and sustainability: evidence from specialist food producers in the Scottish/English borders. Land Use policy, 331 $-344$.

Johnson, R., Aussenberg, R. A., and Cowan, T. 2013. The Role of Local Food Systems in U.S. Farm Policy. Washington, DC: Congressional Research Service of the United States.

Kantarelis, Demetrius. 2017. Theories of the Firm. 5th. Inderscience.

Kelty, Matthew J. 2006. "The role of species mixtures in plantation forestry." Forest Ecology and Management 233: 195-204.

Ketchen, D. J., and C. L. Shook. 1996. "The application of cluster analysis in strategic management research: an analysis and critique." Strategic Management Journal 441-458.

King, R. P., Hand, M. S., DiGiacomo, G., Clancy, K., Gomez, M. I., Hardesty, S. D., . . . McLaughlin, E. W. 2010. Comparing the Structure, Size, and Performance of Local and Mainstream Food Supply Chains. Washington, DC: U.S. Department of Agriculture, Economic Research Service.

Klein, K., and Michas, A. 2014. The Farm Fresh Healthcare Project: Analysis of a Hybrid Values-based Supply Chain. Journal of Agriculture, Food Systems, and Community Development.

Knox, Eric B. 1998. "The use of hierarchies as organizational models in systematics." Biological Journal of the Linnean Society 1-49.

Laforge, J. M., Anderson, C. R., and McLachlan, S. M. 2017. Governments, grassroots, and the struggle for local food systems: containing, coopting, contesting and collaborating. Agriculture and Human Values, 663-681.

Lamine, C., Renting, H., Rossi, A., Wiskerke, J., and Brunori, G. 2012. Agri-Food systems and territorial development: innovations, new dynamics and changing governance mechanisms. In I. Darnhofer, D. Gibbon, and B. Dedieu (Eds.), Farming Systems Research 229 (pp. 229-256). Dordrecht: Springer Science+Business Media.

Lawton, John H. 1994. "What Do Species Do in Ecosystems?" Oikos 71 (3): 367-374.

LeBlanc, J. R., Conner, D., McRae, G., and Darby, H. 2014. Building resilience in nonprofit food hubs. Journal of Agriculture, Food Systems, and Community 
Development, Advance online publication. Retrieved from http://dx.doi.org/10.5304/jafscd.2014.043.005

Lee, R. 2000. Shelter from the storm? Geographies of regard in the worlds of horticultural consumption and production. Geoforum, 31, 137-157.

Lerman, T., Feenstra, G., and Visher, D. 2012. A Practitioner's Guide to Resources and Publications on Food Hubs and Values-Based Supply Chains: A Literature Review. Agricultural Sustainability Institute, Sustainable Agriculture Research and Education Program. Davis, CA: University of California, Davis.

Levins, Richard, and Richard C. Lewontin. 1985. The Dialectical Biologist. Cambridge, MA: Harvard University Press.

Lewontin, Richard C. 2002. The Triple Helix: Gene, Organism, and Environment. Cambridge, MA: Harvard University Press.

Lockie, S., and Kitto, S. 2000. Beyond the Farm Gate: Production-Consumption Networks and Agri-Food Research. Sociologia Ruralis, 40(1), 3-19.

Low, S. A., Adalja, A., Beaulieu, E., Key, N., Martinez, S., Melton, A., . . Jablonski, B. B. 2015. Trends in U.S. Local and Regional Food Systems. Washington, D.C.: USDA Economic Research Service.

Low, S. A., and Vogel, S. 2011. Direct and Intermediated Marketing of Local Foods in the United States. Washington, D.C.: USDA Economic Research Service.

Lowndes, V., and Skelcher, C. 1998. The Dynamics of Multi-organizational Partnerships: An Analysis of Changing Modes of Governance. Public Administration, 313-333.

Lund, M. 2012. Multi-stakeholder Co-operatives: Engines of Innovation for Building a Healthier Local Food System and a Healthier Economy. Journal of Cooperative Studies, 32-45.

Lyson, T. A., Stevenson, G. W., and Welsh, R. (Eds.). 2008. Food and the Mid-Level Farm : Renewing and Agriculture of the Middle. Cambridge, Mass.: The MIT Press.

Madhok, A. 2002. Reassing the fundamental and beyond: Ronald Coase, the transaction cost and resource-based theories of the firm and the institutional structure of production. Strategic Management Journal, 232-550.

Mahoney, J. T. 2005. Economic Foundations of Strategy. Thousand Oaks, CA: Sage Publications, Inc. 
Marsden, T., Banks, J., and Bristow, G. 2000. Food Supply Chain Approaches: Exploring their Role in Rural Development. Sociologia Ruralis, 424-438.

Martinez, S., Hand, M., Pra, M. D., Pollack, S., Ralston, K., Smith, T., .. . Newman, C. 2010. Local Food Systems. Washington, D.C.: USDA Economic Research Service.

Matson, J., and Thayer, J. 2013, July 12. The role of food hubs in food supply chains. Journal of Agriculture, Food Systems, and Community Development, 3(4), 4347.

Matson, J., Shaw, J., and Thayer, J. 2014, January/February. Food hubs: an evolution of the co-op business model. Rural Cooperatives, pp. 4-7, 35.

Mayr, E. 1969. Principles of systematic zoology. New York: McGraw-Hill.

McCarthy, Ian Paul. 2005. "Toward a Phylogenetic Reconstruction of Organizational Life." Journal of Bioeconomics 271-307.

McCarthy, Ian. 1995. "Manufacturing classification: Lessons from organizational systematics and biological taxonomy." Integrated Manufacturing Systems 3748.

McDermott, G. A., Corredoira, R. A., and Kruse, G. 2016. Public-Private Institutions as Catalysts of Upgrading in Emerging Market Societies. Academy of Management Journal, 1270-1296.

McKelvey, Bill. 1982. Organizational Systematics: Taxonomy, Evolution, Classification. Berkeley, CA: University of California Press.

McKelvey, Bill. 1978. "Organizational Systematics: Taxonomic Lessons from Biology." Management Sciences 1428-1440.

McPherson, J. M., and Ranger-Moore, J. R. 1991. Evolution on a Dancing Landscape: Organizations and Networks in Dynamic Blau Space. Social Forces, 70(1), 1942.

Ménard, C. 2004. The Economics of Hybrid Organizations. Journal of Institutional and Theoretical Economics, 1-32.

Morton, Craig, Jillian Anable, and John D. Nelson. 2017. "Consumer structure in the emerging market for electric vehicles: Identifying market segments using cluster analysis." International Journal of Sustainable Transportation 11 (6): 443-459.

Mouillot, David, David R. Bellwood, Christopher Baraloto, Jerome Chave, Rene Galzin, Mireille Harmelin-Vivien, Michel Kulbicki, et al. 2013. "Rare Species 
Support Vulnerable Functions in High-Diversity Ecosystems." PLoS Biology 11 (5): e1001569. doi:doi:10.1371/journal.pbio.1001569.

Mount, P. 2012. Growing local food: scale and local food systems governance. Agriculture and Human Values, 29(1), 107-121.

Murari, Andrea, Emmanuele Peluso, Francesco Cianfrani, Pasquale Gaudio, and Michele Lungaroni. 2019. "On the Use of Entropy to Improve Model Selection Criteria." Entropy 21 (394).

Murdoch, J. 2000. Networks - a new paradigm of rural development? Journal of Rural Studies, 407-419.

Murdoch, J., Marsden, T., and Banks, J. 2000. Quality, Nature, and Embeddedness: Some Theoretical Considerations in the Context of the Food Sector. Economic Geography, 107-125.

Navin, M. 2015. Scaling-Up Alternative Food Networks. Journal of Social Philosophy, $46(4), 434-448$.

Navis, C., and M. A. Glynn. 2010. "How new market categories emerge: Temporal dynamics of legitimacy, identity, and entrepreneurship in satellite radio." Administrative Science Quarterly 439-471.

Nost, E. 2014. Scaling-up local foods: Commodity practice in community supported agriculture (CSA). Journal of Rural Studies, 34, 152-160.

Nurse, G., Onozaka, Y., and Thilmany McFadden, D. 2012. Consumer Motivations and Buying Behavior: The Case of the Local Food Systems Movement. Journal of Food Products Marketing, 18(5), 385 - 396.

Odling-Smee, F. J., K. N. Laland, and and Feldman. 2003. Niche Construction: The Neglected Process in Evolution. Princeton, N.J.: Princeton University Press.

Offer, A. 1997. Between the gift and the market: the economy of regard. Economic History Review, L 3, 450-476.

Park, Robert E., Ernest W. Burgess, and Roderick D. McKenzie. 1925. The City. Chicago: University of Chicago Press.

Parker, Simon C. 2010. "A Predator-Prey Model of Knowledge Spillovers and Entrepreneurship." Strategic Entrepreneurship Journal 4: 307-322.

Péli, G., and Bruggeman, J. 2006. Networks embedded in n-dimensional space: The impact of dimensionality change. Social Networks, 28(4), 449-453. 
Pinchot, A. 2014. The Economics of Local Food Systems: A Literature Review of the Production, Distribution, and Consumption of Local Food. University of Minnesota Extension Center for Community Vitality.

Podolny, J. M., and Page, K. L. 1998. Network Forms of Organization. Annual Review of Sociology, 57-76.

Podolny, J.M., and Stuart, T.E. 1995. "A Role-Based Ecology of Technological Change." American Journal of Sociology 1224-1260.

Polanyi, K. 2001. The Great Transformation: The Political and Economic Origins of Our Time (2nd ed.). Beacon Press.

Pólos, László, Michael T. Hannan, and Glenn R. Carroll. 1998. "Form and Identity: On the Structure of Organizational Forms." 14th Colloquium of the European Group on Organization Studies.

Pólos, László, Michael T. Hannan, and Glenn R. Carroll. 2002. "Foundations of a theory of social forms." Industrial and Corporate Change 11 (1): 85 - 115.

Popielarz, Pamela A., and Zachary P. Neal. 2007. "The Niche as a Theoretical Tool." Annual Review of Sociology 33: 65-84.

Powell, W. W. 1990. Neither Market nor Hierarchy: Network Forms of Organization. Research in Organizational Behavior, 295-336.

Renting, H., and Wiskerke, H. 2010. New Emerging Roles for Public Institutions and Civil Society in the Promotion of Sustainable Local Agro-Food Systems. WS4.4 - Transitions towards sustainable agriculture: From farmers to agrofood systems, (pp. 1902-1912). Vienna, Austria.

Renting, H., Marsden, T. K., and Banks, J. 2003. Understanding alternative food networks: exploring the role of short food supply chains in rural development. Environment and Planning A, 393 - 411.

Rich, Philip. 1992. "The Organizational Taxonomy: Definition and Design." The Academy of Management Review 758-781.

Romanelli, Elaine. 1991. "The Evolution of New Organizational Forms." Annual Review of Sociology 79-103.

Ruef, M. 1997. Assessing organizational fitness on a dynamic landscape: an empirical test of the relative inertia thesis. Strategic Management Journal, 18(11), 837853.

Sage, C. 2003. Social embeddedness and relations of regard: alternative 'good food' networks in south-west Ireland. Journal of Rural Studies, 47-60. 
Samuels, R., and M. Ferreira. 2010. "Why Don't Concepts Constitute a Natural Kind?" Behavioral and Brain Sciences 222-223.

Santini, F., and Gomez y Paloma, S. 2013. Short Food Supply Chains and Local Food Systems in the EU. A State of Play of their Socio-Economic Characteristics. Joint Research Centre, Institute for Prospective Technological Studies. Seville (Spain): European Commission.

Scott, R. 2013. Institutions and Organizations: Ideas, Interests, and Identities. 4th. SAGE Publications.

Searle, John R. 1995. The Construction of Social Reality. New York: The Free Press.

Searle, John R. 2010. Making The Social World : The Structure of Human Civilization. New York: Oxford University Press.

Simmel, G. 1955. The web of group-affiliations. In Conflict and the Web of Group Affiliations. Translated by R Bendix. New York.

Sonnino, R., and Marsden, T. 2006. Beyond the divide: rethinking relationships between alternative and conventional food networks in Europe. Journal of Economic Geography, 181-199.

Starr, A. 2010. Local Food : A Social Movement? Cultural Studies $<=>$ Critical Methodologies, 1-12.

Steinley, Douglas. 2019. "Lecture Notes for PSYCH 9765 - Cluster Analysis and Network Analysis." Columbia, MO: University of Missouri.

Stevenson, G., and Pirog, R. 2008. Values-Based Supply Chains: Strategies for Agrifood Enterprises of the Middle. In T. A. Lyson, G. Stevenson, and R. Welsh (Eds.), Food and the Mid-level Farm: Renewing an Agriculture of the Middle (pp. 119 - 147). Cambridge, Mass.: The MIT Press.

Stiglitz, Joseph E. 1991. "Symposium on Organizations and Economics." Journal of Economic Perspectives 5 (2): 15 - 24.

Stroink, M. L., and Nelson, C. H. 2013. Complexity and food hubs: five case studies from Northern Ontario. Local Environment, 620-635.

Swaminathan, A., and Delacroix, J. 1991. Differentiation Within An Organizational Population: Additional Evidence from The Wine Industry. Academy of Management Journal, 34(3), 679-692.

Swindler, J.K. 2016. "Brian Epstein. The Ant Trap: Rebuilding the Foundations of the Social Sciences. Oxford University Press 2015. 312 pp. \$39.95 USD 
(Hardcover ISBN 9780199381104)." Philosophy in Review XXXVI (3): 103 108.

Thilmany McFadden, D. 2015. What Do We Mean by "Local Foods"? Choices, 30(1).

Tirole, J. 1988. The Theory of Industrial Organization. Cambridge, MA: MIT Press.

Tregear, A. 2011. Progressing knowledge in alternative and local food networks: Critical reflections and a research agenda. Journal of Rural Studies, 419-430.

US Department of Agriculture Economic Research Service. 2019, August 20. Local and Regional Foods. Retrieved from Agriculture Improvement Act of 2018: Highlights and Implications: https://www.ers.usda.gov/agricultureimprovement-act-of-2018-highlights-and-implications/local-and-regionalfoods/

Uzzi, B. 1997. "Social structure and competition in interfirm networks: The paradox of embeddedness." Administrative Science Quarterly 35-67.

Venn, L., Kneafsey, M., Holloway, L., Cox, R., Dowler, E., and Tuomainen, H. 2006. Researching European 'alternative' food networks: some methodological considerations. Area, 248-258.

Wald, N., and Hill, D. P. 2016. 'Rescaling' alternative food systems: from food security to food sovereignty. Agriculture and Human Values, 203-213.

Wallace, Rodrick. 2015. An ecosystem approach to economic stabilization: Escaping the neoliberal wilderness. New York: Routledge.

Watts, D., Ilbery, B., and Maye, D. 2005. Making reconnections in agro-food geography: alternative systems of food provision. Progress in Human Geography, 22-40.

Weber, K., Heinze, K. L., and DeSoucey, M. 2008. Forage for Thought: Mobilizing Codes in the Movement for Grass-fed Meat and Dairy Products. Administrative Science Quarterly, 529-567.

Wezel, Filippo Carlo, and Arjen van Witteloostuijn. 2003. "The Ecology of NicheWidth Strategies: Generalists, polymorphists and specialists in the UK motorcycle industry from 1895 to 1993." Working Paper. doi:10.1.1.195.2730.

Wezel, Filippo Carlo, and Arjen van Witteloostuijn. 2006. "From Scooters to Choppers: Product Portfolio Change and Organizational Failure." Long Range Planning 39: 11-28.

Whatmore, S., Stassart, P., and Renting, H. 2003. What's alternative about alternative food networks? Environment and Planning A, 389 - 391. 
Whatmore, Sarah, Richard Munton, Jo Little, and Terry Marsden. 1987. "Towards a Typology of Farm Businesses in Contemporary British Agriculture." Sociologia Ruralis 21-37.

White, H.C. 2002. Markets from Networks: Socioeconomic Models of Production. Princeton, N.J.: Princeton University Press.

Williamson, O. 1991. Comparative Economic Organization: The Analysis of Discrete Structural Alternatives. Administrative Science Quarterly, 269-296.

Winter, M. 2003. Embeddedness, the new food economy and defensive localism. Journal of Rural Studies, 23-32.

Wiskerke, J. S. 2010. On Places Lost and Places Regained: Reflections on the Alternative Food Geography and Sustainable Regional Development. International Planning Studies, 14(4), 369 - 387.

Wittman, H., Beckie, M., and Hergesheimer, C. 2012. Linking Local Food Systems and the Social Economy? Future Roles for Farmers' Markets in Alberta and British Columbia. Rural Sociology, 77(1), 36-61.

Young, D. R. 2001. Organizational Identity in Nonprofit Organizations: Strategic and Structural Implications. Nonprofit Management and Leadership, 139-157. 


\section{APPENDIX}

Table A.1 Numerical Data for Species Mean Profiles: Morphological Attributes

\begin{tabular}{|c|c|c|c|c|c|c|c|c|c|}
\hline & \multicolumn{2}{|c|}{ Species } & 1 & 2 & 3 & 4 & 5 & 6 & Total \\
\hline \multicolumn{10}{|l|}{ Variable } \\
\hline \multirow{3}{*}{$\begin{array}{c}\text { Natural log } \\
\text { of total } \\
\text { number of } \\
\text { years in } \\
\text { business }\end{array}$} & \multicolumn{2}{|c|}{ Mean } & 1.35 & 0.99 & 1.33 & 2.07 & 1.84 & 1.81 & 1.49 \\
\hline & \multirow[b]{2}{*}{$\begin{array}{c}95 \% \\
\text { CI }\end{array}$} & LB & 1.00 & 0.41 & 0.85 & 0.92 & 1.18 & 1.18 & 1.28 \\
\hline & & UB & 1.70 & 1.58 & 1.80 & 3.22 & 2.49 & 2.43 & 1.71 \\
\hline \multirow{3}{*}{$\begin{array}{c}\text { Profit } \\
\text { motivated } \\
\text { legal status }\end{array}$} & \multicolumn{2}{|c|}{ Mean } & 2.22 & 1.36 & 1.95 & 1.89 & 1.88 & 1.83 & 1.92 \\
\hline & \multirow{2}{*}{$\begin{array}{c}95 \% \\
\text { CI }\end{array}$} & LB & 1.87 & 0.78 & 1.51 & 1.29 & 1.71 & 1.24 & 1.75 \\
\hline & & UB & 2.57 & 1.94 & 2.39 & 2.49 & 2.05 & 2.43 & 2.10 \\
\hline \multirow{3}{*}{$\begin{array}{l}\text { Cooperative } \\
\text { form legal } \\
\text { status }\end{array}$} & \multicolumn{2}{|c|}{ Mean } & 0.00 & 0.14 & 0.11 & 0.44 & 1.00 & 0.17 & 0.26 \\
\hline & \multirow{2}{*}{$\begin{array}{c}95 \% \\
\text { CI }\end{array}$} & LB & 0.00 & -0.07 & -0.05 & 0.04 & 1.00 & -0.08 & 0.18 \\
\hline & & UB & 0.00 & 0.35 & 0.26 & 0.85 & 1.00 & 0.41 & 0.35 \\
\hline \multirow{3}{*}{$\begin{array}{l}\text { Acts as } \\
\text { broker }\end{array}$} & \multicolumn{2}{|c|}{ Mean } & 1.28 & 1.64 & 1.63 & 2.33 & 1.82 & 1.08 & 1.55 \\
\hline & \multirow{2}{*}{$\begin{array}{c}95 \% \\
\text { CI }\end{array}$} & LB & 1.05 & 1.16 & 1.17 & 1.56 & 1.37 & 0.90 & 1.39 \\
\hline & & UB & 1.51 & 2.13 & 2.09 & 3.10 & 2.28 & 1.27 & 1.72 \\
\hline \multirow{3}{*}{$\begin{array}{c}\text { \% of revenue } \\
\text { attributed to } \\
\text { non-sales }\end{array}$} & \multicolumn{2}{|c|}{ Mean } & 0.14 & 0.13 & 0.36 & 0.44 & 0.12 & 0.13 & 0.20 \\
\hline & \multirow{2}{*}{$\begin{array}{c}95 \% \\
\text { CI }\end{array}$} & LB & 0.05 & 0.02 & 0.15 & 0.14 & 0.07 & 0.04 & 0.14 \\
\hline & & UB & 0.23 & 0.23 & 0.56 & 0.74 & 0.17 & 0.23 & 0.26 \\
\hline \multirow{3}{*}{$\begin{array}{c}\text { Total } \\
\text { warehouse } \\
\text { space in } \\
\text { square feet }\end{array}$} & \multicolumn{2}{|c|}{ Mean } & 3379.06 & 839.29 & 14478.95 & 20022.56 & 5758.00 & 21100.00 & 8992.90 \\
\hline & \multirow{2}{*}{$\begin{array}{c}95 \% \\
\text { CI }\end{array}$} & LB & 1116.46 & -24.43 & 1209.20 & -16748.45 & -682.64 & 3978.19 & 4698.70 \\
\hline & & UB & 5641.67 & 1703.00 & 27748.69 & 56793.56 & 12198.64 & 38221.81 & 13287.10 \\
\hline \multirow{3}{*}{$\begin{array}{l}\text { Number of } \\
\text { delivery } \\
\text { vehicles on- } \\
\text { hand }\end{array}$} & \multicolumn{2}{|c|}{ Mean } & 2.47 & 0.71 & 5.37 & 1.67 & 1.71 & 8.17 & 3.23 \\
\hline & $95 \%$ & LB & 1.74 & 0.29 & 0.90 & 0.39 & 0.57 & -0.09 & 1.98 \\
\hline & CI & UB & 3.20 & 1.13 & 9.84 & 2.94 & 2.84 & 16.43 & 4.49 \\
\hline Offer & $\mathbf{M e}$ & & 1.00 & 0.00 & 1.00 & 0.56 & 0.71 & 0.83 & 0.76 \\
\hline transport & $95 \%$ & LB & 1.00 & 0.00 & 1.00 & 0.15 & 0.46 & 0.59 & 0.67 \\
\hline $\begin{array}{l}\text { services to } \\
\text { producers }\end{array}$ & CI & UB & 1.00 & 0.00 & 1.00 & 0.96 & 0.95 & 1.08 & 0.84 \\
\hline Additional & Me & & 1.31 & 1.57 & 2.11 & 1.56 & 0.71 & 1.25 & 1.41 \\
\hline packaging & $95 \%$ & LB & 0.94 & 0.70 & 1.65 & 0.78 & 0.20 & 0.77 & 1.19 \\
\hline $\begin{array}{l}\text { invorvement } \\
\text { level }\end{array}$ & $\mathrm{CI}$ & UB & 1.68 & 2.44 & 2.56 & 2.33 & 1.21 & 1.73 & 1.63 \\
\hline & Mc & & 0.00 & 0.14 & 0.89 & 0.67 & 0.00 & 0.17 & 0.26 \\
\hline $\begin{array}{l}\text { Processing } \\
\text { facilities }\end{array}$ & $95 \%$ & LB & 0.00 & -0.07 & 0.74 & 0.28 & 0.00 & -0.08 & 0.18 \\
\hline & $\mathrm{CI}$ & UB & 0.00 & 0.35 & 1.05 & 1.05 & 0.00 & 0.41 & 0.35 \\
\hline On-line & $\mathbf{M}$ & & 0.56 & 0.64 & 0.68 & 0.33 & 0.65 & 0.67 & 0.60 \\
\hline ordering & $95 \%$ & LB & 0.38 & 0.36 & 0.45 & -0.05 & 0.39 & 0.35 & 0.51 \\
\hline system & CI & UB & 0.74 & 0.93 & 0.91 & 0.72 & 0.90 & 0.98 & 0.70 \\
\hline Rental space & Mc & & 0.00 & 0.00 & 0.11 & 1.00 & 0.00 & 1.00 & 0.22 \\
\hline for other & $95 \%$ & LB & 0.00 & 0.00 & -0.05 & 1.00 & 0.00 & 1.00 & 0.14 \\
\hline businesses & $\mathrm{CI}$ & UB & 0.00 & 0.00 & 0.26 & 1.00 & 0.00 & 1.00 & 0.31 \\
\hline & Mc & & 0.28 & 0.14 & 0.21 & 1.00 & 0.00 & 0.00 & 0.23 \\
\hline Retail space & $95 \%$ & LB & 0.12 & -0.07 & 0.01 & 1.00 & 0.00 & 0.00 & 0.15 \\
\hline & $\mathrm{CI}$ & UB & 0.45 & 0.35 & 0.41 & 1.00 & 0.00 & 0.00 & 0.32 \\
\hline Licensed & Mc & & 0.03 & 0.07 & 0.26 & 0.67 & 0.00 & $\mathbf{0 . 0 0}$ & 0.13 \\
\hline shared use & $95 \%$ & LB & -0.03 & -0.08 & 0.05 & 0.28 & 0.00 & 0.00 & 0.06 \\
\hline kitchen & CI & UB & 0.09 & 0.23 & 0.48 & 1.05 & 0.00 & 0.00 & 0.19 \\
\hline
\end{tabular}

Note: 1 = Average Joes; 2 = Small-scale Startups; 3 = Processors; $4=$ Community Service Providers; 5 = Coops; 6

$=$ Traditional Produce Warehousers. $\mathrm{CI}=$ confidence interval. $\mathrm{LB}=$ lower bound. $\mathrm{UB}=$ upper bound. 
Table A.2 Numerical Data for Species Mean Profiles: Product Reliance

\begin{tabular}{|c|c|c|c|c|c|c|c|c|c|}
\hline & \multicolumn{2}{|c|}{ Species } & 1 & 2 & 3 & 4 & 5 & 6 & Total \\
\hline \multicolumn{10}{|l|}{ Variable } \\
\hline \multirow{3}{*}{$\begin{array}{c}\text { Fresh } \\
\text { produce } \\
\text { and herbs }\end{array}$} & \multicolumn{2}{|c|}{ Mean } & 65.31 & 53.86 & 46.01 & 34.17 & 50.92 & 67.66 & 55.91 \\
\hline & \multirow{2}{*}{$\begin{array}{c}95 \% \\
\mathrm{CI}\end{array}$} & LB & 53.96 & 32.89 & 26.45 & 6.92 & 31.79 & 50.01 & 48.96 \\
\hline & & UB & 76.65 & 74.84 & 65.57 & 61.41 & 70.06 & 85.31 & 62.87 \\
\hline \multirow{3}{*}{$\begin{array}{l}\text { Processed } \\
\text { produce }\end{array}$} & \multicolumn{2}{|c|}{ Mean } & 1.72 & 2.88 & 12.84 & 8.33 & 2.38 & 1.43 & 4.50 \\
\hline & \multirow{2}{*}{$\begin{array}{c}95 \% \\
\mathrm{CI}\end{array}$} & LB & 0.67 & -1.02 & -2.45 & -3.37 & -1.15 & 0.08 & 1.49 \\
\hline & & UB & 2.77 & 6.78 & 28.14 & 20.03 & 5.90 & 2.78 & 7.50 \\
\hline \multirow{3}{*}{$\begin{array}{l}\text { Meat and } \\
\text { poultry }\end{array}$} & \multicolumn{2}{|c|}{ Mean } & 14.36 & 24.80 & 15.32 & 8.00 & 30.33 & 7.16 & 17.51 \\
\hline & \multirow{2}{*}{$\begin{array}{c}95 \% \\
\mathrm{CI}\end{array}$} & LB & 5.74 & 6.06 & 2.81 & -1.96 & 13.67 & 2.29 & 12.33 \\
\hline & & UB & 22.99 & 43.54 & 27.83 & 17.96 & 46.98 & 12.03 & 22.68 \\
\hline \multirow{3}{*}{ Fish } & \multicolumn{2}{|c|}{ Mean } & 0.42 & 0.57 & 0.45 & 0.17 & 0.00 & 1.57 & 0.50 \\
\hline & \multirow{2}{*}{$\begin{array}{c}95 \% \\
\text { CI } \\
\end{array}$} & LB & -0.04 & -0.21 & -0.26 & -0.26 & 0.00 & -1.40 & 0.11 \\
\hline & & UB & 0.88 & 1.35 & 1.15 & 0.60 & 0.00 & 4.55 & 0.89 \\
\hline \multirow{3}{*}{$\begin{array}{l}\text { Milk and } \\
\text { other dairy } \\
\text { products }\end{array}$} & \multicolumn{2}{|c|}{ Mean } & 6.34 & 2.39 & 2.91 & 17.00 & 2.75 & 7.43 & 5.28 \\
\hline & \multirow{2}{*}{$\begin{array}{c}95 \% \\
\mathrm{CI} \\
\end{array}$} & LB & 2.28 & 0.15 & 0.51 & -2.41 & 1.21 & 2.49 & 3.47 \\
\hline & & UB & 10.40 & 4.64 & 5.31 & 36.41 & 4.28 & 12.37 & 7.10 \\
\hline \multirow{3}{*}{ Eggs } & \multicolumn{2}{|c|}{ Mean } & 3.54 & 2.72 & 2.80 & 5.00 & 3.75 & 4.03 & 3.47 \\
\hline & \multirow{2}{*}{$\begin{array}{c}95 \% \\
\text { CI }\end{array}$} & LB & 1.54 & 0.66 & 0.99 & -1.64 & 1.52 & 0.36 & 2.52 \\
\hline & & UB & 5.53 & 4.78 & 4.62 & 11.64 & 5.99 & 7.71 & 4.42 \\
\hline \multirow{3}{*}{$\begin{array}{l}\text { Grains, } \\
\text { beans } \\
\text { and/or } \\
\text { flours }\end{array}$} & \multicolumn{2}{|c|}{ Mean } & 1.55 & 8.02 & 1.42 & 1.17 & 0.60 & 2.45 & 2.36 \\
\hline & \multirow{2}{*}{$\begin{array}{c}95 \% \\
\text { CI }\end{array}$} & LB & 0.73 & -7.33 & 0.09 & -0.38 & 0.05 & -0.38 & 0.32 \\
\hline & & UB & 2.37 & 23.37 & 2.75 & 2.71 & 1.15 & 5.28 & 4.40 \\
\hline \multirow{3}{*}{$\begin{array}{c}\text { Baked } \\
\text { goods/bread }\end{array}$} & \multicolumn{2}{|c|}{ Mean } & 1.30 & 1.85 & 1.68 & 1.25 & 1.84 & 0.17 & 1.40 \\
\hline & $95 \%$ & LB & 0.35 & 0.40 & -0.28 & -0.95 & 0.32 & -0.12 & 0.84 \\
\hline & $\mathrm{CI}$ & UB & 2.25 & 3.31 & 3.65 & 3.45 & 3.35 & 0.47 & 1.96 \\
\hline & Mc & & 0.39 & 0.19 & 0.30 & 1.17 & 0.72 & 0.04 & 0.41 \\
\hline Coffee/tea & $95 \%$ & LB & 0.04 & -0.01 & -0.15 & -0.98 & 0.04 & -0.03 & 0.20 \\
\hline & CI & UB & 0.73 & 0.40 & 0.75 & 3.31 & 1.41 & 0.12 & 0.61 \\
\hline & Me & & 3.19 & 1.10 & 9.89 & 2.92 & 1.69 & 5.39 & 4.17 \\
\hline processed & & LB & -0.56 & -0.17 & -1.33 & -0.96 & 0.28 & -2.49 & 1.64 \\
\hline $\begin{array}{l}\text { or value- } \\
\text { added food } \\
\text { products }\end{array}$ & $\begin{array}{c}95 \% \\
\text { CI }\end{array}$ & UB & 6.94 & 2.37 & 21.12 & 6.79 & 3.11 & 13.28 & 6.70 \\
\hline & Mc & & 0.77 & 0.17 & 0.11 & 0.83 & 0.82 & 0.05 & 0.48 \\
\hline $\begin{array}{l}\text { Non-Iood } \\
\text { items }\end{array}$ & $95 \%$ & LB & 0.03 & -0.13 & -0.12 & -1.31 & 0.18 & -0.06 & 0.21 \\
\hline & $\mathrm{CI}$ & UB & 1.51 & 0.48 & 0.33 & 2.98 & 1.46 & 0.17 & 0.76 \\
\hline Other All & Mc & & 0.90 & 1.43 & 1.00 & 3.33 & 4.13 & 2.60 & 1.90 \\
\hline unclassified & $95 \%$ & LB & 0.07 & -1.49 & -0.61 & -5.24 & 0.51 & -2.25 & 0.87 \\
\hline & $\mathrm{CI}$ & UB & 1.72 & 4.35 & 2.61 & 11.90 & 7.74 & 7.46 & 2.93 \\
\hline
\end{tabular}

Note: Species codes $-1=$ Average Joes; $2=$ Small-scale Startups; 3 = Processors; 4= Community Service Providers; 5

$=$ Coops; $6=$ Traditional Produce Warehousers. $\mathrm{CI}=$ confidence interval. $\mathrm{LB}=$ lower bound. UB $=$ upper bound. 
Table A.3 Numerical Data For Species Mean Profiles: Channel Reliance

\begin{tabular}{|c|c|c|c|c|c|c|c|c|c|}
\hline & \multicolumn{2}{|c|}{ Species } & 1 & 2 & 3 & 4 & 5 & 6 & Total \\
\hline \multicolumn{10}{|l|}{ Variable } \\
\hline \multirow{3}{*}{$\begin{array}{l}\text { DTC Total } \\
\text { Percent of } \\
\text { Sales to } \\
\text { Consumers }\end{array}$} & \multicolumn{2}{|c|}{ Mean } & 28.98 & 64.14 & 49.04 & 45.13 & 46.34 & 37.28 & 42.90 \\
\hline & \multirow{2}{*}{$\begin{array}{c}95 \% \\
\text { CI }\end{array}$} & LB & 14.47 & 39.91 & 26.80 & -2.72 & 23.29 & 13.48 & 34.40 \\
\hline & & UB & 43.48 & 88.37 & 71.27 & 92.98 & 69.40 & 61.07 & 51.40 \\
\hline \multirow{3}{*}{$\begin{array}{c}\text { DTR Total } \\
\text { Percent of } \\
\text { Sales to Retail }\end{array}$} & \multicolumn{2}{|c|}{ Mean } & 52.98 & 20.05 & 17.95 & 29.40 & 39.42 & 50.57 & 37.72 \\
\hline & \multirow{2}{*}{$\begin{array}{c}95 \% \\
\text { CI }\end{array}$} & LB & 38.07 & 5.59 & 2.39 & 2.36 & 19.24 & 27.32 & 30.15 \\
\hline & & UB & 67.89 & 34.51 & 33.51 & 56.44 & 59.60 & 73.81 & 45.30 \\
\hline \multirow{3}{*}{$\begin{array}{l}\text { DTM Total } \\
\text { Percent of } \\
\text { Sales to } \\
\text { Intermediaries }\end{array}$} & \multicolumn{2}{|c|}{ Mean } & 4.34 & 5.71 & 11.01 & 8.00 & 13.58 & 0.94 & 7.19 \\
\hline & \multirow{2}{*}{$\begin{array}{c}95 \% \\
\text { CI }\end{array}$} & LB & 0.80 & -6.63 & -1.64 & $\begin{array}{r}- \\
14.21 \\
\end{array}$ & -0.45 & 0.08 & 3.33 \\
\hline & & UB & 7.89 & 18.06 & 23.67 & 30.21 & 27.61 & 1.80 & 11.04 \\
\hline \multirow{3}{*}{$\begin{array}{l}\text { DTI Total } \\
\text { Percent of } \\
\text { Sales to } \\
\text { Institutions }\end{array}$} & \multicolumn{2}{|c|}{ Mean } & 13.35 & 9.85 & 18.94 & 17.47 & 0.64 & 9.73 & 11.30 \\
\hline & \multirow{2}{*}{$\begin{array}{c}95 \% \\
\text { CI }\end{array}$} & LB & 4.91 & -3.77 & 3.66 & 26.02 & -0.06 & 2.65 & 6.80 \\
\hline & & UB & 21.80 & 23.47 & 34.22 & 60.96 & 1.34 & 16.80 & 15.79 \\
\hline \multirow{3}{*}{$\begin{array}{l}\text { Total Percent } \\
\text { of Sales to } \\
\text { Unclassified }\end{array}$} & \multicolumn{2}{|c|}{ Mean } & 0.35 & 0.25 & 3.06 & 0.00 & 0.01 & 0.98 & 0.82 \\
\hline & \multirow{2}{*}{$\begin{array}{c}95 \% \\
\text { CI }\end{array}$} & LB & -0.03 & -0.29 & -1.51 & 0.00 & -0.01 & -0.28 & 0.01 \\
\hline & & UB & 0.73 & 0.78 & 7.63 & 0.00 & 0.02 & 2.23 & 1.63 \\
\hline
\end{tabular}

Note: Species codes $-1=$ Average Joes; $2=$ Small-scale Startups; 3 = Processors; $4=$ Community Service Providers; 5

$=$ Coops $6=$ Traditional Produce Warehousers. $\mathrm{CI}=$ confidence interval. $\mathrm{LB}=$ lower bound. $\mathrm{UB}=$ upper bound. 
Table A.4 Numerical Data for Species Mean Profiles: Community Services

\begin{tabular}{|c|c|c|c|c|c|c|c|c|c|}
\hline & \multicolumn{2}{|c|}{ Species } & 1 & 2 & 3 & 4 & 5 & 6 & Total \\
\hline \multicolumn{10}{|l|}{ Variable } \\
\hline \multirow{3}{*}{$\begin{array}{l}\text { Paid employment } \\
\text { opportunities for youth }\end{array}$} & \multicolumn{2}{|c|}{ Mean } & 0.28 & 0.00 & 0.58 & 0.44 & 0.24 & 0.58 & 0.34 \\
\hline & \multirow{2}{*}{$\begin{array}{c}95 \% \\
\text { CI } \\
\end{array}$} & LB & 0.12 & 0.00 & 0.33 & 0.04 & 0.01 & 0.26 & 0.25 \\
\hline & & UB & 0.45 & 0.00 & 0.82 & 0.85 & 0.46 & 0.91 & 0.43 \\
\hline \multirow{3}{*}{$\begin{array}{l}\text { Accepting SNAP } \\
\text { benefits }\end{array}$} & \multicolumn{2}{|c|}{ Mean } & 0.46 & 0.33 & 0.71 & 0.78 & 0.33 & 0.56 & 0.51 \\
\hline & \multirow{2}{*}{\begin{tabular}{c|}
$95 \%$ \\
$\mathrm{CI}$
\end{tabular}} & LB & 0.26 & 0.02 & 0.44 & 0.44 & 0.02 & 0.15 & 0.40 \\
\hline & & UB & 0.67 & 0.65 & 0.98 & 1.12 & 0.65 & 0.96 & 0.62 \\
\hline \multirow{3}{*}{$\begin{array}{l}\text { Accepting WIC or } \\
\text { FMNP benefits }\end{array}$} & \multicolumn{2}{|c|}{ Mean } & 0.12 & 0.08 & 0.29 & 0.44 & 0.09 & 0.11 & 0.17 \\
\hline & \multirow{2}{*}{$\begin{array}{c}95 \% \\
\mathrm{CI}\end{array}$} & LB & -0.02 & -0.10 & 0.02 & 0.04 & -0.11 & -0.15 & 0.09 \\
\hline & & UB & 0.25 & 0.27 & 0.56 & 0.85 & 0.29 & 0.37 & 0.26 \\
\hline \multirow{3}{*}{$\begin{array}{l}\text { Matching programs for } \\
\text { SNAP benefits }\end{array}$} & \multicolumn{2}{|c|}{ Mean } & 0.35 & 0.00 & 0.29 & 0.22 & 0.17 & 0.33 & 0.24 \\
\hline & \multirow{2}{*}{$\begin{array}{c}95 \% \\
\text { CI }\end{array}$} & LB & 0.15 & 0.00 & 0.02 & -0.12 & -0.08 & -0.05 & 0.15 \\
\hline & & UB & 0.54 & 0.00 & 0.56 & 0.56 & 0.41 & 0.72 & 0.34 \\
\hline \multirow{3}{*}{$\begin{array}{l}\text { Nutrition or cooking } \\
\text { education }\end{array}$} & \multicolumn{2}{|c|}{ Mean } & 0.44 & 0.43 & 0.58 & 1.00 & 0.18 & 0.42 & 0.47 \\
\hline & \multirow{2}{*}{$\begin{array}{c}95 \% \\
\text { CI } \\
\end{array}$} & LB & 0.26 & 0.13 & 0.33 & 1.00 & -0.03 & 0.09 & 0.37 \\
\hline & & UB & 0.62 & 0.73 & 0.82 & 1.00 & 0.38 & 0.74 & 0.56 \\
\hline \multirow{3}{*}{ Health screenings } & \multicolumn{2}{|c|}{ Mean } & 0.03 & 0.00 & 0.11 & 0.22 & 0.00 & 0.08 & 0.06 \\
\hline & \multirow{2}{*}{$\begin{array}{c}95 \% \\
\mathrm{CI} \\
\end{array}$} & LB & -0.03 & 0.00 & -0.05 & -0.12 & 0.00 & -0.10 & 0.01 \\
\hline & & UB & 0.09 & 0.00 & 0.26 & 0.56 & 0.00 & 0.27 & 0.10 \\
\hline \multirow{3}{*}{$\begin{array}{l}\text { Transportation } \\
\text { services for consumers } \\
\text { to access operation }\end{array}$} & \multicolumn{2}{|c|}{ Mean } & 0.12 & 0.08 & 0.29 & 0.22 & 0.08 & 0.00 & 0.13 \\
\hline & $95 \%$ & LB & -0.02 & -0.10 & 0.02 & -0.12 & -0.10 & 0.00 & 0.06 \\
\hline & CI & UB & 0.25 & 0.27 & 0.56 & 0.56 & 0.27 & 0.00 & 0.21 \\
\hline & Me & & 0.38 & 0.25 & 0.50 & 0.44 & 0.00 & 0.22 & 0.32 \\
\hline $\begin{array}{c}\text { Operating a mobile } \\
\text { market }\end{array}$ & $95 \%$ & LB & 0.18 & -0.04 & 0.20 & 0.04 & 0.00 & -0.12 & 0.21 \\
\hline & CI & UB & 0.59 & 0.54 & 0.80 & 0.85 & 0.00 & 0.56 & 0.42 \\
\hline & Me & & 0.19 & 0.08 & 0.29 & 0.11 & 0.17 & 0.33 & 0.20 \\
\hline Subsidized farm shares & $95 \%$ & LB & 0.03 & -0.10 & 0.02 & -0.15 & -0.08 & -0.05 & 0.11 \\
\hline & CI & UB & 0.35 & 0.27 & 0.56 & 0.37 & 0.41 & 0.72 & 0.28 \\
\hline Education about & Me & & 0.84 & 0.93 & 0.74 & 1.00 & 0.47 & $\mathbf{0 . 8 3}$ & 0.79 \\
\hline community and food & $95 \%$ & LB & 0.71 & 0.77 & 0.52 & 1.00 & 0.21 & 0.59 & 0.71 \\
\hline systems issues & $\mathrm{CI}$ & UB & 0.98 & 1.08 & 0.95 & 1.00 & 0.74 & 1.08 & 0.87 \\
\hline Education programs in & Me & & 0.44 & 0.50 & $\mathbf{0 . 4 7}$ & 0.78 & 0.29 & 0.42 & 0.46 \\
\hline community or school & $95 \%$ & LB & 0.26 & 0.20 & 0.23 & 0.44 & 0.05 & 0.09 & 0.36 \\
\hline & CI & UB & 0.62 & 0.80 & 0.72 & 1.12 & 0.54 & 0.74 & 0.55 \\
\hline & Me & & 0.97 & 0.71 & 0.95 & 0.78 & 0.82 & 0.92 & 0.88 \\
\hline $\begin{array}{l}\text { Food donation to local } \\
\text { food pantries/banks }\end{array}$ & $95 \%$ & LB & 0.91 & 0.44 & 0.84 & 0.44 & 0.62 & 0.73 & 0.82 \\
\hline & CI & UB & 1.03 & 0.98 & 1.06 & 1.12 & 1.03 & 1.10 & 0.95 \\
\hline & Me & & 0.68 & 0.43 & 0.79 & 0.89 & 0.47 & 0.67 & 0.65 \\
\hline services or activities & $95 \%$ & LB & 0.50 & 0.13 & 0.59 & 0.63 & 0.21 & 0.35 & 0.55 \\
\hline & CI & UB & 0.85 & 0.73 & 0.99 & 1.15 & 0.74 & 0.98 & 0.74 \\
\hline Total Number of & Me & & 4.97 & 3.71 & 5.95 & 7.33 & 3.06 & 5.08 & 4.88 \\
\hline Community Services & $95 \%$ & LB & 4.14 & 2.80 & 4.48 & 5.49 & 1.79 & 3.45 & 4.36 \\
\hline Offered & CI & UB & 5.80 & 4.63 & 7.42 & 9.18 & 4.32 & 6.72 & 5.41 \\
\hline
\end{tabular}

Note: Species codes -1 = Average Joes; 2 = Small-scale Startups; 3 = Processors; 4= Community Service Providers; 5

= Coops; $6=$ Traditional Produce Warehousers. $\mathrm{CI}=$ confidence interval. $\mathrm{LB}=$ lower bound. $\mathrm{UB}=$ upper bound. 


\begin{abstract}
AUTHOR VITA
Jason Scott Entsminger is a native of Reno, Nevada, where he was born, grew up, and attended college at the state's land-grant institution - the University of Nevada, Reno. In his five years at the University of Nevada, he graduated with distinction in May 2010 with bachelor's degrees in agricultural and applied economics, international affairs - law, diplomacy, and comparative political systems, and French language and literature, along with minors in applied statistics and Italian studies. Throughout his undergraduate career with the Nevada Wolf Pack, Dr. Entsminger developed a record of accomplished service to both the university and the community. He was the Nevada FFA Association's State Sentinel in 2005 and National Officer Candidate in 2007, after which he led the association's state officer selection process for three years. He also served in the student government — the Associated Students of the University of Nevada — in highlevel positions, was an officer in a number of student organizations, and acted as an ambassador for the College of Agriculture, Biotechnology, and Natural Resources. Dr. Entsminger also worked as a research assistant in Nevada's Cooperative Extension Service and for the Northern Nevada International Center's Algerian Youth Leadership Program.

After his undergraduate career, he pursued a dual master's under the ATLANTIS Programme - an international cooperative agreement between the United States and the European Union. During the program, Dr. Entsminger completed a Master of Science in food and resource economics from the University of Florida and an International Masters of rural development from a consortium of institutions in the European Union. His studies in Europe included work at the Università degli studi di Pisa (Italy), the École Nationale Supérieure Agronomique de Rennes-Agrocampus Ouest (France), and
\end{abstract}


Humboldt-Universität zu Berlin (Germany). His thesis focused on assessing the effect of technical trade barriers - in the form of sanitary and phyto-sanitary regulations - by developed nations on the agricultural economies of their lesser-developed trading partners. Dr. Entsminger's work was recognized as the best thesis across all programs at the University of Florida's College of Agriculture and Life Sciences and his European degree was awarded with Greatest Distinction. Throughout the program, Dr. Entsminger also worked as a graduate research assistant at the University of Florida's Institute of Food and Agricultural Sciences, Florida's cooperative extension system. His work included teaching across the state in Annie's Project (a program for women entrepreneurs in agriculture), assisting with the launch of Florida MarketMaker, and supporting undergraduate students in a short-term study abroad program visiting France.

After defending his master's thesis in Berlin, Dr. Entsminger returned to the United States to begin his career in the Washington, DC, region. He crafted proposals for projects to support rural development in agricultural communities across the Global South with CNFA, a nonprofit organization focused on international agriculture. Then he was a lecturer in agricultural business management at the University of Maryland, where he taught courses in marketing, finance, management, and applied mathematics; supervised student internships and research projects; and coordinated the certificate program in agribusiness as academic advisor. At the University of Maryland, he decided to pursue a $\mathrm{PhD}$ and include research in his academic portfolio. The summer before beginning his doctoral program, Dr. Entsminger worked in the Pathways program at the US Department of Agriculture's Foreign Agriculture Service as an economist, supporting federal programs to promote the trade of US agricultural products internationally. This 
included program implementation with federal cooperators - industry organizations with special status - as as program monitoring and evaluation. He was recognized with a citation for meritorious service by the Deputy Administrator of the Foreign Agriculture Service for his work.

In August of 2015, Dr. Entsminger relocated to Columbia, Missouri, and began his doctoral studies in agricultural and applied economics at the University of Missouri. Entering Mizzou at a turbulent time in the institution's history, he quickly took on roles in student advocacy and governance, first through the social movement under the Forum of Graduate Rights, then under the union the Coalition of Graduate Workers, and then as part of the Graduate and Professional Council. He has also held service roles in the Division of Applied Social Sciences and the Graduate School, and as a reviewer for the Academy of Management, Agriculture and Human Values, and the Journal of Agriculture, Food Systems, and Community Development. Dr. Entsminger has spent the past five years working as a research associate at the McQuinn Center for Entrepreneurial Leadership. His portfolio includes work on organizational classification and evolution, identification-based models of member commitment to organizations, and the use of collective strategies by minority, marginalized, and socially disadvantaged entrepreneurs. This work is done through the lens of agrofood systems. He has also been responsible for teaching courses on the agricultural marketing system, strategic management, strategic entrepreneurship, and applied economics in the College of Agriculture, Food, and Natural Resources' Division of Applied Social Sciences and in the Trulaske School of Business. He has mentored a number of undergraduate and graduate students. Dr. Entsminger's contributions have been recognized through induction into the Rollins Society and the 
Mizzou 18, an award by the Graduate Professional Council as an outstanding member of the General Assembly, and by a Catalyst Award from the LGBTQ Resource Center. Having grown up in the mountains of the Sierra Nevada, Dr. Entsminger enjoys the beauty of public lands through hiking and kayaking. He is an avid traveler, which allows him to practice the six languages he speaks. As part of his outdoorsmanship and travel, Dr. Entsminger practices his primary creative endeavor: photography. He also refinishes antique furniture, which he began in an attempt to preserve heirlooms inherited from his great-grandmother- the first items she purchased after emigrating from Germany to the United States after World War II. Dr. Entsminger continues in active service to the LGBT+ community, which he has done for the past decade. That service has even included being an invited participant in the White House Convening on Advancing LGBT Progress in Rural America. He enjoys taking his English Pointer, Lizzie, to the dog park. He and his partner hope to found a heritage farm of their own where they will incorporate regenerative and traditional agricultural practices in a diversified, small-scale operation. 